\title{
Land-use impacts on biodiversity and ecosystem functioning of complex multitrophic communities
}

\author{
Dissertation \\ for the award of the degree \\ "Doctor of Philosophy" Ph.D. Division of Mathematics and Natural Sciences \\ of the Georg-August-Universität Göttingen \\ within the doctoral program Biology \\ of the Georg-August University School of Science (GAUSS)
}

submitted by

Andrew D. Barnes

from La Grande, Oregon

Göttingen, 2015 


\section{Thesis Committee}

Prof. Dr. Ulrich Brose, Systemic Conservation Biology, J.F. Blumenbach Institute of Zoology and Anthropology

Prof. Dr. Stefan Scheu, Animal Ecology, J.F. Blumenbach Institute of Zoology and Anthropology

\section{Members of the Examination Board}

Reviewer: Prof. Dr. Ulrich Brose, Systemic Conservation Biology, J.F. Blumenbach Institute of Zoology and Anthropology

Second Reviewer: Prof. Dr. Stefan Scheu, Animal Ecology, J.F. Blumenbach Institute of Zoology and Anthropology

\section{Further Members of the Examination Board:}

Prof. Dr. Nico Eisenhauer, Animal Ecology, J.F. Blumenbach Institute of Zoology and Anthropology

Prof. Dr. Holger Kreft; Biodiversity, Macroecology, and Conservation Biogeography;

Faculty of Forest Sciences and Forest Ecology

Prof. Dr. Mark Maraun, Animal Ecology, J.F. Blumenbach Institute of Zoology and Anthropology

Prof. Dr. Teja Tscharntke, Agroecology, Department of Crop Sciences 




\section{TABLE OF CONTENTS}

Summary

.IX

Author contributions

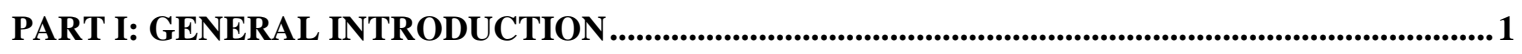

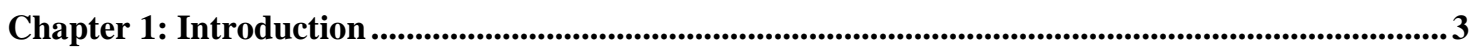

1.1 Understanding shifting ecosystems in a changing world ........................................................ 3

1.2 How land-use change impacts ecosystems ..................................................................... 4

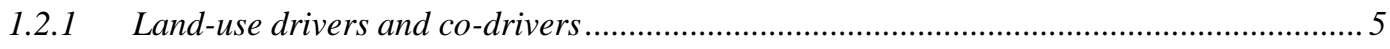

1.2.2 Diversity of ecological responses ............................................................................... 8

1.3 Land use in a connected world: the importance of species interactions ..................................9

1.4 The effect of changing biodiversity on ecosystem functioning ............................................. 12

1.4.1 Mechanisms underlying biodiversity-ecosystem functioning relationships ...................... 12

1.4.2 Biodiversity-ecosystem functioning relationships in real ecosystems............................. 15

1.5 Research objectives and chapter outline .............................................................................. 18

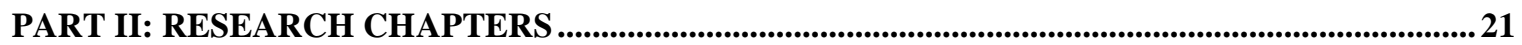

Chapter 2: Tropical Land-Use Intensification Imposes Direct and Cascading Impacts Across

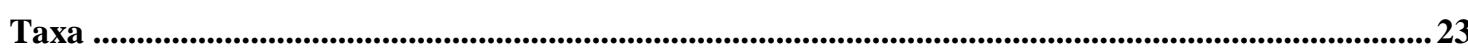

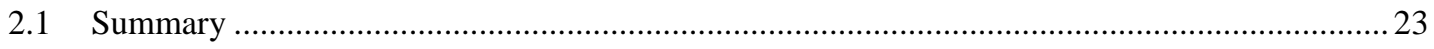

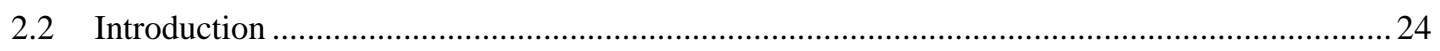

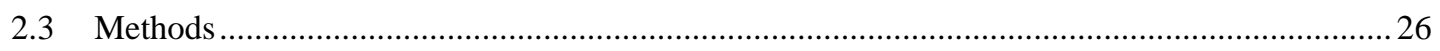

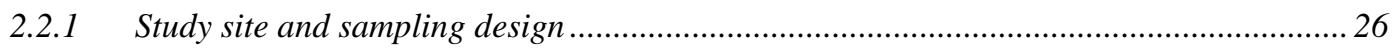

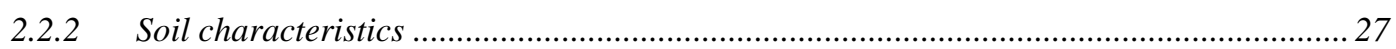

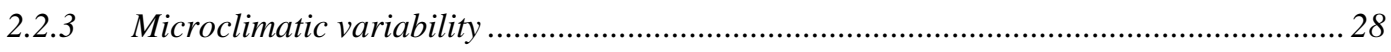

2.2.4 Plant diversity, above- and below-ground biomass .................................................... 29

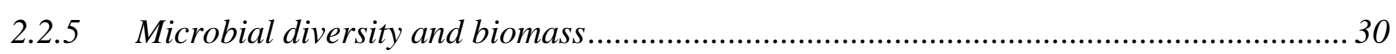

2.2.6 Litter macroinvertebrate diversity and biomass ........................................................ 31

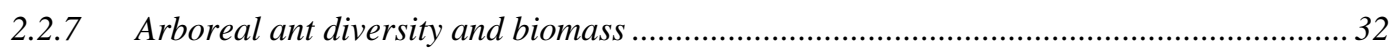

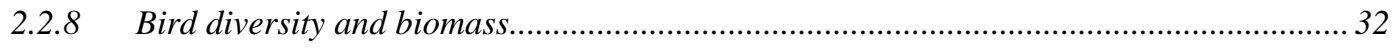

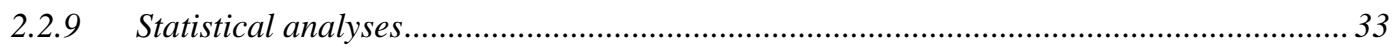

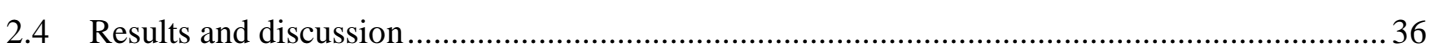

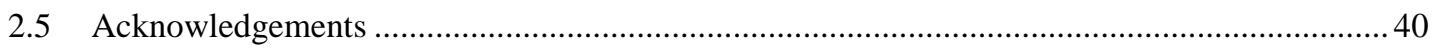

Chapter 3: Consequences of Tropical Land Use for Multitrophic Biodiversity and Ecosystem

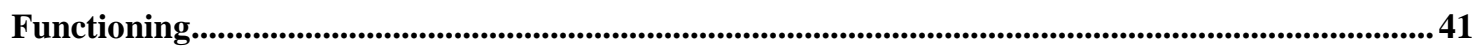

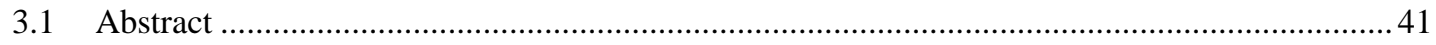

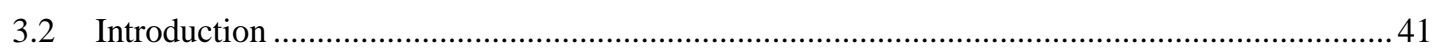

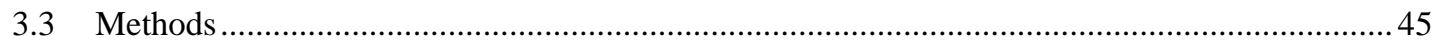

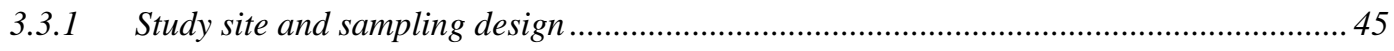


3.3.2 Animal sampling and calculation of response variables.................................................. 45

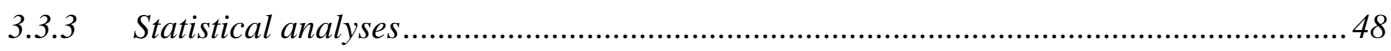

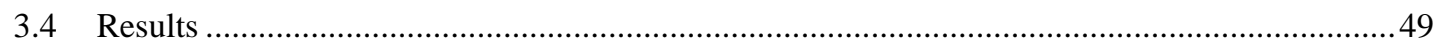

3.4.1 Transformation to oil-palm agriculture leads to biodiversity loss................................. 49

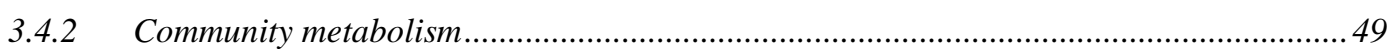

3.4.3 Whole-community energy fluxes and ecosystem functioning .........................................50

3.4.4 Multitrophic biodiversity-ecosystem function relationships ....................................... 52

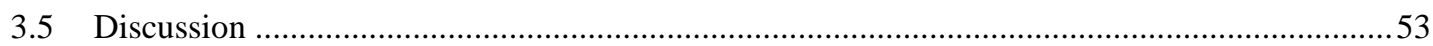

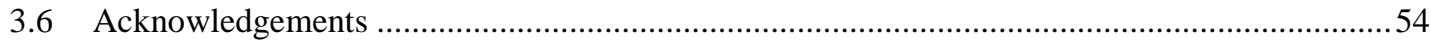

Chapter 4: Functional Diversity and Stability of Litter-Invertebrate Communities Following

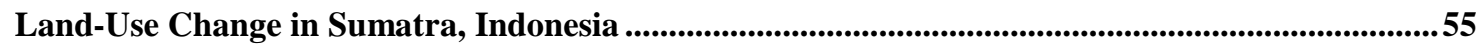

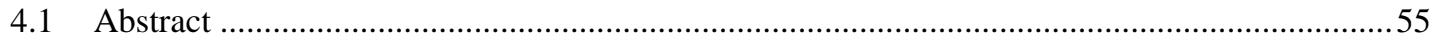

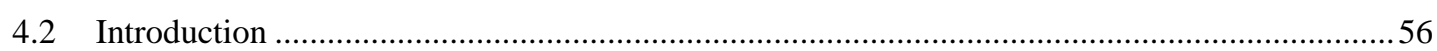

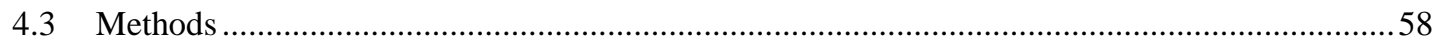

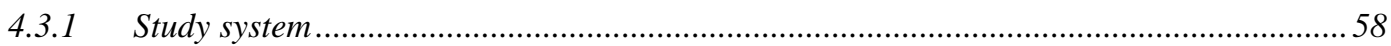

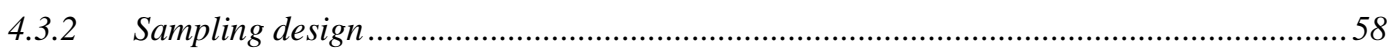

4.3.3 Animal identification and trait measurements .........................................................59

4.3.4 Functional group richness and functional dispersion..................................................... 61

4.3.5 Calculating measures of community stability and resilience ....................................... 62

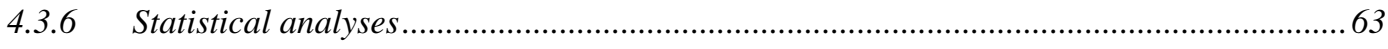

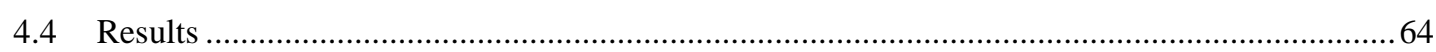

4.4.1 Species richness and animal densities ................................................................... 64

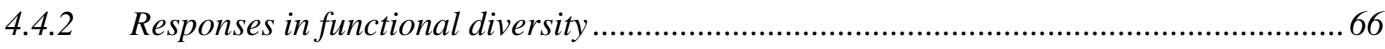

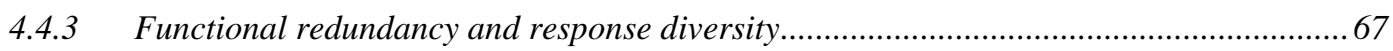

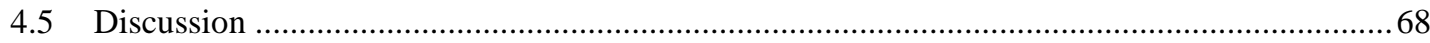

4.5.1 Effect of land-use change on species diversity and animal density..................................69

4.5.2 Land-use change alters functional diversity of litter macro-invertebrates ....................... 69

4.5.3 Functional redundancy and response diversity under land-use intensification................ 71

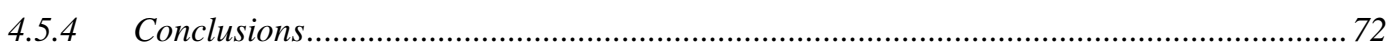

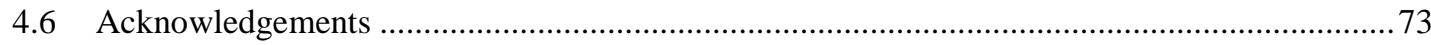

Chapter 5: Spatial Turnover in Biodiversity and Ecosystem Functioning Across Tropical and

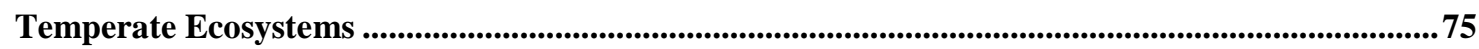

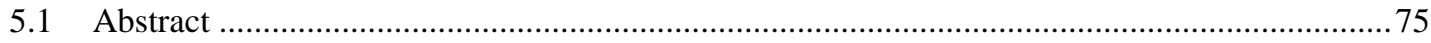

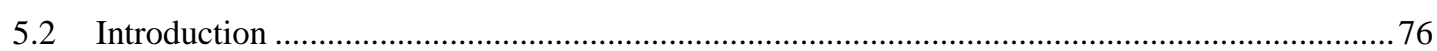

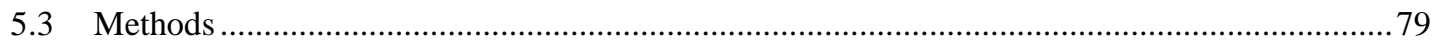

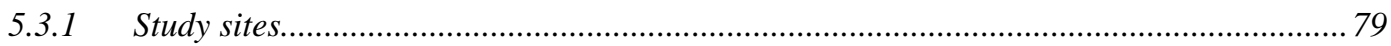

5.3.2 Quantifying spatial and environmental distance ............................................................ 79

5.3.3 Animal sampling and measurements..................................................................... 81

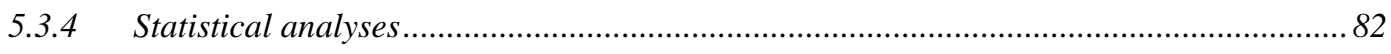

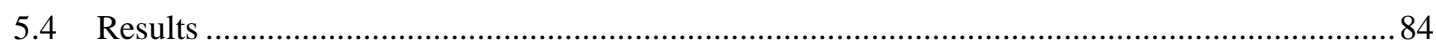




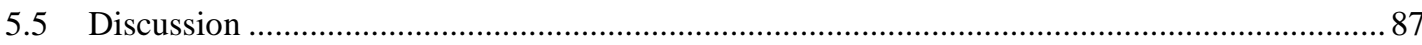

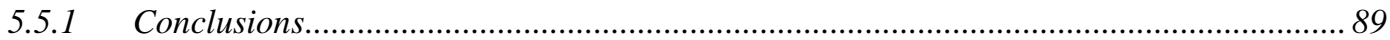

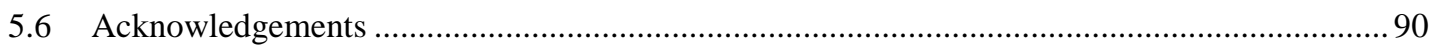

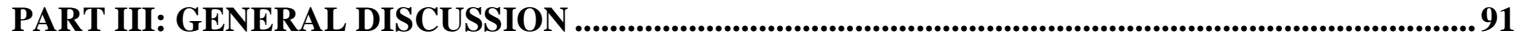

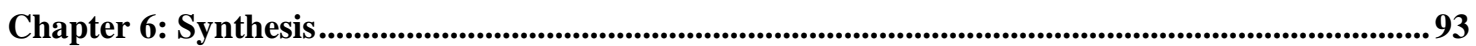

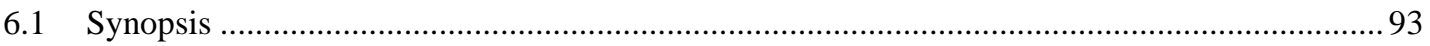

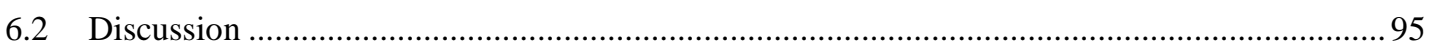

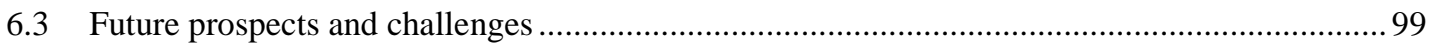

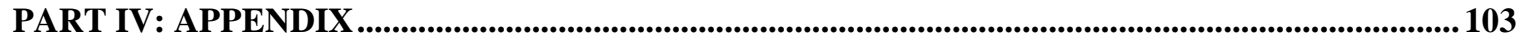

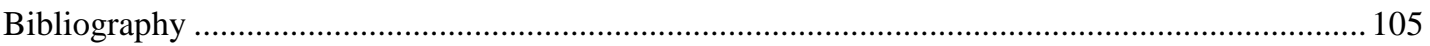

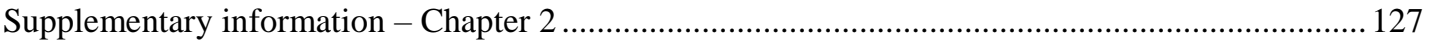

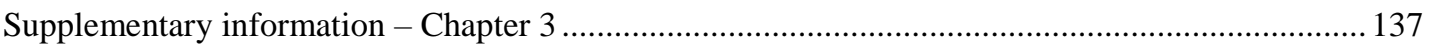

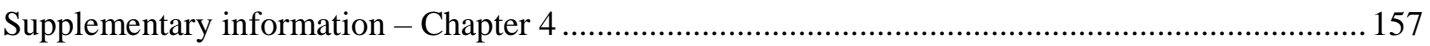

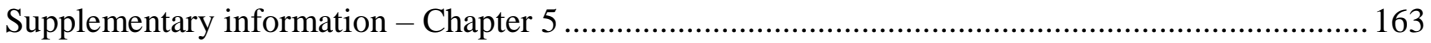

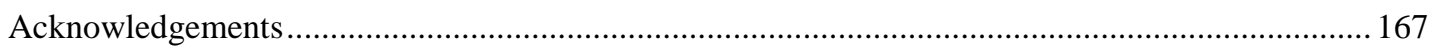




\section{Summary}

Global biodiversity is rapidly declining, resulting in far-reaching impacts on the functioning of ecosystems and human wellbeing. In recent decades, anthropogenic land use has been identified as a major driver of biodiversity loss, especially through the expansion and intensification of agricultural systems. While the drivers of biodiversity loss have been relatively clearly established, variability in the way that whole ecosystems respond to these drivers is still poorly understood. This is, in part, because we still lack a clear understanding of how species interactions govern the way that complex communities respond to environmental stressors, as well as their role in mediating ecosystem functioning.

Species interactions can moderate community responses to land-use change via trophic cascades, whereby extinctions at the top or bottom of a food chain produce cascading effects through the rest of the food web due to the disruption of resource availability or predatory control of consumers. Additionally, species interactions are fundamental for ecosystem functioning as they are almost always directly linked to processes such as decomposition, herbivory, predation, pollination, and seed dispersal. Therefore, an approach to studying biodiversity and ecosystem functioning of naturally complex communities that incorporates multiple trophic levels and their interactions is crucial for predicting future global-change scenarios. Despite the conceptual advantage of a multitrophic approach, this has been rarely applied in the context of biodiversity and ecosystem functioning of ecosystems undergoing land-use change. In addition, while there has been considerable evidence established for the role of biodiversity in maintaining ecosystem functioning in local-scale experiments, there is still very limited knowledge of how this relationship scales up to landscapes in real-world ecosystems. In this thesis, I aimed to achieve a conceptual advance in biodiversity-ecosystem functioning (BEF) research within the context of global environmental change by investigating responses of complex multitrophic communities to land-use change and the resulting consequences for ecosystem functioning.

Firstly, in Chapter 2, I combined data from a wide taxonomic range of trophic groups to test how communities of interacting species respond to tropical land-use intensification in Sumatra, Indonesia. I employed structural equation modelling to test if land-use intensification directly impacted all trophic groups or, alternatively, if it affected 
only lower trophic levels, resulting in bottom-up trophic cascades. Results from this model suggested that direct land-use impacts were generally much stronger than bottomup trophic effects. Interestingly though, the number of direct effects from land-use intensification decreased considerably from plants to predators, whereas the number of bottom-up trophic effects increased dramatically with increasing trophic level. These findings suggest that the underlying mechanisms of land-use intensification that alter communities highly depend on the trophic level in question, indicating the need for trophic level-specific conservation management strategies.

The results from Chapter 2 provided strong evidence for the importance of species interactions in moderating community responses to land use, leading to the question of how ecological processes carried out by multitrophic communities are resultantly affected. One major challenge of BEF research has been to fully incorporate species interactions across multiple trophic levels to quantify a trophically broad measure of ecosystem functioning. In Chapter 3, I overcame this challenge by developing a measure of ecosystem functioning that integrates food web and metabolic theory to calculate community energy flux across multiple trophic levels. By calculating energy flux of multitrophic macroinvertebrate communities, I demonstrated that declining species diversity with increasing land-use intensity led to concomitantly strong declines in community energy flux. Furthermore, I found that the relationship between species richness and energy flux was steeper in the most intense land-use system, oil palm, but this result did not hold when trophic guilds were analysed independently. Thus, these findings suggest that if trophic groups are omitted, it is possible that BEF relationships could be misinterpreted in response to anthropogenic land use.

In order to extend the previous chapter's findings beyond the provisioning of ecosystem functioning of multitrophic communities, in Chapter 4, I investigated the functional stability and resilience of the macroinvertebrate communities to future perturbations. Using a trait-based approach, I determined how communities were assembled among different land-use types. I then calculated functional stability and community resilience by measuring the number of functionally redundant species within functional effect groups (based on traits that determine species' influence on ecosystem processes) and the dispersion of traits within functional response groups (based on traits that determine species' responses to disturbances). In doing so, I found that litter invertebrate communities in oil palm plantations were more randomly assembled, as well as having significantly fewer functionally redundant species. However, the jungle rubber 
agroforest system harboured communities with considerably higher functional redundancy than in oil palm. These results indicate that communities in high-intensity land-use systems are more susceptible to functional collapse given future perturbations, but low-intensity agroforests could help to maintain higher functional stability in anthropogenic landscapes.

Finally, in Chapter 5, I investigated how ecosystem functioning varies across spatial and environmental gradients and the mechanisms that give rise to spatial turnover in ecosystem functioning. To test this, I used data on litter macroinvertebrate communities from landscapes in Indonesia and Germany and applied the energy flux calculations developed in Chapter 3 as a measure of multitrophic ecosystem functioning. I then employed structural equation modelling based on distance matrices to establish how environmental and geographic distance drive turnover in species composition, species richness, functional trait dispersion and community biomass, and how these factors consequentially drive spatial turnover in community energy flux in a tropical and temperate region. Environmental distance appeared to be more important in the Indonesian compared with the German region for driving species turnover. However, the mechanisms that determined spatial turnover in ecosystem functioning were remarkably similar between the tropical and temperate regions, such that species richness and community biomass were the most important variables explaining spatial variability in energy flux. These results suggest that mechanisms such as species identity and niche complementarity may become redundant for predicting ecosystem functioning at the landscape scale. Instead, species richness and biomass should be sufficient for predicting multitrophic ecosystem functioning at large spatial scales.

Overall, in this thesis I demonstrate that species interactions are important for mediating responses of multitrophic communities to land-use intensification and that the loss of species across trophic levels has drastic consequences for the provisioning of multitrophic ecosystem functioning. Furthermore, this species loss reduces the stability of ecosystem functioning in intensified agricultural landscapes. Finally, I demonstrate that species richness and community biomass are the key components for developing a framework aimed at predicting likely scenarios of functional losses in intensified land-use systems at the landscape scale. Ultimately, by incorporating real-world complexity into studies that integrate across multiple ecological concepts, this thesis presents a significant advance toward understanding how ecosystems respond to anthropogenic land-use change, thus highlighting important areas for future exploration. 


\section{Author contributions}

\section{Chapter 2: Tropical Land-Use Intensification Imposes Direct and Cascading}

Impacts Across Taxa

Andrew D. Barnes, Kara Allen, Marife D. Corre, Holger Kreft, Yann Clough, Malte Jochum, Edzo Veldkamp, Rolf Daniel, Kevin Darras, Lisa Denmead, Noor Farikhah Haneda, Dietrich Hertel, Alexander Knohl, Martyna M. Kotowska, Syahrul Kurniawan, Ana Meijide, Katja Rembold, Walesa Edho Prabowo, Dominik Schneider, Teja Tscharntke, and Ulrich Brose

A.D.B., K.A. and U.B. designed the study; A.D.B., K.A., M.J., K.D., L.D., M.M.K., S.K., A.M., K.R., W.E.P., and D.S. collected the data; A.D.B and K.A. analysed the data; A.D.B. and K.A. wrote the first draft of the manuscript with substantial further contributions from all other authors.

Chapter 3: Consequences of Tropical Land Use for Multitrophic Biodiversity and Ecosystem Functioning

Andrew D. Barnes, Malte Jochum, Steffen Mumme, Noor Farikhah Haneda, Achmad Farajallah, Tri Heru Widarto, and Ulrich Brose

A.D.B., M.J. and U.B. designed the study; A.D.B., M.J. and S.M. carried out the field and laboratory work; A.D.B. and M.J. prepared and analysed the data; all authors interpreted the results and wrote the paper.

\section{Chapter 4: Functional Diversity and Stability of Litter-Invertebrate Communities} Following Land-Use Change in Sumatra, Indonesia

Steffen Mumme, Malte Jochum, Ulrich Brose, Noor Farikhah Haneda, and Andrew D. Barnes

A.D.B., M.J. and S.M. designed the study; A.D.B., M.J. and S.M. carried out the field and laboratory work; A.D.B. and S.M. prepared and analysed the data; S.M. wrote the first draft of the manuscript with substantial further contributions from all other authors. 
Chapter 5: Spatial Turnover in Biodiversity and Ecosystem Functioning Across Tropical and Temperate Ecosystems

Andrew D. Barnes, Patrick Weigelt, Malte Jochum, David Ott, Dorothee Hodapp, Noor Farikhah Haneda, and Ulrich Brose

A.D.B., P.W. and U.B. conceived and designed the study; A.D.B., M.J. and D.O.

collected the data; A.D.B. and P.W. analysed the data; A.D.B. wrote the first draft and all authors contributed substantially to the writing of the final manuscript. 


\section{Part I}

General Introduction 
Chapter 1

\section{Introduction}

\subsection{UNDERSTANDING SHIFTING ECOSYSTEMS IN A CHANGING WORLD}

The earth's ecosystems are currently shifting in an unprecedented manner; a process which is primarily dominated by human influence (Zalasiewicz et al. 2011). We are witnessing the greatest rate of species extinctions since the end of the CretaceousPaleogene transition (Barnosky et al. 2011; Ceballos et al. 2015). As well as dramatic losses of species, the earth is undergoing extraordinary levels of global biotic exchange (Chen et al. 2011; van Kleunen et al. 2015), giving rise to novel species interactions and vast shifts in the functioning of ecosystems (Tylianakis et al. 2008a). Understanding and mitigating the consequences of such enormous changes in the earth's biological systems has become one of the greatest challenges that humanity presently faces (Ehrlich \& Ehrlich 2013). To take on such challenges, specific aspects of global change and the ensuing biological responses must be elucidated. Firstly (but not exclusively), the major drivers of ecological shifts must be identified. Specifically, we must consider the relative impacts of different global change drivers such as anthropogenic land use, climate change, and nitrogen deposition and how they may interact to alter biological systems.

Secondly, an understanding of how different ecosystems and their ecological constituents (e.g., populations, species, food webs, and meta-communities) respond to these aforementioned global change drivers is crucial for predicting future biological outcomes in our changing world. This requires an understanding of how ecological communities, including species interactions and their traits, are structured across space and time. Thirdly, we must determine how these changes in the structure of biological systems alter the way that these ecological constituents perform by understanding the links between ecological structure and ecological processes. While these aspects of biological responses to global change drivers have all received rapidly increasing attention in recent years, we are still lacking a clear and fully comprehensive synthesis of scientific understanding. 
In this thesis, my aim is to achieve a major advance in our understanding of how complex biological systems will likely respond to one of the most important global change drivers; anthropogenic land use. Focusing primarily on shifting tropical agricultural landscapes in Indonesia, I investigate the impacts of different land-use types on ecological communities comprising a wide range of taxa and trophic levels. To ascertain how complex ecological systems respond to land-use change, I develop two overarching hypotheses of how taxonomically broad, multitrophic communities will be impacted by agricultural intensification — either by direct land-use effects, or indirectly via bottom-up trophic interactions - and disentangle these hypotheses within a structural equation modelling framework (Chapter 2). Then, to determine how multitrophic biodiversity responses to land-use intensification result in alteration of ecosystem functioning, I develop a multitrophic measure of ecosystem functioning, i.e. community energy flux, and analyse the consequences of biodiversity loss for multitrophic litter macro-invertebrate communities (Chapter 3). I further investigate the consequences of land-use change on the future certainty of the provisioning of ecosystem functions by using traits to estimate the functional resilience and stability of these litter macroinvertebrate communities (Chapter 4). Finally, I widen the geographic scope to include tropical and temperate litter invertebrate communities and investigate the aspects of community structure that drive spatial variation in multitrophic ecosystem functioning (Chapter 5). In doing so, this thesis presents a comprehensive advance in our understanding of how biological systems, in all their complexity, will likely be altered in response to anthropogenic land-use change and the consequences these alterations will yield for ecosystem functioning.

\subsection{HOW LAND-USE CHANGE IMPACTS ECOSYSTEMS}

Fifteen years ago, Sala and colleagues (2000) predicted the impacts of various drivers of ecological change on the earth's biomes. Using expert knowledge, the authors ranked major drivers of change based on expected impacts on global biodiversity in the year 2100, suggesting that anthropogenic land use would be the chief culprit for driving biodiversity loss in terrestrial ecosystems. Despite an element of subjectivity in this study, we are now seeing these global projections of biodiversity loss take shape (Newbold et al. 2015). As the human population grows at an increasingly rapid rate, maintaining the 
security of natural capital becomes ever more challenging (Ehrlich, Kareiva \& Daily 2012). This has led to widespread deforestation resulting from agricultural expansion, particularly in developing countries (Lambin \& Meyfroidt 2011; Laurance, Sayer \& Cassman 2014), as well as to increasing agricultural intensification (Matson et al. 1997). Specifically, in the developed world there has been considerable effort to increase crop yields. Nevertheless, these increases in intensification are believed to be insufficient to meet growing human demands (Gibbs et al. 2010). This has prompted a more recent expansion of agricultural land in the tropics where large tracts of unused arable land still exist, but this expansion frequently occurs at the expense of tropical rainforests (Gibbs et al. 2010) that harbour a high proportion of the earth's biodiversity (Myers et al. 2000; Dirzo \& Raven 2003). Consequently, deforestation and the conversion of natural ecosystems to agricultural production landscapes in the tropics has become a prominent concern for the conservation of global biodiversity (Gardner et al. 2009; Gibson et al. 2011).

Although most concern has been focused on the loss of biodiversity following land-use change, there exist a myriad of other ecological responses to land use. Such variability in ecological responses is partly due to differences in types of land use and interacting drivers of change (e.g., climate change or nitrogen deposition), but also because of variability in the biotic and abiotic attributes of ecosystems at different temporal and spatial scales (DeFries, Foley \& Asner 2004; Ewers \& Didham 2006; Gardner et al. 2009; Tscharntke et al. 2012b). In order to grasp the variety of ways that land-use change can impact ecosystems, we must consider the different aspects of landuse change that alter species assemblages and their influences on ecosystem processes. The factors that give rise to variation in responses of biological systems to land use can be conceptually divided into two major categories: 1) variation in land-use drivers and codrivers and 2) diversity of ecological responses to these drivers (DeFries et al. 2004). To discuss these factors, I will focus on agricultural expansion and intensification, although there are many other forms of anthropogenic land use such as mining of precious materials, urbanisation, and power generation that I will not cover here.

\subsubsection{Land-use drivers and co-drivers}

Land-use-related drivers of ecological change typically occur in two important phases. Initially, natural or unmodified landscapes are transformed to anthropogenic production landscapes. Following this landscape conversion, these production landscapes 
can then undergo varying practices that either increase or decrease the intensification of land use (Lambin, Geist \& Lepers 2003; DeFries et al. 2004; Lambin \& Meyfroidt 2010). During the initial 'transformation phase', natural ecosystems undergo habitat loss and fragmentation (DeFries et al. 2004); both of which can have contrasting, yet comparably important impacts on ecosystems (Fahrig 2003; Tscharntke et al. 2012b).

Habitat loss is perhaps the most noticeable driver of ecological change resulting from anthropogenic land use as it often occurs as an abrupt conversion of forest to croplands (DeFries et al. 2004). This process of conversion generally results in a severe reduction of habitat area and complexity through the removal of structurally complex plant communities. Perhaps unsurprisingly, this loss of habitat as a result of deforestation has severe impacts on resident species assemblages across a large range of taxa (Gibson et al. 2011; Pimm et al. 2014). Habitat loss through conversion to agricultural landscapes can even have indirect impacts on ecosystems, such as through the facilitation of invasive species in modified landscapes (Didham et al. 2007). At present, some of the most concerning cases of deforestation for species conservation are in the tropics (Gibbs et al. 2010; Laurance et al. 2014). In particular, Southeast Asia is undergoing an unprecedented rate of deforestation for the establishment of agricultural crops such as oil palm, rubber, and fibre plantations (Li et al. 2007; Fitzherbert et al. 2008; Koh et al. 2011; Wilcove et al. 2013; Abood et al. 2014). As a result of this land transformation, there have been increasing reports of large-scale species losses from many different taxonomic groups, especially within the Southeast Asian archipelago (Gibson et al. 2011).

With the conversion of natural habitat into agricultural landscapes, this can also result in the fragmentation of remnant ecosystems. While habitat loss and fragmentation tend to occur simultaneously, they have contrasting effects on ecosystems (Fahrig 2003; Tscharntke et al. 2012b). Fragmentation of habitats per se typically results in an increase of patch isolation and edge effects (Kupfer, Malanson \& Franklin 2006). In landscapes where habitat patches are more isolated, species that are dependent on these habitat patches tend to suffer from declines in genetic diversity due to an inability of individuals to disperse and outbreed to other populations in the wider landscape (Corlatti, Hackländer \& Frey-Roos 2009). The isolation of habitats is also likely to interact synergistically with global climate change by reducing the ability of species to track changing climates across fragmented landscapes (Hof et al. 2011). Edge effects generally increase in fragmented landscapes due to an increase in the edge-to-area ratio of remnant habitat patches. These effects have widely been shown to negatively impact ecosystems, probably due to a range 
of factors such as increases in microclimatic variability, wind disturbance, solar radiation, alteration of vegetative structure and increased predation pressure (Laurance et al. 1998; Vetter, Rücker \& Storch 2013; Didham \& Ewers 2014). The impacts that edge effects have on organisms, however, differ widely among taxa and are also dependent on the type of agricultural matrix present (Campbell et al. 2011; Barnes et al. 2014a). This is because anthropogenic landscapes are generally mosaics of different land uses that are subjected to various levels of management intensities, thus presenting a range of habitat types for species inhabiting these mosaic landscapes (Kupfer et al. 2006; Fahrig et al. 2011). As a result, anthropogenic land use can have distinctly contrasting ecological impacts at both the landscape and the local scale (Batáry et al. 2011; Gonthier et al. 2014).

Of the many different local management practices, material inputs such as fertiliser and pesticide application present some of the most acute effects in agricultural ecosystems (Matson et al. 1997; Tilman et al. 2002). The negative impacts of pesticide usage have been shown to outweigh impacts of other management practices on invertebrates in European croplands (Geiger et al. 2010), with evidence also mounting for detrimental effects on non-target microbial communities (e.g., Schuster \& Schröder 1990; Zhang et al. 2009). It is becoming increasingly recognised that pesticides are used in greater quantities and are also being more frequently overused in the tropics, which will almost certainly have severe consequences for biodiversity in tropical agricultural ecosystems (Wanger, Rauf \& Schwarze 2010). While the intention of pesticide application is to control pest species and prevent loss of crops, often their use results in negative effects on non-target species that may be beneficial for crop productivity, such as on species that provide natural biological control (Isaacs et al. 2009).

Following conversion of forest to agricultural production land, there is typically a significant loss of soil carbon and nitrogen from these systems (Murty et al. 2002). In more intensive agricultural systems, however, this is generally followed by very high inputs of nitrogen and phosphorus through the application of synthetic fertilisers (Tilman et al. 2002). Fertiliser inputs have been shown to have especially conspicuous negative effects on plant diversity by increasing competitive exclusion among species (Hautier, Niklaus \& Hector 2009). At larger scales, heavier fertiliser application has even been shown to have detrimental effects on bird species richness (Billeter et al. 2008), although this effect is probably indirect via the negative impacts of fertilisation on plant species richness. In addition to material inputs, the initial reduction and active clearance of understory vegetation and leaf litter also negatively impacts many different inhabitant 
taxa in agricultural systems (Lawton et al. 1998). For example, Bestelmeyer \& Wiens (1996) demonstrated that the structure of litter-dwelling ant communities was strongly affected by the amount of leaf litter covering the ground, which was altered as a result of varying land-use practices. Also, the clearance of understory vegetation (typically carried out in plantation agriculture systems) is likely to be responsible for declines in species richness such as those seen following the conversion of rainforest to oil palm plantations (e.g., Koh \& Wilcove 2008; Turner \& Foster 2008). In spite of these findings, direct experimental evidence for the importance of reduced understory vegetation on animal communities is still limited.

\subsubsection{Diversity of ecological responses}

The ways in which ecological communities are affected by land-use change are not only dependent on the type or intensity of land use, but also on the variability in responses of species to these aforementioned drivers (Tscharntke et al. 2012b). This largely arises from intra- and interspecific variability in traits of species that confer differing abilities to cope with environmental stressors. While there are indeed an infinite number of traits that vary continuously among species and individuals (Violle et al. 2012), only certain traits are variable enough to have an important influence on the way that an organism performs and responds to environmental stressors. These traits are commonly referred to as 'functional traits' (Violle et al. 2007). Species responses to habitat fragmentation have been repeatedly shown to be trait-dependent. Traits that mediate such responses often include dispersal ability and trophic generalism (Larsen, Lopera \& Forsyth 2008; Öckinger et al. 2010), quite possibly because higher dispersal ability allows organisms to move more freely among isolated patches, whereas higher generalism allows for the use of variable resources in the matrix habitat, thus reducing the deleterious effects of patch isolation. Additionally, trophic level has been shown to be an important factor determining species' sensitivities to habitat loss, such as in a study by Cagnolo et al. (2009) who found that parasitoids were more strongly affected than their leaf-miner prey and plants, suggesting the importance of bottom-up trophic cascades in response to land-use change. In addition to the initial loss and fragmentation of habitat, intensification of land use also imposes trait-dependent impacts on species. For example, a recent study by Rader et al. (2014) showed that, from small home gardens to large-scale intensive croplands, large-bodied, solitary, specialist pollinators were the most affected by intensified land use. Similar trait-mediated responses to land-use intensification have 
been found for plant communities, such as in the study by Laliberté \& Tylianakis (2012), which demonstrated that specific leaf area mediated responses in plant annual primary productivity to increasing nutrient inputs over 27 years of land-use intensification.

Thus far, I have predominantly focused on how specific traits can mediate individual and species responses to land-use change. Yet, functional traits can also play an important role in how whole communities respond to environmental stressors imposed by land use. In particular, the functional diversity of communities (i.e., the diversity of functional traits in a community) can have an important influence on how communities are assembled (Mouchet et al. 2010; Pakeman 2011) and how resilient these species assemblages are to land-use change (Laliberté et al. 2010). Because land-use change is a complex and dynamic process that comprises a wide range of environmental stressors (as discussed above), it therefore stands to reason that species assemblages composed of a wider range of traits will be better equipped to deal with such a multitude of stressors (Elmqvist et al. 2003; Folke et al. 2004; Mori, Furukawa \& Sasaki 2013). There is now rapidly increasing evidence that land-use change has detrimental impacts on the resilience of ecosystems due to the loss of functional diversity in communities of plants (Laliberte $e t$ al. 2010) and birds (Fischer et al. 2007; Karp et al. 2011). Such evidence is still lacking, however, for other functionally important taxonomic groups such as invertebrates and microbes. Nevertheless, these pioneering studies show that following land-use change, the diversity of functional traits that confer different responses to environmental stress in plant and bird communities is reduced. As a result, the ability of communities to maintain the provisioning of ecosystem functions given likely future disturbances—such as climate change and increased biotic exchange-will be at risk.

\subsection{LAND USE IN A CONNECTED WORLD: THE IMPORTANCE OF SPECIES INTERACTIONS}

"What escapes the eye is a much more insidious kind of extinction: the extinction of ecological interactions.” Daniel H. Janzen (1974)

There is a staggering amount of evidence for the detrimental impacts of anthropogenic land use on population abundances, species diversity, functional trait diversity, and community composition. Given such widespread impacts on populations and 
communities, this raises another important question: how do land-use impacts alter species interactions and what consequences do these altered interactions have for biodiversity and ecosystem functioning? In a seminal review of previous research into responses of species interactions to global-change drivers, Tylianakis et al. (2008) elucidated how the ecological impacts of global change will strongly depend on the way that species interact with each other. To understand how ecosystem responses can so strongly depend on species interactions, one must first understand how ecological processes can be altered by such interactions. Although the ways in which species can interact are highly numerous and complex (Wootton 1994), I will focus mostly on trophic interactions, but also briefly touch on mutualistic and competitive species interactions, with examples of how they might alter ecosystem responses to land-use change.

Trophic interactions have long been a focus in ecology as a means of understanding how resources are used and energy is transferred among organisms (Paine 1980). Because of the vital reliance of organisms on their resources, as well as the conversely strong pressure of predation on resource populations, these so called 'bottomup' and 'top-down' forces can instigate trophic cascades following perturbations to lower or higher trophic levels (Wootton 1994). For example, Scherber et al. (2010) showed that in experimental grassland communities, plants were highly important for structuring communities across a large range of taxa occupying multiple trophic positions because of bottom-up trophic interactions. This suggests that if these grasslands were subjected to perturbations that alter the plant communities (such as high levels of fertilisation typical of intensive agricultural systems [Tilman et al. 2002; Hautier et al. 2009]), the higher trophic levels in these systems would also be strongly affected due to their trophic dependencies on the diversity and biomass of their resources. Such disturbance-related, bottom-up trophic cascades have been experimentally shown in other simple two- or three-trophic-level systems (Haddad et al. 2009; Borer et al. 2012). However, any clear test of these indirect bottom-up cascading effects in ecosystems undergoing land-use change is still lacking (Gardner et al. 2009). Nevertheless, there appears to be strong evidence that bottom-up cascades can have important implications for ecosystem functioning and the provisioning of ecosystem services. For example, Ebeling et al. (2014) found that higher plant diversity resulted in significantly higher rates of litter decomposition and herbivory, suggesting that these processes could be altered in agriculturally intensified landscapes where plant diversity is reduced. 
While bottom-up forces are clearly important for structuring multitrophic ecosystems, they do not act in isolation from top-down forces (Hunter, Varley \& Gradwell 1997; Denno et al. 2003). Through the removal of top predators, top-down forces can also drive dramatic shifts in ecosystems (Pace et al. 1999). For example, a marine study of a four-trophic-level system in the eastern Scotian Shelf found that overfishing of cod resulted in cascading effects on lower trophic levels, releasing shrimp and crap populations from predation and thus diminishing planktonic biomass (Frank et al. 2005). The instigation of trophic cascades in terrestrial ecosystems as a result of landuse change is also likely to be highly important, but evidence for these processes is still lacking and this topic will require considerably more attention with increasing land-use intensification (Gardner et al. 2009).

The conversion of natural to anthropogenically-dominated landscapes not only has direct impacts on locally inhabitant species, but also increases the likelihood of species spillover from neighbouring habitats (Rand, Tylianakis \& Tscharntke 2006; Frost et al. 2014), as well as facilitating the expansion of invasive species (Didham et al. 2007). This exchange of species can result in the emergence of novel interactions in altered ecosystems, such as competition or predation, that further impact the structure and functioning of communities (Tylianakis et al. 2008a). Aside from the mediating role of species interactions on land-use impacts in multitrophic communities, land-use change can also have important effects on species interactions that directly alter ecosystem functioning (Chapin III et al. 2000). This has been shown in a Europe-wide study where the long-term use of pesticides resulted in major declines in predatory invertebrates, consequently reducing biocontrol of aphids in crop systems (Geiger et al. 2010). Pollination mutualisms are perhaps one of the most economically important species interactions because of their crucial role in the production of food crops, yet invertebrate pollinators are currently facing global declines (Tylianakis 2013). These declines are related to an array of driving factors, although anthropogenic land-use-particularly due to pesticide application and agricultural intensification - is most likely one of the major drivers of these declines (Kremen et al. 2007; Potts et al. 2010). In general, because of the multitrophic impacts of land-use change on complex biological systems that are interconnected by species interactions (Tylianakis et al. 2008a), the way that resources are transferred throughout these systems will be strongly affected. Essentially, this generalisation points to the fact that taking into account the multitrophic structure of 
ecological systems will be crucial for determining how land-use change will alter biodiversity and related ecosystem functioning (Dobson et al. 2006; Reiss et al. 2009).

\subsection{THE EFFECT OF CHANGING BIODIVERSITY ON ECOSYSTEM FUNCTIONING}

Nearly a decade ago, Vitousek et al. (1997) proposed that the earth has been virtually dominated by anthropogenic activities, resulting in the global alteration of important ecosystem processes such as $\mathrm{CO}_{2}$ sequestration and $\mathrm{N}$ fixation. Their review invoked the concept that anthropogenic activities do not only impact ecosystems locally but also at a global scale, thus highlighting the serious concern over the potential loss of ecosystem services (i.e., ecosystem functions that are beneficial to human wellbeing). Land-use change, in particular, threatens the provisioning of ecosystem services; for example, through increases in carbon dioxide and nitrous oxide emissions, increased disease transmission (DeFries et al. 2004), and reduced food security (Tscharntke et al. 2012a). The growing body of evidence that human-induced global change alters ecosystem processes and threatens human wellbeing has spurred on an intensive effort to understand how ecosystem functions are lost in altered ecosystems (Cardinale et al. 2012). In the 1980s, concern over increasing rates of biodiversity loss prompted investigation of how organisms alter and regulate ecosystem processes, leading to a general cognisance that anthropogenically-driven species loss could severely compromise the provisioning of ecosystem functioning (Cardinale et al. 2012). By the 1990s, these conceptual advances had developed into a new field of ecological research aimed at understanding the relationship between biodiversity and ecosystem functioning (BEF), thus marking the advent of the BEF paradigm (Tilman, Isbell \& Cowles 2014).

\subsubsection{Mechanisms underlying biodiversity-ecosystem functioning relationships}

By the turn of the century, a vast number of experimental studies had managed to clearly identify the likely mechanisms underlying BEF relationships (Hooper et al. 2005). These studies indicated that the ways in which biodiversity can alter functioning could be divided into two major categories: how biodiversity regulates 1) stocks and fluxes of energy, and 2) stability of ecosystem functions (Srivastava \& Vellend 2005). These broad categories can then be further broken down into the specific mechanisms that regulate such phenomena. Regarding stocks and fluxes of energy, I will focus on two well- 
established general mechanisms; niche complementarity and identity effects (Hooper et al. 2005). I will then briefly discuss the importance of biodiversity for the stability of ecosystem functions.

Niche complementarity occurs when an increase in the number of species allows for the partitioning of resources among these species, thus reducing competition and allowing for greater resource assimilation of a given trophic level. This phenomenon was shown to be a likely explanation for BEF relationships in a modelling study by Tilman et al. (1997), where additional plant species resulted in greater community biomass production when there were two resources available and competition was permitted. This phenomenon is also known as 'overyielding', whereby primary production in multispecies systems yields greater biomass than would be expected in an average monoculture (Hooper et al. 2005). While the theoretical basis for niche complementarity as an explanatory mechanism underlying BEF relationships is well established, this mechanism has been rather challenging to demonstrate in experimental studies. However, a laboratory study by Striebel et al. (2009) provided an excellent example of niche complementarity in phytoplankton communities. Essentially, they found that with increasing species richness of phytoplankton there was an increase in biomass. This is because different phytoplankton species absorb different wavelengths of light, meaning that in more diverse communities there is an overall increase in light absorption as these different species are able to exploit different niches throughout the light spectrum, thus increasing overall primary productivity. A more recent study by Hoehn et al. (2008) used an agricultural field experiment to test for spatial niche complementarity in pollinator organisms. In this study, they found that more pollinator functional groups led to greater overall pollination rates of pumpkin flowers because different species of pollinators tend to fly at different heights. As a result, higher pollinator functional diversity led to greater exploitation of the flowers that were distributed across a range of heights, demonstrating complementary use of resources within pollinator communities. Both of these studies clearly suggest that niche complementarity relies on trait differences within species assemblages, but also importantly depends on resource heterogeneity, which allows for the partitioning of different resources within trophic levels (Tylianakis et al. 2008b). Therefore, niche complementarity might be less often observed in simpler systems with fewer species and less resource niches.

In contrast to niche complementarity, identity effects can occur without necessarily observing changes in species richness. Essentially, identity effects refer to 
disproportionate effects of particular species on ecosystem functioning, whereby the inclusion or removal of such species in a community will result in disproportionate gains or losses in rates of functioning (Hooper et al. 2005). The importance of identity effects first started receiving considerable attention when it was realised that biodiversity effects found in some early BEF experiments were probably a result of the confounded selection of competitively dominant and highly productive species (Huston 1997). More specifically, it was discovered that in these BEF experiments where diversity was manipulated - either through the random selection of species from a larger species pool, or through the removal of species by inducing competitive exclusion through fertiliser application (e.g., Naeem et al. 1994; Tilman, Wedin \& Knops 1996) — there was an increased probability of selecting competitively dominant and/or highly productive species, resulting in higher overall biomass production. Therefore, this experimental phenomenon has been called the 'sampling effect' or the 'selection probability effect' (Hooper et al. 2005; Srivastava \& Vellend 2005). Interestingly, these experimental mishaps shed new light on the likely importance of species differences and community composition, generating a new line of investigation into how species' identities alter BEF relationships. One such early study demonstrated that, while species richness was still positively correlated with biomass production in experimental plant communities, the BEF relationship was highly dependent on the presence of leguminous species (Symstad et al. 1998). Identity effects have since been suggested to hold great importance for the functional stability of ecosystems in cases where there might be positive covariance between the vulnerability of species and their functional importance. For example, Larsen, Williams \& Kremen (2005) demonstrated that larger-bodied dung beetles and pollinating bees are more functionally important (due to higher dung removal and pollinating capabilities compared to smaller-bodied species), but are also more prone to anthropogenic disturbances. Therefore, they suggested that ecosystems may be at risk of rapid collapses in ecosystem functioning due to non-random loss of the most functionally important species following anthropogenic disturbances.

Aside from demonstrating that biodiversity could increase the assimilation of resources and production of biomass, there is also strong evidence for the positive effect of biodiversity on the stability of ecosystem functioning (Tilman et al. 2014). The idea that diversity begets stability is not a recent one, and was most notably proliferated by Charles Elton (1958) who suggested that more speciose communities were less prone to invasion by exotic species. In BEF research, species richness has been suggested to 
positively affect the stability of ecosystem functioning through two major mechanisms: the redundancy of functionally similar species and the temporal asynchrony of functionally similar species (Hooper et al. 2005; Srivastava \& Vellend 2005). The insurance hypothesis proposes that with increasing species richness of functionally similar species (species within the same trophic level or functional group), the provisioning of ecosystem functioning should be more robust to ecological perturbations because of the redundancy of these functionally similar species. For example, Yachi \& Loreau (1999) demonstrated that more species led to greater stability of biomass production in model communities subjected to a fluctuating environment. This occurred because if certain species reduced their productivity, others were able to take their place and maintain constant overall ecosystem productivity. Additionally, in ecosystems where rare species are functionally redundant (i.e., many rare species are present that carry out similar ecosystem functions), this would allow for the loss of more species before such a shared ecosystem function would entirely collapse (Naeem \& Wright 2003). This phenomenon has been demonstrated in South American plant communities where it was shown that in high diversity communities, $75 \%$ of the species could potentially go extinct before entire functional groups were lost (Fonseca \& Ganade 2001). The asynchrony of species can also promote functional stability through the so-called 'portfolio effect', whereby more speciose communities have more differently fluctuating populations. As such, when certain species are reduced in abundance, it is more likely that other species will have higher abundances and thus maintain total ecosystem productivity in highdiversity communities (Isbell, Polley \& Wilsey 2009). In contrast to the insurance hypothesis, the portfolio effect confers temporal stability of the provisioning of ecosystem functioning, such as the maintenance of primary productivity, through the asynchrony of species' abundances.

\subsubsection{Biodiversity-ecosystem functioning relationships in real ecosystems}

Since the beginnings of BEF research in the 1990s, there has been a proliferation of studies that both support and dispute the causal relationship between biodiversity and ecosystem functioning (Balvanera et al. 2006). In particular, there has been general concern that the experimental studies which gave rise to much of the theoretical foundations of BEF research provide little application to the real world (Duffy 2009). However, in the past decade there have been considerable advancements and important 
conceptual shifts in the way that BEF research is carried out in order to better incorporate ecological realism into the development of BEF theory (Reiss et al. 2009).

One such advancement has been the shift toward incorporating species traits into BEF experiments. Originally, identity effects were seen as a mechanism that drives idiosyncratic responses of ecosystem functioning to species diversity (Emmerson et al. 2001) and have even been suggested as a departure from BEF questions (Bengtsson 1998). On the contrary, if taxonomic identities are translated into trait identities that can be measured on a continuous scale, such trait-based approaches to BEF research can be very powerful and can help to explain seemingly idiosyncratic BEF relationships (Reiss et al. 2009). In particular, the use of functional diversity (rather than taxonomic diversity) has been suggested as a strong predictor of ecosystem functioning (Díaz \& Cabido 2001; Flynn et al. 2011). This is because functional diversity is a description of biodiversity based on functionally important characteristics of organisms, thus providing a better compositional representation of communities and taking into account species identities as well as total number of species (Petchey \& Gaston 2006). In a recent investigation of the relationship between functional traits and ecosystem functioning, Gagic and colleagues (2015) found that functional diversity and community trait means were much better predictors of ecosystem functioning than species diversity across a range of invertebrate taxa and their associated ecosystem functions. Another recent study furthered these ideas by experimentally manipulating functional diversity of multitrophic estuarine communities, showing that multiple ecosystem functions were better explained by functional diversity than taxonomic diversity and that increased functional diversity gave rise to higher rates of functioning via niche complementarity and identity effects (Lefcheck \& Duffy 2015). In addition to demonstrating the importance of trait variability for BEF research, Lefcheck \& Duffy (2015) also highlight the growing awareness for the importance of taking into account multiple ecosystem functions that arise from multitrophic systems in BEF studies.

As discussed previously, different species are distributed over many different trophic levels and thus assimilate different resources in their environment. As a result, in real-world ecosystems there is a vast diversity of ecosystem functions carried out by different organisms at different trophic levels (Cardinale et al. 2006). From these concepts, there has recently been a strong development of the idea that biodiversity can predict 'multifunctionality', or the provisioning of multiple ecological functions (Reiss et al. 2009). For example, Hector \& Bagchi (2007) presented a compelling analysis of the 
effects of species diversity on multifunctionality, showing that with increasing species richness there is a general increase in the number of different ecosystem functions carried out in a given system. These findings were further extended to show that as more ecosystem functions are considered, the importance of biodiversity for ecosystem multifunctionality increases, suggesting that previous studies focusing on individual functions may have grossly underestimated the role of biodiversity on ecosystem functioning (Lefcheck et al. 2015). One issue with the concept of ecosystem multifunctionality has been the development of indices that can be used to encompass a wide range of ecosystem functions. Until recently, studies investigating multifunctionality have used contrasting measures that often do not provide a clear indication of the value of ecosystem functioning (Byrnes et al. 2014). While an ecosystem multifunctionality approach is a promising direction for taking into account multiple functions in BEF research, this approach is still in its infancy and has yet to be clearly developed into a widely applicable method for assessing biodiversity effects on ecosystem functioning.

Another less explored avenue of BEF research is the use of species interactions to quantify ecosystem functioning. Essentially, most ecosystem functions such as carbon production, nutrient uptake, and denitrification, are properties that emerge from the interactions of organisms with their resources or other species (Chapin III et al. 2000; Reiss et al. 2009). Early work by de Ruiter et al. (1993, 1994) introduced a highly promising way of modelling nutrient cycling based on the trophic interactions of soil food webs. Specifically, they suggested that the feeding rate (and thus material uptake) of soil organisms is not only dependent on their biomasses but also on their efficiency of resource uptake (how much energy is assimilated versus what is excreted) as well as the natural and predation-dependent death rate of consumers. As such, this calculation of feeding rates to estimate multitrophic energy flow and nutrient cycling provided a measure of ecosystem functioning that is highly dependent on food-web structure. Therefore, it presents a major step toward the incorporation of interaction network structure into BEF research. Since this ground-breaking work, considerable attention has been given to the importance of $\mathrm{BEF}$ research within the context of network theory (Duffy et al. 2007). It is now more clearly recognised that trophic complexity, as found in real-world ecosystems, is important for regulating the way that biodiversity is related to ecosystem functioning (Duffy et al. 2007). Trophic cascades resulting from anthropogenic disturbances provide direct examples of how trophic interactions can greatly alter rates of ecosystem functioning (Pace et al. 1999). In addition to increasing 
trophic realism in BEF research, studies have begun to consider the importance of different spatial scales (Cardinale et al. 2012), despite the difficulty in assessing BEF relationships at the level of landscapes (Gagic et al. 2015). If we are to begin to understand how the mechanisms underlying BEF relationships that have been identified in experimental studies scale up to larger spatial scales, such as the landscape or regional level, BEF research must be applied to such scales. For example, Chalcraft et al. (2004) found that the relationship between species richness and plant productivity shifted from a null or weak hump-shaped relationship at the local level to a strong hump-shaped relationship at the regional level. This result indicates that the findings derived from the plethora of small-scale, experimental BEF studies may contradict those from larger-scale studies. Certainly, one of the greatest challenges and, indeed, most exciting avenues currently on the horizon for BEF research is the incorporation of trophic complexity and simultaneous quantification of multiple ecosystem functions at large spatial scales in complex, real-world ecosystems (Reiss et al. 2009).

\subsection{RESEARCH OBJECTIVES AND CHAPTER OUTLINE}

In the research chapters of this thesis, I address how land-use change alters taxonomically diverse multitrophic communities and how this leads to changes in the rates and stability of ecosystem functioning. I then delve into the underlying mechanisms that determine spatial variation in rates of multitrophic ecosystem functioning. Overall, this thesis aims to present an advancement in our understanding of how species interactions mediate the impacts of land-use change on ecosystems and how variation in biodiversity of multitrophic communities is responsible for real-world variation in ecosystem functioning carried out by trophically complex communities. In order to investigate these objectives, I predominantly utilise data from the large-scale collaborative research initiative, EFForTS (CRC990), within the subproject entitled "Structure, stability and functioning of macroinvertebrate communities in rainforest transformation systems in Sumatra (Indonesia)", comparing macroinvertebrate communities among tropical agricultural land-use systems of varying intensities (from rainforest to monoculture plantation agriculture). In Chapter 5, I also draw on data from within a large collaborative research project in Germany, the Biodiversity Exploratories, in order to compare biodiversityecosystem functioning patterns between tropical and temperate ecosystems. 
To establish the role of species interactions in mediating the impacts of land-use change on multitrophic systems, In Chapter 2 I investigate whether land-use change imposes predominantly direct effects across trophic levels or if the effects are indirect via bottom-up trophic cascades. Firstly, I develop two distinct hypotheses of how land-use change impacts multitrophic systems: 1) the direct land-use effects hypothesis, and 2) the cascading land-use effects hypothesis. To test these hypotheses, I gather biomass and species richness data from various subprojects within the EFForTS project including plants, soil microbes, litter macroinvertebrates, arboreal ants, and birds that span a total of five trophic levels, and also gather abiotic data including soil and microclimatic parameters. Then, using structural equation modelling, I test for the presence of bottomup trophic impacts versus direct effects of land use on these different taxa and trophic levels. From this taxonomically and trophically broad structural equation model, I evaluate the relative importance of direct versus bottom-up cascading effects at different trophic levels. In summary, by combining a multitrophic food web perspective with the use of structural equation modelling, I effectively disentangle the way in which complex ecosystems respond to tropical land-use change.

While Chapter 2 aims to shed light on the importance of species interactions for mediating changes in biomass and biodiversity of multitrophic systems subjected to landuse change, it raises the question of what consequences such alterations to multitrophic communities have for ecosystem functioning. In Chapter 3, I investigate how land-use change alters biodiversity of litter macroinvertebrate communities and how the functioning of these communities is altered as a result. Because these litter macroinvertebrate communities encompass a range of taxa that belong to different trophic levels, I use a combination of food-web theory and metabolic theory to develop a singular measure of multitrophic ecosystem functioning, energy flux, that can be analytically calculated for sampled communities. I then relate changes in species richness with wholecommunity energy fluxes among land-use systems of varying intensities to determine whether biodiversity-ecosystem functioning relationships are dependent on the land-use context, as well as looking for evidence of functional redundancy in these multitrophic systems.

In addition to how land-use change alters rates of multitrophic ecosystem functioning, I set out to investigate how vulnerable litter macroinvertebrate communities in these tropical land-use systems are to the collapse of entire ecosystem functions. Therefore, in Chapter 4, I test for the impacts of land-use change on the functional 
stability and resilience of litter macroinvertebrate communities in order to understand how likely these communities are to lose whole ecosystem functions. To test this, I use measured functional traits including body size, mobility, feeding type, and eusociality to calculate functional diversity. Furthermore, I divide the macroinvertebrate communities into distinct functional groups based on the importance of these traits for species responses to environmental stressors (response traits) as well as their importance for influencing ecosystem processes (effect traits). I then calculate the level of trait variability within each functional response group (response diversity) as a measure of functional resilience, as well as the number of species within each functional effect group (functional redundancy) as a measure of functional stability. Finally, I test for differences in response diversity and functional redundancy among different land-use types in order to ascertain whether the functional resilience and stability of these communities is reduced with increasing land-use intensification.

Building on the establishment of how land-use change alters multitrophic communities via species interactions and the consequences of these effects for the provisioning and stability of multitrophic ecosystem functioning, in Chapter 5 I aim to determine the underlying mechanisms that give rise to variation in rates of ecosystem functioning. Specifically, I posit that rates of energy flux in multitrophic communities are likely to vary significantly through space and also across environmental gradients. Furthermore, I identify four major hypotheses derived from BEF theory that propose how spatial turnover in community attributes will drive spatial variation in ecosystem functioning: 1) the species identity hypothesis, 2) the species richness hypothesis, 3) the niche complementarity hypothesis, and 4) the total biomass hypothesis. To test these hypotheses in concert, I use a structural equation modelling framework based on distance matrices to determine how spatial and environmental distance drive turnover in four associated community attributes: species composition, species richness, functional diversity, and biomass. In doing so, this chapter identifies the mechanisms that mediate rates of ecosystem functioning in multitrophic communities through space. 


\section{Part II}

\section{Research Chapters}


Chapter 2

\title{
Tropical Land-Use Intensification Imposes Direct and Cascading Impacts Across Taxa
}

\author{
Andrew D. Barnes, Kara Allen, Marife D. Corre, Holger Kreft, Yann Clough, Malte \\ Jochum, Edzo Veldkamp, Rolf Daniel, Kevin Darras, Lisa Denmead, Noor Farikhah \\ Haneda, Dietrich Hertel, Alexander Knohl, Martyna M. Kotowska, Syahrul Kurniawan, \\ Ana Meijide, Katja Rembold, Walesa Edho Prabowo, Dominik Schneider, Teja \\ Tscharntke, and Ulrich Brose
}

\subsection{SUMMARY}

Land-use change is driving rapid declines in global biodiversity, with the most drastic foreseen consequences in the tropics (Newbold et al. 2015). The conversion of tropical forests to rubber and oil-palm monocultures (Gibbs et al. 2010; Koh et al. 2011; Wilcove et al. 2013) impact a large range of taxa across whole food webs (Tylianakis et al. 2008a), with severe consequences for biodiversity and ecosystem functioning (Foster $e t$ al. 2011). It remains unknown, however, if these responses are driven by direct land-use effects or by bottom-up trophic cascades (Kagata \& Ohgushi 2006; Gardner et al. 2009). Combining data from a multi-taxa research initiative in Indonesia, we test for direct landuse effects (i.e., that directly affect each trophic level) versus cascading land-use effects (i.e., mediated by bottom-up trophic interactions) across multitrophic communities. Here, we show that $90 \%$ of land-use impacts on plants and belowground taxa at lower trophic levels are direct, with $67 \%$ of these effects driving severe declines in biomass and diversity. In contrast, $70 \%$ of all land-use impacts at the highest trophic levels of invertebrates and birds are bottom-up controlled. We demonstrate that the impacts of tropical land-use intensification alternate from direct to cascading trophic effects with increasing trophic level, collectively driving large-scale ecological shifts. Clear strategies for preventing ecological collapses in human-altered tropical landscapes will rely on the incorporation of species interactions to inform conservation management at different trophic levels. 


\subsection{INTRODUCTION}

Anthropogenic activities have altered most of the earth's ecosystems (Vitousek et al. 1997), largely through the expansion and intensification of agricultural landscapes for food production and biofuels (Foley et al. 2005; Laurance et al. 2014). In tropical regions, which harbour exceptionally high levels of biodiversity (Connell 1978; Basset et al. 2012), the conversion of rainforest to monoculture cash crops, such as oil palm and rubber, is currently the greatest threat to global biodiversity (Li et al. 2007; Fitzherbert et al. 2008; Wilcove et al. 2013; Laurance et al. 2014). This threat will almost certainly worsen with rising global demands for food, fuel and fibre (Koh \& Ghazoul 2008). Recent studies have shown that these monoculture systems are associated with reduced biodiversity across multiple trophic levels (Foster et al. 2011), leading to impaired ecosystem functioning (Allen et al. 2015; Kotowska et al. 2015). Temperate and tropical research has long focused on how human land use directly affects one or two trophic groups (e.g., plants, birds or butterflies), without considering indirect effects via bottomup forces. In recent years, though, more attention has been turned toward quantifying land-use impacts on multitrophic systems (Tylianakis et al. 2008a). These studies effectively demonstrate that multitrophic interactions underlie the provisioning of ecosystem functioning and that neglecting these interactions may lead to biased inference on the extent of land-use consequences. Until now, however, research has been predominantly restricted to few trophic levels (Tylianakis et al. 2008a), without causal tests of cascading effects through multiple trophic levels across many higher-level taxa (Gardner et al. 2009; Tscharntke et al. 2012b). This research gap creates uncertainty about whether land-use impacts propagate upward through multiple trophic levels to the top of food chains directly (Figure 2.1a) or indirectly via trophic cascades (Figure 2.1b).

In order to grasp the scale of ecological change in altered tropical landscapes and to understand the mechanisms driving these changes, a multi-taxa whole-ecosystem approach is needed. In recent years, experimental research that manipulates plant diversity and composition has elucidated how primary producers influence the structure of food webs via bottom-up trophic cascades (Denno et al. 2002; Scherber et al. 2010; Narwani \& Mazumder 2012; Borer et al. 2012; Ebeling et al. 2014), demonstrating that organisms at lower trophic levels are key to understanding how whole ecosystems are structured. This notion has crucial implications for the way that land-use intensification will impact ecosystems and how such impacts will be most effectively assessed and 
mitigated. If theory suggests that the alteration of plant diversity and biomass should impose strong bottom-up trophic cascades (Haddad et al. 2009; Scherber et al. 2010; Borer et al. 2012), these trophic cascades may be more important than the direct impacts of management practices in intensified land-use systems. On the one hand, direct land-use effects could dominate through cases such as pesticide application imposing direct toxic effects on invertebrates and microbes, or regular disturbance of nesting birds in intensively managed plantations (Figure 2.1a). In contrast, cascading effects could prevail for heterotrophic consumers that may be strongly impacted by the depletion of their resources; an effect that could propagate from altered plant communities (Figure 2.1b). A clear understanding of these processes is critical for discerning the drivers of biodiversity decline and functional loss in anthropogenic landscapes, as well as being essential for making informed conservation management decisions across trophic levels.
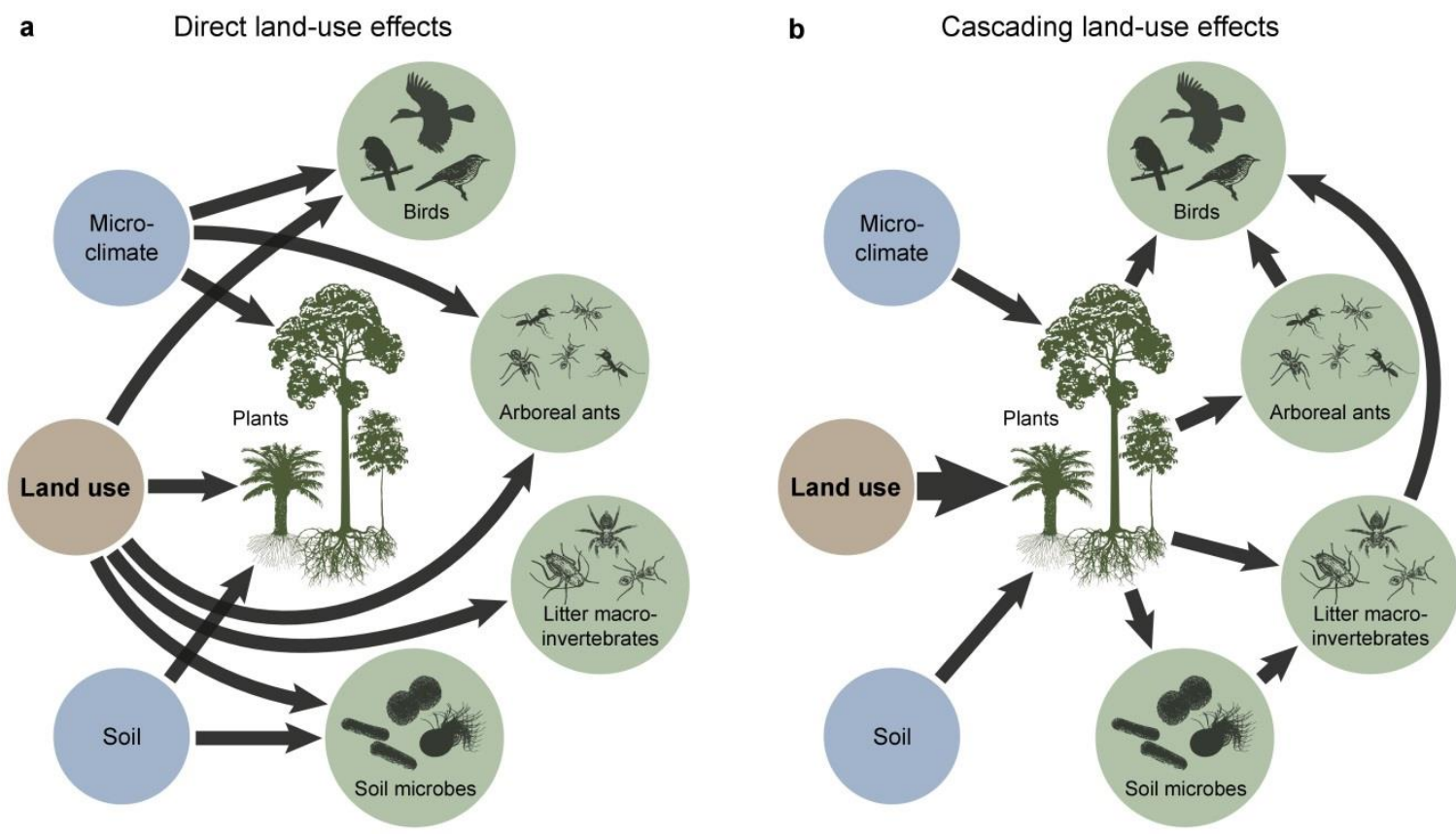

Figure 2.1: Two alternative hypotheses for how land-use change will alter whole ecosystems. The direct land-use effects hypothesis (a) assumes land-use intensification will directly impact all taxonomic groups, irrespective of any bottom-up trophic effects. The cascading land-use effects hypothesis (b) assumes that all impacts of land-use intensification on biota at higher trophic levels will propagate via bottom-up processes. Effects are grouped into land-use effects (brown), abiotic effects (blue), and biotic effects (green).

Here, for the first time, we develop and test distinct hypotheses for the direct (Figure 2.1a) versus cascading (Figure 2.1b) effects of anthropogenic land use, employing a structural equation modelling framework to construct broad-scale food webs that 
include taxa ranging from microbes to birds. Using data from an extensive multi-taxa research initiative, we sampled species richness (hereafter, diversity) and biomass of

plants, bacteria, archaea, litter invertebrates, arboreal ants, and birds (Supplementary Table 2.1) in lowland forest, jungle rubber, rubber monocultures, and oil palm monocultures in Sumatra, Indonesia (Supplementary Figure 2.1). Additionally, we created composite variables of 'soil organic matter', 'nutrient availability', and microclimatic variability (hereafter, 'microclimate') from soil properties and variability in atmospheric and soil microclimate using principal components analysis (PCA). All variables were standardised by subtracting their means and dividing by twice their standard deviations to allow direct comparisons of effect sizes (Gelman 2008). To test for direct (Figure 2.1a) versus indirect cascading land-use effects (Figure 2.1b), we constructed generalised multilevel path models (a form of structural equation modelling) (Shipley 2009), allowing for direct and interactive effects of land use with abiotic variables and only allowing for biologically relevant bottom-up trophic effects among biotic variables (Supplementary Figure 2.2).

\subsection{METHODS}

\subsubsection{Study site and sampling design}

The study was carried out in Jambi Province, Sumatra, Indonesia. Study sites were located within two landscapes in Jambi Province: the Harapan landscape $\left(1^{\circ} 55^{\prime} 40^{\prime}\right.$ S, $103^{\circ} 15^{\prime} 33^{\prime \prime}$ E, elevation of $70 \pm 4 \mathrm{~m}$ above sea level [asl]) and the Bukit Duabelas landscape $\left(2^{\circ} 0\right.$ ' $57^{\prime}$ ' S, $102^{\circ} 45^{\prime} 12^{\prime \prime} \mathrm{E}$, at an elevation of $75 \pm 4 \mathrm{~m}$ asl) (Supplementary Figure 2.1). The mean annual temperature is $26.7 \pm 1.0^{\circ} \mathrm{C}$ and mean annual precipitation is $2235 \pm 385 \mathrm{~mm}$ (1991-2011; climate station at the Jambi Sultan Thaha airport of the Indonesian Meteorological, Climatological and Geophysical Agency). Natural vegetation in the region is composed of mixed Dipterocarp forests (Kotowska et al. 2015). The soils in the two landscapes are classified as Acrisol soils, which comprises $49.9 \%$ of the land area in Sumatra and are the dominant soil type in the study area (FAO et al.). The soil texture is the main difference between the two landscapes in terms of soil physical characteristics, with predominantly clay Acrisol soils found in Bukit Duabelas and loam Acrisol soils found in Harapan (Allen et al. 2015). 
Four land-use systems common to the region were examined: primary degraded forest (Margono et al. 2014), jungle rubber, rubber monoculture, and oil palm monoculture. In each landscape, four $50 \mathrm{~m}$ x $50 \mathrm{~m}$ replicate plots per land-use type were selected with a minimum distance of $200 \mathrm{~m}$ between plots $(\mathrm{n}=32$; Supplementary Figure 2.1). A $10 \mathrm{~m} \times 10 \mathrm{~m}$ grid was established across each replicate plot, and five randomly selected $5 \mathrm{~m}$ x $5 \mathrm{~m}$ subplots were established within each plot, where the majority of the sampling activities took place. The primary degraded forest sites were located within two protected areas, Bukit Duabelas National Park and the Harapan Rainforest, and represent the least influenced system. Jungle rubber (extensively managed rubber agroforest with a high density of native trees) represented a low-impact agroforestry system (Gouyon, Foresta \& Levang 1993), while the rubber and oil palm plantations served as intensivelymanaged monocultures. Intensity of management practices varied between the rubber and oil palm systems. Soil amendments, such as fertilization and liming, only occurred in the oil palm plantations either once or twice per year, dependent on smallholder preference. Both chemical and manual weeding took place throughout the year in rubber and oil palm plantations. Labour was more intensive in the rubber plantations, which required manual tapping on average three days per week, while harvesting of oil palm fruits occurred biweekly (Euler et al. unpublished data).

\subsubsection{Soil characteristics}

Soil sampling was conducted between June 2013 and December 2013. Soil samples for soil biochemical analysis were collected from ten randomly selected points located at least $5 \mathrm{~m}$ distance from the plot's border within each $50 \mathrm{~m}$ x $50 \mathrm{~m}$ replicate plot. Soil samples were taken from the top $0.1 \mathrm{~m}$ soil depth, within an area of $0.4 \mathrm{~m} \times 0.4$ $\mathrm{m}$ at each point. The soil had no organic layer but only a thin litter layer, and this was removed in order to sample predominantly mineral soil. Soil biochemical characteristics for each replicate plot were represented by the average of the ten individual subplots. Soil samples were air dried and sieved $(2 \mathrm{~mm})$ at the University of Jambi, Indonesia and sent to the Soil Science of Tropical and Subtropical Ecosystems (SSTSE) laboratory at GeorgAugust University Göttingen, Germany for analysis. Soil $\mathrm{pH}\left(\mathrm{H}_{2} \mathrm{O}\right)$ was analysed in a 1:4 soil-to-water ratio. Soil organic $\mathrm{C}\left(\mathrm{SOC} ; \mathrm{kg} \mathrm{C} \mathrm{m}^{-2}\right)$ and total $\mathrm{N}\left(\mathrm{g} \mathrm{N} \mathrm{m}^{-2}\right)$ concentrations were analysed from air-dried, sieved $(2 \mathrm{~mm})$ and ground samples using a $\mathrm{CN}$ analyser (Vario EL Cube, Elementar Analysis Systems GmbH, Hanau, Germany). Air-dried and sieved soils were used to determine effective cation exchange capacity $\left(\mathrm{ECEC} ; \mathrm{mmol}_{\mathrm{c}} \mathrm{kg}^{-}\right.$ 
${ }^{1}$ ) by percolating with unbuffered $1 \mathrm{~mol} \mathrm{~L}^{-1} \mathrm{NH}_{4} \mathrm{Cl}$, and cations (Ca, $\mathrm{Mg}, \mathrm{K}, \mathrm{Na}, \mathrm{Al}, \mathrm{Fe}$, and $\mathrm{Mn}$ ) were measured in percolates using an inductively coupled plasma-atomic emission spectrometer (iCAP 6300 Duo VIEW ICP Spectrometer, Thermo Fischer Scientific GmbH, Dreieich, Germany). Base saturation was calculated as percent exchangeable base cations of the ECEC. Extractable $\mathrm{P}\left(\mathrm{g} \mathrm{P} \mathrm{m}^{-2}\right)$ was determined using the Bray 2 method, which is used for acidic tropical soils (Bray \& Kurtz 1945). For ${ }^{15} \mathrm{~N}$ natural abundance signatures $\left(\delta^{15} \mathrm{~N} ;{ }^{0} \%\right.$ ) , the ten subsamples from each replicate plot were composited, ground and analysed using isotope ratio mass spectrometry (IRMS; Delta Plus, Finnigan MAT, Bremen, Germany).

Soil texture (\% sand, \% silt and \% clay) was measured using the pipette method (Gee \& Bauder 1986) from soil cores taken in the top $0.1 \mathrm{~m}$ soil depth from soil profiles dug at three of the four replicate plots per land-use type $(n=24)$. The core method was used to measure soil bulk density for the top $0.5 \mathrm{~m}$ soil depth from two of the randomly assigned subplots (Blake \& Hartge 1986). Soil bulk density $\left(\mathrm{g} \mathrm{cm}^{-3}\right)$ for each replicate plot was represented by the mean of the two individual subplots. Soil moisture (\%) was measured using moisture sensors (IMKO Trime-PICO, Ettlingen, Germany) recorded every hour with a data logger (see Microclimatic variability). Soil sampling was conducted using the collection permits 2703/IPH.1/KS.02/XI/2012 and S.13/KKH-2/2013 recommended by the Indonesian Institute of Sciences (LIPI) and issued by the Ministry of Forestry (PHKA).

\subsubsection{Microclimatic variability}

Weather stations were installed in the centre of each of the 32 replicate plots. They were equipped with thermohygrometers (Galltec Mella, Bondorf, Germany) placed at a height of $2 \mathrm{~m}$ to record air temperature $\left({ }^{\circ} \mathrm{C}\right)$ and humidity $(\%)$ inside the canopy, and soil sensors (IMKO Trime-PICO, Ettlingen, Germany) at $0.3 \mathrm{~m}$ depth, to monitor soil temperature $\left({ }^{\circ} \mathrm{C}\right)$ and moisture $(\%)$. Both sensors were connected to a data logger (LogTrans16-GPRS, UIT, Dresden, Germany) and measured every hour. The $5^{\text {th }}$ and $95^{\text {th }}$ percentiles, from data available for the period June 2013 to October 2014, for air temperature, humidity and soil temperature were used to calculate ranges for each variable. Climate data were collected using the research permits 389/FRP/SM/II/2013 and 05/EXP/SIP/FRP/SM/II/2014 recommended by the Indonesian Institute of Sciences (LIPI). 


\subsubsection{Plant diversity, above- and below-ground biomass}

In each replicate plot, all trees with a diameter at breast height $(\mathrm{DBH}) \geq 10 \mathrm{~cm}$ were identified and measured (spatial position within the plot, height, DBH, crown structure). All vascular plant individuals growing within the five subplots were identified and measured (height). Whenever possible, herbarium specimens were prepared from three individuals per species for identification and were later archived at several Indonesian herbaria (Herbarium Bogoriense, BIOTROP Herbarium, UNJA Herbarium, Harapan Rainforest Herbarium). All individuals measured on the plots were identified to morphospecies based on consistent morphological characteristics. Vascular plant sampling was conducted using the collection permit 2266/IT3/PL/2013 recommended by the Indonesian Institute of Sciences (LIPI) and issued by the Ministry of Forestry (PHKA). Plant diversity was calculated as total sampled species richness within each replicate plot.

Within each replicate plot, all trees, palms and lianas with $\mathrm{DBH}>10 \mathrm{~cm}(\mathrm{DBH}$ at $1.3 \mathrm{~m}$ ) were tagged. The DBH was measured using measuring tape (Richter Measuring Tools, Speichersdorf, Germany) and total height was recorded using a Vertex III height meter (Haglöf, Långsels, Sweden). Wood density values (dry mass per fresh volume in kg $\mathrm{m}^{-3}$ ) were measured for extracted cores of 204 trees. The interpolated wood density values were applied on the remaining trees based on a calibration equation with pin penetration depth measured using a Pilodyn 6J wood tester (PROCEQ SA, Zürich, Switzerland). Additionally, forest understory trees with a diameter of $2-9.9 \mathrm{~cm}$ were inventoried as described above, within two subplots in each replicate plot. To convert the recorded tree structural data into above-ground plant biomass ( $\mathrm{AGB} ; \mathrm{Mg} \mathrm{ha}^{-1}$ ), the allometric equations developed by Chave et al. (2005) for all forest trees, Wauters et al. (2008) for rubber trees, Asari et al. (2013) for oil palms and Schnitzer et al. (2006) for lianas were applied.

To calculate coarse root and root stock biomass (below-ground plant biomass; $\mathrm{Mg}$ $\mathrm{ha}^{-1}$ ), we used the allometric equations developed by Niiyama et al. (2010) for forest trees, Wauters et al. (2008) for rubber trees and Syahrinudin (2005) for oil palm trees. We added our measurements of small-diameter $(<2 \mathrm{~mm})$ root biomass to the calculated total biomass. Fine root biomass was measured using 10 vertical soil cores $(3.5 \mathrm{~cm}$ in diameter) down to $0.5 \mathrm{~m}$ soil depth, including the organic layer, in each replicate plot. All fine root segments longer than $1 \mathrm{~cm}$ were extracted by washing over a sieve of $200 \mu \mathrm{m}$ 
mesh size (Retsch, Haan, Germany) and separated under a stereomicroscope into live (biomass) and dead fractions (necromass).

Woody coarse debris was analysed within all forest and jungle rubber plots (all woody coarse debris is cleared regularly from monoculture rubber and oil-palm plantations so was not measured at these plots), where snags (DBH $>10 \mathrm{~cm})$ and $\operatorname{logs}$ (mid-point diameter $>10 \mathrm{~cm}$, length $>1 \mathrm{~m}$ ) were also recorded. Three decay stages based on Grove (2001) were used to characterise the woody debris. Biomass of the debris was calculated using the equations by Kauffmann and Donato (2012) and by applying the allometric equation by Chave et al. (2005) for calculation of AGB of undegraded trees. Above- and belowground biomass sampling was conducted using the collection permit 2704/IPH.I/KS.02/X1/2012 recommended by the Indonesian Institute of Sciences (LIPI) and issued by the Ministry of Forestry (PHKA).

\subsubsection{Microbial diversity and biomass}

Sampling of soils for microbial diversity (top 0.5 to $0.7 \mathrm{~m}$ ) was carried out in three of the subplots in each replicate plot. All samples were stored at $-80^{\circ} \mathrm{C}$ until further use. DNA was isolated by employing the PowerSoil DNA isolation kit as recommended by the manufacturer (Dianova, Hamburg, Germany). Subsequently, 16S rRNA gene amplicons of Bacteria and Archaea were generated from the isolated DNA as described by Schneider et al. (2013). The resulting 16S rRNA gene datasets were processed and analysed by employing QIIME 1.8 (Caporaso et al. 2010). Initially, sequences shorter than $300 \mathrm{bp}$, containing unresolved nucleotides, exhibiting an average quality score lower than 25, harbouring mismatches longer than $3 \mathrm{bp}$ in the forward primer, or possessing homopolymers longer than 8 bp and primer sequences were removed. Sequencing noise and potential chimeric sequences were resolved by using Acacia (Bragg et al. 2012) and UCHIME (Edgar et al. 2011) with RDP (Larsen et al. 1993) as reference datasets (trainset10_082014_rmdup.fasta). Operational taxonomic unit (OTU) determination was performed at a genetic divergence of 3\% by using pick_open_reference_otus.py of the QIIME 1.8 package using the Silva NR SSU 119 database version as a reference (Caporaso et al. 2010; Quast et al. 2012). Taxonomic classification was performed with parallel_assign_taxonomy_blast.py against the same database. Unclassified OTUs and OTUs occurring as singletons (sequences observed fewer than 2 times), chloroplasts, and extrinsic domains were removed. OTU tables were subsampled and comparisons were performed at the same surveying effort (Bacteria 6.800 and Archaea 2.000 sequences per 
subplot). Diversity estimates were generated employing alpha_rarefaction.py. The 16S rRNA gene sequences were deposited in the National Center for Biotechnology Information (NCBI) Sequence Read Archive (SRA) under study accession number SRP056374. Microbial diversity sampling was conducted using the collection permit 2701/IPH.I/KS.02/XI/2012 recommended by the Indonesian Institute of Sciences (LIPI) and issued by the Ministry of Forestry (PHKA).

Microbial biomass $\mathrm{C}\left(\mathrm{mg} \mathrm{C} \mathrm{kg}^{-1}\right)$ was measured from soil cores sampled in two of the randomly assigned subplots. The $\mathrm{CHCl}_{3}$ fumigation-extraction method was followed (Brookes et al. 1985; Davidson et al. 1989). A subsample of soil from a core was extracted immediately with $0.5 \mathrm{~mol} \mathrm{~L}^{-1} \mathrm{~K}_{2} \mathrm{SO}_{4}$ (unfumigated) and another subsample was fumigated with $\mathrm{CHCl}_{3}$ for six days and then extracted (fumigated). Organic $\mathrm{C}$ in the extracts was analysed by UV-enhanced persulfate oxidation using a Total Organic Carbon Analyser (TOC-Vwp, Shimadzu Europa GmbH, Duisburg, Germany) with an infrared detector. Microbial biomass $\mathrm{C}$ was calculated as the difference in extractable organic $\mathrm{C}$ between the fumigated and unfumigated soils divided by $\mathrm{k}_{\mathrm{C}}=0.45$ for a six-day fumigation period (Brookes et al. 1985). Microbial biomass sampling was conducted under the same collection permits as those for the soil sampling above.

\subsubsection{Litter macroinvertebrate diversity and biomass}

In each of the replicate plots, three of the randomly assigned subplots were sampled by sieving $1 \mathrm{~m}^{2}$ leaf litter through a coarse $2 \mathrm{~cm}$ mesh-width sieve. 7472 macroinvertebrates were hand-collected from the sieving samples and stored in $65 \%$ ethanol. Specimens were identified to morphospecies and, based on morphology and literature, assigned to one of four feeding guilds: omnivores, detritivores, predators and herbivores. To assess sampling completeness, observed species richness was compared to both extrapolated and rarefied species richness, which revealed that the mean estimated sampling coverage was $56 \%$ and observed species richness was highly correlated with extrapolated species richness (Supplementary Figure 3.2). As such, observed species richness provided representative sampling of real species richness in these tropical agricultural systems. For each of the 7472 animals collected, individual body lengths were measured and then converted to fresh body mass using allometric length-mass regressions from the literature (Supplementary Table 3.1a and b). Community biomass (fresh mass in $\mathrm{mg} \mathrm{m}^{-2}$ ) was then calculated for each of the 32 sampled communities by summing together all individual body masses calculated from length-mass regressions as 
derived from the individually measured body lengths. All organisms were collected based on Permit No. 51/KKH-5/TRP/2014 issued by the Indonesian Institute of Sciences (LIPI) and the Ministry of Forestry (PHKA).

\subsubsection{Arboreal ant diversity and biomass}

Plastic observation plates with two baits of $2 \mathrm{~cm}^{3}$ of tuna in oil and two sponges saturated with $70 \%$ sucrose solution attached were used to sample arboreal ant species (Wielgoss et al. 2010). One plate was tied at breast height on two trees within all five of the subplots in each replicate plot. If there were not two trees in a subplot (often the case in oil palm plantations), the closest trees to the subplot were chosen. Sixty minutes after placing the plates on the trees, the abundance of each ant species present on the plate was recorded and a number of specimens were collected. Ant species abundance at a given plot was defined as the mean of all recorded abundances in a plot. Surveys were conducted in each replicate plot four times between November 2012 and February 2014, between 9:00 am and 11:00 am. No sampling was conducted during or immediately after rain due to a reduction in ant activity in wet conditions. All collected ants (Hymenoptera: Formicidae) were identified to genus using published keys (Fayle, Yusah \& Hashimoto 2014). We identified specimens to species level where possible and assigned the remainder to morphospecies. Body length was measured on up to five individuals from each species at each plot and, using a length-mass regression specific to ants (Gowing \& Recher 1984), an average body mass was calculated for each species. Ant community biomass (mg fresh mass) for each site was calculated by summing the total biomass of each species (abundance $\times$ average species body mass) present. Arboreal ant sampling was conducted using the collection permit 11055/IT3/PL/2012 recommended by the Indonesian Institute of Sciences (LIPI) and issued by the Ministry of Forestry (PHKA).

\subsubsection{Bird diversity and biomass}

Birds were sampled using point counts located in the centre of the replicate plots. All birds detected visually and aurally within the plot were recorded for 20 minutes between 6:00 am and 10:00 am in June to July 2013. The timing of bird data collection alternated between early and late morning and all replicate plots were visited three times. Individuals flying above the canopy were excluded and unfamiliar bird calls were recorded using a directional microphone (Sennheiser ME66). The recordings were compared with an available online bird call database (www.xeno-canto.org) for 
confirmation. Bird species identification in the field follows Mackinnon \& Phillips ${ }^{34}$, but thereafter, species names were translated to Birdlife International nomenclature. Body mass and feeding guilds for each bird species were retrieved from Wilman et al. (2014) and assigned to each individual recorded. Bird species were divided into 3 main feeding guilds: herbivores (granivores, nectivores, and frugivores), predators (insectivores), and omnivores (insectivores and/or granivores, nectivores, and frugivores). Total biomass for each replicate plot was calculated by summing together species-specific body sizes for all individuals sampled.

\subsubsection{Statistical analyses}

To reduce the number of parameters in our models and avoid issues of multicollinearity whilst maintaining as much variability in our predictors as possible, composite variables were calculated using principal components analysis (PCA; 'princomp' function in R (R Core Team 2015) from soil, microclimatic, and plant community parameters. Soil components were created using a combination of soil biochemical properties and soil physical properties (Swaine 1996). Following Swaine (1996), soil physical properties were first composited to create a soil physical component. Including soil texture (\% sand, \% silt, \% clay), soil bulk density, and soil moisture within the soil physical component, the first principal component (PC1) accounted for $53.5 \%$ of the total variation (Supplementary Figure 2.3a). The physical component PCA scores from the PC1 were plotted against the soil physical variables with the highest loadings (> 0.400) in order to ascertain trends within the component (Supplementary Figure 2.3b). \% Sand had negative loading, and \% clay and soil moisture had positive loadings (Supplementary Figure 2.3a), illustrating that the lower PC1 scores represented coarser textured drier soil conditions and the higher PC1 scores represented finer textured wetter soil conditions (Supplementary Figure 2.3b).

The soil physical component was then combined with soil biochemical variables (e.g. soil $\mathrm{pH}, \mathrm{SOC}$, total $\mathrm{N}, \mathrm{C}: \mathrm{N}$ ratio, extractable $\mathrm{P}, \mathrm{ECEC}$, base saturation and $\delta^{15} \mathrm{~N}$ ) (Swaine 1996) in a new PCA. Two composite variables were created representing the soil conditions from the first (PC1) and second (PC2) principal components. PC1 and PC2, combined, accounted for $57.2 \%$ of the total variation (Supplementary Figure 2.3a). Soil PC1 was termed Nutrient Availability and reflects the level of acid and base cations in the soil. The higher PC1 scores represented soil conditions with higher nutrient availability and the lower PC1 scores represented soil conditions with lower soil nutrient availability 
(Supplementary Figure 2.3b). Soil PC2 was termed Soil Organic Matter, as the higher PC2 scores represented soil conditions with higher SOC, lower soil $\mathrm{pH}$, and less decomposed organic matter (as evident by the lower $\delta^{15} \mathrm{~N}$ values) and the lower PC2 scores represented soil conditions with less SOC, higher soil $\mathrm{pH}$, and more decomposed organic matter. PC2 is also dependent on the agricultural management practices that took place in the land-use systems in this study. Soil amendments such as liming and ash from burning during conversion increased soil pH (Allen et al. 2015). Additionally, weeding, herbicide use and harvest export reduced the organic matter input (Kotowska et al. 2015), which in turn resulted in highly decomposed organic matter (i.e., higher $\delta^{15} \mathrm{~N}$ signatures).

Using ranges calculated from the $5^{\text {th }}$ and $95^{\text {th }}$ percentiles for air temperature, humidity and soil temperature, a composited microclimate variable representing Microclimatic Variability was created with a PCA. The PC1 axis accounted for $70.1 \%$ of the total variation (Supplementary Figure 2.3a). Relationships between the microclimatic variables and the PC1 scores illustrated that the PC1 scores were strongly positively correlated with climatic variability (Supplementary Figure 2.3b).

Because plant diversity as well as above- and below-ground plant biomass were highly correlated (90.36\%; Supplementary Figure $2.3 \mathrm{a})$, these variables could not be reliably modelled together as predictors in our path model. Therefore, a composite variable was constructed as described above, combining plant diversity, above- and below-ground biomass in a PCA. The Plant Diversity and Biomass component (PC1) accounted for $94.9 \%$ of the total variation and each individual variable was loaded relatively equally (Supplementary Figure 2.3a and b). The Plant Diversity and Biomass composite variable was clearly defined as the higher the PC1 score, the greater the plant diversity, above- and below-ground biomass (Supplementary Figure 2.3b).

Due to the hierarchical layout of the sampling design, whereby replicate plots were split between two different landscapes, as well as a need for the flexibility to test for interactions between land use and abiotic variables, multilevel generalised path analysis was employed (Shipley 2009). To construct such a model, first the basis set $\boldsymbol{B}_{U}$ of independence claims that are implied by a directed acyclic causal diagram (i.e. a unidirectional box-and-arrow diagram) was identified. To do so, all possible relationships between predictor and response variables were identified. Specifically, direct and interactive effects between land use and all abiotic variables on biotic response variables for ecologically meaningful relationships were allowed for (see Supplementary Figure 2.2). Additionally, only likely bottom-up trophic effects among biotic variables were 
allowed for. Although top-down effects are also likely to play a role in structuring multitrophic communities, here we specifically test for bottom-up effects because we expected the impacts of land-use intensification to be most severe on plant and microbial communities (Chapin III et al. 2000). $\boldsymbol{B}_{U}$ expresses the full set of independence claims (i.e. pairs of variables in the acyclic model with no arrow between them) and implies dependence claims (pairs of variables in the model with a causal arrow joining them). These claims describe the $p_{i}$ probability that variable pairs $\left(X_{i}, X_{j}\right)$ are independent conditional on the variable set, $\boldsymbol{Z}$, which is a direct cause of either $X_{i}$ or $X_{j}$. The probabilities $p_{i}$ for each of the $k$ independence claims in $\boldsymbol{B}_{U}$ were identified using linear mixed effects models. The combined $p_{i}$ of the full model was calculated as

$$
C=-2 \sum_{i=1}^{k} \ln \left(p_{i}\right)
$$

and the $C$ value was then compared to a chi-square $\left(\chi^{2}\right)$ distribution with $2 k$ degrees of freedom (Shipley 2009). The resulting probability, $P$, indicates whether the data depart significantly from what would be expected under such a causal model(Shipley 2009). A model is rejected if the resulting $P$-value is smaller than the specified $\alpha$-level (in this case $\alpha=0.05)$. Therefore, if $P>0.05$ the causal model is not rejected and provides a good fit to the data.

Linear mixed effects models with random intercepts were fitted using the 'nlme' package in R 3.0.1 (R Core Team 2015), with 'landscape’ (i.e., Bukit Duabelas and Harapan locations) specified as a random effect. All variables were mean-centred by subtracting the mean of a given variable from each value of that variable. Assumptions of normality were tested by inspecting the variance structure of each model. As all predictors in the model were mean-centred, unstandardised path coefficients could be interpreted as the degree of change in the response variable for a given unit change in the predictor. However, interaction terms are interpreted differently whereby the coefficient indicates the amount of change in the slope of the regression of the response variable against a predictor (in this case, land-use) following a unit change in the other interacting predictor variable.

Model fit was assessed using the procedure for calculating $R^{2}$ values for generalised linear mixed effects models as outlined by Nakagawa and Schielzeth (2013). This directly indicates the proportion of total variation of endogenous variables explained 
by all significant predictors and their conditioning variables. Standardised coefficients, or $z$-scores, were calculated to assess relative effect sizes of each predictor on endogenous variables by dividing the mean-centred variables by twice their standard deviation (Gelman 2008) so that effects were expressed in units of standard deviation. As such, standardised effects can be directly compared and thus indicate the relative importance of each path.

\subsection{RESULTS AND DISCUSSION}

Conversion of rainforest to rubber and oil palm agriculture imposed predominantly direct impacts $(90 \%)$ on plants, microbes and invertebrate primary and secondary consumers (i.e. detritivores, herbivores, and omnivores) (Figure 2.2; Supplementary Table 2.2). Direct land-use effects on lower trophic levels were stronger than those at higher trophic levels, with relatively weaker direct effects on belowground omnivores and arboreal ants, and almost no direct effects on predator groups (Figure 2.2). The majority of these direct effects $(67 \%)$ were negative and considerably higher in effect size than cascading trophic effects (Figure 2.3a), driving a clear overall decrease in diversity and biomass with conversion of forest to monoculture plantations (Figure 2.2, Supplementary Figure 2.4). These direct effects of land-use intensification most likely result from the intensive application of pesticides and fertilisers, disturbance of resource pools through the clearing of leaf litter, and habitat fragmentation (Fitzherbert et al. 2008). In other studies, heavy fertilisation has been clearly shown to reduce plant diversity and biomass through induced competitive exclusion (Hautier et al. 2009), whereas fertilisation could possibly drive increases in microbial diversity (Tripathi et al. 2012). Interestingly, the number of direct land-use effects steeply declined with increasing trophic level (Figure 2.3b), indicating a shift away from the direct land-use effects hypothesis higher in the food chain. 


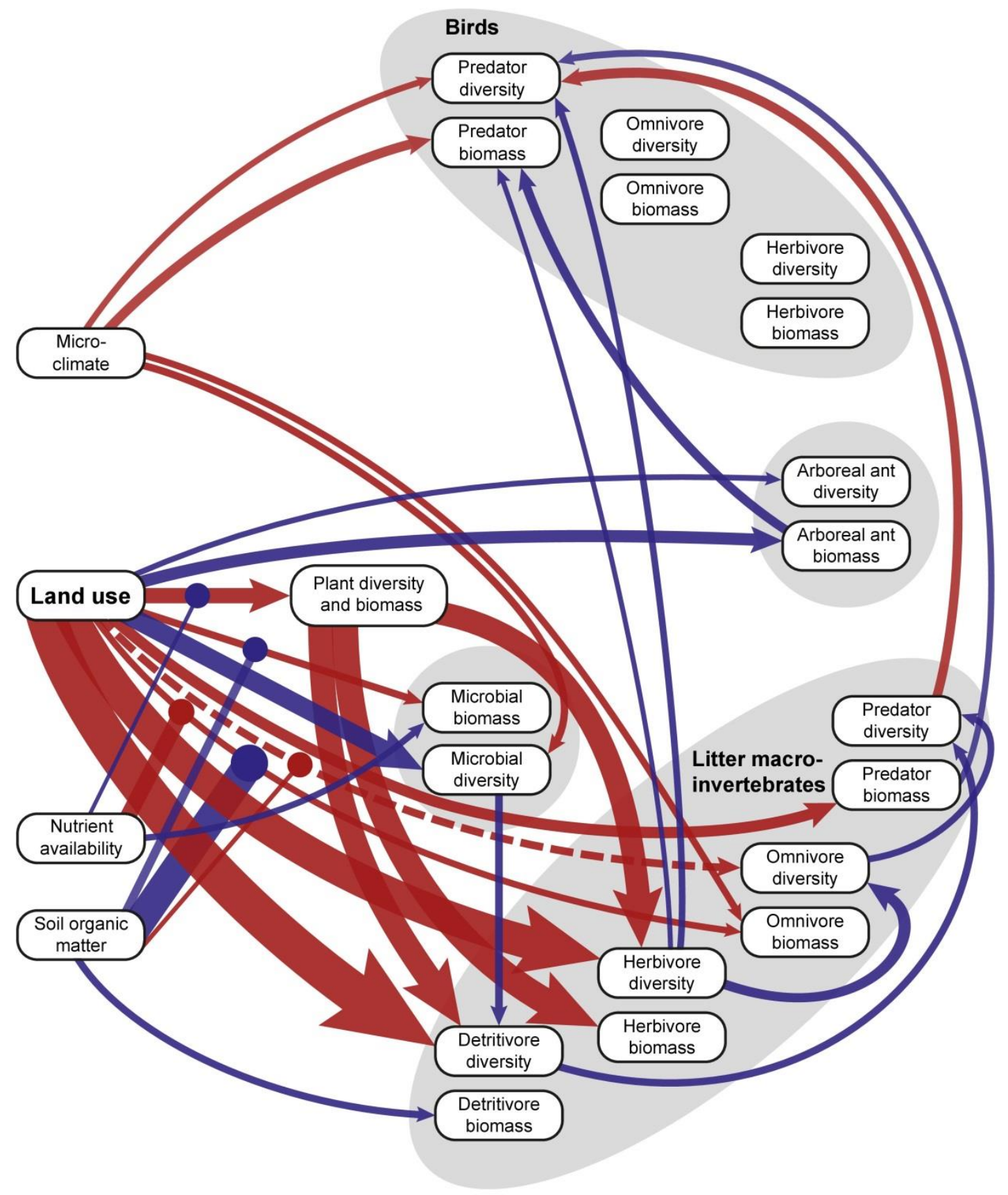

Figure 2.2: Structural equation model testing the hypotheses of direct versus cascading land-use effects in a tropical multitrophic system $\left(\chi^{2}=266.4, \mathrm{df}=276, \mathrm{P}=0.65\right)$. Red and blue arrows denote all significant negative and positive effects, respectively. Arrows ending in circular nodes that intersect the arrows originating from land-use indicate a modification of these effects (i.e., a significant interaction). Arrow widths are proportional to their absolute standardised effect sizes (z-scores) (Supplementary Table 2.2).

Many of the direct land-use effects were moderated by abiotic variables. For example, the negative impact of land-use change on plants was strengthened by soil nutrient availability (Figure 2.2; Supplementary Table 2.2). This interactive effect is derived from management practices, such as fertilisation (Allen et al. 2015), that generate 
high soil nutrient availability and drive even steeper reductions in plant diversity and biomass (Hautier et al. 2009). The frequency of interactive effects of background environmental variables with land-use intensification points to the importance of the landscape context in moderating how severe land-use impacts will be. Despite this, the strongest direct land-use effects acted independently of environmental variables (e.g., on invertebrate detritivores and herbivores), indicating that the overall stronger impacts of land-use intensification (Figure 2.3a) will often override potential interacting environmental variables. Nevertheless, the novel combination of land-use intensification and abiotic variables to explore bottom-up control in taxonomically broad multitrophic systems aided us in explaining a high proportion of variation within our models (ranging from $30 \%$ to $96 \%$, Supplementary Table 2.2). This illustrates the importance of accounting for abiotic effects and how they moderate anthropogenic impacts on ecosystems as they likely propagate upward to higher trophic levels, contributing to the structuring of entire ecosystems.
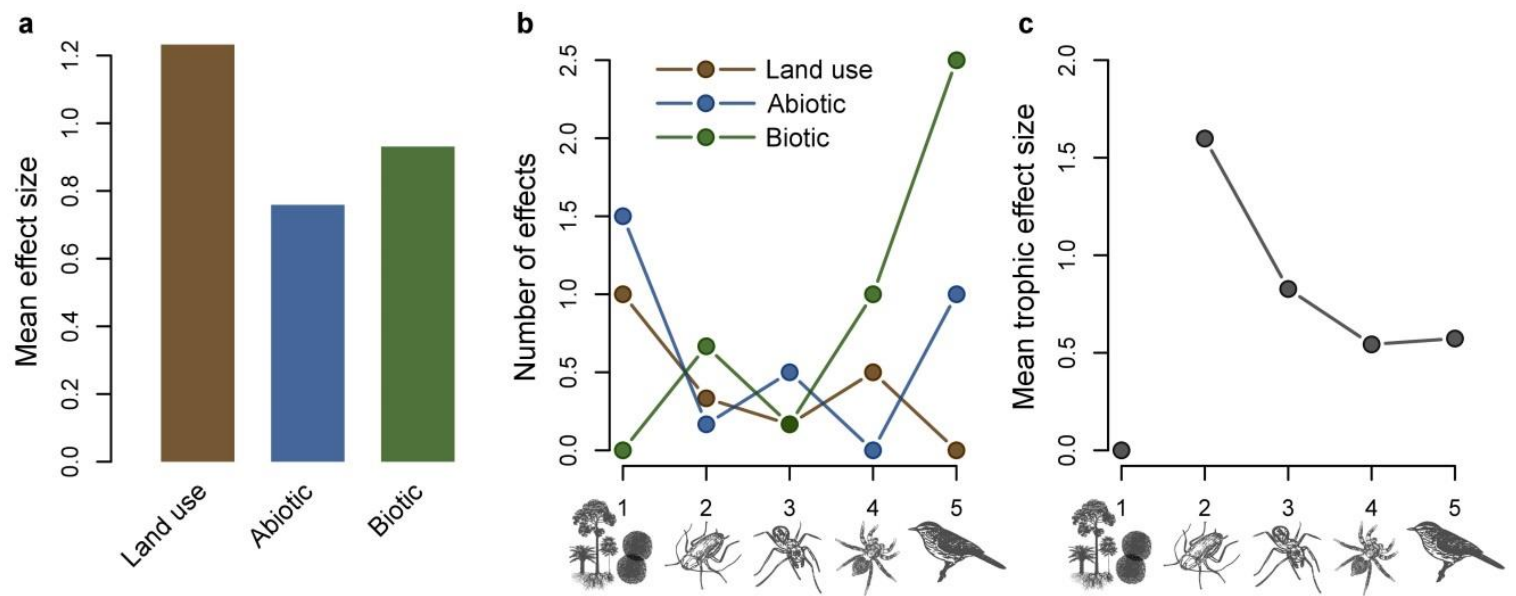

Trophic level

Figure 2.3: Summary of effects in the structural equation model. 'Mean effect size' is the withingroup means of the absolute standardised coefficients. 'Number of effects' shows the total number of land-use, abiotic, and biotic effects at each trophic level, weighted by the number of response variables within each trophic level. 'Mean biotic effect size' gives the mean effect size of all bottom-up trophic effects at each trophic level.

All predatory groups were indirectly affected by land-use intensification (Figure 2.2), with $90 \%$ of effects on these groups propagating via bottom-up trophic cascades. These cascading effects can be explained by strong direct effects of land-use intensification on biomass and diversity of lower trophic levels altering resource availability for higher trophic levels. In stark contrast to direct land-use effects, biotic 
effects (i.e., trophic effects) increased dramatically in number from the lowest to highest trophic levels (Figure 2.3b), clearly indicating a shift in importance from direct to cascading land-use effects with increasing trophic level across a large range of taxa. Interestingly, we found that the mean effect size of bottom-up trophic effects in our model decreased from detritivores to invertebrate omnivores, and to predatory invertebrates and birds (Figure 2.3c). This could be due to greater disturbances of food resources at lower trophic levels (plants and soil microbes) compared with intermediate trophic levels (invertebrate detritivores, herbivores, and omnivores) in higher-intensity agricultural systems. However, this intriguing finding merits further experimental exploration as it could indicate when trophic cascades are likely to be more intense, and how to avoid potentially catastrophic trophic collapses (MacDougall et al. 2013).

Surprisingly, the effects of plant biomass and diversity on vegetation-associated trophic guilds (invertebrate herbivores and detritivores) were negative after controlling for all other variables in our model (Figure 2.2; Supplementary Table 2.2). When the effects of plants on these trophic guilds were analysed without land use as a covariable, however, we found an overall positive effect of plant communities on the diversity of litter invertebrate detritivores (but no significant effects on invertebrate herbivore biomass and diversity) (Supplementary Figure 2.5). Interestingly, plant diversity and biomass had a consistently positive effect on these taxa within the intensive oil palm plantations, but generally negative or null effects in the less intensively managed systems. This finding suggests that at low levels of plant diversity and biomass typically used in experimental studies (Haddad et al. 2009; Scherber et al. 2010; Borer et al. 2012), the relationship between plants and their consumers is most likely to be positive, but this could change markedly at much higher natural levels of diversity, such as in tropical rainforests. These findings could open interesting possibilities for future research into the role of plant diversity on higher trophic levels in biodiversity experiments, calling for the challenging task of incorporating real-world levels of species diversity.

We demonstrate that tropical land-use intensification drives large-scale ecological shifts across trophic levels via direct management impacts and indirect cascading effects. In essence, we find that as one moves upward in the food chain, land-use impacts shift from strong and direct effects to weaker and indirect effects that cascade upward via trophic interactions. At the bottom of the food chain, however, these direct impacts of land-use change are often moderated by environmental variation, collectively emphasising that ecosystems are highly complex and that human activities will yield 
equally complex consequences as ecosystems are altered. Amidst this complexity, the employment of structural equation modelling provides a powerful framework for summarising important effects of land-use intensification in complex systems. Our results elucidate the importance of focusing on conservation strategies that take into account shifting drivers of ecological change across the trophic spectrum.

\subsection{ACKNOWLEDGEMENTS}

We thank the village leaders, local plot owners, PT Humusindo, PT REKI, PT Perkebunan Nusantara VI, and Bukit Duabelas National Park for granting us access and use of their properties. We also acknowledge and thank our permit granting bodies, the Indonesian Institute of Sciences (LIPI) and the Ministry of Forestry (PHKA). We are also especially grateful to all our Indonesian field and laboratory assistants, as well as the rangers within the protected forest areas for their assistance and guidance during field sampling. We also thank Dodo Gunawan from the Meteorological, Climatological and Geophysical Agency of Indonesia for the climate data. This study was financed by the Deutsche Forschungsgemeinschaft (DFG) in the framework of the collaborative German-Indonesian research project CRC990. 
Chapter 3

\section{Consequences of Tropical Land Use for Multitrophic Biodiversity and Ecosystem Functioning}

Andrew D. Barnes, Malte Jochum, Steffen Mumme, Noor Farikhah Haneda, Achmad

Farajallah, Tri Heru Widarto, and Ulrich Brose

\subsection{ABSTRACT}

Our knowledge about land-use impacts on biodiversity and ecosystem functioning is mostly limited to single trophic levels, leaving us uncertain about whole community biodiversity-ecosystem functioning relationships. We analyse consequences of the globally important land-use transformation from tropical forests to oil-palm plantations. Species diversity, density, and biomass of invertebrate communities suffer at least $45 \%$ decreases from rainforest to oil palm. Combining metabolic and food-web theory, we calculate annual energy fluxes to model impacts of land-use intensification on multitrophic ecosystem functioning. We demonstrate a $51 \%$ reduction in energy fluxes from forest to oil-palm communities. Species loss clearly explains variation in energy fluxes, but this relationship depends on land-use systems and functional feeding guilds, whereby predators are the most heavily affected. Biodiversity decline from forest to oil palm is thus accompanied by even stronger reductions in functionality, threatening to severely limit the functional resilience of communities to cope with future global changes.

\subsection{INTRODUCTION}

The transformation from natural ecosystems to agricultural land use and its continued intensification has led to extensive losses in biodiversity and ecosystem services (Gibbs et al. 2010) resulting in the degradation of human well-being (Díaz et al. 2006). The 
transformation of lowland tropical rainforest to oil-palm (Elaeis guineensis Jacq.) plantations has gained more recent attention as an especially severe threat to tropical biodiversity (Koh \& Wilcove 2007; Gilbert 2012). In the last 25 years the total plantation area of oil palm has tripled, with current global estimates of over 15 million hectares (Gilbert 2012), making this crop one of the world's most rapidly expanding forms of agriculture (Fitzherbert et al. 2008). It is now clear that the expansion of oil-palm agriculture is one of the greatest causes of deforestation (Koh et al. 2011; Wilcove et al. 2013) and this threat appears to be increasing without respite as Indonesia, one of the world's leaders in oil palm, makes plans to double production by 2020 (Koh \& Ghazoul 2010). The rapid expansion of such large-scale land-use transformation raises questions about the impending implications for biodiversity and ecosystem functioning in the tropics.

Despite a broad consensus that biodiversity is positively correlated with ecosystem functioning in controlled experiments (Hooper et al. 2005; Cardinale et al. 2006), there are few real-world examples of such biodiversity-ecosystem functioning relationships (Otto et al. 2008; Foster et al. 2011). In fact, until now there have been no studies that explore the relationship between biodiversity and ecosystem functioning in ecosystems undergoing agricultural land-use transformation to oil palm. Thus, our knowledge of this globally important land-use conversion is strongly limited. Furthermore, over the past decade there have been important advances towards multitrophic approaches in research investigating biodiversity-ecosystem functioning relationships (Duffy 2002; Petchey et al. 2004; Cardinale et al. 2006; Schneider, Scheu \& Brose 2012; Schneider \& Brose 2013). Despite these advances, however, we are still substantially limited by the lack of clear approaches to quantify single measures of ecosystem functioning that can be compared among any combination of trophic levels. This has resulted in our inability to directly look at whole-community relationships between entire species assemblages and the respective functional processes carried out in these communities.

Here, we use the total energy flux between functional feeding guilds as a measure of multitrophic ecosystem functioning, as many studies have suggested process rates, such as energy fluxes, to be important proxies for ecosystem functioning (Duffy 2002; Hooper et al. 2005; Srivastava \& Vellend 2005). Depending on the resource pool that the energy flux comes from, these fluxes can be directly related to ecosystem services such as decomposition (de Ruiter et al. 1994; Handa et al. 2014), plant biomass production 
(Tilman, Reich \& Knops 2006; Enquist et al. 2007), or biocontrol through predation (Cardinale et al. 2003). These energy flux calculations are based on metabolic scaling theory (Brown et al. 2004) and principles of food web energy dynamics (de Ruiter et al. 1994). Using individual metabolic rates that are dependent on body mass, environmental temperature, and phylogenetic grouping (de Ruiter et al. 1994; Ehnes, Rall \& Brose 2011), combined with resource-specific assimilation efficiencies (de Ruiter et al. 1993) and energy loss to predation (de Ruiter et al. 1994), we present this energy flux calculation as a unified measure of multitrophic ecosystem functioning (Figure 3.1). Studies that incorporate diversity across trophic levels to test the relationship between biodiversity and ecosystem functioning have predominantly used only biomass as the measure of ecosystem function (Duffy et al. 2007). However, the metabolic activity and thus the energy processing rates of these biomass pools can vary substantially. Integrating over body mass, phylogeny and temperature with their constraints on metabolic rates, and additionally taking into account assimilation efficiencies and loss to predation, our measure of whole-community energy flux inherently incorporates not only biomass, but also other important ecosystem attributes enabling the quantification of emergent functional properties of ecosystems that would otherwise remain undetected. As such, our measure of energy flux provides a comprehensive and robust measure of multitrophic ecosystem functioning that can be utilised for modelling biodiversity-ecosystem functioning relationships for any assemblage of taxonomic groups, whilst incorporating multiple ecological functions.

In the tropical lowland rainforests of Sumatra, Indonesia, which have been undergoing vast land-use transformation to oil palm (Koh et al. 2011), we quantify the impacts of this transformation ranging from tropical secondary rainforest, jungle rubber, and intensively managed rubber, to oil palm. We utilise data gathered from 32 sites on Sumatra, Indonesia, comprising 2415 populations of 871 species. Firstly, we investigate the biodiversity value of jungle rubber, conventional rubber, and secondary forest compared to oil-palm agriculture by comparing observed species richness, density and biomass of litter-associated macro-invertebrate communities across these systems. Secondly, as a multitrophic measure of the rate of ecosystem processes carried out by these communities, we calculate total solid fresh-mass energy flux in a system by incorporating community metabolism (Ehnes et al. 2014), resource-specific assimilation efficiencies and biomass loss to predation (de Ruiter et al. 1994) into whole-community energy flux equations (Figure 3.1). This provides a quantitative measure of multitrophic 
ecosystem functioning, defined here as the total flux of energy from any resource pool to consumer trophic levels. Additionally, this measure can be attributed to specific functional feeding guilds within communities to look for patterns in ecosystem functioning at different trophic levels. Using the energy-mass flow conversion (Peters 1983), we express energy flux as kilograms per hectare, per year, and explore the relationship between total species diversity and energy flux, distinguishing among four transformation systems to test for land-use dependent biodiversity-ecosystem functioning relationships. Our results demonstrate strong losses in species diversity which in turn predicts reductions in whole-community energy fluxes. However, these reductions are strongest in oil-palm systems, suggesting that land-use conversion from forest to oil palm causes disproportionally strong losses in multi-trophic ecosystem functioning.

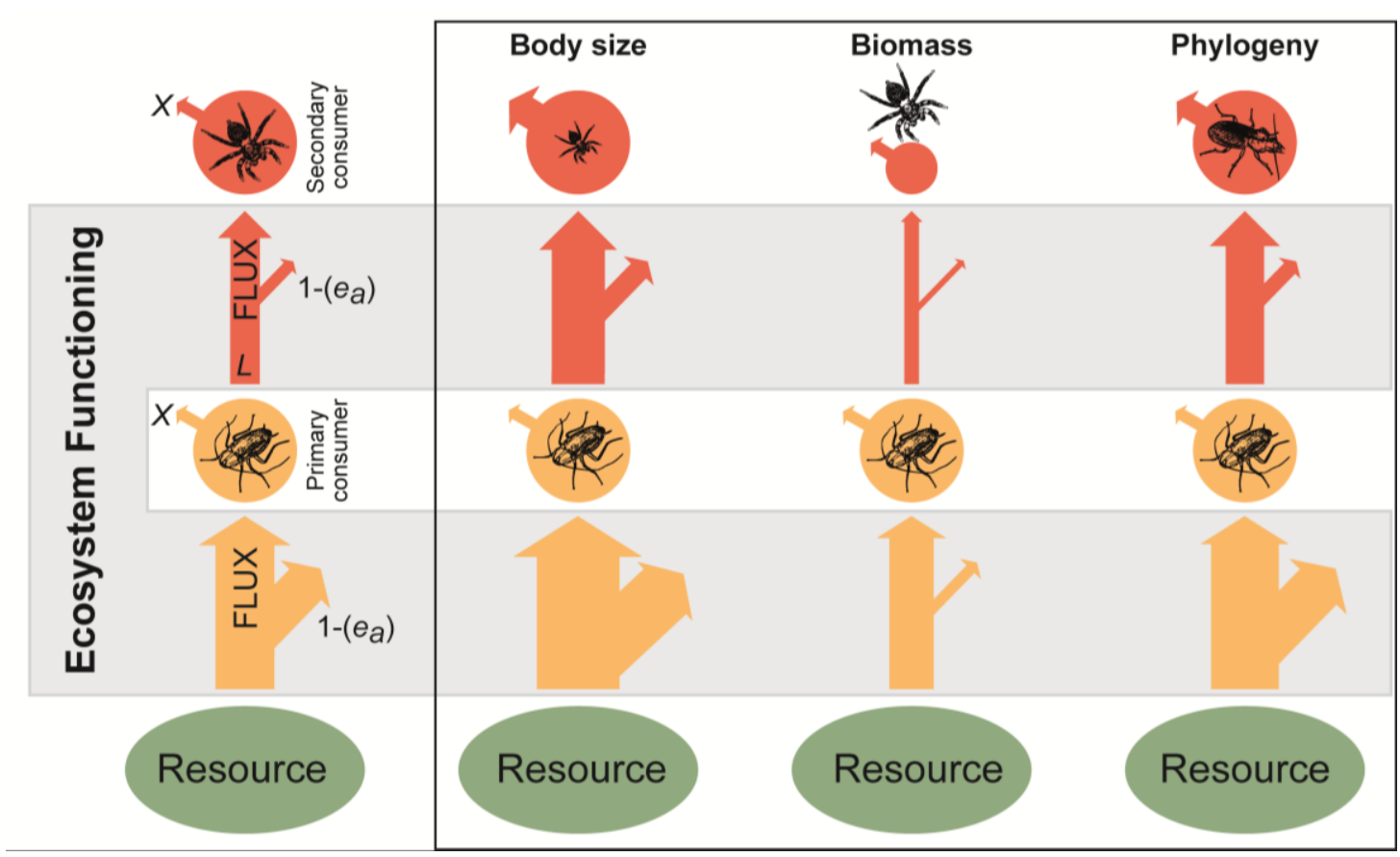

Figure 3.1: Energy fluxes along a conceptual food chain as a measure of multitrophic ecosystem functioning. Energy flux between two nodes is calculated as , where F is the total energy flux into the network node of a feeding guild (vertical red and yellow arrows), $\mathrm{e}_{\mathrm{a}}$ is the diet-specific assimilation efficiency (denoted by diagonal arrows arising from the flux arrows), $\mathrm{X}$ is the perunit-mass metabolic demand of the feeding guild (which is non-linearly dependent on body sizes, temperature, and phylogeny), and $\mathrm{L}$ is the loss to predation from the node (for the yellow node, this is equal to the flux to the red secondary consumer node). Here, we demonstrate three examples where changes in mean body size (size of black animal icons), biomass (diameter of red and yellow circles), or phylogeny (black animal icons) on any trophic level (here, demonstrated by the secondary consumer guild) can result in non-proportionally altered total energy flux (sum of all arrow widths in the food chain). 


\subsection{METHODS}

\subsubsection{Study site and sampling design}

Sampling took place in the Jambi province of Sumatra, Indonesia, a region known as a hotspot for biodiversity, but that has also already undergone extensive deforestation (Sodhi et al. 2004; Wilcove et al. 2013). In the second half of the last century, Sumatra's forests have experienced vast transformation to rubber and oil palm monocultures (Wilcove \& Koh 2010; Laumonier et al. 2010). This large-scale land-use conversion has left Sumatra with a very limited area of natural forest mainly restricted to national parks and even here, where logging has been reduced, it has not come to a complete halt (Gaveau, Wandono \& Setiabudi 2007). This severe and extensive land-use transformation, that has progressed already further than in most other tropical landscapes, makes Sumatra a unique and ideal example system for studying the impacts of land-use conversion on biodiversity and ecosystem functioning.

We sampled secondary rainforest, jungle rubber, rubber and oil-palm systems, replicated eight times across two landscapes $(n=32)$ (Supplementary Figure 3.1). Sites were selected by first looking for landscapes in the Jambi province that still contained secondary rainforest. Secondly, we identified all lowland areas with little or no slope and then randomly selected two landscapes with 16 sites each. Among all of the 32 sampling sites, we maintained a minimum distance of $120 \mathrm{~m}$ to insure independence of the epigaeic invertebrate communities sampled. The secondary-forest regions lie within two protected areas, Bukit Duabelas National Park and Harapan Rainforest, and represent the least impacted land-use system. Jungle rubber-forest stands with a high percentage of rubber trees that are still regularly harvested—represents a low-impact agroforestry system (Gouyon et al. 1993). Rubber and oil-palm plantations serve as locally common (Laumonier et al. 2010) high-impact monocultures. The 32 sites were carefully selected so that they were all of a similar age and from equal elevations close to sea-level. All agricultural systems (jungle rubber, rubber, oil palm) were treated and harvested by their owners with intensities typical for the respective transformation system.

\subsubsection{Animal sampling and calculation of response variables}

Animal sampling took place between early October and early November 2012. All organisms were collected based on Permit No. 51/KKH-5/TRP/2014 issued by the Indonesian Institute of Sciences (LIPI) and the Ministry of Forestry (PHKA). In all 32 of 
the $50 \times 50 \mathrm{~m}$ sites, we sampled once in each of three $5 \times 5 \mathrm{~m}$ sub-plots by sieving the leaf litter from $1 \mathrm{~m}^{2}$ through a coarse sieve of $2 \mathrm{~cm}$ width mesh. 7472 macro-invertebrates were hand-collected from the sieving samples and stored in $65 \%$ ethanol. Specimens were identified to morphospecies and assigned to one of four feeding guilds: omnivores, detritivores, predators and herbivores, based on morphology and literature.

Because biodiversity studies always suffer from under-sampling and correlation of sample size with species richness, we compared observed species richness to both extrapolated and rarefied species richness, calculated in the 'vegan' package in $\mathrm{R}$ ( $\mathrm{R}$ Core Team 2015), to assess the accuracy of our species sampling effort. To extrapolate sampled species richness, we used the non-parametric 2nd order jacknife estimator (Brose, Martinez \& Williams 2003) to calculate extrapolated species richness from the three $1 \mathrm{~m}^{2}$ sub-samples at each of the 32 sites, revealing an estimated mean sampling coverage of $56 \%$ (SD of $\pm 2.393 \%$ ) making the $2^{\text {nd }}$ order jacknife estimator the most accurate extrapolation method (Brose et al. 2003). Additionally, we calculated samplebased rarefaction, whereby rarefaction curves were calculated for each of the 32 sampled sites and then cut off at the sample size of the smallest sample (40 individuals). Because of the very high attrition of data during the rarefaction procedure (a total of 6192 out of 7472 individuals, or $83 \%$, were removed), the rarefied species richness yielded very little resemblance to observed species richness when comparing across transformation systems, resulting in almost no pattern of rarefied richness among transformation systems (Supplementary Figure 3.2). The jacknife2 extrapolated species richness, however, was extremely closely correlated with observed species richness (Pearson's $\rho=0.993$ ) patterns among transformation systems (Supplementary Figure 3.2), suggesting that our observed species richness did in fact accurately capture realistic patterns in total species diversity across the land-use transformation systems.

For each of the 7472 animals collected, we measured individual body length to an accuracy of $0.1 \mathrm{~mm}$ using stage micrometres. We then converted all measured individual body lengths to fresh body mass using length-mass regressions and, where necessary, dry mass-fresh mass relationships from the literature (Supplementary Table 3.1), yielding an estimated fresh mass in mg for every collected individual. Where family-specific relationships were not available or animal body lengths in our collection fell outside of the size ranges of published regressions, we then used regressions from higher-order taxonomic groupings. For heavily damaged individuals that could not be measured for body length, we assigned these individuals a fresh body mass from the median body mass 
of all animals from the same species or order where only one individual of that species was collected. We then calculated community biomass ( $\mathrm{mg}$ fresh mass $\mathrm{m}^{-2}$ ) for each of the 32 communities by summing together all individual body masses calculated from length-mass regressions as derived from the individually measured body lengths.

We calculated individual metabolic rates for all 7472 animals using body masses, temperature, and phylogeny (Ehnes et al. 2011) (Supplementary Table 3.2). Temperature was measured over a period of at least 2.5 months at $30 \mathrm{~cm}$ depth below the soil surface in each site and averaged for each transformation system in each of the two landscapes. From this, community metabolism was calculated by summing together all individual metabolic rates within each of the 32 sites, providing the total metabolic demand for each of the 32 communities. Using diet-specific assimilation efficiencies (de Ruiter et al. 1993), energy loss to predation and community metabolism, we analytically calculated energy fluxes for each of these communities (de Ruiter et al. 1994) using the formula

$$
F=\frac{1}{e_{a}} \cdot(X+L)
$$

where $F$ is the total energy flux into the network node of a feeding guild, $e_{a}$ is the dietspecific assimilation efficiency, $X$ is the metabolic demand of the feeding guild, and $L$ is the loss to predation that the feeding guild is subjected to (Figure 3.1 and Supplementary Methods 3.1). In order to calculate the fluxes between the functional feeding guilds, we constructed a general network of feeding relationships (link structure in Figure 3.3) that represents a null model for an energy network structure where no active preferences are assumed. We assumed that, of our four functional feeding guilds, energy fluxes to predators were split up equally into the three animal guilds below them. Energy fluxes to detritivores and herbivores were assumed to come from only detritus and plant material, respectively. Omnivores were assumed to receive energy in equal 25\% proportions from the other three functional feeding groups (predators, detritivores and herbivores, making $75 \%$ ) and the remaining $25 \%$ from both plant and detritus material combined (Supplementary Methods 3.1).

To assess how these assumptions of feeding preferences might affect the calculations of total energy fluxes, we reconstructed the energy networks so that omnivores were assumed to only consume plant and detritus material (50\% derived from each) but with no energy derived from animal material. We then recalculated total energy 
fluxes and found an overall decrease of up to 54\%, which appeared to be highly consistent among the different land-use transformation systems. This consistency between models was especially evident after calculating the loss of energy flux in the three agriculturally used systems compared with the forest system, demonstrating a maximum of only 3\% disparity between the two models (Supplementary Figure 3.3). This sensitivity analysis indicated that our presented method is highly robust in calculating differences in energy fluxes among different systems. Accordingly, the null model was accepted as the simplest model with the least diet preferences assumed. However, we still suggest that studies adopting this method of energy flux calculation should assign feeding preferences with caution, or employ other techniques such as stable isotope analysis to estimate feeding preferences.

\subsubsection{Statistical analyses}

Using mixed effects models (GLMM's), we tested the effects of 'transformation system' and its interaction with functional feeding guild on community responses, with 'landscape' as a random effect. 'Density', 'biomass', and 'community metabolism' were $\log _{10^{-}}$ transformed to meet assumptions of normality and 'species richness' (overdispersed Poisson-distributed data) was modelled on a negative binomial distribution. We additionally explored biodiversity-ecosystem functioning relationships by first testing for linearity of relationships using untransformed data. Once linearity was established, we then tested for the effects of $\log _{10}$-transformed 'species richness' and its interaction with 'transformation system' on 'energy flux' for overall data and repeated again for data from separate feeding guilds. Additionally, because we suspected that our analyses could be affected by spatial autocorrelation, we calculated Moran's I values for each model's residuals and tested for spatial autocorrelation using the Moran's I standard deviate (Dormann et al. 2007) in the 'spdep' package in R 3.0.1 (R Core Team 2015). Results from these tests provided no support for the spatial autocorrelation of variation in any of the response variables tested (all Moran's I test results yielded $\mathrm{p}>0.4$ ).

For all GLMM's, we applied a backwards stepwise selection procedure to obtain the model of best fit, based on the Akaike Information Criterion (AIC). In this procedure, we constructed full models that contained all possible predictors and their interactions ('transformation system' and 'feeding guild' for general community response models; 'species richness' and 'transformation system' for biodiversity-ecosystem functioning models), and compared these full models and the model of the backward selection 
procedure to a null, intercept-only model. The model that yielded the lowest AIC score, with a minimum $\triangle \mathrm{AIC}$ of 2 units, was selected as the model of best fit. All analyses were conducted with the 'nlme' and 'lme4' packages in R 3.0.1 (R Core Team 2015).

\subsection{RESULTS}

\subsubsection{Transformation to oil-palm agriculture leads to biodiversity loss}

Using generalised linear mixed effects models, we show that transformation of tropical rainforest to oil-palm plantations leads to severe losses in species richness (45\% decline), animal density (48\% decline) and biomass (52\% decline) (Figure 3.2a-c and Supplementary Table 3.3), supporting previous studies suggesting that land-use transformation to oil palm poses one of the greatest threats to global biodiversity (Gilbert 2012). Beyond mere diversity effects, land-use transformation altered animal densities and biomass, threatening to not only drive species extinctions but also to eliminate vital ecological functions. The effects of land-use transformation on species richness and animal densities were additionally dependent on functional feeding guilds, with predators decreasing in species richness and density most rapidly (Figure 3.2a-c and Supplementary Table 3.3) as could be expected for higher trophic level feeding guilds (Purvis et al. 2000). Such alteration of higher trophic levels is likely to have severe indirect functional impacts on other functional guilds within the trophic network (Jochum et al. 2012).

\subsubsection{Community metabolism}

Summing up individual metabolic rates, we demonstrate that transformation of forest to oil palm yields a 51\% decrease in community metabolism, with jungle rubber and rubber only $16 \%$ and $10 \%$ below forest levels of community metabolism, respectively. However, all systems yielded significantly higher community metabolism than oil palm (Figure 3.2d and Supplementary Table 3.3). As such, we show that ecosystem energy processing is critically reduced in oil-palm plantations. Interestingly, biomass responses to land-use transformation among feeding guilds were not clearly comparable to responses in community metabolism (Fig 2c, d). This suggests that systematic changes in species composition, body-mass distributions (Supplementary Figure 3.4) and biomass exhibited a complex interaction in determining the functional consequences of land-use transformation. 

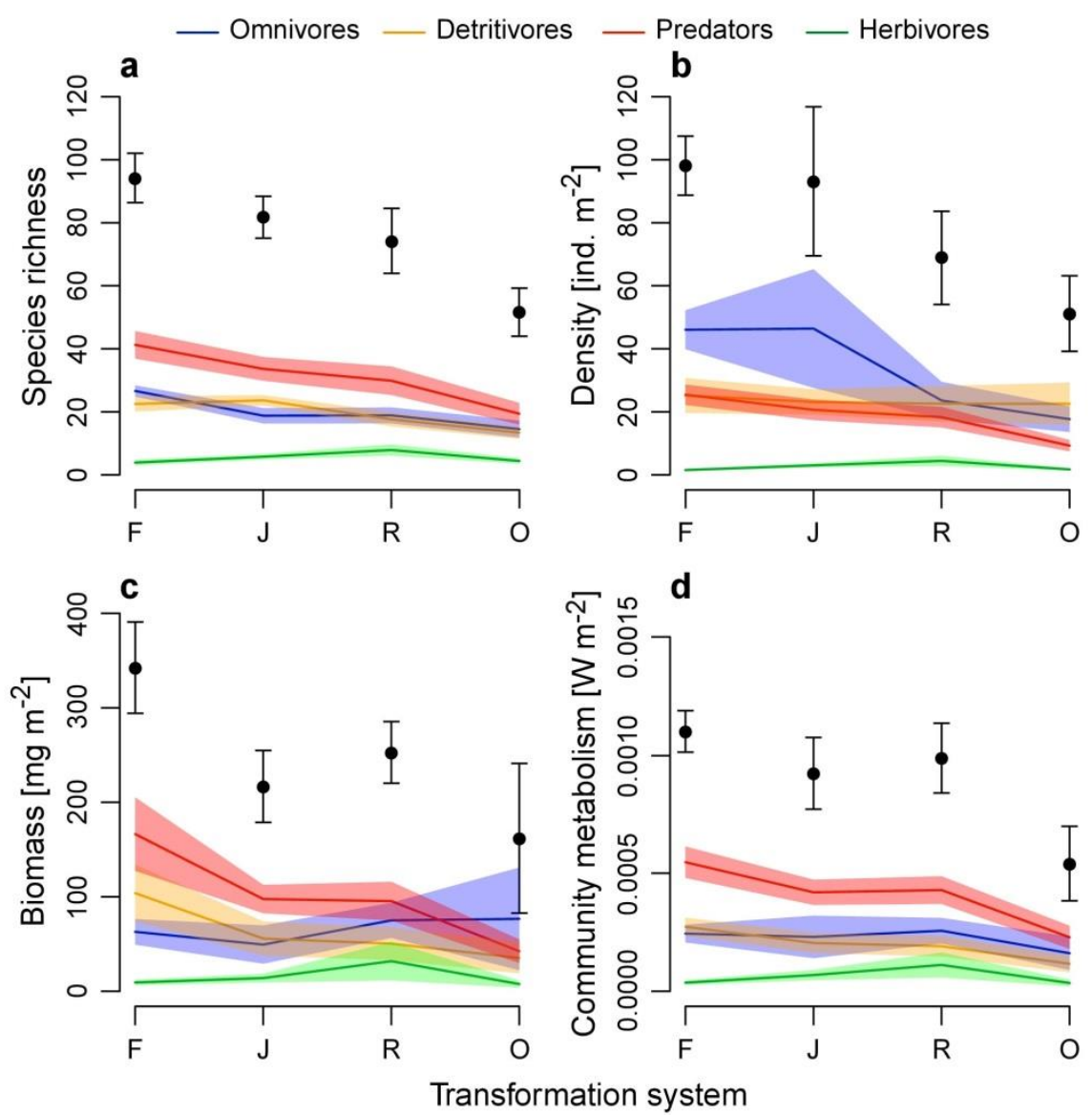

Figure 3.2: Effects of land-use transformation on macro-invertebrate communities.

Mean ( \pm SE, $n=32$ ) species richness (a), density (b), biomass (c), and community metabolism (d) of the total community (black points) and of each functional feeding guild (coloured lines) for the four land-use transformation systems: forest $(\mathrm{F})$, jungle rubber $(J)$, rubber $(R)$ and oil palm $(\mathrm{O})$.

\subsubsection{Whole-community energy fluxes and ecosystem functioning}

Aiming to visualize the complex interplay between community biomass dynamics and energy flux, we constructed energy networks for the four transformation systems (Figure 3.3) based on total energy fluxes as a promising way to quantify multitrophic ecosystem functioning (Figure 3.1). In addition to the general decreases in biomass (node sizes in Figure 3.3) and energy processing rates (arrow widths in Figure 3.3), we also found a systematic shift from predator to omnivore dominance when comparing forest and oilpalm systems. Specifically, we found predator biomass in oil palm yielded only $25 \%$ of their biomass in forest ( 0.424 and $1.664 \mathrm{~kg} \mathrm{ha}^{-1}$, respectively), while the predator-driven energy flux was reduced to $46 \%$ of the energy flux driven by predators in forest (30.697 and $66.816 \mathrm{~kg} \mathrm{ha}^{-1} \mathrm{yr}^{-1}$, respectively). In contrast, omnivore biomass in oil palm was $22 \%$ 
higher than in the forest ( 0.767 compared to $0.629 \mathrm{~kg} \mathrm{ha}^{-1}$ ), while omnivore-driven energy flux in the oil palm was $47 \%$ lower than in forest communities (32.531 compared to $61.900 \mathrm{~kg} \mathrm{ha}^{-1} \mathrm{yr}^{-1}$ ) (Supplementary Table 3.4), suggesting a considerable mismatch of biomass and energy flux, partly dependent on the trophic group in question. In our analyses, this disparity finds its explanation in varying body-mass distributions (Supplementary Figure 3.4) and assimilation efficiencies that strongly modify how biomass translates into total resource assimilation rates (Figure 3.1). These results suggest that biomass, alone, may be an unsuitable proxy for general ecosystem functioning in animal communities.

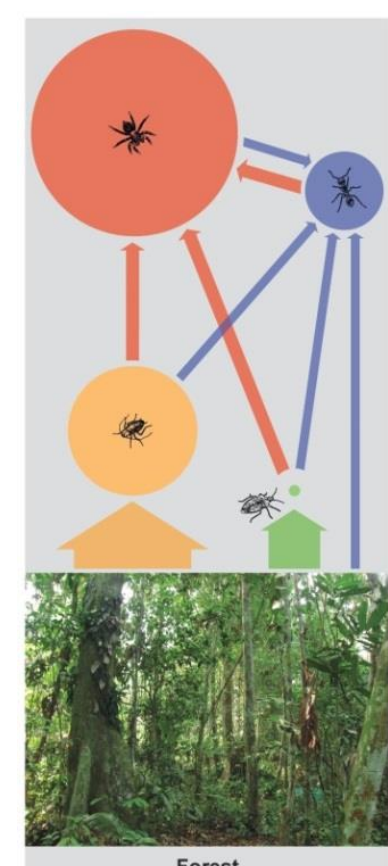

Forest

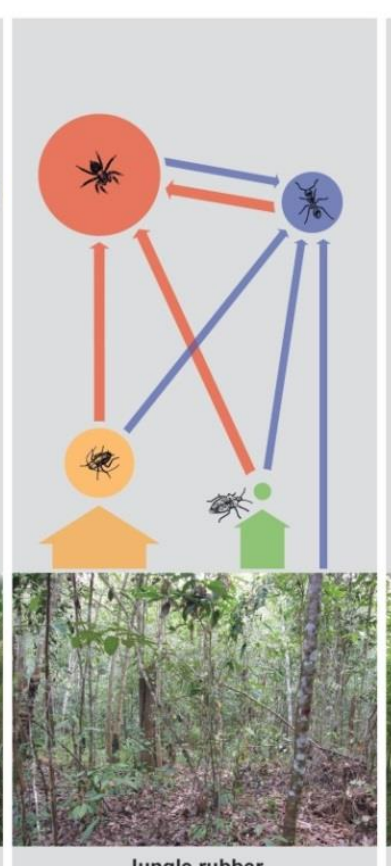

Jungle rubber

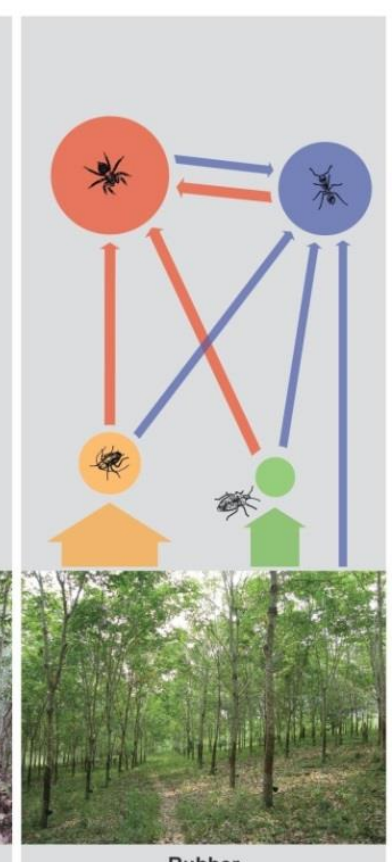

Rubber

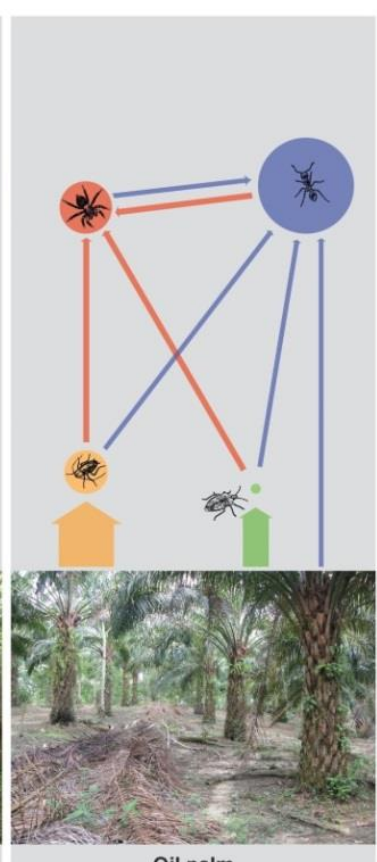

Oil palm

Figure 3.3: Effects of land-use transformation on community energy networks.

Energy networks displaying the relative annual energy flux (coloured arrows weighted by calculated energy flux [kg ha-1 yr-1]) and biomass (coloured nodes weighted by total biomass) among the functional feeding guilds: predators (red), omnivores (blue), detritivores (yellow), and herbivores (green). Each panel represents an energy network for one of the four land-use transformation systems. 

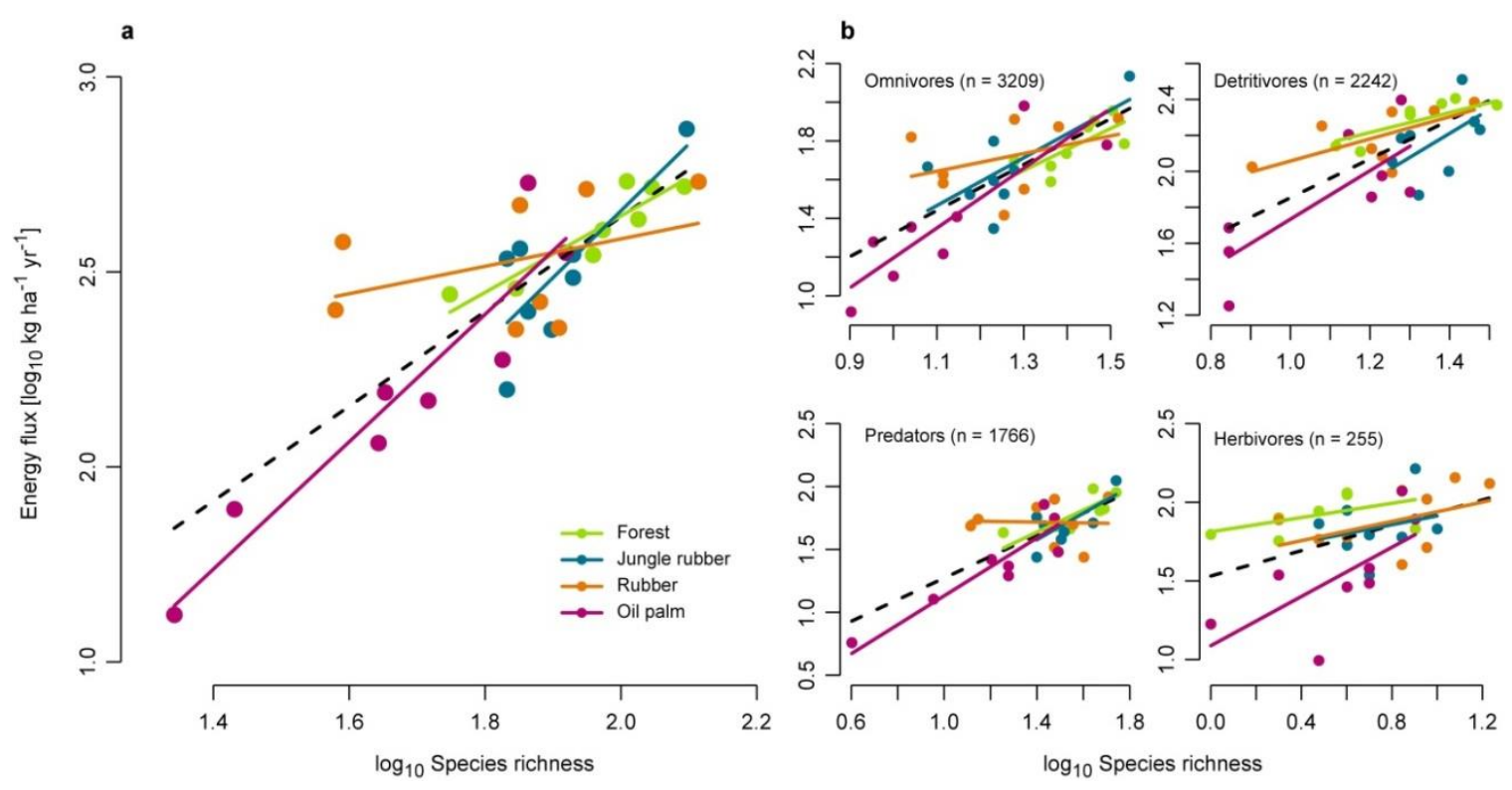

Figure 3.4: Relationship between species richness and community energy fluxes.

Linear mixed effects models for (a) entire communities and (b) separated into functional feeding guilds. Black dashed lines denote overall model fits and coloured lines indicate different land-use transformation systems.

\subsubsection{Multitrophic biodiversity-ecosystem function relationships}

Until now, most studies investigating biodiversity-ecosystem function relationships have focused on single trophic levels (Ives, Cardinale \& Snyder 2004; Balvanera et al. 2006). We present a new approach to easily quantify multitrophic ecosystem functioning, requiring only information on body mass, phylogeny, temperature, and assimilation efficiencies to overcome previous limitations in biodiversity-ecosystem functioning research. Utilising this approach, we also investigated the relationship between species richness and ecosystem functioning, identifying a clear linear positive effect of diversity on total energy flux (Figure 3.4a and Supplementary Table 3.5). The relationship between diversity and energy flux was dependent on land-use transformation system, whereby oil palm and jungle rubber showed the strongest decrease in energy flux per unit loss in species richness (Figure 3.4a and Supplementary Table 3.5). Our results suggest that each loss of species in oil palm and jungle rubber therefore would be followed by proportionately higher losses in energy flux, compared with equal species losses in forest and rubber. We found the same pattern as in the overall trend for the predator group, which showed transformation system-dependent relationships between species richness and energy flux (Figure 3.4b). However, for omnivores, detritivores and herbivores there was a linear effect of diversity on energy flux driven by these groups, but this effect was 
independent of transformation system (Figure 3.4b and Supplementary Table 3.5). This implies that studies focusing on single trophic levels, or even specific species, may fail to detect the alteration of ecosystem processes resulting from land-use transformation. These results call for a wider application of multitrophic approaches that not only measure one ecosystem property, such as total productivity or decomposition, but that also aim to assess whole-community ecosystem processes such as total energy flux.

\subsection{DISCUSSION}

Our study reflects previous findings that the transformation of forest systems to oil palm has severe impacts not only on single animal populations, but also on communities as a whole. In particular, species richness and animal biomass are most significantly affected. Furthermore, jungle rubber and rubber appear to represent intermediate steps in land-use intensification. Their higher levels of biodiversity and ecosystem functioning indicate that they potentially provide higher ecological value than oil palm. As such, these rubber landuse systems could present economically viable, lower intensity land-use alternatives.

By taking a multitrophic ecosystem functioning approach we demonstrate that, at the community level, species loss leads to a direct linear decrease in ecosystem functioning. This means that any species loss will be followed by a proportionate loss in function, and this relationship becomes proportionately stronger in more intensive transformation systems such as oil-palm plantations. Thus, every one of the few species in high-intensity land-use systems is functionally more important than species in lowintensity systems where functional redundancy is likely to be higher (Laliberté et al. 2010). Without explicit consideration of multiple trophic levels, such emergent properties are likely to be overlooked. Our study demonstrates the crucial implications of tropical land-use intensification for biodiversity and ecosystem functioning across multiple trophic levels, suggesting that these globally important impacts will likely resonate beyond previously explored trophic boundaries. 


\subsection{ACKNOWLEDGEMENTS}

We thank Megawati, Rizky Nazarreta, Keisha Disa Putirama, and Rosario Reza Valentino Lasse for assistance in the field and laboratory. Roswitha Ehnes provided additional metabolic rate regression parameters and Christian Guill assisted in solving energy-flux equations. Ana Meijide, Alexander Knohl, Oleg Panferov and team provided climate data. We also thank the village leaders, local site owners, PT REKI, and Bukit Duabelas National Park for granting us access to their properties. This study was financed by the Deutsche Forschungsgemeinschaft (DFG) in the framework of the collaborative German Indonesian research project CRC990. 
Chapter 4

\section{Functional Diversity and Stability of Litter- Invertebrate Communities Following Land-Use Change in Sumatra, Indonesia}

Steffen Mumme, Malte Jochum, Ulrich Brose, Noor Farikhah Haneda, and Andrew D. Barnes

\subsection{ABSTRACT}

Tropical land-use intensification is rapidly increasing in regions that harbour high levels of biodiversity, thus posing a serious threat to the stability and resilience of tropical ecosystems and the important ecosystem services that they provide. We compared functional group richness and functional dispersion in litter-invertebrate communities among four different land-use systems, ranging in intensity from primary degraded lowland forest to oil-palm agriculture in two landscapes on Sumatra, Indonesia. We then investigated the consequences for functional stability and community resilience by calculating functional redundancy and response diversity of sampled communities. From primary degraded forest to intensively managed oil-palm systems, we found a $46 \%$ decrease in species richness and a $48 \%$ reduction in density, but weaker effects on functional group richness and an increase in functional dispersion. Although we detected no significant alteration of response diversity, functional redundancy of litter-invertebrate communities decreased clearly by losing $37 \%$ of functionally redundant species due to land-use change. Our results indicate that land-use change, from tropical rainforest to oilpalm agriculture, can alter both taxonomic and functional diversity of litter-invertebrate communities, resulting in the loss of functional redundancy and thus functional stability of these ecosystems. However, we also show that land-use systems of intermediate management intensity, such as jungle-rubber agroforestry, could serve as reservoirs of functional diversity and stability in monoculture-dominated production landscapes. 


\subsection{INTRODUCTION}

Land-use change imposes strong impacts on biodiversity, which in turn can have important implications for the stability and resilience of natural ecosystems (Foley et al. 2005; Flynn et al. 2009; Laliberté et al. 2010). Tropical forests, which harbour particularly high levels of biodiversity, are increasingly threatened by land-use conversion and intensification to agricultural cash crop plantations (Koh \& Wilcove 2007; Turner et al. 2008; Gibson et al. 2011). In particular, oil palm (Elaeis guineensis) is one of the fastest expanding crops globally (Fitzherbert et al. 2008), with Indonesia and Malaysia together responsible for $90 \%$ of global palm-oil production (Sheil et al. 2009). In addition, monoculture rubber (Hevea brasiliensis) plantations have expanded rapidly in the region, with an increase in area of $32 \%$ over the last decade (Wilcove et al. 2013). This expansion of monoculture crops has been largely associated with the conversion of diverse tropical forests and is therefore directly linked to large-scale losses in biodiversity and ecosystem functioning (Koh \& Wilcove 2007; Wilcove et al. 2013).

Biodiversity loss resulting from monoculture expansion in tropical landscapes has been shown to span many taxonomic groups (Fitzherbert et al. 2008; Senior et al. 2012). These impacts are especially detrimental for forest-specialist species that are particularly sensitive to the loss and fragmentation of tropical rainforest landscapes (Koh \& Wilcove 2008). The high-intensity management of oil-palm dominated systems makes them an ecologically poor substitute for tropical forest ecosystems as they support relatively low species diversity (Fitzherbert et al. 2008). However, there is still very limited knowledge of the consequences of rainforest transformation to oil-palm and other tropical plantation forestry for the stability of ecosystem functioning, and how intermediate levels of landuse intensity could maintain species diversity and functional stability in these altered landscapes.

Over the last 20 years, a considerable body of research has established the importance of functional diversity in discerning the responses of biological communities to global change drivers (Tilman et al. 1997a; Cadotte, Carscadden \& Mirotchnick 2011). Functional diversity is the diversity of functional identities of organisms in a community based on morphological, behavioural, or life-history traits, which are often directly linked to an organism's ecological performance (Petchey \& Gaston 2006; Villéger, Mason \& Mouillot 2008; Gagic et al. 2015). As such, functional diversity can be directly related to ecological attributes such as the functional stability or the resilience of communities, 
depending on the trait complexes measured (Laliberté et al. 2010). Various approaches for assessing functional diversity have been proposed, with a clear development of measures to deal with specific research questions. Functional group richness - the total number of species groups composed of functionally similar species-has been widely used for quantifying the functional heterogeneity of communities (Symstad 2000; Lanta \& Lepš 2007). However, because functional group richness is a rather coarse measure of functional diversity (Petchey \& Gaston 2006), other measures such as the multivariate distance-based metric functional dispersion (Laliberté \& Legendre 2010) have been developed to detect more fine-scale patterns in functional heterogeneity.

A particularly important application of functional diversity is its utilisation for assessing community functional stability and resilience (Laliberté et al. 2010). This approach employs functional grouping to quantify the absolute number of taxonomic species within functional effect groups (i.e., groups of species sharing similar traits important for ecosystem functioning) to assess functional redundancy, and utilises functional dispersion within functional response groups (groups of species sharing similar traits that determine responses to environmental disturbances) to calculate response diversity. Calculated in this way, functional redundancy evaluates the risk of losing ecosystem functions resulting from the loss of all species within a functional group, providing an indication of the functional stability of an ecosystem, rather than the rate of ecosystem functioning per se. On the other hand, response diversity is a measure of the heterogeneity of responses in species assemblages within functional groups, thus giving insight into the resilience of communities regarding future disturbances. These measures clearly provide a powerful estimation of community functional stability and resilience. Although previous studies have investigated patterns in functional diversity for specific taxa within tropical agricultural landscapes (e.g., F. A. Edwards et al., 2014; Gray et al., 2014), to our knowledge there have been no studies quantifying these general measures of stability and resilience in highly diverse tropical animal communities spanning multiple higher-order taxa.

In this study, we assessed the consequences of land-use change for the stability and resilience of diverse tropical litter arthropod communities by evaluating changes in their functional trait composition following conversion of tropical rainforest to plantation agriculture. While Chapter 3 demonstrates how this land-use change alters the diversity and functional efficiency of these communities, this study provides new insight into the future certainty of maintaining these ecological functions. To detect differences in 
functional diversity of species assemblages among land-use systems, we calculated functional group richness and functional dispersion (Laliberté \& Legendre 2010). Using these results we evaluated 1) community stability by calculating functional redundancy and 2) community resilience to future disturbances by calculating response diversity, across four tropical land-use systems of varying intensities. By comparing oil-palm and rubber monoculture systems to primary degraded forest (as defined by Margono et al. (2014)) and a less intensively managed agroforestry system, i.e., jungle rubber (Gouyon et al. 1993), we established the impacts of tropical land-use change on functional diversity, redundancy, and community resilience. Additionally, we discerned how intermediate intensities of agroforestry can serve as potential reservoirs of functional diversity and maintain community functional stability in anthropogenic landscapes.

\subsection{METHODS}

\subsubsection{Study system}

Sampling took place between October and November 2012 in the Jambi Province, Sumatra, Indonesia; an area formerly known to have one of the largest tracts of rainforest in Southeast Asia. Today, the landscape is characterised by a mosaic of agroforestry plantations and primary degraded lowland rainforest. The sampling regions are at 0-400 $\mathrm{m}$ above sea level, with a mean annual temperature of $25^{\circ} \mathrm{C}$ and an annual precipitation of 2000-3000 mm (Murdiyarso et al. 2002). The region has suffered one of the highest deforestation rates worldwide, predominantly due to conversion of land to plantation agriculture (Abood et al. 2014).

\subsubsection{Sampling design}

Macro-invertebrate communities were sampled in four land-use systems: primary degraded forest, jungle rubber, monoculture rubber ( $7-17$ years old), and oil palm ( $9-$ 16 years old), replicated four times in each of the two landscapes, Bukit Duabelas and Harapan ( $\mathrm{n}=32$ ) (Figure 4.1). We sieved $1 \mathrm{~m}^{2}$ of leaf litter, using a coarse $2 \mathrm{~cm}$ mesh width, within each of three randomly placed $5 \times 5$ m subplots located within the $50 \times 50$ $\mathrm{m}$ sampling sites (Figure 4.1). Macro-invertebrates were hand-collected from the litter sieves, stored in $75 \%$ ethanol and transported to the laboratory for identification and trait measurements. Macro-invertebrates were collected under Permit No. 
2695/IPH.1/KS.02/XI/2012 issued by the Indonesian Institute of Sciences and the Ministry of Forestry.

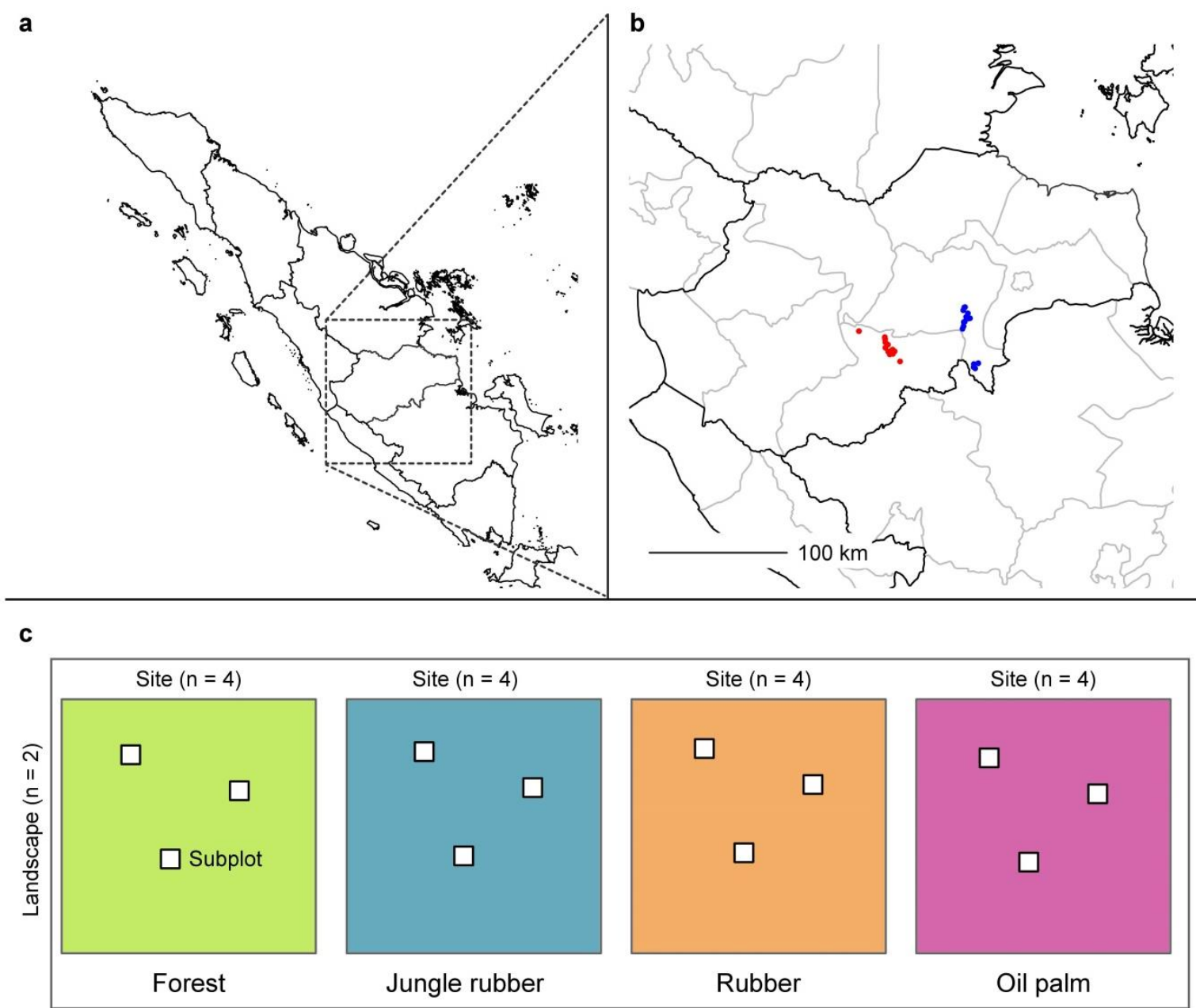

Figure 4.1: Study-site location and sampling design. Sumatra, Indonesia, (a) and the study region in Jambi Province (b) with red and blue circles representing the 16 sites in Bukit Duabelas landscape and the 16 sites in Harapan landscape, respectively. The study design (c) comprises 32 sites with 4 replicates of each of the four land-use systems in each of two landscapes.

\subsubsection{Animal identification and trait measurements}

All animals were identified to morphospecies based on consistent morphological characteristics. We measured four different traits for every individual: Body mass, dispersal capacity, eusociality and feeding type. These traits were defined as 'response traits', 'effect traits', or both (Table 4.1), where response traits determine the animal's response to environmental changes and effect traits determine the animal's effect on environmental processes (Suding et al. 2008). One important goal of this study was to assess functional diversity, redundancy and response diversity across taxonomically broad 
communities without bias toward any particular taxonomic groups. As such, we had to carefully choose traits that would be highly meaningful response and effect traits for all organisms that were sampled. For example, traits such as leg length would have very different functional implications for orthopterans than for diplopods, compared to the highly universal traits we used such as body size.

Table 4.1: Response and effect traits used in the analyses. Dispersal capacity was separated into three categories of no wings, no legs (NN); legs, no wings (LN); legs and wings (LW). Body masses were calculated from individual body lengths using allometric length-mass equations (Supplementary Table 3.1).

\begin{tabular}{|c|c|c|c|c|}
\hline Trait & Source & $\begin{array}{l}\text { Effect } \\
\text { trait }\end{array}$ & $\begin{array}{c}\text { Response } \\
\text { trait }\end{array}$ & Measurement \\
\hline Body mass & Morphology & yes & yes & $\begin{array}{l}\text { Continuous: } \\
\text { body length }[\mathrm{mm}]\end{array}$ \\
\hline Dispersal capacity & $\begin{array}{l}\text { Morphology, } \\
\text { literature }\end{array}$ & no & yes & $\begin{array}{l}\text { Categorical: } \\
\text { NN, LN, LW }\end{array}$ \\
\hline Feeding type & $\begin{array}{l}\text { Morphology, } \\
\text { literature }\end{array}$ & yes & yes & $\begin{array}{l}\text { Categorical: } \\
\text { predator, herbivore, } \\
\text { omnivore, detritivore, } \\
\text { fungivore, parasite }\end{array}$ \\
\hline Eusociality & Literature & yes & yes & $\begin{array}{l}\text { Categorical: } \\
\text { yes, no }\end{array}$ \\
\hline
\end{tabular}

Metabolic demand is strongly correlated with body mass in animals (Ehnes et al. 2014), which is directly related to several biological rates and processes such as predation and decomposition (Chapter 3). Furthermore, body mass affects species'́ vulnerability to disturbances and is thus related to extinction risk. For example, Barnes et al. (2014b) found that small-bodied dung beetles were most negatively affected by edge effects and matrix degradation, whereas Senior et al. (2012) found that larger-bodied carnivorous ants were most negatively affected by land-use conversion to oil-palm plantations. As such, we specified body mass as both an effect trait and a response trait. Using lengthmass regressions from the literature, we calculated body mass from individual body lengths, measured to an accuracy of $0.1 \mathrm{~mm}$ (Supplementary Table 3.1). 
Dispersal capacity gives an indication of a species' ability to escape adverse biotic and abiotic stressors. Furthermore, it delimits a species' ability to reach other resource patches in fragmented anthropogenic landscapes (Öckinger et al. 2010). We therefore used dispersal capacity as a response trait, providing information on the ability of a species to cope with land-use change. Dispersal capacity was approximated by allocating individuals into three groups according to whether they have wings, legs or both (Table 4.1). In cases where collected specimens were wingless juveniles but were known to have a winged life stage, we allocated these animals to a winged category.

Although the evolution of eusociality is relatively rare, where this trait has evolved, these species are often competitively superior within communities (Wilson \& Hölldobler 2005). As such, some eusocial species have been enormously successful, making them more resilient to environmental stressors and also allowing them to largely alter ecosystems through their acquisition of resources (Wilson \& Hölldobler 2005). Therefore, we included eusociality as both a response trait and an effect trait (Table 4.1). Specifically, eusociality was used as a categorical trait, with all ant and termite species coded as eusocial and all other species coded as non-eusocial.

Different strategies of energy uptake, such as predation, herbivory, decomposition or omnivory can have a strong influence on community energy fluxes and ecosystem functioning (Chapter 3). Species from different phylogenies within a trophic group, when taking into account body size, have also been shown to carry out highly similar ecosystem functions (Rall et al. 2011). Furthermore, feeding type is directly related to trophic position in food webs, which can therefore determine a species' sensitivity to environmental change. For example, predators located at the top of food webs depend on the stability of prey populations at lower trophic levels (Ryall \& Fahrig 2006). Because feeding type can determine species' effects on ecological processes, as well as species' sensitivity to environmental change, we specified feeding type as both a response and an effect trait (Table 4.1). The feeding type of each animal was classified based on a combination of information from literature, taxonomy and also morphology.

\subsubsection{Functional group richness and functional dispersion}

Using all measured functional traits, we quantified functional group richness and functional dispersion (Laliberté \& Legendre 2010) as measures of functional diversity. Although caution should be taken to use sufficient traits for accurately assessing functional diversity (Maire et al. 2015), given the broad taxonomic scale of our study, we 
incorporated as many broadly relevant traits as possible to maintain ecological realism. Firstly, all species were divided into functional effect groups (using only their effect traits) and again into functional response groups (using only their response traits) using the 'FD' package in R 3.0.3 (R Core Team 2014). To do this, we computed a Gower dissimilarity matrix from the trait values of all morphospecies and constructed a dendrogram that clusters species together based on their trait values (Laliberté et al. 2010). From the dendrogram, we chose a horizontal cut-off point to separate species into functional groups according to their trait combinations. Specifically, the chosen cut-off point avoided grouping of functionally dissimilar species such as predators and detritivores, but at the same time ensured that groupings were based on all traits measured (e.g., so that groups could also be defined by body size or eusociality) to generate ecologically relevant groups. This process yielded 18 functional response groups and 14 functional effect groups (Supplementary Table 4.1).

Secondly, using all measured traits and species composition at each site, we calculated functional dispersion (Laliberté \& Legendre 2010) as a measure of variation in community trait composition, using the 'FD' package in R (R Core Team 2014). Functional dispersion calculates the mean distance of species to the community traitmean centroid weighted by their relative abundances (Laliberté \& Legendre 2010). Because functional dispersion is a continuous measure of trait dispersion in multivariate trait space, it is insensitive to changes in species richness, rendering it an ideal metric to quantify convergence or divergence of communities in trait composition. We calculated functional dispersion using presence-absence data because rare species can have a significant influence on ecological processes, which could be masked in abundanceweighted calculations where rare species become less important (Laliberté et al. 2010).

\subsubsection{Calculating measures of community stability and resilience}

We quantified functional redundancy by calculating the mean number of species per functional group within each community. Here, we utilised only the functional effect groups (Supplementary Table 4.1), where species are grouped based on effect traits that give an indication of their functional roles. Generally, functional redundancy measures the vulnerability of communities to the loss of functional effect groups due to future ecological perturbations, thus providing a measure of functional stability (Laliberté $e t$ al. 2010). This approach assumes that species within a functional effect group all carry out a common ecosystem function, and so a loss of species does not necessarily lead to a 
complete loss of the shared function. Functional redundancy is a useful tool in ecology and conservation (Rosenfeld 2002), but its reliability depends on careful grouping and choice of traits. In this study, functional redundancy can be literally interpreted as the number of species that can be lost before losing an entire functional effect group, or ecosystem process.

To quantify response diversity, we calculated the mean functional dispersion of functional response groups of each sampled community, using presence-absence data within the 18 functional response groups (Supplementary Table 4.1). Response diversity is a measure of community resilience to future perturbations, specifically indicating the number of response traits present in a community that are adapted to dealing with various perturbations (Laliberté et al. 2010). For example, low response diversity indicates that all species within a community will have similar responses to ecological perturbations. Such a community may be resilient to one particular type of perturbation but highly susceptible to most others due to a lack of traits allowing species to respond differently (Elmqvist et al. 2003).

\subsubsection{Statistical analyses}

To assess our sampling effort of litter macro-invertebrate communities, we calculated sample-based species accumulation curves in EstimateS 9 (Colwell 2013). Mao Tao estimates of species richness within each of the four land-use systems were calculated from 100 random draws without replacement. Additionally, we used the second order jacknife estimator (Brose et al. 2003) to calculate extrapolated species richness from the three $1 \mathrm{~m}^{2}$ subplots at each of the 32 sites, with the 'vegan' package in R (R Core Team 2014). We then estimated sampling coverage by calculating the proportion of observed out of total (extrapolated) species richness at each site (see Brose et al., 2003 for evaluation of this procedure). These analyses revealed that we achieved sampling completeness between 50 and $60 \%$ across the sampling sites with very even sampling coverage among land-use systems (see Supplementary Figure 4.1). Although we feel that this allows for reasonably reliable comparisons of taxonomic and functional diversity among these systems, our results should be taken with some caution due to potential under-sampling of rare species with variable traits.

Using linear mixed effects models, we tested the effect of land-use system on species richness, animal density, functional group richness (of response groups), functional dispersion, functional redundancy and response diversity with 'landscape' (i.e., 
Bukit Duabelas and Harapan landscapes) specified as a random effect. Tukey post hoc tests were applied with a Bonferroni correction to test for significant differences among land-use systems. To meet the assumptions of normality, density, functional group richness and response diversity were $\log _{10}$-transformed and modelled on a normal distribution. Species richness, which was overdispersed Poisson-distributed data, was modelled on a negative-binomial distribution. Furthermore, to determine whether our analyses might be affected by spatial autocorrelation, we calculated Moran's I values for each model's residuals. We then tested for spatial autocorrelation using the Moran's I standard deviate (Dormann et al. 2007) in the 'spdep' package in R (R Core Team 2014) and found no significant effect of spatial autocorrelation in our models (lowest Moran's I test result yielded $\mathrm{p}=0.391$ ).

We applied a multivariate approach to test the relationship between relative abundances of the functional response groups and land-use change, allowing us to further assess functional trait-based community assembly processes and stability. Firstly, we calculated a dissimilarity matrix using the 'modified Gower' dissimilarity metric (Anderson et al. 2011) and then visualized the relationship between land-use change and relative abundances of functional response groups using a nonmetric multidimensional scaling ordination (NMDS). We then tested if the dissimilarity among communities based on functional response group relative abundances was dependent on the land-use system by performing a permutational multivariate analysis of variance (permutational MANOVA) test with 1000 permutations. Finally, we used the 'betadisper' function to test for differences in multivariate dispersion of communities in their functional response group composition among land-use systems. All statistical analyses were conducted in $\mathrm{R}$ (R Core Team 2014) using the 'nlme', 'lme4', 'multcomp', and 'vegan' packages.

\subsection{RESULTS}

\subsubsection{Species richness and animal densities}

A total of 7544 animals from 896 morphospecies were collected across the 32 sampling sites in four different land-use systems (Supplementary Table 4.2). The most abundant taxonomic group was Hymenoptera ( $40 \%$ of all individuals), clearly dominated by ants (Formicidae), and followed by Araneae (14\%), Isoptera (10\%), Psocoptera (7\%) and Coleoptera (6\%). Species were grouped into 14 functional effect groups and 18 functional 
response groups across all sites (Supplementary Table 4.1). Land-use change had a negative impact on both species richness and animal density of macro invertebrates, with a $46 \%$ decrease in species richness $(\mathrm{t}=-3.96, \mathrm{df}=26, \mathrm{p}<0.001)$ from forest systems to oil palm (Figure 4.2a). Specifically, the mean number of species decreased from 96 ( \pm $7.88 \mathrm{SE})$ species in forest systems to $52( \pm 7.82 \mathrm{SE})$ species in oil-palm systems (Figure 4.2a). Densities of litter macro-invertebrates were also significantly impacted, with a $48 \%$ reduction from forest to oil-palm systems $\left(\mathrm{F}_{3,27}=3.393, \mathrm{p}=0.032\right.$, Figure $\left.4.2 \mathrm{~b}\right)$.

a)

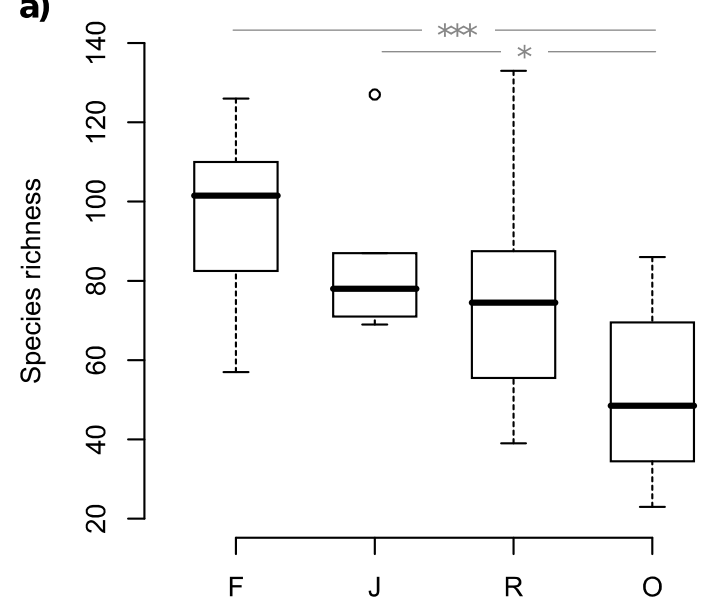

c)

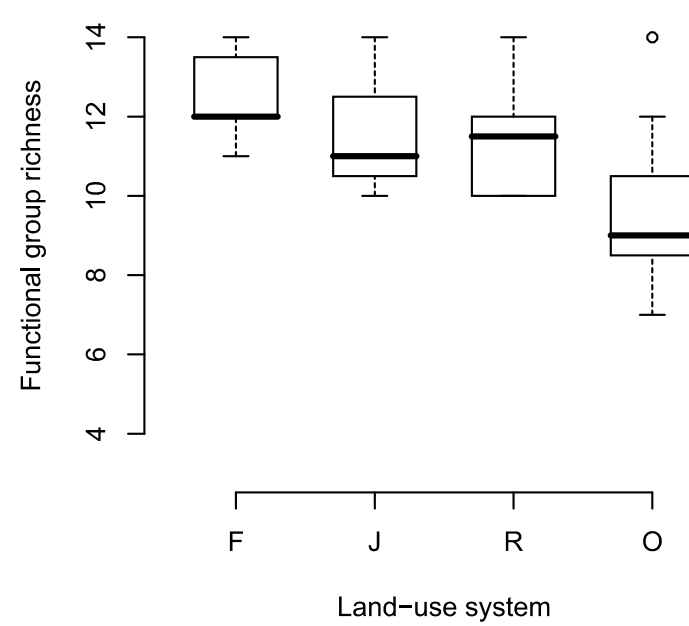

b)

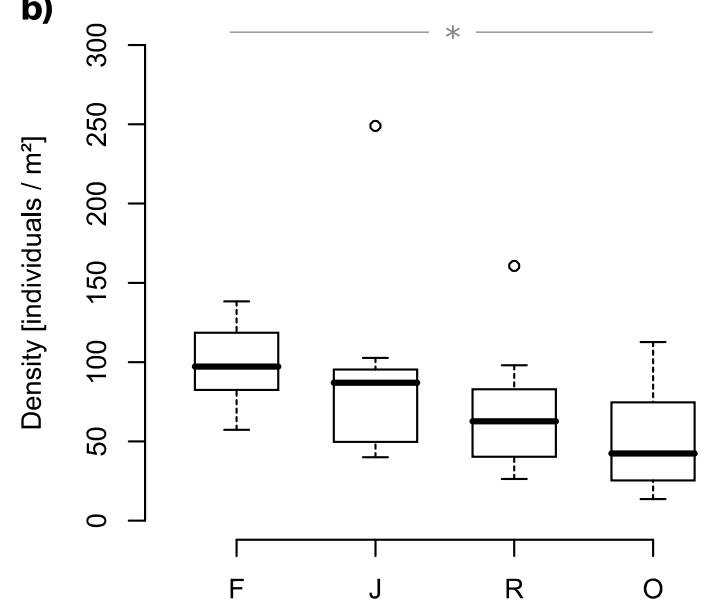

d)

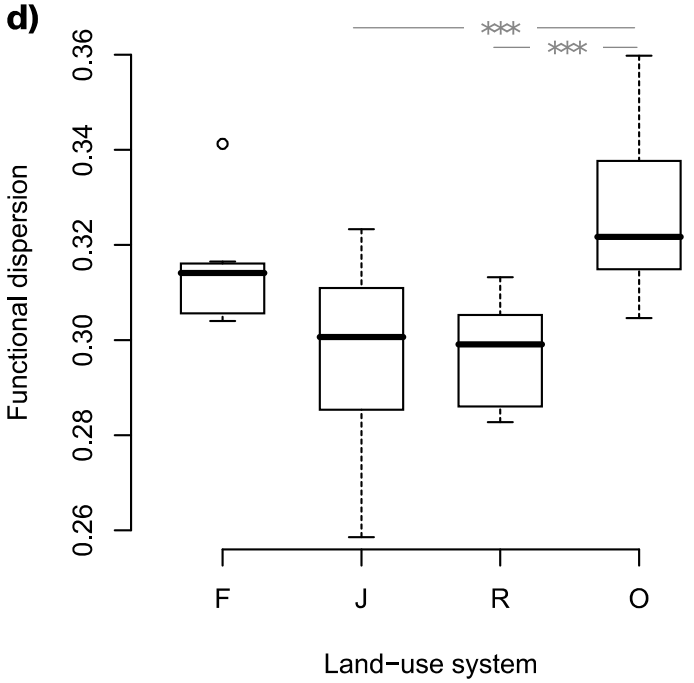

Figure 4.2: Macro-invertebrate community responses to land-use change. Species richness (a), animal density (b), functional group richness (c) and functional dispersion (d) for the four landuse systems $(\mathrm{n}=32)$. Asterisks denote significance levels: $* \mathrm{p}<0.05 ; * * \mathrm{p}<0.01 ; * * * \mathrm{p}<0.001$. Boxes indicate the first and third quartiles, with the median and $95 \%$ confidence intervals denoted by the central line and whiskers, respectively, and outliers indicated by open symbols. 


\subsubsection{Responses in functional diversity}

Richness of functional response groups showed a significant negative response to landuse change $\left(\mathrm{F}_{3,27}=11.447, \mathrm{p}<0.001\right)$ from forest to the more intensively managed systems like rubber and oil-palm plantations, although the magnitude of the decrease was not as dramatic as that found in species richness (23\% compared to $46 \%$ loss). The number of functional groups decreased from a mean of $13( \pm 0.378 \mathrm{SE})$ to $10( \pm 0.800$ $\mathrm{SE}$ ) of the total 18 functional response groups from forest to oil-palm communities (Figure 4.2c), where we consistently detected no occurrence of functional groups 6 and 15 (Supplementary Figure 4.2). Interestingly, the functional response group of winged predators (functional response group 9, Supplementary Table 4.1), including animals such as predatory beetles, exhibited the strongest decrease in density from forest to oil-palm systems, with only $12 \%$ of individuals from this functional response group remaining. The second strongest decrease in density (22\% remaining in oil palm) was found within the functional response group of non-winged predators with high body mass, mainly comprised of large-bodied spiders (functional response group 3, Supplementary Table 4.1), and the third strongest decrease was found for non-winged omnivores (26 remaining in oil palm), including animals such as Gryllidae crickets (functional response group 4, Supplementary Table 4.1) (see Supplementary Figure 4.2 for density responses of all functional response groups).

Despite the clear decrease in species diversity, we found similar levels of functional dispersion in forest and the two rubber systems, compared to much higher values in oil-palm plantations $\left(\mathrm{F}_{3,27}=6.972, \mathrm{p}=0.001\right)$ (Figure 4.2d). Specifically, the Tukey post hoc test suggested that functional dispersion in oil palm was significantly higher than in rubber $(\mathrm{p}<0.001)$ and jungle rubber $(\mathrm{p}<0.001)$ (Figure 4.2d), indicating that functional heterogeneity is higher in oil palm than in the other land-use systems (but not significantly higher than in forest).

Because of the observed losses in animal density and species richness, we suspected that relative abundances of functional response groups would be altered among land-use systems. Results from the permutational MANOVA suggested that land-use change explained a significant amount of variation in the compositional dissimilarity of communities based on relative abundances of functional response groups ( $p<0.001)$. These differences were evident in the NMDS plot of community relative abundances of functional response groups among the land-use systems (Figure 4.3). In particular, the NMDS showed a clear separation of oil-palm communities from all other land-use 
systems, visualized by no overlap of the $95 \%$ confidence interval with any of the other land-use systems. Furthermore, forest systems were more similar in functional group composition to rubber and jungle-rubber plantations than to oil-palm systems (Figure 4.3). Interestingly, we found the highest multivariate dispersion of communities within the oil-palm sites, which were significantly higher than in both jungle-rubber and forest systems $\left(F_{3,27}=5.777, p=0.003\right)$. This indicates that the communities of different oilpalm plantations are less similar to each other in functional composition.

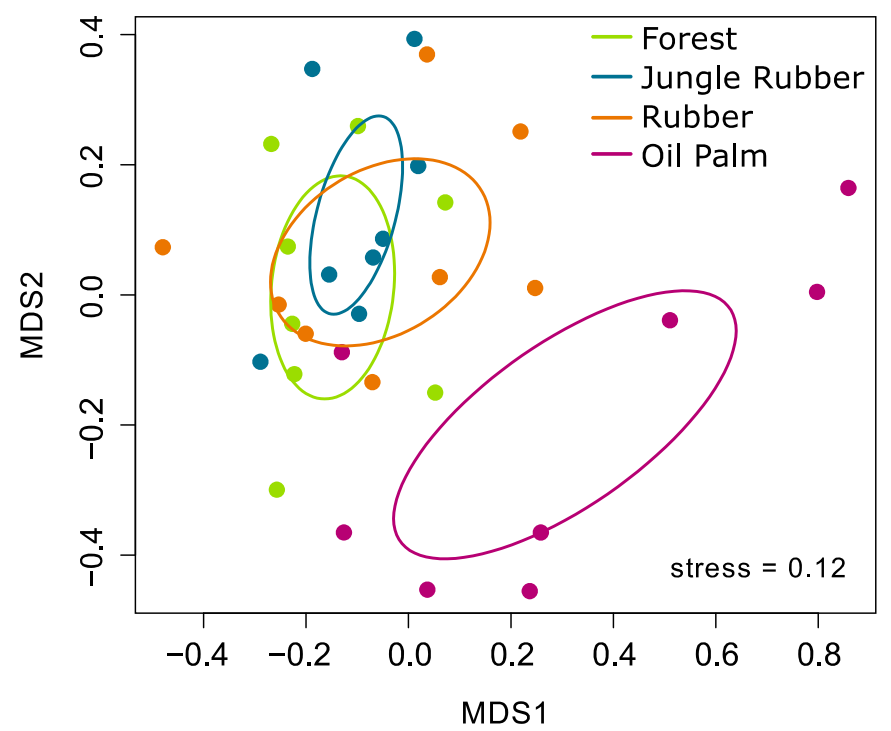

Figure 4.3: Functional trait composition of macro-invertebrate communities. NMDS ordination plot showing the compositional dissimilarity of litter communities among the four land-use systems based on the relative abundances of functional response groups. Each point represents one of the 32 sampled communities, with $95 \%$ confidence ellipses for each of the land-use systems.

\subsubsection{Functional redundancy and response diversity}

Similar to patterns in species diversity, we found a strong decrease in functional redundancy from forest to the more intensively managed land-use systems. From forest to oil-palm systems, an average of $37 \%$ of functionally redundant species within the functional effect groups were lost $\left(\mathrm{F}_{3,27}=3.073, \mathrm{p}=0.045\right.$, Figure $\left.4.4 \mathrm{a}\right)$. In particular, we found that litter-invertebrate communities in oil-palm plantations had significantly lower functional redundancy than in primary degraded forest $(\mathrm{p}=0.031)$. Hence, species loss following land-use change to oil-palm occurred throughout all of the functional effect groups, resulting in fewer species within each functional effect group and thus rendering 
these communities more susceptible to complete losses of whole ecosystem functions. Despite the fact that we found clear responses in functional diversity and functional redundancy, response diversity yielded no significant response to land-use change $\left(\mathrm{F}_{3,27}\right.$ $=2.778, \mathrm{p}=0.060$ ) (Figure 4.4b). However, there did appear to be a weak trend of decreasing response diversity from forest to oil-palm systems (but higher values in monoculture rubber), but this trend was disrupted with very high variability within the different land-use systems.
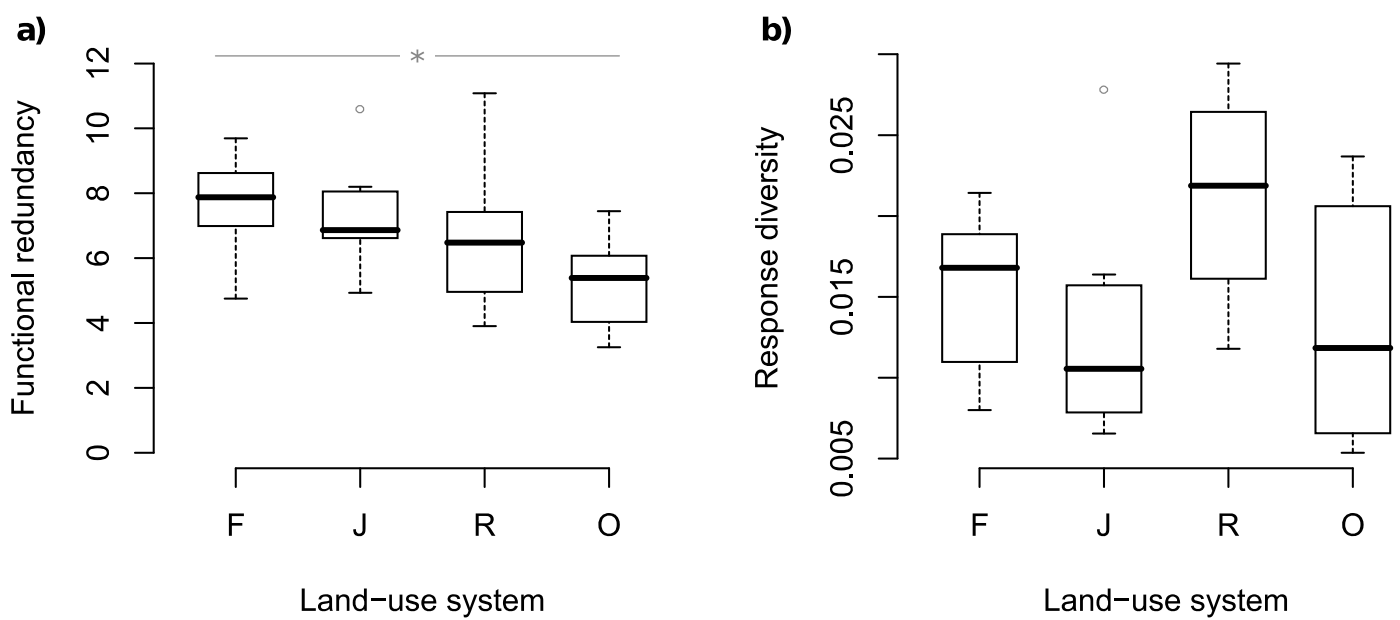

Figure 4.4: Functional stability and resilience of macro-invertebrate communities. Functional redundancy (number of species within functional effect groups) (a) and response diversity (functional dispersion for each functional response group) (b) for the four land-use systems $(\mathrm{n}=32)$. Asterisks denote significance levels: $* \mathrm{p}<0.05 ; * * \mathrm{p}<0.01 ; * * *<0.001$. Boxes indicate the first and third quartiles, with the median and $95 \%$ confidence intervals denoted by the central line and whiskers, respectively, and outliers indicated by open symbols.

\subsection{DISCUSSION}

The global trend of increasing land-use intensification has led to widespread negative impacts on both taxonomic and functional diversity (Flynn et al. 2009), resulting in significant losses in ecosystem functioning and increased ecological uncertainty (Laliberté et al. 2010; Cadotte et al. 2011). In this study, we found that the conversion of tropical rainforest to agroforests and monoculture plantations can lead to important shifts in functional trait composition of the highly diverse invertebrate communities in these systems. Specifically, this was demonstrated by the combination of decreased species and functional group richness, along with increased functional dispersion and functional 
response group-based community dissimilarity in oil-palm plantations compared to less intensively managed systems. These changes in litter-invertebrate community trait composition, along with species losses, resulted in a significant decrease in functional redundancy within functional effect groups. In contrast, response diversity was not significantly altered by land-use change. Taken together, our results suggest that the conversion of tropical rainforest to intensively managed plantation agriculture can strongly alter the functional composition of tropical leaf-litter communities, likely threatening the functional stability of these systems.

\subsubsection{Effect of land-use change on species diversity and animal density}

We found a significant decrease from forest to oil-palm communities in species richness (46\%) and animal density (48\%), supporting previous findings that land-use change imposes clear negative effects on invertebrate communities (Jones et al. 2003; Eggleton et al. 2005). Because leaf-litter-dwelling macro-invertebrates are highly dependent on litter input from vegetation, reduction in plant diversity and biomass resulting from landuse conversion into intensively managed monoculture plantations (such as rubber and oil palm in our study) most likely plays a major role in altering species diversity and densities of litter macro-invertebrates (Gillison et al. 2003). Specifically, the reduced stoichiometric diversity of leaves in the litter layer of monocultures may play a pivotal role in affecting the density and diversity of the litter-animal communities (Kaspari \& Yanoviak 2009; Ott et al. 2014b). Additionally, the litter layer depth was lower in the oilpalm and rubber monocultures than in the more diverse forest and jungle-rubber systems (Kotowska et al., unpublished data), which can strongly reduce the diversity of litter communities (Klarner et al. 2014; Digel et al. 2014). Interestingly, jungle rubber, which harbours higher plant species richness (Gouyon et al. 1993) and thus tends to have a consistently thicker and more species-rich litter layer (Kotowska et al., unpublished data), also had significantly higher macro-invertebrate species richness than oil-palm systems, indicating its potential conservation value for these functionally important communities.

\subsubsection{Land-use change alters functional diversity of litter macro-invertebrates}

We detected a significant reduction of functional response group richness, with three less functional response groups, on average, in litter-invertebrate communities sampled in oilpalm plantations compared to forest systems. In particular, the groups that appeared to be consistently absent from the oil-palm plantation sites tended to be those with already very 
low numbers of individuals and species (i.e., functional response groups 6 and 15; Supplementary Table 4.1). These findings indicate that where functional redundancy is low, land-use conversion could result in losses of whole functional groups and this could lead to eventual losses of associated ecosystem functions. That being said, such conclusions should be taken with caution due to rather limited sampling completeness (see Supplementary Figure 4.1), which could have resulted in the underrepresentation of rarer species that might be important for retaining these missing functional groups. Results from the test of multivariate dispersion in the NMDS ordination of functional response group composition revealed that there was significantly higher functional dissimilarity of invertebrate communities in oil-palm plantations, lower in rubber and the lowest in jungle-rubber plantations. Thus, litter communities in oil-palm plantations were more dissimilar to each other in their functional composition than those within the other less intensively managed land-use systems. This could mean that communities are more randomly assembled in the more highly disturbed oil-palm and rubber plantations than in jungle-rubber plantations and forest. Nevertheless, results from the permutational MANOVA on the relative abundances of functional response groups revealed that there are still consistent differences among the land-use types in functional group composition. These results suggest that from forest to oil-palm systems there may be a non-random, trait-dependent loss of individuals from particular functional response groups.

Interestingly, we found significantly higher functional dispersion in oil-palm systems than in all other land-use systems. Prior studies have found low-intensity landuse systems with lower functional dispersion (Luck, Carter \& Smallbone 2013) or no significant impact of land-use change on functional dispersion (Barragán et al. 2011; Audino, Louzada \& Comita 2014). Here, the higher functional dispersion found in oilpalm communities suggests that they are subjected to more diffuse ecological filtering resulting in more randomly assembled macro-invertebrate communities. With oil-palm plantations normally being re-established after a period of about 25 to 30 years (Fitzherbert et al. 2008), along with very regular clearing of leaf litter and also regular application of pesticides (Foster et al. 2011), there is a high frequency of severe disturbances that would prevent the gradual convergence of traits in communities assembling in these habitats (Fukami et al. 2005). Rather, it is likely that species occurring in such highly disturbed oil-palm systems are generalists that randomly disperse into these habitats, allowing them to persist in conditions where resources are spatially and temporally unstable (Devictor, Julliard \& Jiguet 2008). Taken together, 
these results are concerning for the conservation of ecosystem functioning in anthropogenic landscapes as they suggest that the provisioning of ecosystem processes is likely to be unstable in intensively managed plantation agriculture, such as oil palm (Edwards et al. 2014a).

\subsubsection{Functional redundancy and response diversity under land-use intensification}

The maintenance of functional redundancy in ecological communities is crucial for the stability of ecosystem processes (Flynn et al. 2009). Despite its importance, the impact of land-use change on functional redundancy has not yet been explored in highly diverse animal communities spanning multiple higher-level taxonomic groups. We found an average loss of $37 \%$ of functionally redundant litter macro-invertebrate species from forest to oil palm. As such, the risk of losing whole ecosystem functions clearly increases with conversion of natural ecosystems to plantation agriculture, thus critically reducing the functional stability of these altered ecosystems (Fonseca \& Ganade 2001; Pillar et al. 2013). For example, we did not detect any large-bodied detritivores in these monoculture systems; given further perturbations, which are becoming increasingly likely with ongoing climate change (Jentsch, A., Kreyling, J., \& Beierkuhnlein 2007), this may be a real concern for other groups that also had strongly decreasing redundancy. As a result, the facilitation of litter decomposition could be largely reduced where these functional effect groups are lost (Lang et al. 2014). Because our measure of functional redundancy does not allow for overlap of functions among different functional effect groups (i.e., omnivores are not mixed with other feeding types within functional effect groups), these results should also be interpreted with some caution. Nevertheless, the magnitude of response in functional redundancy that we detected indicates that it is unlikely such losses could be completely buffered by other generalist omnivore species, still highlighting reasonable concern for the functional stability of these altered systems.

Interestingly, despite the disparity in functional redundancy between the forest and oil-palm systems, there was no significant difference in functional redundancy between the two rubber land-use systems and the forest. This suggests that low-intensity agroforestry systems such as jungle rubber could serve as reservoirs of functional diversity and stability in tropical anthropogenic landscapes (Edwards et al. 2014b), where species could spill over into higher-intensity agricultural systems (Lucey \& Hill 2012). Therefore, interspersing low-intensity agroforestry systems like jungle rubber throughout 
oil-palm dominated landscapes might effectively maintain functional diversity and stability of ecosystem functioning in these increasingly homogenous landscapes.

Contrary to the strong responses of functional redundancy to land-use change, we did not detect any effect of land-use change on response diversity. It is possible that the disturbance intensity and frequency in the monoculture plantation systems is so high that there is very diffuse ecological filtering and, thus, a random loss of species. In such a case, this should yield similar levels of diversity in response traits across land-use systems. These processes could explain why we found only weak effects of land-use intensification on the response diversity of these tropical macro-invertebrate communities.

\subsubsection{Conclusions}

In this study, we demonstrate for the first time that tropical land-use change from forest to intensively-managed plantation agriculture could alter the functional stability of highly diverse invertebrate communities by impacting their functional trait composition. In general, our results suggest that litter dwelling macro-invertebrate communities tend to be more randomly assembled and compositionally unstable in intensively managed systems such as oil-palm plantations. Ultimately, these findings suggest that communities within intensively managed tropical plantations are highly susceptible to losing entire ecosystem functions - such as the facilitation of decomposition rates by macro-invertebrate detritivores or the suppression of invertebrate pests by larger invertebrate predatorsgiven future ecological perturbations, which puts these systems at risk of losing crucial ecosystem services. With the worrying future scenarios of climate change and continued agricultural intensification in Southeast Asia, this presents a real concern for the future functional stability of tropical agricultural ecosystems. Despite these concerning results, our study also provides insight into the potential for intermediate intensification levels of plantation agriculture, such as jungle-rubber plantations, to maintain relatively higher levels of taxonomic diversity, functional diversity, and functional stability in anthropogenic production landscapes. 


\subsection{ACKNOWLEDGEMENTS}

We thank Megawati, Rizky Nazarreta, Keisha Disa Putirama, Rosario Reza Valentino Lasse for assistance in the field and laboratory; Martyna Kotowska at the Plant Ecology \& Ecosystem Research Department, University of Göttingen, for providing litter-fall data; and two anonymous reviewers for helping to greatly improve earlier versions of this manuscript. We also thank the village leaders, local site owners, PT REKI, and Bukit Duabelas National Park for granting us access to their properties. This study was financed by the Deutsche Forschungsgemeinschaft (DFG) in the framework of the collaborative German - Indonesian research project CRC990. 
Chapter 5

\section{Spatial Turnover in Biodiversity and Ecosystem Functioning Across Tropical and Temperate Ecosystems}

Andrew D. Barnes, Patrick Weigelt, Malte Jochum, David Ott, Dorothee Hodapp, Noor Farikhah Haneda, and Ulrich Brose

\subsection{ABSTRACT}

Predicting ecosystem functioning at large spatial scales rests on our ability to scale up from local plots to landscapes, but this is highly contingent on our understanding of how functioning varies through space. Such an understanding has been hampered by a strong experimental focus of biodiversity-ecosystem functioning research restricted to small spatial scales. To address this limitation, we investigate the drivers of spatial variation in multitrophic energy flux - a measure of ecosystem functioning in complex communities - at the landscape scale. We use a structural equation modelling framework based on distance matrices to test how spatial and environmental distances drive variation in community energy flux via four mechanisms: species composition, species richness, niche complementarity, and biomass. We found that in both a tropical and temperate study region, geographic and environmental distance indirectly influence species richness and biomass, with clear evidence that these are the dominant mechanisms explaining variability in community energy flux over spatial and environmental gradients. Our results reveal that species composition and trait variability may become redundant in predicting ecosystem functioning at the landscape scale. Instead, we demonstrate that species richness and total biomass may best predict rates of ecosystem functioning at larger spatial scales. 


\subsection{INTRODUCTION}

Anthropogenic alteration of the Earth's ecosystems is so immense that scientists have suggested the commencement of a new geological epoch, the Anthropocene (Zalasiewicz et al. 2011). One of the most notable impacts of human activities has been the global reduction in biodiversity (Pimm et al. 2014; Ceballos et al. 2015), stimulating widespread concern over the resulting loss of ecosystem functioning and services provided by natural systems (Díaz et al. 2006; Cardinale et al. 2012). In the past two decades, considerable effort has been made to understand the mechanisms that drive rates in ecosystem functioning, with an especially large focus on the importance of biodiversity (Balvanera et al. 2006; Cardinale et al. 2012; Tilman et al. 2014). Most of this research has emerged from experimental studies that attempt to directly link species richness with ecosystem processes, such as biomass production. In more recent years, attention has also shifted toward the importance of functional diversity (the diversity of functional traits) and phylogenetic diversity in driving ecosystem functioning (Reiss et al. 2009; Flynn et al. 2011). However, due to the largely experimental nature of the research that has developed in this field so far, little is understood about patterns in ecosystem functioning at the landscape scale and the mechanisms driving these patterns (Cardinale et al. 2012; Tilman et al. 2014).

The importance of spatial context in biodiversity and ecosystem functioning (BEF) research has been increasingly realised in recent years (Chalcraft et al. 2004; Godbold, Bulling \& Solan 2011). For example, France \& Duffy (2006) demonstrated that metacommunity structure and dispersal were highly important for maintaining rates and temporal stability of productivity. This study focused primarily on the role of species richness for determining rates and stability of biomass production in a relatively simple system limited to two trophic levels (primary producers and grazers) and up to nine species in a given treatment. In comparison, forest soil ecosystems are globally widespread and harbour remarkably high numbers of species that span many trophic levels and yield highly complex food webs (e.g., Digel et al. 2014) that are directly related to important ecosystem services (Handa et al. 2014). This raises the question of how ecosystem functioning of multitrophic communities varies across space in terrestrial forest ecosystems where species turnover is relatively high (Basset et al. 2012). Incorporating such high levels of diversity is challenging, if not impossible, for manipulative BEF experiments, thus calling for the implementation of landscape-level 
field research that extends beyond correlative analyses and rather tries to identify causal mechanisms (Cardinale et al. 2012; Tilman et al. 2014). A major hindrance to the implementation of this approach has been the difficulty of directly linking measured ecosystem functions (such as decomposition, predation and herbivory rates) with multitrophic species assemblages that are sampled at the landscape level. In this study, we overcome this limitation by analytically calculating energy fluxes among biomass pools via trophic interactions in natural litter macroinvertebrate communities.

Using calculated multitrophic energy flux, we address four hypotheses of how ecosystem functioning can vary over spatial and environmental gradients. Firstly, the composition of species might affect energy fluxes due to particular attributes of species that confer especially important effects on resource uptake (Hooper et al. 2005) (Figure1, H1). Thus, turnover of species among communities ( $\beta$-diversity) should scale positively with dissimilarity in community energy fluxes (Figure 5.1, H1). Secondly, community energy flux might scale with species richness ( $\alpha$-diversity) due to concurrent variability in resource heterogeneity (Figure 5.1, H2) (Tylianakis et al. 2008b). This would occur as the addition of resource species allows consumers to increase their resource uptake because of an increase in available resource niches. In this case, we would expect that dissimilarity in $\alpha$-diversity should be positively correlated with dissimilarity of community energy fluxes (Figure 5.1, H2). Thirdly, we hypothesize that community energy fluxes scale positively with functional diversity (hereafter functional dispersion) because a wider range of traits should allow consumers to feed on a wider range of resource species. Specifically, given sufficient resource heterogeneity (Tylianakis et al. 2008b), a larger range of traits among consumers should reduce competition over shared resources due to increased resource partitioning in more functionally diverse consumer assemblages, allowing for the complementary use of resources (Figure 5.1, H3) (Striebel et al. 2009; Poisot, Mouquet \& Gravel 2013; Gagic et al. 2015). Therefore, if niche complementarity determines rates of functioning, we would expect that dissimilarity of functional dispersion should scale positively with dissimilarity of community energy fluxes (Figure 5.1, H3). Finally, rates of energy flux may be purely biomass dependent, regardless of the species assemblage, because greater species biomass inherently increases energetic demands and, thus, energy flux among biomass pools. In such a case, we would expect a positive relationship between dissimilarity in community biomass and community energy fluxes (Figure 5.1, H4). 

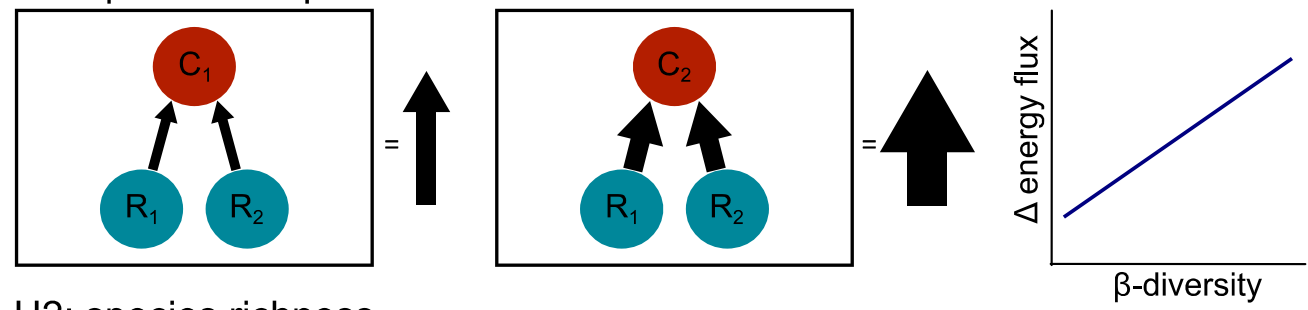

H2: species richness
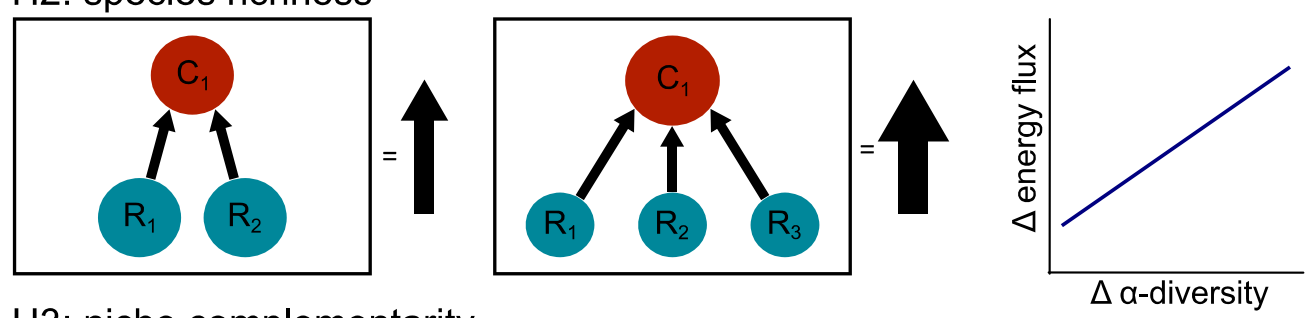

H3: niche complementarity
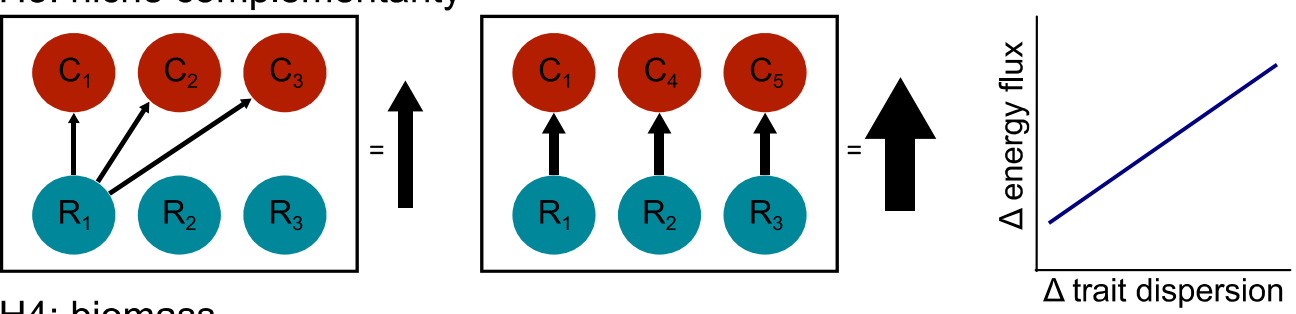

H4: biomass

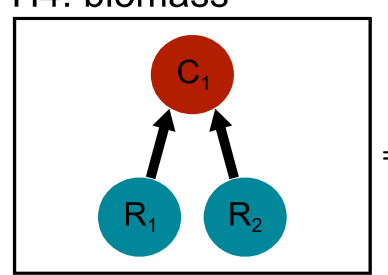

Plot A

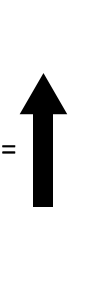

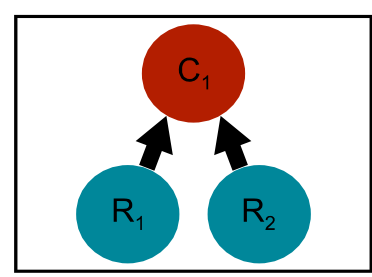

Plot B
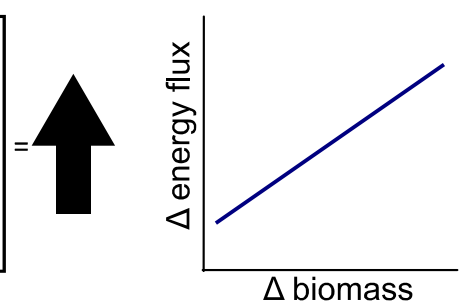

Geographic and environmental gradient

Figure 5.1: Graphical representation of the four proposed mechanisms $(\mathrm{H} 1-\mathrm{H} 4)$ that drive spatial variation in ecosystem functioning. Hypothetical examples illustrate how each mechanism would drive variation in energy flux (black arrows) between two spatially and environmentally distinct sampling plots (plot A and B). Blue and red circles represent biomass-weighted (circle size) resource and consumer populations, respectively, with $\mathrm{R}_{\mathrm{i}}$ and $\mathrm{C}_{\mathrm{i}}$ denoting different resource and consumer species. Graphs to the right of the example motifs indicate the hypothesized relationship between turnover in the measured community attribute used to test the hypothesis and turnover in energy flux.

In this study, we disentangle the relative contributions of $\beta$-diversity, $\alpha$-diversity, functional dispersion, and total biomass on community energy fluxes in temperate and tropical litter macroinvertebrate communities. Using standardized sampling, we compared litter invertebrate communities across two extensive spatial and environmental gradients in managed German forests (48 plots) and plantation agriculture and agroforests in Sumatra, Indonesia (32 plots) to assess the relative roles of spatial and environmental 
distance in determining community assembly and how these processes give rise to variation in ecosystem functioning. In doing so, we disentangle the mechanisms that mediate variation in ecosystem functioning across landscapes and determine how geographic distance can be used to predict ecosystem functioning at large spatial scales.

\subsection{METHODS}

\subsubsection{Study sites}

To disentangle the mechanisms responsible for driving variation in ecosystem functioning at the landscape scale, we compared plots across two spatially extensive study regions comprising two landscapes in Sumatra, Indonesia and three landscapes in Germany (Supplementary Figure 5.1). Plots in both regions were established across a range of land-use management intensities. In Indonesia, plots were located in each of four land-use types: primary degraded forest, jungle rubber agroforests, monoculture rubber, and monoculture oil palm, replicated four times in each of two spatially independent landscapes located near Bukit Duabelas National Park and Harapan Rainforest, making a total of 32 plots. In Germany, plots were located in each of four land-use types of varying management intensity: unmanaged beech forest, 70 year-old managed beech forest, 30 year-old managed beech forest, and intensively managed coniferous forest. These plots were replicated four times in each of three spatially independent landscapes in the Swabian Alb Biosphere Reserve, the Hainich National Park and the Schorfheide-Chorin Biosphere Reserve, making a total of 48 plots (see Ott et al. [2014] for details of study design). Within each region, the plots covered a large geographical range, with inter-plot distance ranging from 0.1 to $90 \mathrm{~km}$ in Indonesia and from 0.3 to $630 \mathrm{~km}$ in Germany.

\subsubsection{Quantifying spatial and environmental distance}

Pairwise spatial distances among plots were calculated as Great Circle distances in $\mathrm{km}$ separately for each study region. To quantify environmental distance, a total of 15 measured environmental parameters were used to characterise the 80 plots across both study regions: mean soil moisture content, mean soil temperature, soil $\mathrm{pH}$, litter depth, and 11 different elements measured from the leaf litter $(\mathrm{C}, \mathrm{N}, \mathrm{P}, \mathrm{Al}, \mathrm{Ca}, \mathrm{Fe}, \mathrm{K}, \mathrm{Mg}, \mathrm{Mn}$, $\mathrm{Na}$, and S). Soil moisture content $(\%)$ and soil temperature $\left({ }^{\circ} \mathrm{C}\right)$ were recorded hourly using soil sensors placed at $30 \mathrm{~cm}$ depth in the soil within each plot. Soil $\mathrm{pH}$ was analysed 
in a 1:4 soil-to-water ratio at the Indonesian plots (Allen et al. 2015) and a 1:10 soil-tosolution $(\mathrm{CaCl})$ ratio for the German plots (Ott et al. 2014a). Leaf litter samples were collected at each of the 80 research plots and the amounts $(\mathrm{mg})$ of 11 different elements in leaf litter dry mass were analysed (see (Ott et al. 2014a) for details).

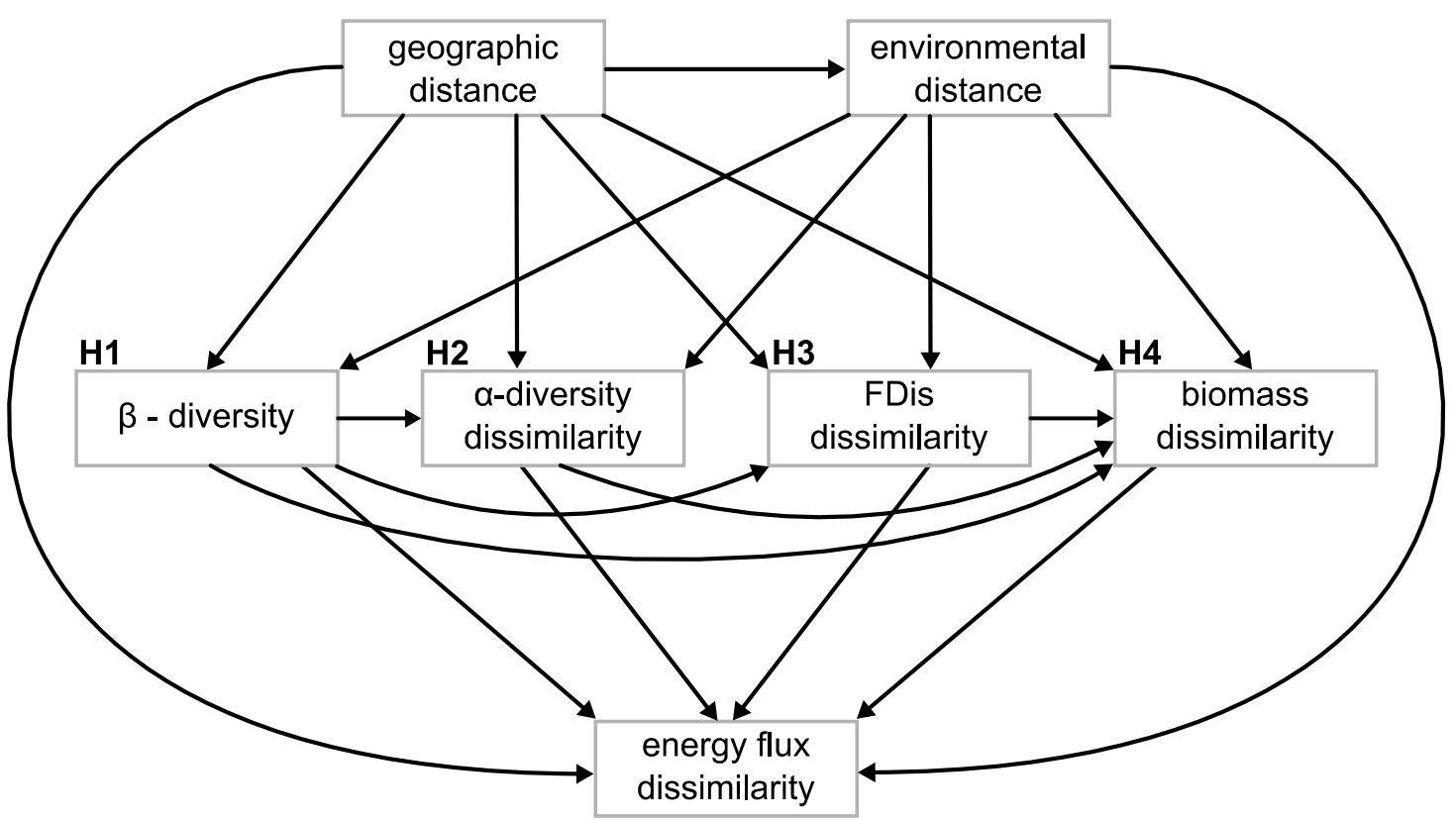

Figure 5.2: Diagram of all possible effects that were tested in the path model framework of this study. Variables in the path models are indicated by grey boxes and represent distance matrices. H1 - H4 denote where hypotheses 1 - 4 (as in Figure 5.1) were tested in the model. 'FDis' stands for functional dispersion.

To select the environmental parameters that are most important for explaining variation in biodiversity and ecosystem functioning of the sampled litter macroinvertebrate communities, we employed two steps. Firstly, we ran a non-metric multidimensional scaling (NMDS) ordination based on Jaccard dissimilarities of all macroinvertebrate communities. Using the 'envfit' function in the 'vegan' package in $\mathrm{R}$ (R Core Team 2015), we then performed a permutational vector fitting analysis of all 15 environmental parameters as raw and additionally log-transformed variables in the ordination (for both the temperate and tropical data). Only the vectors that yielded $\alpha<$ 0.05 from the permutation tests were retained and then standardized by subtracting their means and dividing by their standard deviations. In cases where both the logged and untransformed variables were significant, we selected the variable with the highest $R^{2}$ value (see Supplementary Table 5.1 for details). Finally, we ran a principle component analysis (PCA) with these retained variables, selected the site scores of the first three 
principal components $(74.75 \%$ and $78.11 \%$ variation contained in the first three axes for the Indonesian and German data, respectively) and calculated dissimilarities among all plots as Euclidean distances.

\subsubsection{Animal sampling and measurements}

In both regions, macroinvertebrates were sampled from the leaf litter using litter sieves. In Indonesia a total of three $1 \mathrm{~m}^{2}$ subsamples were taken at each plot between October and November 2012, and in Germany a total of four $0.25 \mathrm{~m}^{2}$ subsamples were taken from each plot in spring 2011. Leaf litter from each subsample was removed from the surface of the ground down to the soil and placed in a coarse mesh sieve. All visible invertebrates were hand collected and stored in ethanol, after which they were identified to species or morphospecies. The number of species per plot was recorded and the dissimilarity among plots in species richness ( $\alpha$-diversity) was then calculated as the log response ratio (LRR) between values, from which we compiled a dissimilarity matrix for each region. Furthermore, we calculated Jaccard dissimilarities among plots and compiled dissimilarity matrices from these values to quantify dissimilarity in species composition across each region.

Specimens were assigned to trophic groups based on a combination of taxonomy, morphology and information from literature. The body lengths $(\mathrm{mm})$ of all collected individuals were measured and then converted to live body masses (mg) using allometric equations from the literature (see Supplementary Table 3.1 for details and sources of allometric equations). In addition, we assessed mobility of collected specimens based on whether the individual was winged, legged, or both. If the specimen was a wingless juvenile but was known to have a winged life stage then it was allocated to a winged category. We also recorded whether or not each specimen was eusocial based on taxonomy. Further details describing the justifications for trait selection and their assignment can be found in Chapter 4. Finally, individual metabolic rates were calculated for all collected individuals with regression equations using body masses, temperatures measured at each plot, and taxonomic group (Ehnes et al. 2011).

Total community biomass was calculated by summing body masses from all individuals collected at each plot. We then compiled dissimilarity matrices based on LRR's of total biomass values among all plots in each region. As a way of quantifying heterogeneity in the functional roles of individuals present in each plot (i.e., to estimate the potential for niche complementarity in sampled communities), we calculated 
functional dispersion of communities (Laliberté \& Legendre 2010; Gagic et al. 2015) from four measured traits: trophic group, body mass, mobility, and eusociality. Specifically, functional dispersion calculates the mean distance of species to the community trait-mean centroid weighted by their relative abundances (Laliberté \& Legendre 2010). Functional dispersion was calculated using the 'FD' package in $\mathrm{R}$ ( $\mathrm{R}$ Core Team 2015). Dissimilarity matrices were also compiled from LRR's between functional dispersion values at each plot for each region.

To measure ecosystem functioning in a way that incorporates all sampled trophic levels into a single variable and can be easily quantified across large spatial scales, we analytically assessed community energy flux for all 80 sampled communities across both regions. To do so, we used the formula

$$
F=\frac{1}{e_{a}} \cdot(X+L)
$$

where $F$ is the total flux of energy into the biomass pool of a given trophic level, $e_{a}$ is the diet-specific assimilation efficiency of a given trophic group (de Ruiter et al. 1993), $X$ is the summed metabolic rates of all individuals within a trophic group in a given community, and $L$ is the loss of energy from a given biomass pool to higher trophic levels due to predation (Chapter 3 ). We then summed together fluxes among all trophic levels to get a community-level measure of energy flux and compiled dissimilarity matrices from LRR's between total community energy flux values within each region.

\subsubsection{Statistical analyses}

In order to disentangle the roles of different components of biodiversity from pure biomass effects in driving variation in ecosystem functioning across environmental and spatial gradients (figure 5.1), we employed the use of multiple regression on distance matrices (MRM) using the 'ecodist' package in R (R Core Team 2015) within a path modelling framework. Specifically, MRM regresses a response matrix on any number of explanatory matrices composed of dissimilarities or distances, allowing for the inference of how differently measured multi- or univariate variables might influence each other across environmental and spatial distance (Lichstein 2007). Previous studies have proven path modelling to be a highly effective tool for disentangling the complex causal relationships among environmental change, community attributes, and ecosystem 
functioning (Laliberté \& Tylianakis 2012; Barnes et al. 2014b). Here, we use Shipley's (Shipley 2009) d-separation method of generalised causal path analysis, as this method is highly flexible for using a variety of model types, such as MRM's in this case.

To construct the path model, we first identified the basis set $\boldsymbol{B}_{U}$ of independence claims that were implied by our hypothetical causal model (Figure 5.2). To do so, we identified all pairs of variables in the path model with no arrow between them (i.e. relationships that yielded $\mathrm{p}>0.05)$. As such, $\boldsymbol{B}_{U}$ implies the dependence claims (i.e., pairs of variables in the model with a causal arrow joining them) (Shipley 2009). The independence claims in $\boldsymbol{B}_{U}$ describe the $p_{i}$ probability that variable pairs $\left(X_{i}, X_{j}\right)$ are independent conditional on the variable set $Z$, which is a direct cause of either $X_{i}$ or $X_{j}$. The combined $p_{i}$ of the full model was calculated as

$$
C=-2 \sum_{i=1}^{k} \ln \left(p_{i}\right)
$$

and the $C$ value was then compared to a chi-square $\left(\chi^{2}\right)$ distribution with $2 k$ degrees of freedom (Shipley 2009). The resulting probability, $P$, indicates whether the data depart significantly from what would be expected under such a causal model. A model is rejected if the resulting $P$-value is smaller than the specified $\alpha$-level (in this case $\alpha=$ $0.05)$. As such, if $P>0.05$ the causal model is considered to provide a good fit to the data (Shipley 2009).

The path model was constructed to test how spatial and environmental distance can influence the role of four different mechanisms through which biodiversity can drive spatial variation in ecosystem functioning: H1) species composition, H2) $\alpha$-diversity, H3) functional dispersion, and H4) community biomass (Figure 5.1 and Figure 5.2). In addition, we assumed and tested for effects of $\beta$-diversity on $\alpha$-diversity and functional dispersion, as well as effects of these three variables on community biomass (Figure 5.2).

All MRM models were performed with 10,000 permutations to ensure stable estimations of $P$-values that were used to calculate the $C$ statistic in the path models. To assess the relative importance of environmental versus geographical distance for dissimilarity in species composition, $\alpha$-diversity, functional dispersion and biomass, as well as the relative importance of these variables for driving dissimilarity in community energy fluxes, we calculated range-standardized coefficients for each predictor variable as recommended by (Grace 2006). Specifically, this is a standardization of raw coefficients 
$\beta_{x y}$ expressing the effect of $x$ on $y$, whereby the range-standardized coefficient $\beta \operatorname{std}_{x y}=\beta_{x y}$. $\left(\mathrm{x}_{\max }-\mathrm{x}_{\min }\right) /\left(\mathrm{y}_{\max }-\mathrm{y}_{\min }\right)$, where the max and min values are the largest and smallest calculated dissimilarity values from the distance matrices. This method of coefficient standardization yields dimensionless coefficients that can be interpreted as the proportional change in $y$ across the range of $x$ after controlling for all other predictors in the model. All data preparation and statistical analysis was carried out in R 3.1.3 (R Core Team 2015).

\subsection{RESULTS}

In both the Indonesian and German study regions, geographic and environmental distance played an important role in structuring litter macroinvertebrate communities. The strongest spatial and environmental turnover was observed for species composition ( $\beta$ diversity), compared with the other diversity variables across the two regions. Across the sampling sites in Indonesia, we found that environmental distance played a larger role than geographic distance in driving $\beta$-diversity (Figure 5.3a), with species dissimilarity changing by $23 \%$ versus $10 \%$ over its measured range (Supplementary Table 5.2) across the environmental and spatial gradients, respectively. In contrast, geographic distance had a stronger effect on species turnover than environmental distance at the German sites (Figure 5.3b), with a shift in species dissimilarity of 20\% compared to $23 \%$ (Supplementary Table 5.2) across the environmental and spatial gradients, respectively. Despite the combined effects of spatial and environmental distance on $\beta$-diversity, there was relatively low variance in $\beta$-diversity explained by these variables in Indonesia $\left(R^{2}=\right.$ $0.15)$, but considerably higher variance explained at the German sites $\left(R^{2}=0.26\right.$, Figure 5.3). Interestingly, the other measures of macroinvertebrate community structure only responded to geographic distance, with a very weak response of dissimilarity in functional dispersion to geographic distance in Indonesia and similarly weak but significant turnover in $\alpha$-diversity and biomass across the spatial gradient at the German sites (Figure 5.3). 


\section{a) Indonesia}

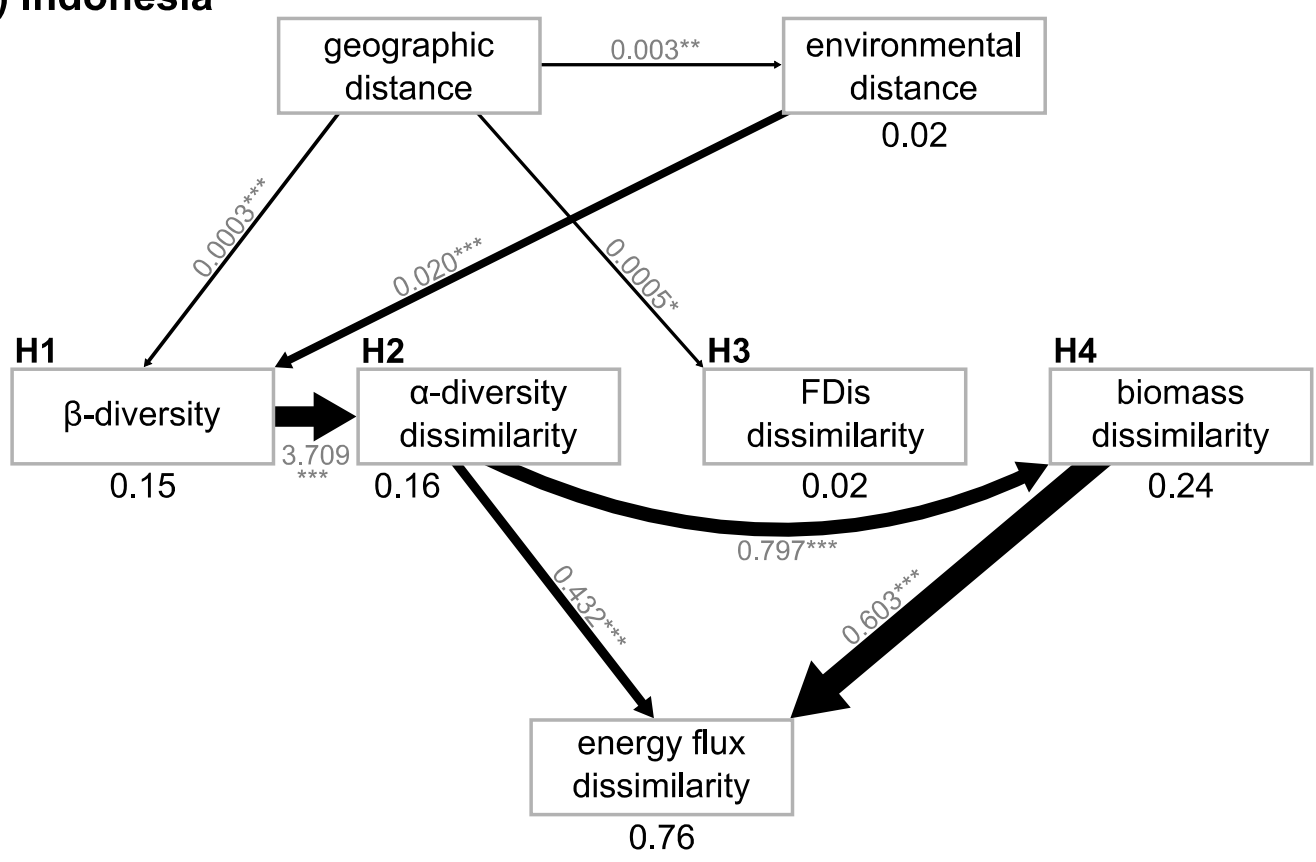

\section{b) Germany}

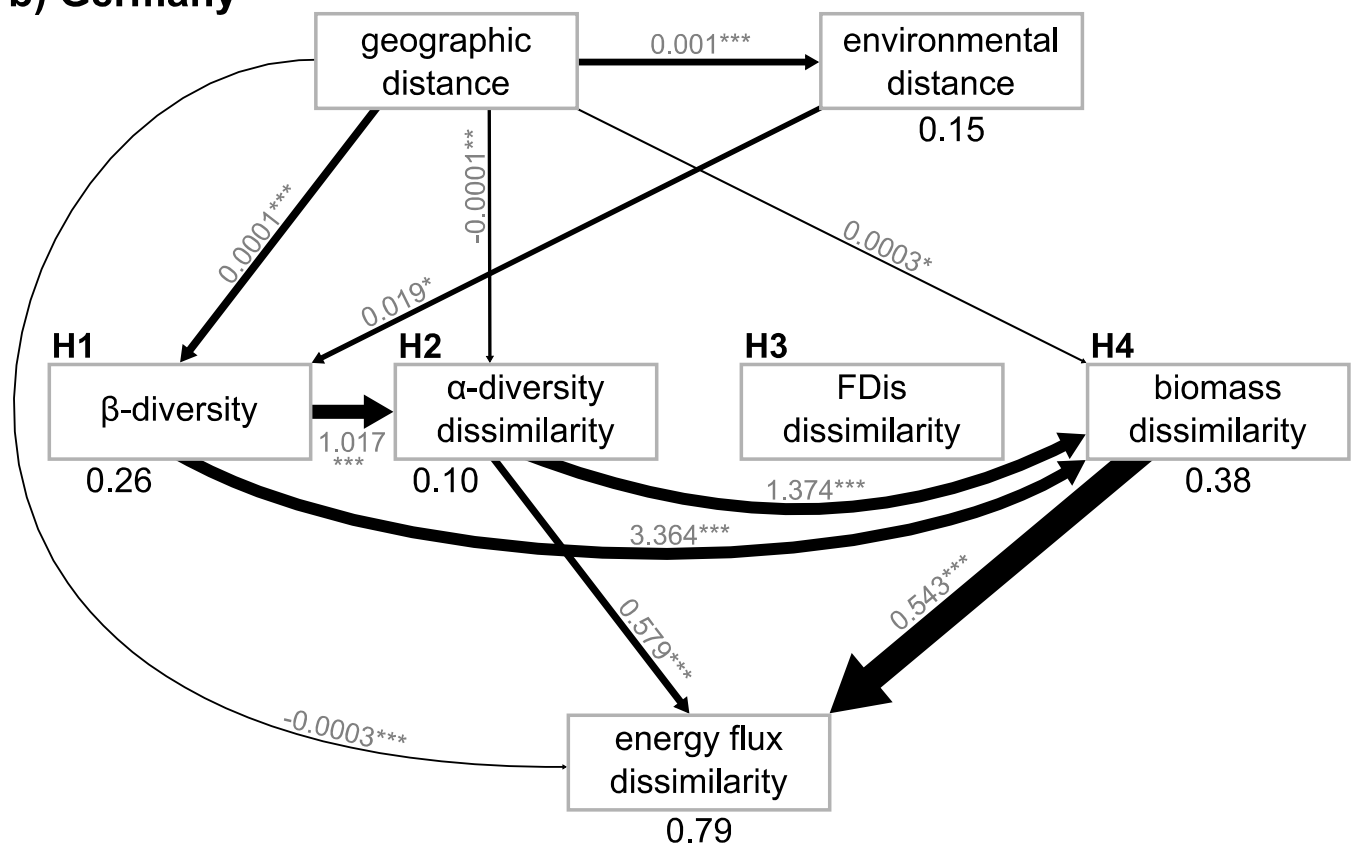

Figure 5.3: Path models constructed from multiple regression on distance matrices (MRM) testing for the different mechanisms that determine spatial variation in energy fluxes of litter macroinvertebrate communities across landscapes in a) Indonesia and b) Germany. H1 - H4 denote hypothesis tests as shown in Fig. 2. 'FDis' stands for functional dispersion. Values under each response variable indicate the $R^{2}$ for each individual MRM. Values next to each arrow are the unstandardised coefficients and arrow widths indicate the range-standardized effect size of each predictor variable. Asterisks denote significance levels: $* \mathrm{p}<0.05 ; * * \mathrm{p}<0.01$; *** $\mathrm{p}<$ 0.001 . 
The high $\beta$-diversity observed across sites in both regions $(71-99 \%$ and $53-$ 98\% species turnover in Indonesia and Germany, respectively), which was partially driven by the spatial and environmental gradients in these sampling regions, was an important determinant of dissimilarity in $\alpha$-diversity and community biomass (Figure 5.3). Specifically, the overall $29 \%$ shift in $\beta$-diversity in Indonesia and $45 \%$ shift in Germany across their measured range directly drove a respective $59 \%$ and $41 \%$ change in $\alpha$-diversity across these study regions. However, the explained variance for turnover in $\alpha$ diversity was low, with an $R^{2}$ of 0.16 in Indonesia and only 0.10 in Germany. We found that community biomass also responded with a $35 \%$ change across the measured range of $\beta$-diversity, but this pattern was only evident at the German sites. Consequently, substantially more variation in biomass turnover could be explained across the German sites $\left(R^{2}=0.38\right)$ compared to the Indonesian sites $\left(R^{2}=0.24\right)$.

Interestingly, the emergence of specific and generalizable mechanisms that drive turnover in ecosystem functioning across sites was clear from our path models. In both regions, we found a clear effect of $\alpha$-diversity and biomass on energy fluxes (Figure 5.3). Specifically, this means that with increasing dissimilarity in the total number of species and total biomass between sampling sites we found a resulting increase in the dissimilarity of energy fluxes of these sites. Across both the Indonesian and German sites, there were relatively similar changes in community energy fluxes across the range of $\alpha$ diversity (27\% in Indonesia and $21 \%$ in Germany). This likeness between the two regions in turnover of energy fluxes also held in response to turnover in biomass, with a 73\% change in energy flux dissimilarity in Indonesia and a 77\% change in Germany across their respective ranges of community biomasses (Figure 5.3; Supplementary Table 5.2). At the German sites only, we found a residual effect of geographic distance on energy flux turnover after controlling for all other predictors in the path model, but this effect was extremely weak (only a $5 \%$ change) compared to those of $\alpha$-diversity and biomass (Figure 5.3; Supplementary Table 5.2). In addition to the clear detection of mechanisms driving spatial turnover in energy flux, we also found that a high proportion of variation in energy flux turnover was explained by these mechanisms (76\% and $79 \%$ in Indonesia and Germany, respectively). 


\subsection{DISCUSSION}

By employing a landscape-level approach across a tropical and temperate region, our study effectively disentangles the mechanisms responsible for driving differences in ecosystem functioning across landscapes. We found that both spatial and environmental distance are important for driving turnover in community composition, leading to clear differences among sampling sites in overall rates of energy flux in multitrophic communities of litter macroinvertebrates. Despite some differences in the relative strength of effects on various community attributes (such as $\alpha$ - and $\beta$-diversity) and energy fluxes, we found remarkable similarities in the mechanisms driving these responses between the Indonesian and German study regions.

As expected, both geographic and environmental distance among plots predicted turnover in species composition, which was clearly evident in both study regions. Interestingly though, environmental distance had a stronger effect on species turnover in the Indonesian communities whereas geographic distance had a stronger effect in the German communities. These findings seemingly contradict those of (Myers et al. 2013), who showed that environmental factors played a stronger role in driving species turnover in a temperate compared with a tropical region. Our results may differ because of the different taxa among the two studies, i.e., plants versus litter macroinvertebrates, as dispersal of these two groups is likely to differ considerably, thus leading to different mechanisms of assembly operating on these organisms. In any case, our findings could provide evidence for greater environmental filtering processes in the tropical Indonesian communities compared to stronger dispersal-dependent random assembly in the temperate German communities (Myers et al. 2013). Alternatively, the larger total geographic extent across the German study region $(630 \mathrm{~km})$ could inherently give rise to overall higher species turnover compared to the Indonesian study region (90 km). Nevertheless, the range of environmental distance among plots was highly similar between the Indonesian study region (0.097 - 3.862 Euclidean distances) and the German study system (0.149 - 3.840 Euclidean distances). Therefore, the 36\% stronger standardized effect of environmental distance on $\beta$-diversity in Indonesia compared with Germany quite likely indicates that the tropical communities are more subject to environmental filtering than the temperate communities.

We found that turnover in species composition ( $\beta$-diversity) strongly predicted dissimilarity in total species richness among communities ( $\alpha$-diversity) in both of the 
study regions. This result could suggest that coexistence of species at the local scale might depend on functional differences among species that allow them to utilise resources differently (Kneitel \& Chase 2004). In other words, particular species assemblages are likely to give rise to greater numbers of species in assemblages where antagonistic interactions, such as predation and competition, are weaker. As such, we would expect $\beta$ diversity to predict dissimilarity of $\alpha$-diversity among plots. However, these results should be interpreted with caution, since $\alpha$-diversity and $\beta$-diversity are typically highly correlated when turnover is calculated using Jaccard dissimilarities (Chao, Chazdon \& Shen 2005). In both the Indonesian and German study regions, increasing dissimilarity among communities also resulted in greater among-plot dissimilarity in total biomass. These results are confirmative of a multitude of previous studies showing that species richness drives patterns in productivity, especially for primary producer organisms (e.g., Tilman et al. 2001; Cardinale et al. 2013). Interestingly, we also found an effect of species composition on community biomass in the German litter communities suggesting that the combined identities of particular species might be driving varying levels of biomass across these landscapes. For example, the occurrences of particular species that are competitively dominant where particular resources are available are likely to drive locally increased biomass in European forests (Salamon et al. 2006).

Perhaps most strikingly, we found highly consistent patterns between the tropical and temperate study regions in the mechanisms that were directly responsible for spatial variation in ecosystem functioning. In both study regions, we found that dissimilarity in biomass and species richness among plots was highly important for dissimilarity in energy flux. In fact, even the standardized effect size of these variables on energy flux were almost identical between the two regions, with changes in energy flux turnover of $73 \%$ in Indonesia versus $77 \%$ in Germany in response to biomass turnover, and $27 \%$ versus $21 \%$ changes in energy flux turnover (in Indonesia and Germany, respectively) in response to $\alpha$-diversity turnover. These results strongly support the species richness $(\mathrm{H} 2)$ and biomass (H4) hypotheses. Regarding the species richness hypothesis, it is likely that increasing species richness of potential prey in litter invertebrate communities allows for increased resource exploitation by higher trophic-level consumers (Gamfeldt, Hillebrand \& Jonsson 2005). As Gamfeldt et al. (2005) demonstrate experimentally, this should also result in higher biomasses of consumer species and, thus, overall higher community biomass. As such, dissimilarity in $\alpha$-diversity among communities should also drive dissimilarity in biomass; a pattern which our path models both strongly support. 
Furthermore, after holding constant any effects of species richness on energy flux, spatial variation in litter invertebrate biomass still had a very strong effect on spatial variation in energy flux indicating that, regardless of any resource diversity effects, total biomass of organisms expectedly plays an important role in determining ecosystem process rates (Barnes et al. 2014b).

Although we did not find any direct effects of functional dispersion (H3) or $\beta$ diversity $(\mathrm{H} 1)$ on spatial variation in energy fluxes, this does not necessarily mean that these factors do not play a role in shaping spatial patterns in ecosystem functioning in litter macroinvertebrate communities. On the contrary, our path models indicate that there were indirect effects of species turnover on energy flux via spatial variation in $\alpha$ diversity, likely resulting from altered patterns in coexistence of various species in both the tropical and temperate communities (Kneitel \& Chase 2004). Therefore, although these indirect effects of $\beta$-diversity do not lend support to the species composition hypothesis (H1), our results do indicate that there are multiple interacting mechanisms that drive spatial variability of ecosystem functioning in real-world systems.

Nevertheless, our results also indicate that a very simplistic set of predictors, i.e., species richness and total biomass, may provide the strongest predictive power for ecosystem functioning at the landscape scale.

\subsubsection{Conclusions}

Our study provides new insight into the mechanisms that determine spatial patterns in multitrophic biodiversity and ecosystem functioning by confirming results across both tropical and temperate landscapes. Despite some minor differences among our two study regions in the mechanisms driving spatial variation in ecosystem functioning, we find remarkable similarity from the tropical to temperate systems, indicating that globally consistent and generalizable patterns in biodiversity and ecosystem functioning relationships at the landscape scale likely exist. These results call for various new avenues of BEF research, such as the extension of landscape-level mechanistic tests of BEF relationships to freshwater, marine, and other terrestrial ecosystems, as the spatial dynamics of these systems could vary (Soininen, Lennon \& Hillebrand 2007). Moreover, investigating spatial variation in energy flux among trophic levels could shed light on how trait-dependent loss of biodiversity could lead to rapid decay of ecosystem services at larger spatial scales (Larsen et al. 2005). In recent years, the merging of food web ecology and BEF research has moved toward centre stage (Reiss et al. 2009) due to the 
enhancement of predictive accuracy with increased ecological complexity. Bringing these merged fields of ecology into the arena of spatial ecology presents an exciting new frontier in the exploration of biodiversity-ecosystem functioning relationships.

\subsection{ACKNOWLEDGEMENTS}

We thank Megawati, Rizky Nazarreta, Keisha Disa Putirama, Rosario Reza Valentino Lasse for assistance in the field and laboratory, Ana Meijide for providing climate data, Kara Allen for providing soil $\mathrm{pH}$ data, and Martyna Kowtowska for providing litter depth data for the Indonesian sites. We would also like to thank Helmut Hillebrand for helpful comments on earlier versions of this manuscript. Also special thanks to Roswitha Ehnes for providing additional metabolic rate regression parameters, as well as to the Indonesian village leaders, local site owners, PT REKI, and Bukit Duabelas National Park for granting us access to their properties. This study was financed by the Deutsche Forschungsgemeinschaft (DFG) in the framework of the collaborative German Indonesian research project CRC990 and the Ministry of Science and Culture of Lower Saxony within the framework of the BEFmate project. 


\section{Part III}

General Discussion 


\section{Chapter 6}

\section{Synthesis}

\subsection{SYNOPSIS}

With the increasing expansion and intensification of anthropogenic land use, natural ecosystems face ever greater peril (Foley et al. 2005). A continuously rising human population is one of the main drivers of this environmental pressure, due to concurrent demands for natural resources (Ehrlich \& Ehrlich 2013). This growing demand has resulted in the intensification of agriculture in developed nations and especially severe agricultural expansion coupled with deforestation in developing tropical countries (Matson et al. 1997; Lambin \& Meyfroidt 2011; Laurance et al. 2014). We are now beginning to see more contemporary trends in the intensification of tropical land-use systems, such as through the conversion of agroforests to large-scale monoculture rubber and oil palm plantations (Wilcove et al. 2013). While the mechanisms that determine trajectories of ecological change following land-use conversion and intensification are virtually innumerable, there is an increasingly fundamental need to quantify the role of these mechanisms. Only by understanding these mechanistic processes can we hope to develop a framework that allows for the prediction of realistic future global-change scenarios (Pereira et al. 2010).

There has generally been a focused concern over the loss of biodiversity in response to global environmental change, particularly regarding the impacts of land-use change (Sala et al. 2000; Cardinale et al. 2006; Gardner et al. 2009; Pimm et al. 2014). It is becoming increasingly evident that not only losses in biodiversity but also alterations of population biomass and community composition are collectively driving important shifts in the way that species interact in complex ecosystems (Tylianakis et al. 2008a). Anthropogenically driven modification of species interactions can alter the response of ecological communities to disturbances by indirectly altering multiple interacting species, such as through trophic cascades (Pace et al. 1999; Frank et al. 2005; Jochum et al. 2012). Species interactions are also fundamental for the provisioning of ecosystem 
functions as they regulate the transfer of energy across trophic levels (Chapin III et al. 2000; Duffy et al. 2007; Reiss et al. 2009). Therefore, accounting for species interactions in anthropogenically altered ecosystems is crucial if we are to grasp the scale of land-use impacts across multiple taxa. In addition, variation among species in their functional traits gives rise to variability in the way that species interact (Thompson et al. 2012), how they respond to environmental change (McGill et al. 2006), the influence that these species have on ecosystem processes (Larsen et al. 2005), and the resilience and stability of whole species assemblages to future ecological perturbations (Mori et al. 2013). Together, accounting for species interactions as well as functional traits can therefore be a powerful approach for investigating land-use impacts on ecological communities and how these impacts will alter the provisioning and stability of ecosystem functions. Species richness has been repeatedly shown to be an important driving mechanism in the provisioning and stability of ecosystem functioning (Balvanera et al. 2006). Despite this, the role of different mechanisms for driving ecosystem functioning at the landscape scale is poorly understood, even though land-use impacts on biodiversity occur from the local to global scale (Foley et al. 2005; Newbold et al. 2015).

In this thesis I investigated the impacts of tropical land-use intensification on complex multitrophic communities by firstly determining whether bottom-up trophic interactions mediate land-use effects, or if these effects are rather directly imposed at different trophic levels across a broad range of taxa in Sumatra, Indonesia (Chapter 2). I demonstrated that the importance of trophic interactions increased with trophic level, whereby impacts of land-use intensification shifted from being mostly direct at lower trophic levels to predominantly indirect at the highest trophic levels. After establishing the importance of trophic interactions for mediating the response of these tropical ecosystems to land-use intensification, I tested how the alteration of litter invertebrate communities leads to losses in ecosystem functioning across multiple trophic levels (Chapter 3). Specifically, by combining aspects of metabolic theory (Ehnes et al. 2011) with food web theory (de Ruiter et al. 1994), I analytically calculated whole-community energy flux and demonstrated that, through the alteration of community structure and species diversity, land-use intensification to oil palm agriculture brought about large reductions in multitrophic ecosystem functioning. Following this discovery, I employed a trait-based approach to measure the response diversity and functional redundancy of these litter macroinvertebrate communities (Chapter 4). I found that in more intensive land-use systems, communities appeared to be more randomly assembled in conjunction with 
having reduced species richness. This resulted in an overall reduction in functional redundancy, indicating that invertebrate communities in intensified land-use systems such as oil palm plantations may be at risk of drastic losses in ecosystem functioning. Finally, I employed the methods developed in Chapter 3 for calculating multitrophic energy flux of litter invertebrate communities in tropical and temperate ecosystems to disentangle the mechanisms that drive variation in ecosystem functioning across spatial and environmental gradients. In doing so, I discovered that more complex measures of biodiversity (i.e., functional trait dispersion and species composition) that are related to niche complementarity and identity effects played no important role in explaining variability in ecosystem functioning. Instead, the simplest measures of community composition i.e., species richness and community biomass were clearly the most important drivers of variation in ecosystem functioning.

\subsection{DISCUSSION}

While there has been considerable interest in the impacts of global change on species interactions through the occurrence of trophic cascades (Pace et al. 1999), there has been a lack of studies that mechanistically test for such processes across a large range of trophic levels (Tylianakis et al. 2008a). Furthermore, a clear understanding of how trophic cascades play a role in the response of multitrophic communities to tropical landuse change is virtually absent (Gardner et al. 2009; Tscharntke et al. 2012b). In Chapter 2, based on previously established evidence for extensive bottom-up effects following manipulations of primary producers (Scherber et al. 2010), I developed two main hypotheses to explain how multitrophic communities will respond to land-use intensification: 1) the direct land-use effects hypothesis and 2) the cascading land-use effects hypothesis. Unlike previous studies that tested for the occurrence of trophic cascades in response to environmental perturbations, I employed a structural equation modelling (SEM) framework (Grace 2006) to test for causal trophic pathways of community responses throughout a broad range of trophic levels across variably intensified tropical land-use systems. An immediately evident pattern from this SEM was that land-use intensification imposed very strong, direct impacts on almost all trophic levels except for predators, with especially strong effects on below-ground taxa. This is quite likely because of the expected strong effects of intensive management practices 
such as fertilisation and pesticide application on soil microbes and invertebrates (Mäder et al. 2002; Zhang et al. 2009; Geiger et al. 2010) as well as on plant communities (Hautier et al. 2009). Within the SEM framework, I allowed for the interaction between background environmental variation and land-use effects, which demonstrated that the impacts of land-use intensification are somewhat dependent on the environmental context of a given location, such that soil properties either diminished or exacerbated land-use effects on plant and litter invertebrate communities. Interestingly, I found that birds did not exhibit strong responses to either direct or indirect land-use effects, with the exception of predatory birds that were affected by their prey's diversity and abundance. This result points to the issue of using highly mobile taxa such as birds for local-level investigations of land-use intensification, as these taxa are more likely to be affected by larger landscape-level processes (Kremen et al. 2007; Gabriel et al. 2010). Of particular interest was the shift toward the importance of bottom-up trophic effects with increasing trophic level in this system. This finding raises the question of why the highest trophic level groups were hardly affected by land-use intensification but strongly controlled by their resources, compared with the lower trophic levels. Quite likely, this occurs because higher trophic levels are dependent on more trophic levels beneath them, so even if certain predatory species might be resilient to direct land-use impacts, they will still be at risk of extinction if their resources are strongly affected (Purvis et al. 2000). Results from this study suggest that the detection of trophic cascades in ecosystems following land-use intensification will, at least in part, depend on the trophic breadth of the taxa sampled. This is because both direct and cascading land-use effects structure respondent communities, but the importance of these effects scale with trophic level. In conclusion, conservation efforts at lower trophic levels will likely benefit from focusing on direct land-use impacts such as agrochemical inputs, whereas conservation of higher trophic level taxa will depend on the maintenance of their resources, pointing to the overall efficacy of a bottom-up management strategy.

In Chapter 3, my aim was to take into account the clearly established importance of trophic interactions in ecosystems to determine how tropical land-use change impacts ecosystem functioning of multitrophic communities. In order to answer this question, I narrowed the taxonomic scope to litter macroinvertebrate communities and developed a measure of ecosystem functioning that could be calculated for multitrophic communities, irrespective of the number of trophic levels. By combining concepts from the metabolic theory of ecology (Brown et al. 2004) and food web theory (de Ruiter et al. 1993, 1994), 
I calculated the flux of energy from each resource biomass pool to the respective consumer biomass pools. In doing so, this measure essentially captured a dynamic ecological process that is dependent on the combined metabolic demands of the consumer populations, the assimilation efficiencies of these populations, and also the loss of energy to higher trophic levels. As such, this measure accounts for the energetic demands of organisms, their ability to digestively assimilate energy from their food, as well as the trophic topology of the community that these organisms are a part of. By summing up the fluxes of energy to all biomass pools in each community, I was consequently able to test for the effects of land-use type on multitrophic biodiversity and ecosystem functioning without any restriction to a particular trophic level. I found that different trophic groups responded differently to land-use change, whereby predators and detritivores decreased strongly in biomass from forest to oil palm plantations, compared with a slight increase in omnivore biomass. In contrast, however, energy fluxes for all trophic groups declined strongly from the forest to oil palm systems. These results indicate that biomass may not necessarily provide an accurate surrogate for the processing of resources and energy uptake of consumers, despite the fact that biomass has been a very widely applied measure of function in biodiversity-ecosystem functioning (BEF) research (Hooper et al. 2005; Balvanera et al. 2006). Interestingly, by regressing community energy flux on species richness at the whole community level, I found a significantly steeper increase in energy flux within the oil palm plantations compared to the other less intensive land-use systems, suggesting low functional redundancy in oil palm plantations (Naeem \& Wright 2003). In addition, I compared this overall relationship against BEF relationships of individual trophic groups, which clearly demonstrated that BEF relationships are dependent on the trophic group in question. Therefore, this chapter provides strong support for the importance of incorporating trophic complexity into BEF research (Duffy et al. 2007).

The striking results that emerged from developing a fully multitrophic measure of ecosystem functioning stimulated the investigation of how biodiversity loss in litter communities might compromise functional stability within intensified agriculture, as well as the mechanisms underlying BEF relationships in multitrophic communities at large spatial scales (Chapter 4 and 5). Theory suggests that at high levels of species richness, the provisioning of ecosystem functions is likely to be more stable because there are more functionally similar species that can maintain an ecosystem function should other species be lost from the ecosystem (Naeem \& Wright 2003). In addition, communities composed 
of more functionally dissimilar species should theoretically be more resilient to a variety of ecological disturbances (Mori et al. 2013). To quantify the resilience and functional stability of litter macroinvertebrate communities in intensified tropical land-use systems, I used species traits to test for the likely mechanisms driving community assembly in different systems (Mouchet et al. 2010). I found that oil palm communities appeared to be assembled more randomly than in the other less intensively managed systems. This was evident by an overall higher community-level dispersion of traits, which is indicative of weak ecological filtering acting on these species assemblages. Furthermore, I calculated response diversity by measuring the average trait dispersion within functional response groups (based on traits that determine a species' response to disturbances) and functional redundancy by calculating the mean number of species within functional effect groups (based on traits that determine a species' influence on ecosystem processes). These measures gave an indication of community resilience and functional stability at each sampling site. Similar to a previous study on plant communities (Laliberté et al. 2010), I found that litter invertebrate communities in the more intensively managed oil palm plantations had considerably lower functional redundancy than in the less intensive systems. This finding shows that in multitrophic invertebrate communities, the propensity for losses in entire ecosystem functions rises with increasing land-use intensification, given future ecological perturbations. Moreover, I found that intermediate-intensity agricultural systems such as jungle rubber agroforestry as in this study are likely to act as reservoirs of functional stability in human-dominated landscapes. These results highlight the importance of interspersing these low-intensity agroforestry systems in expansive monoculture-dominated landscapes in order to maintain the provisioning of crucial ecosystem services such as nutrient cycling and biological control.

One of the most important challenges in contemporary BEF research has been to relate findings from small-scale experimental studies to larger-scale real-world scenarios in determining the underlying mechanisms that drive variation in ecosystem functioning (Duffy 2009; Cardinale et al. 2012). This is, perhaps, partly due to the constraints of measuring ecosystem functioning - especially in complex multitrophic communities - at larger scales in uncontrolled experiments. Essentially, by developing an analytical measure of multitrophic ecosystem functioning, I was able to overcome this limitation. This allowed me to test for the mechanistic drivers of spatial variation in ecosystem functioning across multiple landscapes in Indonesia and compare these results to similarly sampled litter invertebrate communities across multiple landscapes in Germany. By once 
more employing an SEM framework (Grace 2006), but this time based on distance matrices (Lichstein 2007), I developed a series of hypotheses that could be simultaneously tested in order to discern the mechanisms that give rise to spatial variability in ecosystem functioning. In doing so, these analyses revealed that environmental distance seemed to play a greater role in determining species turnover in the Indonesian landscapes than in Germany, with little difference in the effect of geographic distance between regions. This was surprisingly in contrast to a previous study on plant communities that found the converse pattern between a tropical and temperate region (Myers et al. 2013), suggesting that different assembly mechanisms might operate on different taxonomic groups depending on their mobility. Nevertheless, species turnover seemed to play no part in driving spatial variability in community energy flux, thus providing no support for the notion that species' identities influence the provisioning of ecosystem functioning at the landscape scale. Similarly, functional dispersion had no apparent effect on the turnover of energy flux, also pointing to the triviality of niche complementarity at larger spatial scales. In contrast, I found that the two most simple measures of community structure i.e., species richness and community biomass, were responsible for explaining spatial variation in energy flux. Taken together, these results suggest that if one is to scale up measured ecosystem functions from the local- to landscape-scale, the key co-variables to be considered for litter macroinvertebrate communities would be species richness and biomass. Through the novel advance in developing an easily calculable measure of ecosystem functioning for multitrophic communities, combined with the integration of distance matrices within a flexible SEM framework, this chapter provides new insight into the underlying mechanisms of BEF relationships at large spatial scales.

\subsection{FUTURE PROSPECTS AND CHALLENGES}

By drawing from broad areas of ecological theory such as the ecology of global change, food web ecology, metabolic theory, and biodiversity-ecosystem functioning theory, this thesis presents a significant advance in understanding of how complex ecosystems will respond to anthropogenic land use. The application of cutting-edge statistical techniques such as the flexible multi-level path modelling (SEM) in Chapters 2 and 5, as well as the development of a highly versatile measure of multitrophic ecosystem functioning were 
instrumental in bringing about the advances presented here. The results from the novel investigations in this thesis inherently give rise to a whole new suite of unanswered questions that present many exciting new directions for future research.

In Chapter 2, the finding that plants appear to drive negative effects within the less intensive land-use systems might imply that the bottom-up effects propagating upward from primary producers could be dependent on the total species richness of the system. In order to test this notion, experimental field plots could be established in high-diversity systems such as tropical rainforests and agroforest systems where plant diversity and biomass could then be manipulated. This would complement the similar studies already carried out in relatively low-diversity grassland systems (Haddad et al. 2009; Scherber et al. 2010; Borer et al. 2012), providing the capacity to assess whether positive bottom-up effects typically found in temperate grassland studies can be generalised to high-diversity ecosystems. Furthermore, the generalisability of the findings from these studies could be greatly increased by accounting for other potential interacting global change drivers. For example, habitat fragmentation and climate change are expected to synergistically interact by reducing the ability of organisms to move through fragmented landscapes in order to track rising temperatures (Hof et al. 2011). This interaction has been shown behaviourally in a microcosm experiment (Barnes et al. 2015) in the absence of species interactions. However, field experiments that combine warming treatments (e.g., Brzostek et al. 2012; Eisenhauer et al. 2012) with experimentally fragmented plots, along with varying levels of land-use intensification (by altering levels of pesticide and fertiliser applications) could then utilise a similar analytical approach as in Chapter 2. This would greatly advance our understanding of how multiple interacting drivers of ecological change impact ecosystems that are structured by species interactions.

In addition to measuring species diversity and biomass in communities subjected to experimental warming and land-use intensification, the investigation of ecosystem functioning responses will provide important insights into the future provisioning of ecosystem services in intensifying agricultural landscapes. In particular, increasing temperatures due to climate change will likely influence energy flux (Chapter 3 and 5) among trophic levels. This is because metabolic rates (Brown et al. 2004) and assimilation efficiencies (Lang et al. unpublished data) scale positively with temperature. Therefore, these physiological temperature dependencies could result in unforeseen consequences for energy flux. This presents the intriguing possibility of investigating combined effects of land-use intensification and warming in experimental plots, as 
discussed above, on rates of community energy flux across multitrophic communities. Perhaps one of the main challenges for increasing the accuracy of this approach to measuring energy flux is the refinement in trophic structure to better estimate realistic losses of energy to predation. However, with the increasing focus of studies on global change drivers toward species interactions, datasets that have fairly resolved trophic topologies are becoming more common (Tylianakis et al. 2008a). Therefore, the ability to incorporate more detailed trophic structures into energy flux calculations is within reach.

In Chapter 4, I demonstrated a greater likelihood of functional collapse in litter invertebrate communities inhabiting intensively managed agricultural systems, as has been similarly shown in plant communities (Laliberté et al. 2010). These findings introduce very important unanswered questions: i.e., what kind of future perturbations will yield functional collapses, how strong do these perturbations need to be, and what scale of functional collapse are these communities facing as a result? A modelling approach would provide a strong framework under which these questions could be explored. For example, by altering the type of environmental perturbation, such as an extreme climatic event or the introduction of an invasive species, one could test for thresholds of losses in functional groups resulting from species extinctions. Under such a framework, the level of functional dispersion and number of species within functional groups (i.e., response diversity and functional redundancy, respectively) could be varied along with changes in the intensity and type of perturbation. In addition, using the method developed in Chapter 3 to calculate multitrophic energy fluxes, actual measures of wholecommunity ecosystem functioning could be calculated for model communities that would indicate the expected provisioning of ecosystem functioning for communities following losses in functional diversity. Such modelling experiments would provide important insight into exactly how prone ecosystems are to functional collapses under different global change scenarios by elucidating the boundaries of "safe" operating spaces for communities that have previously undergone varying levels of species loss.

By expanding the development of a widely applicable measure of multitrophic energy flux to the landscape scale, I was able to determine the most important mechanisms of community composition that drive variability in ecosystem functioning at larger spatial scales. In particular, the comparison of the litter macroinvertebrate dataset from Indonesia with a similar dataset from Germany illuminated intriguing possibilities for future exploration; namely, whether BEF relationships change across geographical biomes or, more generally, across a latitudinal gradient. Perhaps one of the greatest 
unanswered questions in ecology is why there is decreasing species richness with increasing latitude (Pianka 1966; Brown 2014). Strictly speaking, according to BEF theory (Naeem \& Wright 2003), this latitudinal gradient in species diversity should result in a similar latitudinal gradient in ecosystem functioning. However, there are many factors that differ which could mediate the prevalence of such a gradient. For example, higher temperatures in the tropics could drive higher rates in energy flux due to increased metabolic rates. The notion of such a latitudinal gradient in ecosystem functioning is virtually unexplored, but with the application of analytically calculated energy fluxes, such global patterns can be mechanistically investigated. Additionally, species-area relationships (SARs) provide a strong framework for understanding how many species can persist in differently sized habitats, thus providing a strong tool for understanding global-change impacts on ecosystems (Thomas et al. 2004; Rybicki \& Hanski 2013). Taking into account the conceptual advances from Chapters 3 and 5 in measuring multitrophic ecosystem functioning and the potential application across different spatial scales, it is possible that the same principles of SARs could be applied to energy fluxes. In principle, this could allow for the estimation of ecosystem functioning of landscapes or even geographic regions, as long as the area-functioning relationship is correctly parameterised by covarying species- and biomass-area relationships. This potential avenue of research could open the possibility of estimating landscape-level ecosystem functioning under varying land-use regimes whilst taking into account factors such as landscape heterogeneity or habitat fragmentation that operate at large spatial scales (Fahrig et al. 2011; Tscharntke et al. 2012b).

With current rates of biodiversity loss and the parallel degradation of ecosystem services, studies that elucidate generalised patterns in ecosystem responses to global environmental change are evermore crucial for developing strategies to mitigate these losses. By integrating across a broad range of ecological concepts and incorporating realworld ecological complexity, the research chapters and synthesis of this thesis present an important contribution to this elucidation. While considerable gaps in scientific understanding have been filled by this contribution, it has also brought to light other important areas that require further exploration. In doing so, this thesis illuminates exciting challenges and opportunities that lie on the horizon for research investigating the impacts of global change drivers on the structure and functioning of complex ecosystems. 


\section{Part IV}

Appendix 


\section{BIBLIOGRAPHY}

Abood, S.A., Lee, J.S.H., Burivalova, Z., Garcia-Ulloa, J. \& Koh, L.P. (2014) Relative contributions of the logging, fiber, oil palm, and mining industries to forest loss in Indonesia. Conservation Letters, 8, 58-67.

Allen, K., Corre, M.D., Tjoa, A. \& Veldkamp, E. (2015) Soil nitrogen-cycling responses to conversion of lowland forests to oil palm and rubber plantations in Sumatra, Indonesia. PLOS ONE, 10, e0133325.

Anderson, M.J., Crist, T.O., Chase, J.M., Vellend, M., Inouye, B.D., Freestone, A.L., Sanders, N.J., Cornell, H. V, Comita, L.S., Davies, K.F., Harrison, S.P., Kraft, N.J.B., Stegen, J.C. \& Swenson, N.G. (2011) Navigating the multiple meanings of $\beta$ diversity: a roadmap for the practicing ecologist. Ecology Letters, 14, 19-28.

Asari, N., Suratman, M.N., Jaafar, J. \& Khalid, M.M. (2013) Estimation of above ground biomass for oil palm plantations using allometric equations. International Proceedings of Chemical, Biological and Environmental Engineering (IPCBEE), 58, 110-114.

Audino, L.D., Louzada, J. \& Comita, L. (2014) Dung beetles as indicators of tropical forest restoration success: is it possible to recover species and functional diversity? Biological Conservation, 169, 248-257.

Balvanera, P., Pfisterer, A.B., Buchmann, N., He, J.-S., Nakashizuka, T., Raffaelli, D. \& Schmid, B. (2006) Quantifying the evidence for biodiversity effects on ecosystem functioning and services. Ecology letters, 9, 1146-56.

Barnes, A.D., Emberson, R.M., Chapman, H.M., Krell, F.-T. \& Didham, R.K. (2014a) Matrix habitat restoration alters dung beetle species responses across tropical forest edges. Biological Conservation, 170, 28-37.

Barnes, A.D., Emberson, R.M., Krell, F.-T. \& Didham, R.K. (2014b) The role of species traits in mediating functional recovery during matrix restoration. PloS ONE, 9, e115385.

Barnes, A.D., Spey, I., Rohde, L., Brose, U. \& Dell, A.I. (2015) Individual behaviour mediates effects of warming on movement across a fragmented landscape. Functional Ecology, in press.

Barnosky, A.D., Matzke, N., Tomiya, S., Wogan, G.O.U., Swartz, B., Quental, T.B., Marshall, C., McGuire, J.L., Lindsey, E.L., Maguire, K.C., Mersey, B. \& Ferrer, E.A. (2011) Has the Earth's sixth mass extinction already arrived? Nature, 471, 5157.

Barragán, F., Moreno, C.E., Escobar, F., Halffter, G. \& Navarrete, D. (2011) Negative impacts of human land use on dung beetle functional diversity. PLOS ONE, 6. 
Basset, Y., Cizek, L., Cuenoud, P., Didham, R.K., Guilhaumon, F., Missa, O., Novotny, V., Odegaard, F., Roslin, T., Schmidl, J., Tishechkin, A.K., Winchester, N.N., Roubik, D.W., Aberlenc, H.P., Bail, J., Barrios, H., Bridle, J.R., Castano-Meneses, G., Corbara, B., Curletti, G., da Rocha, W.D., de Bakker, D., Delabie, J.H.C., Dejean, A., Fagan, L.L., Floren, A., Kitching, R.L., Medianero, E., Miller, S.E., de Oliveira, E.G., Orivel, J., Pollet, M., Rapp, M., Ribeiro, S.P., Roisin, Y., Schmidt, J.B., Sorensen, L. \& Leponce, M. (2012) Arthropod Diversity in a Tropical Forest. Science, 338, 1481-1484.

Batáry, P., Báldi, A., Kleijn, D. \& Tscharntke, T. (2011) Landscape-moderated biodiversity effects of agri-environmental management: a meta-analysis. Proceedings of the Royal Society B: Biological Sciences, 278, 1894-1902.

Bengtsson, J. (1998) Which species? What kind of diversity? Which ecosystem function? Some problems in studies of relations between biodiversity and ecosystem function. Applied Soil Ecology, 10, 191-199.

Bestelmeyer, B.T. \& Wiens, J.A. (1996) The effects of land use on the structure of ground-foraging ant communities in the Argentine Chaco. Ecological Applications, 6, 1225-1240.

Billeter, R., Liira, J., Bailey, D., Bugter, R., Arens, P., Augenstein, I., Aviron, S., Baudry, J., Bukacek, R., Burel, F., Cerny, M., De Blust, G., De Cock, R., Diekötter, T., Dietz, H., Dirksen, J., Dormann, C., Durka, W., Frenzel, M., Hamersky, R., Hendrickx, F., Herzog, F., Klotz, S., Koolstra, B., Lausch, A., Le Coeur, D., Maelfait, J.P., Opdam, P., Roubalova, M., Schermann, A., Schermann, N., Schmidt, T., Schweiger, O., Smulders, M.J.M., Speelmans, M., Simova, P., Verboom, J., Van Wingerden, W.K.R.E., Zobel, M. \& Edwards, P.J. (2008) Indicators for biodiversity in agricultural landscapes: A pan-European study. Journal of Applied Ecology, 45, $141-150$.

Blake, G. \& Hartge, K. (1986) Bulk density. Methods of soil analysis, Part 1 (ed A. Klute), pp. 363-375. Soil Science Society of America, Madison, WI.

Borer, E.T., Seabloom, E.W., Tilman, D. \& Novotny, V. (2012) Plant diversity controls arthropod biomass and temporal stability. Ecology letters, 15, 1457-64.

Bragg, L., Stone, G., Imelfort, M., Hugenholtz, P. \& Tyson, G.W. (2012) Fast, accurate error-correction of amplicon pyrosequences using Acacia. Nature Methods, 9, 425426.

Bray, R.H. \& Kurtz, L.T. (1945) Determination of total, organic, and available forms of phosphorus in soils. Soil Science, 59.

Brookes, P.C., Landman, A., Pruden, G. \& Jenkinson, D.S. (1985) Chloroform fumigation and the release of soil nitrogen: a rapid direct extraction method to measure microbial biomass nitrogen in soil. Soil Biology \& Biochemistry, 17, 837842. 
Brose, U., Martinez, N.D. \& Williams, R.J. (2003) Estimating species richness: sensitivity to sample coverage and insensitivity to spatial patterns. Ecology, 84, 2364-2377.

Brown, J.H. (2014) Why are there so many species in the tropics? Journal of Biogeography, 41, 8-22.

Brown, J.H., Gillooly, J.F., Allen, A.P., Savage, V.M. \& West, G.B. (2004) Toward a metabolic theory of ecology. Ecology, 85, 1771-1789.

Brzostek, E.R., Blair, J.M., Dukes, J.S., Frey, S.D., Hobbie, S.E., Melillo, J.M., Mitchell, R.J., Pendall, E., Reich, P.B., Shaver, G.R., Stefanski, A., Tjoelker, M.G. \& Finzi, A.C. (2012) The effect of experimental warming and precipitation change on proteolytic enzyme activity: positive feedbacks to nitrogen availability are not universal. Global Change Biology, 18, 2617-2625.

Byrnes, J.E.K., Gamfeldt, L., Isbell, F., Lefcheck, J.S., Griffin, J.N., Hector, A., Cardinale, B.J., Hooper, D.U., Dee, L.E. \& Emmett Duffy, J. (2014) Investigating the relationship between biodiversity and ecosystem multifunctionality: challenges and solutions. Methods in Ecology and Evolution, 5, 111-124.

Cadotte, M.W., Carscadden, K. \& Mirotchnick, N. (2011) Beyond species: Functional diversity and the maintenance of ecological processes and services. Journal of Applied Ecology, 48, 1079-1087.

Cagnolo, L., Valladares, G., Salvo, A., Cabido, M. \& Zak, M. (2009) Habitat fragmentation and species loss across three interacting trophic levels: effects of lifehistory and food-web traits. Conservation Biology, 23, 1167-1175.

Campbell, R.E., Harding, J.S., Ewers, R.M., Thorpe, S. \& Didham, R.K. (2011) Production land use alters edge response functions in remnant forest invertebrate communities. Ecological Applications, 21, 3147-3161.

Caporaso, J.G., Kuczynski, J., Stombaugh, J., Bittinger, K., Bushman, F.D., Costello, E.K., Fierer, N., Peña, A.G., Goodrich, J.K., Gordon, J.I., Huttley, G.A., Kelley, S.T., Knights, D., Koenig, J.E., Ley, R.E., Lozupone, C.A., McDonald, D., Muegge, B.D., Pirrung, M., Reeder, J., Sevinsky, J.R., Turnbaugh, P.J., Walters, W.A., Widmann, J., Yatsunenko, T., Zaneveld, J. \& Knight, R. (2010) QIIME allows analysis of high-throughput community sequencing data. Nature methods, 7, 335336.

Cardinale, B.J., Duffy, J.E., Gonzalez, A., Hooper, D.U., Perrings, C., Venail, P., Narwani, A., Mace, G.M., Tilman, D., Wardle, D. a, Kinzig, A.P., Daily, G.C., Loreau, M., Grace, J.B., Larigauderie, A., Srivastava, D.S. \& Naeem, S. (2012) Biodiversity loss and its impact on humanity. Nature, 486, 59-67.

Cardinale, B.J., Gross, K., Fritschie, K., Flombaum, P., Fox, J.W., Rixen, C., Van Ruijven, J., Reich, P.B., Scherer-Lorenzen, M. \& Wilsey, B.J. (2013) Biodiversity simultaneously enhances the production and stability of community biomass, but the effects are independent. Ecology, 94, 1697-1707. 
Cardinale, B.J., Harvey, C.T., Gross, K. \& Ives, A.R. (2003) Biodiversity and biocontrol: emergent impacts of a multi-enemy assemblage on pest suppression and crop yield in an agroecosystem. Ecology Letters, 6, 857-865.

Cardinale, B.J., Srivastava, D.S., Duffy, J.E., Wright, J.P., Downing, A.L., Sankaran, M. \& Jouseau, C. (2006) Effects of biodiversity on the functioning of trophic groups and ecosystems. Nature, 443, 989-992.

Ceballos, G., Ehrlich, P.R., Barnosky, A.D., García, A., Pringle, R.M. \& Palmer, T.M. (2015) Accelerated modern human-induced species losses: entering the sixth mass extinction. Sciences Advances, 1, 1-5.

Chalcraft, D.R., Williams, J.W., Smith, M.D. \& Willig, M.R. (2004) Scale dependence in the species-richness-productivity relationship: the role of species turnover. Ecology, 85, 2701-2708.

Chao, A., Chazdon, R.L. \& Shen, T.J. (2005) A new statistical approach for assessing similarity of species composition with incidence and abundance data. Ecology Letters, 8, 148-159.

Chapin III, F.S., Zavaleta, E.S., Eviner, V.T., Naylor, R.L., Vitousek, P.M., Reynolds, H.L., Hooper, D.U., Lavorel, S., Sala, O.E., Hobbie, S.E., Mack, M.C. \& Diaz, S. (2000) Consequences of changing biodiversity. Nature, 405, 234-242.

Chave, J., Andalo, C., Brown, S., Cairns, M.A., Chambers, J.Q., Eamus, D., Fölster, H., Fromard, F., Higuchi, N., Kira, T., Lescure, J.P., Nelson, B.W., Ogawa, H., Puig, H., Riéra, B. \& Yamakura, T. (2005) Tree allometry and improved estimation of carbon stocks and balance in tropical forests. Oecologia, 145, 87-99.

Chen, I.-C., Hill, J.K., Ohlemüller, R., Roy, D.B. \& Thomas, C.D. (2011) Rapid range shifts of species associated with high levels of climate warming. Science, 333, 10246.

Colwell, R.K. (2013) EstimateS: statistical estimation of species richness and shared species from samples, In Version 9. User's Guide and application published at: http://purl.oclc.org/estimates.

Connell, J.H. (1978) Diversity in tropical rain forests and coral reefs. Science, 199, 13021310.

Corlatti, L., Hackländer, K. \& Frey-Roos, F. (2009) Ability of wildlife overpasses to provide connectivity and prevent genetic isolation. Conservation Biology, 23, 54856.

Davidson, E.A., Eckert, R.W., Hart, S.C. \& Firestone, M.K. (1989) Direct extraction of microbial biomass nitrogen from forest and grassland soils of California. Soil Biology \& Biochemistry, 21, 773-778. 
DeFries, R.S., Foley, J.A. \& Asner, G.P. (2004) Land-use choices: balancing human needs and ecosystem function. Frontiers in Ecology and the Environment, 2, 249257.

Denno, R.F., Gratton, C., Döbel, H. \& Finke, D.L. (2003) Predation risk affects relative strength of top-down and bottom-up impacts on insects herbivores. Ecology, 84, 1032-1044.

Denno, R.F., Gratton, C., Peterson, M.A., Langellotto, G.A., Finke, D.L. \& Huberty, A.F. (2002) Bottom-up forces mediate natural-enemy impact in a phytophagous insect community. Ecology, 83, 1443-1458.

Devictor, V., Julliard, R. \& Jiguet, F. (2008) Distribution of specialist and generalist species along spatial gradients of habitat disturbance and fragmentation. Oikos, 080211051304426 .

Díaz, S. \& Cabido, M. (2001) Vive la difference: plant functional diversity matters to ecosystem processes. Trends in Ecology \& Evolution, 16, 646-655.

Díaz, S., Fargione, J., Chapin, F.S. \& Tilman, D. (2006) Biodiversity loss threatens human well-being. PLoS biology, 4, e277.

Didham, R.K. \& Ewers, R.M. (2014) Edge effects disrupt vertical stratification of microclimate in a temperate forest canopy. Pacific Science, 68, 493-508.

Didham, R.K., Tylianakis, J.M., Gemmell, N.J., Rand, T.A. \& Ewers, R.M. (2007) Interactive effects of habitat modification and species invasion on native species decline. Trends in Ecology \& Evolution, 22, 489-496.

Digel, C., Curtsdotter, A., Riede, J., Klarner, B. \& Brose, U. (2014) Unravelling the complex structure of forest soil food webs: higher omnivory and more trophic levels. Oikos, 123, 1157-1172.

Dirzo, R. \& Raven, P.H. (2003) Global state of biodiversity and loss. Annual Review of Environment and Resources, 28, 137-167.

Dobson, A., Lodge, D., Alder, J., Cumming, G.S., Keymer, J., McGlade, J., Mooney, H., Rusak, J.A., Sala, O., Wolters, V., Wall, D., Winfree, R. \& Xenopoulos, M.A. (2006) Habitat loss, trophic collapse, and the decline of ecosystem services. Ecology, 87, 1915-1924.

Dormann, C.F., McPherson, J.M., Araújo, M.B., Bivand, R., Bolliger, J., Carl, G., Davies, R.G., Hirzel, A., Jetz, W., Daniel Kissling, W., Kühn, I., Ohlemüller, R., Peres-Neto, P.R., Reineking, B., Schröder, B., Schurr, F.M. \& Wilson, R. (2007) Methods to account for spatial autocorrelation in the analysis of species distributional data: a review. Ecography, 30, 609-628.

Duffy, J.E. (2002) Biodiversity and ecosystem function: the consumer connection. Oikos, 99, 201-219. 
Duffy, J.E. (2009) Why biodiversity is important to the functioning of real-world ecosystems. Frontiers in Ecology and the Environment, 7, 437-444.

Duffy, J.E., Cardinale, B.J., France, K.E., McIntyre, P.B., Thébault, E. \& Loreau, M. (2007) The functional role of biodiversity in ecosystems: incorporating trophic complexity. Ecology Letters, 10, 522-38.

Ebeling, A., Meyer, S.T., Abbas, M., Eisenhauer, N., Hillebrand, H., Lange, M., Scherber, C., Vogel, A., Weigelt, A. \& Weisser, W.W. (2014) Plant diversity impacts decomposition and herbivory via changes in aboveground arthropods. PLoS ONE, 9, e106529.

Edgar, R.C., Haas, B.J., Clemente, J.C., Quince, C. \& Knight, R. (2011) UCHIME improves sensitivity and speed of chimera detection. Bioinformatics, 27, 2194-2200.

Edwards, F.A., Edwards, D.P., Larsen, T.H., Hsu, W.W., Benedick, S., Chung, A., Vun Khen, C., Wilcove, D.S. \& Hamer, K.C. (2014a) Does logging and forest conversion to oil palm agriculture alter functional diversity in a biodiversity hotspot? Animal Conservation, 17, 163-173.

Edwards, D.P., Tobias, J.A., Sheil, D., Meijaard, E. \& Laurance, W.F. (2014b) Maintaining ecosystem function and services in logged tropical forests. Trends in ecology \& evolution, 29, 511-520.

Eggleton, P., Vanbergen, A.J., Jones, D.T., Lambert, M.C., Rockett, C., Hammond, P.M., Beccaloni, J., Marriott, D., Ross, E. \& Giusti, A. (2005) Assemblages of soil macrofauna across a Scottish land-use intensification gradient: influences of habitat quality, heterogeneity and area. Journal of Applied Ecology, 42, 1153-1164.

Ehnes, R., Pollierer, M., Erdmann, G., Klarner, B., Eitzinger, B., Digel, C., Ott, D., Maraun, M., Scheu, S. \& Brose, U. (2014) Lack of energetic equivalence in forest soil invertebrates. Ecology, 95, 527-537.

Ehnes, R., Rall, B. \& Brose, U. (2011) Phylogenetic grouping, curvature and metabolic scaling in terrestrial invertebrates. Ecology Letters, 14, 993-1000.

Ehrlich, P.R. \& Ehrlich, A.H. (2013) Can a collapse of global civilization be avoided? Proc Biol Sci, 280, 20122845.

Ehrlich, P.R., Kareiva, P.M. \& Daily, G.C. (2012) Securing natural capital and expanding equity to rescale civilization. Nature, 486, 68-73.

Eisenhauer, N., Fisichelli, N.A., Frelich, L.E. \& Reich, P.B. (2012) Interactive effects of global warming and "global worming" on the initial establishment of native and exotic herbaceous plant species. Oikos, 121, 1121-1133.

Elmqvist, T., Folke, C., Nyström, M., Peterson, G., Bengtsson, J., Walker, B. \& Norberg, J. (2003) Response diversity, ecosystem change, and resilience. Frontiers in Ecology and the Environment, 1, 488-494. 
Elton, C.S. (1958) The Ecology of Invasions by Animals and Plants. Springer US, Boston, MA.

Emmerson, M.C., Solan, M., Emes, C., Paterson, D.M. \& Raffaelli, D. (2001) Consistent patterns and the idiosyncratic effects of biodiversity in marine ecosystems. Nature, 411, 73-77.

Enquist, B.J., Kerkhoff, A.J., Stark, S.C., Swenson, N.G., McCarthy, M.C. \& Price, C.A. (2007) A general integrative model for scaling plant growth, carbon flux, and functional trait spectra. Nature, 449, 218-22.

Ewers, R.M. \& Didham, R.K. (2006) Confounding factors in the detection of species responses to habitat fragmentation. Biological Reviews, 81, 117-142.

Fahrig, L. (2003) Effects of habitat fragmentation on biodiversity. Annual Review of Ecology, Evolution, and Systematics, 34, 487-515.

Fahrig, L., Baudry, J., Brotons, L., Burel, F.G., Crist, T.O., Fuller, R.J., Sirami, C., Siriwardena, G.M. \& Martin, J.L. (2011) Functional landscape heterogeneity and animal biodiversity in agricultural landscapes. Ecology Letters, 14, 101-112.

FAO, IIASA, ISRIC, ISSCAS \& JRC. Harmonized World Soil Database (version 1.1). URL http://www.fao.org/soils-portal/soil-survey/soil-maps-anddatabases/harmonized-world-soil-database-v12/en/ [accessed 8 December 2014]

Fayle, T.M., Yusah, K.M. \& Hashimoto, Y. (2014) Key to the ant genera of Borneo in English and Malay.

Fischer, J., Lindenmayer, D.B., Blomberg, S.P., Montague-Drake, R., Felton, A. \& Stein, J.A. (2007) Functional richness and relative resilience of bird communities in regions with different land use intensities. Ecosystems, 10, 964-974.

Fitzherbert, E.B., Struebig, M.J., Morel, A., Danielsen, F., Brühl, C.A., Donald, P.F. \& Phalan, B. (2008) How will oil palm expansion affect biodiversity? Trends in ecology \& evolution, 23, 538-45.

Flynn, D.F.B., Gogol-Prokurat, M., Nogeire, T., Molinari, N., Richers, B.T., Lin, B.B., Simpson, N., Mayfield, M.M. \& DeClerck, F. (2009) Loss of functional diversity under land use intensification across multiple taxa. Ecology letters, 12, 22-33.

Flynn, D.F.B., Mirotchnick, N., Jain, M., Palmer, M.I. \& Naeem, S. (2011) Functional and phylogenetic diversity as predictors of biodiversity--ecosystem-function relationships. Ecology, 92, 1573-81.

Foley, J.A., DeFries, R., Asner, G.P., Barford, C., Bonan, G., Carpenter, S.R., Chapin, F.S., Coe, M.T., Daily, G.C., Gibbs, H.K., Helkowski, J.H., Holloway, T., Howard, E.A., Kucharik, C.J., Monfreda, C., Patz, J.A., Prentice, I.C., Ramankutty, N. \& Snyder, P.K. (2005) Global consequences of land use. Science, 309, 570-574. 
Folke, C., Carpenter, S., Walker, B., Scheffer, M., Elmqvist, T., Gunderson, L. \& Holling, C.S. (2004) Regime shifts, resilience, and biodiversity in ecosystem management. Annual Review of Ecology, Evolution, and Systematics, 35, 557-581.

Fonseca, C.R. \& Ganade, G. (2001) Species functional redundancy, random extinctions and the stability of ecosystems. Journal of Ecology, 89, 118-125.

Foster, W.A., Snaddon, J.L., Turner, E.C., Fayle, T.M., Cockerill, T.D., Ellwood, M.D.F., Broad, G.R., Chung, A.Y.C., Eggleton, P., Khen, C.V. \& Yusah, K.M. (2011) Establishing the evidence base for maintaining biodiversity and ecosystem function in the oil palm landscapes of South East Asia. Philosophical Transactions of the Royal Society B: Biological Sciences, 366, 3277-91.

France, K.E. \& Duffy, J.E. (2006) Diversity and dispersal interactively affect predictability of ecosystem function. Nature, 441, 1139-1143.

Frank, K.T., Petrie, B., Choi, J.S. \& Leggett, W.C. (2005) Trophic cascades in a formerly cod-dominated ecosystem. Science, 308, 1621-1623.

Frost, C.M., Didham, R.K., Rand, T.A., Peralta, G. \& Tylianakis, J.M. (2014) Community-level net spillover of natural enemies from managed to natural forest. Ecology, 96, 193-202.

Fukami, T., Martijn Bezemer, T., Mortimer, S.R. \& van der Putten, W.H. (2005) Species divergence and trait convergence in experimental plant community assembly. Ecology Letters, 8, 1283-1290.

Gabriel, D., Sait, S.M., Hodgson, J.A., Schmutz, U., Kunin, W.E. \& Benton, T.G. (2010) Scale matters: the impact of organic farming on biodiversity at different spatial scales. Ecology Letters, 13, 858-869.

Gagic, V., Bartomeus, I., Jonsson, T., Taylor, A., Winqvist, C., Fischer, C., Slade, E.M., Steffan-Dewenter, I., Emmerson, M., Potts, S.G., Tscharntke, T., Weisser, W. \& Bommarco, R. (2015) Functional identity and diversity of animals predict ecosystem functioning better than species-based indices. Proceedings of the Royal Society of London B: Biological Sciences, 282.

Gamfeldt, L., Hillebrand, H. \& Jonsson, P.R. (2005) Species richness changes across two trophic levels simultaneously affect prey and consumer biomass. Ecology Letters, $\mathbf{8}$, 696-703.

Gardner, T.A., Barlow, J., Chazdon, R., Ewers, R.M., Harvey, C.A., Peres, C.A. \& Sodhi, N.S. (2009) Prospects for tropical forest biodiversity in a human-modified world. Ecology Letters, 12, 561-582.

Gaveau, D., Wandono, H. \& Setiabudi, F. (2007) Three decades of deforestation in southwest Sumatra: have protected areas halted forest loss and logging, and promoted re-growth? Biological Conservation, 134, 495-504. 
Gee, G. \& Bauder, J. (1986) Particle-size analysis. Methods of soil analysis, Part 1 (ed A. Klute), pp. 383-411. Soil Science Society of America, Madison, WI.

Geiger, F., Bengtsson, J., Berendse, F., Weisser, W.W., Emmerson, M., Morales, M.B., Ceryngier, P., Liira, J., Tscharntke, T., Winqvist, C., Eggers, S., Bommarco, R., Pärt, T., Bretagnolle, V., Plantegenest, M., Clement, L.W., Dennis, C., Palmer, C., Oñate, J.J., Guerrero, I., Hawro, V., Aavik, T., Thies, C., Flohre, A., Hänke, S., Fischer, C., Goedhart, P.W. \& Inchausti, P. (2010) Persistent negative effects of pesticides on biodiversity and biological control potential on European farmland. Basic and Applied Ecology, 11, 97-105.

Gelman, A. (2008) Scaling regression inputs by dividing by two standard deviations. Statistics in Medicine, 27, 2865-2873.

Gibbs, H.K., Ruesch, A.S., Achard, F., Clayton, M.K., Holmgren, P., Ramankutty, N. \& Foley, J.A. (2010) Tropical forests were the primary sources of new agricultural land in the 1980s and 1990s. Proceedings of the National Academy of Sciences of the United States of America, 107, 16732-7.

Gibson, L., Lee, T.M., Koh, L.P., Brook, B.W., Gardner, T.A., Barlow, J., Peres, C.A., Bradshaw, C.J.A., Laurance, W.F., Lovejoy, T.E. \& Sodhi, N.S. (2011) Primary forests are irreplaceable for sustaining tropical biodiversity. Nature, 478, 378-381.

Gilbert, N. (2012) Palm-oil boom raises conservation concerns. Nature, 487, 14-5.

Gillison, A., Jones, D., Susilo, F. \& Bignell, D. (2003) Vegetation indicates diversity of soil macroinvertebrates: a case study with termites along a land-use intensification gradient in lowland Sumatra. Organisms Diversity \& Evolution, 3, 111-126.

Godbold, J.A., Bulling, M.T. \& Solan, M. (2011) Habitat structure mediates biodiversity effects on ecosystem properties. Proceedings of the Royal Society B: Biological Sciences, 278, 2510-2518.

Gonthier, D.J., Ennis, K.K., Farinas, S., Hsieh, H.-Y., Iverson, A.L., Batary, P., Rudolphi, J., Tscharntke, T., Cardinale, B.J. \& Perfecto, I. (2014) Biodiversity conservation in agriculture requires a multi-scale approach. Proceedings of the Royal Society B: Biological Sciences, 281, 20141358-20141358.

Gouyon, A., Foresta, H. \& Levang, P. (1993) Does jungle rubber deserve its name? An analysis of rubber agroforestry systems in southeast Sumatra. Agroforestry Systems, 22, 181-206.

Gowing, G. \& Recher, H.F. (1984) Length-weight relationships for invertebrates from forests in south-eastern New South Wales. Austral Ecology, 9, 5-8.

Grace, J.B. (2006) Structural Equation Modeling and Natural Systems. Cambridge University Press. 
Gray, C.L., Slade, E.M., Mann, D.J. \& Lewis, O.T. (2014) Do riparian reserves support dung beetle biodiversity and ecosystem services in oil palm-dominated tropical landscapes? Ecology and Evolution, 4, 1049-1060.

Grove, S.J. (2001) Extent and composition of dead wood in Australian lowland tropical rainforest with different management histories. Forest Ecology and Management, 154, 35-53.

Haddad, N.M., Crutsinger, G.M., Gross, K., Haarstad, J., Knops, J.M.H. \& Tilman, D. (2009) Plant species loss decreases arthropod diversity and shifts trophic structure. Ecology Letters, 12, 1029-1039.

Handa, I.T., Aerts, R., Berendse, F., Berg, M.P., Bruder, A., Butenschoen, O., Chauvet, E., Gessner, M.O., Jabiol, J., Makkonen, M., McKie, B.G., Malmqvist, B., Peeters, E.T.H.M., Scheu, S., Schmid, B., van Ruijven, J., Vos, V.C.A. \& Hättenschwiler, S. (2014) Consequences of biodiversity loss for litter decomposition across biomes. Nature, 509, 218-21.

Hautier, Y., Niklaus, P.A. \& Hector, A. (2009) Competition for light causes plant biodiversity loss after eutrophication. Science, 324, 636-638.

Hector, A. \& Bagchi, R. (2007) Biodiversity and ecosystem multifunctionality. Nature, 448, 188-90.

Hoehn, P., Tscharntke, T., Tylianakis, J.M. \& Steffan-Dewenter, I. (2008) Functional group diversity of bee pollinators increases crop yield. Proceedings of the Royal Society B: Biological Sciences, 275, 2283-2291.

Hof, C., Levinsky, I., Araújo, M.B. \& Rahbek, C. (2011) Rethinking species' ability to cope with rapid climate change. Global Change Biology, 17, 2987-2990.

Hooper, D.U., Chapin, F.S., Ewel, J.J., Hector, A., Inchausti, P., Lavorel, S., Lawton, J.H., Lodge, D.M., Loreau, M., Naeem, S., Schmid, B., Setala, H., Symstad, A.J., Vandermeer, J. \& Wardle, D.A. (2005) Effects of biodiversity on ecosystem functioning: a consensus of current knowledge. Ecological Monographs, 75, 3-35.

Hunter, M.D., Varley, G.C. \& Gradwell, G.R. (1997) Estimating the relative roles of topdown and bottom-up forces on insect herbivore populations: a classic study revisited. Proceedings of the National Academy of Sciences of the United States of America, 94, 9176-9181.

Huston, M.A. (1997) Hidden treatments in ecological experiments: re-evaluating the ecosystem function of biodiversity. Oecologia, 110, 449-460.

Isaacs, R., Tuell, J., Fiedler, A., Gardiner, M. \& Landis, D. (2009) Maximizing arthropodmediated ecosystem services in agricultural landscapes: the role of native plants. Frontiers in Ecology and the Environment, 7, 196-203. 
Isbell, F.I., Polley, H.W. \& Wilsey, B.J. (2009) Biodiversity, productivity and the temporal stability of productivity: patterns and processes. Ecology Letters, 12, 443451.

Ives, A.R., Cardinale, B.J. \& Snyder, W.E. (2004) A synthesis of subdisciplines: predator-prey interactions, and biodiversity and ecosystem functioning. Ecology Letters, 8, 102-116.

Janzen, D.H. (1974) The deflowering of Central America. Natural History, 83, 48-53.

Jentsch, A., Kreyling, J., \& Beierkuhnlein, C. (2007) A new generation of climate change experiments: events, not trends. Frontiers in Ecology and the Environment, 5, 365374.

Jochum, M., Schneider, F.D., Crowe, T.P., Brose, U. \& O'Gorman, E.J. (2012) Climateinduced changes in bottom-up and top-down processes independently alter a marine ecosystem. Philosophical Transactions of the Royal Society B: Biological Sciences, 367, 2962-70.

Jones, D.T., Susilo, F.X., Bignell, D.E., Hardiwinoto, S., Gillison, A.N. \& Eggleton, P. (2003) Termite assemblage collapse along a land-use intensification gradient in lowland central Sumatra, Indonesia. Journal of Applied Ecology, 40, 380-391.

Kagata, H. \& Ohgushi, T. (2006) Bottom-up trophic cascades and material transfer in terrestrial food webs. Ecological Research, 21, 26-34.

Karp, D.S., Ziv, G., Zook, J., Ehrlich, P.R. \& Daily, G.C. (2011) Resilience and stability in bird guilds across tropical countryside. Proceedings of the National Academy of Sciences of the United States of America, 108, 21134-21139.

Kaspari, M. \& Yanoviak, S.P. (2009) Biogeochemistry and the structure of tropical brown food webs. Ecology, 90, 3342-3351.

Kauffman, J.B. \& Donato, D. (2012) Protocols for the Measurement, Monitoring and Reporting of Structure, Biomass and Carbon Stocks in Mangrove Forests. Center for International Forestry Research (CIFOR), Bogor, Indonesia.

Klarner, B., Ehnes, R.B., Erdmann, G., Eitzinger, B., Pollierer, M.M., Maraun, M. \& Scheu, S. (2014) Trophic shift of soil animal species with forest type as indicated by stable isotope analysis. Oikos, 123, 1173-1181.

van Kleunen, M., Dawson, W., Essl, F., Pergl, J., Winter, M., Weber, E., Kreft, H., Weigelt, P., Kartesz, J., Nishino, M., Antonova, L.A., Barcelona, J.F., Cabezas, F.J., Cárdenas, D., Cárdenas-Toro, J., Castaño, N., Chacón, E., Chatelain, C., Ebel, A.L., Figueiredo, E., Fuentes, N., Groom, Q.J., Henderson, L., Inderjit, Kupriyanov, A., Masciadri, S., Meerman, J., Morozova, O., Moser, D., Nickrent, D.L., Patzelt, A., Pelser, P.B., Baptiste, M.P., Poopath, M., Schulze, M., Seebens, H., Shu, W., Thomas, J., Velayos, M., Wieringa, J.J. \& Pyšek, P. (2015) Global exchange and accumulation of non-native plants. Nature. 
Kneitel, J.M. \& Chase, J.M. (2004) Trade-offs in community ecology: Linking spatial scales and species coexistence. Ecology Letters, 7, 69-80.

Koh, L.P. \& Ghazoul, J. (2008) Biofuels, biodiversity, and people: understanding the conflicts and finding opportunities. Biological Conservation, 141, 2450-2460.

Koh, L.P. \& Ghazoul, J. (2010) Spatially explicit scenario analysis for reconciling agricultural expansion, forest protection, and carbon conservation in Indonesia.

Proceedings of the National Academy of Sciences of the United States of America, 107, 11140-4.

Koh, L.P., Miettinen, J., Liew, S.C. \& Ghazoul, J. (2011) Remotely sensed evidence of tropical peatland conversion to oil palm. Proceedings of the National Academy of Sciences of the United States of America, 108, 5127-32.

Koh, L.P. \& Wilcove, D.S. (2007) Cashing in palm oil for conservation. Nature, 448, 993-994.

Koh, L.P. \& Wilcove, D.S. (2008) Is oil palm agriculture really destroying tropical biodiversity? Conservation Letters, 1, 60-64.

Kotowska, M.M., Leuschner, C., Triadiati, T., Meriem, S. \& Hertel, D. (2015) Quantifying above- and belowground biomass carbon loss with forest conversion in tropical lowlands of Sumatra (Indonesia). Global Change Biology, 21, 3620-3634.

Kremen, C., Williams, N.M., Aizen, M.A., Gemmill-Herren, B., LeBuhn, G., Minckley, R., Packer, L., Potts, S.G., Roulston, T., Steffan-Dewenter, I., Vázquez, D.P., Winfree, R., Adams, L., Crone, E.E., Greenleaf, S.S., Keitt, T.H., Klein, A.-M., Regetz, J. \& Ricketts, T.H. (2007) Pollination and other ecosystem services produced by mobile organisms: a conceptual framework for the effects of land-use change. Ecology Letters, 10, 299-314.

Kupfer, J.A., Malanson, G.P. \& Franklin, S.B. (2006) Not seeing the ocean for the islands: the mediating influence of matrix-based processes on forest fragmentation effects. Global Ecology \& Biogeography, 15, 8-20.

Laliberté, E. \& Legendre, P. (2010) A distance-based framework for measuring functional diversity from multiple traits. Ecology, 91, 299-305.

Laliberté, E. \& Tylianakis, J.M. (2012) Cascading effects of long-term land-use changes on plant traits and ecosystem functioning. Ecology, 93, 145-55.

Laliberté, E., Wells, J.A., DeClerck, F., Metcalfe, D.J., Catterall, C.P., Queiroz, C., Aubin, I., Bonser, S.P., Ding, Y., Fraterrigo, J.M., McNamara, S., Morgan, J.W., Merlos, D.S., Vesk, P.A. \& Mayfield, M.M. (2010) Land-use intensification reduces functional redundancy and response diversity in plant communities. Ecology Letters, 13, 76-86. 
Lambin, E.F., Geist, H.J. \& Lepers, E. (2003) Dynamics of land-use and land-cover change in tropical regions. Annual Review of Environment and Resources, 28, 205241.

Lambin, E.F. \& Meyfroidt, P. (2010) Land use transitions: socio-ecological feedback versus socio-economic change. Land Use Policy, 27, 108-118.

Lambin, E.F. \& Meyfroidt, P. (2011) Global land use change, economic globalization, and the looming land scarcity. Proceedings of the National Academy of Sciences of the United States of America, 108, 3465-3472.

Lang, B., Rall, B.C., Scheu, S. \& Brose, U. (2014) Effects of environmental warming and drought on size-structured soil food webs. Oikos, 123, 1224-1233.

Lanta, V. \& Lepš, J. (2007) Effects of species and functional group richness on production in two fertility environments: an experiment with communities of perennial plants. Acta Oecologica, 32, 93-103.

Larsen, T.H., Lopera, A. \& Forsyth, A. (2008) Understanding trait-dependent community disassembly: dung beetles, density functions, and forest fragmentation. Conservation Biology, 22, 1288-1298.

Larsen, N., Olsen, G.J., Maidak, B.L., McCaughey, M.J., Overbeek, R., Macke, T.J., Marsh, T.L. \& Woese, C.R. (1993) The ribosomal database project. Nucleic acids research, 21, 3021-3023.

Larsen, T.H., Williams, N.M. \& Kremen, C. (2005) Extinction order and altered community structure rapidly disrupt ecosystem functioning. Ecology Letters, 8, 538547.

Laumonier, Y., Uryu, Y., Stüwe, M., Budiman, A., Setiabudi, B. \& Hadian, O. (2010) Eco-floristic sectors and deforestation threats in Sumatra: identifying new conservation area network priorities for ecosystem-based land use planning. Biodiversity and Conservation, 19, 1153-1174.

Laurance, W.F., Ferreira, L.V., Merona, J.M.R., Laurance, S.G., Hutchings, R.W. \& Lovejoy, T.E. (1998) Effects of forest fragmentation on recruitment patterns in Amazonian tree communities. Conservation Biology, 12, 460-464.

Laurance, W.F., Sayer, J. \& Cassman, K.G. (2014) Agricultural expansion and its impacts on tropical nature. Trends in ecology \& evolution, 29, 107-16.

Lawton, J.H., Bignell, D.E., Bolton, B., Bloemers, G.F., Eggleton, P., Hammond, P.M., Hodda, M., Holt, R.D., Larsen, T.B., Mawdsley, N.A., Stork, N.E., Srivastava, D.S. \& Watt, A.D. (1998) Biodiversity inventories, indicator taxa and effects of habitat modification in tropical forest. Nature, 391, 72-76.

Lefcheck, J.S., Byrnes, J.E.K., Isbell, F., Gamfeldt, L., Griffin, J.N., Eisenhauer, N., Hensel, M.J.S., Hector, A., Cardinale, B.J. \& Duffy, J.E. (2015) Biodiversity 
enhances ecosystem multifunctionality across trophic levels and habitats. Nat Commun, 6.

Lefcheck, J.S. \& Duffy, J.E. (2015) Multitrophic functional diversity predicts ecosystem functioning in experimental assemblages of estuarine consumers. Ecology.

Li, H., Aide, T.M., Ma, Y., Liu, W. \& Cao, M. (2007) Demand for rubber is causing the loss of high diversity rain forest in SW China. Biodiversity and Conservation, 16, 1731-1745.

Lichstein, J.W. (2007) Multiple regression on distance matrices: a multivariate spatial analysis tool. Plant Ecology, 188, 117-131.

Lucey, J.M. \& Hill, J.K. (2012) Spillover of insects from rain forest into adjacent oil palm plantations. Biotropica, 44, 368-377.

Luck, G.W., Carter, A. \& Smallbone, L. (2013) Changes in bird functional diversity across multiple land uses: interpretations of functional redundancy depend on functional group identity. PloS one, 8, e63671.

MacDougall, A.S., McCann, K.S., Gellner, G. \& Turkington, R. (2013) Diversity loss with persistent human disturbance increases vulnerability to ecosystem collapse. Nature, 494, 86-89.

Mäder, P., Fliessbach, A., Dubois, D., Gunst, L., Fried, P. \& Niggli, U. (2002) Soil fertility and biodiversity in organic farming. Science, 296, 1694-1697.

Maire, E., Grenouillet, G., Brosse, S. \& Villéger, S. (2015) How many dimensions are needed to accurately assess functional diversity? A pragmatic approach for assessing the quality of functional spaces. Global Ecology and Biogeography, 24, 728-740.

Margono, B., Potapov, P., Turubanova, S., Stolle, F. \& Hansen, M.C. (2014) Primary forest cover loss in Indonesia over 2000-2012. Nature Climate Change, 4, 730-735.

Matson, P.A., Parton, W.J., Power, A.G. \& Swift, M.J. (1997) Agricultural intensification and ecosystem properties. Science, 277, 504-509.

McGill, B.J., Enquist, B.J., Weiher, E. \& Westoby, M. (2006) Rebuilding community ecology from functional traits. Trends in Ecology \& Evolution, 21, 178-185.

Mori, A.S., Furukawa, T. \& Sasaki, T. (2013) Response diversity determines the resilience of ecosystems to environmental change. Biological Reviews, 88, 349-364.

Mouchet, M.A., Villéger, S., Mason, N.W.H. \& Mouillot, D. (2010) Functional diversity measures: an overview of their redundancy and their ability to discriminate community assembly rules. Functional Ecology, 24, 867-876.

Murdiyarso, D., Van Noordwijk, M., Wasrin, U.R., Tomich, T.P. \& Gillison, A.N. (2002) Environmental benefits and sustainable land-use options in the Jambi transect, Sumatra. Journal of Vegetation Science, 13, 429-438. 
Murty, D., Kirschbaum, M.U.F., McMurtrie, R.E. \& McGilvray, H. (2002) Does conversion of forest to agricultural land change soil carbon and nitrogen? A review of the literature. Global Change Biology, 8, 105-123.

Myers, J.A., Chase, J.M., Jiménez, I., Jørgensen, P.M., Araujo-Murakami, A., PaniaguaZambrana, N. \& Seidel, R. (2013) Beta-diversity in temperate and tropical forests reflects dissimilar mechanisms of community assembly. Ecology letters, 16, 151-7.

Myers, N., Mittermeier, R.A., Mittermeier, C.G., da Fonseca, G.A. \& Kent, J. (2000) Biodiversity hotspots for conservation priorities. Nature, 403, 853-8.

Naeem, S., Thompson, L.J., Lawler, S.P., Lawton, J.H. \& Woodfin, R.M. (1994) Declining biodiversity can alter the performance of ecosystems. Nature, 368, 734737.

Naeem, S. \& Wright, J.P. (2003) Disentangling biodiversity effects on ecosystem functioning: deriving solutions to a seemingly insurmountable problem. Ecology Letters, 6, 567-579.

Nakagawa, S. \& Schielzeth, H. (2013) A general and simple method for obtaining R2 from generalized linear mixed-effects models. Methods in Ecology \& Evolution, 4, $133-142$.

Narwani, A. \& Mazumder, A. (2012) Bottom-up effects of species diversity on the functioning and stability of food webs. Journal of Animal Ecology, 81, 701-713.

Newbold, T., Hudson, L.N., Hill, S.L.L., Contu, S., Lysenko, I., Senior, R.A., Börger, L., Bennett, D.J., Choimes, A. \& Collen, B. (2015) Global effects of land use on local terrestrial biodiversity. Nature, 520, 45-50.

Niiyama, K., Kajimoto, T., Matsuura, Y., Yamashita, T., Matsuo, N., Yashiro, Y., Ripin, A., Kassim, A.R. \& Noor, N.S. (2010) Estimation of root biomass based on excavation of individual root systems in a primary dipterocarp forest in Pasoh Forest Reserve, Peninsular Malaysia. Journal of Tropical Ecology, 26, 271.

Öckinger, E., Schweiger, O., Crist, T.O., Debinski, D.M., Krauss, J., Kuussaari, M., Petersen, J.D., Pöyry, J., Settele, J., Summerville, K.S. \& Bommarco, R. (2010) Life-history traits predict species responses to habitat area and isolation: a crosscontinental synthesis. Ecology letters, 13, 969-79.

Ott, D., Digel, C., Klarner, B., Maraun, M., Pollierer, M., Rall, B.C., Scheu, S., Seelig, G. \& Brose, U. (2014a) Litter elemental stoichiometry and biomass densities of forest soil invertebrates. Oikos, 123, 1212-1223.

Ott, D., Digel, C., Rall, B.C., Maraun, M., Scheu, S. \& Brose, U. (2014b) Unifying elemental stoichiometry and metabolic theory in predicting species abundances. Ecology Letters, 17, 1247-1256. 
Otto, S.B., Berlow, E.L., Rank, N.E., Smiley, J. \& Brose, U. (2008) Predator diversity and identity drive interaction strength and trophic cascades in a food web. Ecology, 89, 134-44.

Pace, M., Cole, J., Carpenter, S. \& Kitchell, J. (1999) Trophic cascades revealed in diverse ecosystems. Trends in ecology \& evolution, 14, 483-488.

Paine, R.T. (1980) Food webs: linkage, interaction strength and community infrastructure. Journal of Animal Ecology, 49, 667-685.

Pakeman, R.J. (2011) Functional diversity indices reveal the impacts of land use intensification on plant community assembly. Journal of Ecology, 99, 1143-1151.

Pereira, H.M., Leadley, P.W., Proença, V., Alkemade, R., Scharlemann, J.P.W., Fernandez-Manjarrés, J.F., Araújo, M.B., Balvanera, P., Biggs, R., Cheung, W.W.L., Chini, L., Cooper, H.D., Gilman, E.L., Guénette, S., Hurtt, G.C., Huntington, H.P., Mace, G.M., Oberdorff, T., Revenga, C., Rodrigues, P., Scholes, R.J., Sumaila, U.R. $\&$ Walpole, M. (2010) Scenarios for global biodiversity in the 21st century. Science, 330, 1496-1501.

Petchey, O.L., Downing, A.L., Mittelbach, G.G., Persson, L., Steiner, C.F., Warren, P.H. \& Woodward, G. (2004) Species loss and the structure and functioning of multitrophic aquatic systems. Oikos, 104, 467-478.

Petchey, O.L. \& Gaston, K.J. (2006) Functional diversity: back to basics and looking forward. Ecology letters, 9, 741-58.

Peters, R.H. (1983) The Ecological Implications of Body Size. Cambridge University Press, Cambridge.

Pianka, E.R. (1966) Latitudinal gradients in species diversity: a review of concepts. American Naturalist, 100, 33-46.

Pillar, V.D., Blanco, C.C., Müller, S.C., Sosinski, E.E., Joner, F. \& Duarte, L.D.S. (2013) Functional redundancy and stability in plant communities. Journal of Vegetation Science, 24, 963-974.

Pimm, S.L., Jenkins, C.N., Abell, R., Brooks, T.M., Gittleman, J.L., Joppa, L.N., Raven, P.H., Roberts, C.M. \& Sexton, J.O. (2014) The biodiversity of species and their rates of extinction, distribution, and protection. Science, 344, 1246752.

Poisot, T., Mouquet, N. \& Gravel, D. (2013) Trophic complementarity drives the biodiversity-ecosystem functioning relationship in food webs. Ecology Letters, 16, 853-861.

Potts, S.G., Biesmeijer, J.C., Kremen, C., Neumann, P., Schweiger, O. \& Kunin, W.E. (2010) Global pollinator declines: trends, impacts and drivers. Trends in Ecology and Evolution, 25, 345-353. 
Purvis, A., Gittleman, J.L., Cowlishaw, G. \& Mace, G.M. (2000) Predicting extinction risk in declining species. Proceedings. Biological sciences / The Royal Society, 267, 1947-52.

Quast, C., Pruesse, E., Yilmaz, P., Gerken, J., Schweer, T., Yarza, P., Peplies, J. \& Glöckner, F.O. (2012) The SILVA ribosomal RNA gene database project: improved data processing and web-based tools. Nucleic acids research, gks1219.

R Core Team. (2014) R: a language and environment for statistical computing. http://www.R-project.org/.

R Core Team. (2015) R: a language and environment for statistical computing. http://www.R-project.org/.

Rader, R., Bartomeus, I., Tylianakis, J.M. \& Laliberté, E. (2014) The winners and losers of land use intensification: pollinator community disassembly is non-random and alters functional diversity. Diversity and Distributions, 20, 908-917.

Rall, B.C., Kalinkat, G., Ott, D., Vucic-Pestic, O. \& Brose, U. (2011) Taxonomic versus allometric constraints on non-linear interaction strengths. Oikos, 120, 483-492.

Rand, T.A., Tylianakis, J.M. \& Tscharntke, T. (2006) Spillover edge effects: the dispersal of agriculturally subsidized insect natural enemies into adjacent natural habitats. Ecology Letters, 9, 603-614.

Reiss, J., Bridle, J.R., Montoya, J.M. \& Woodward, G. (2009) Emerging horizons in biodiversity and ecosystem functioning research. Trends in Ecology \& Evolution, 24, 505-514.

Rosenfeld, J.S. (2002) Logical fallacies in the assessment of functional redundancy. Conservation Biology, 16, 837-839.

De Ruiter, P.C., Neutel, a M. \& Moore, J.C. (1994) Modelling food webs and nutrient cycling in agro-ecosystems. Trends in ecology \& evolution, 9, 378-83.

De Ruiter, P.C., Veen, J. a., Moore, J.C., Brussaard, L. \& Hunt, H.W. (1993) Calculation of nitrogen mineralization in soil food webs. Plant and Soil, 157, 263-273.

Ryall, K. \& Fahrig, L. (2006) Response of predators to loss and fragmentation of prey habitat: a review of theory. Ecology, 87, 1086-1093.

Rybicki, J. \& Hanski, I. (2013) Species-area relationships and extinctions caused by habitat loss and fragmentation. Ecology Letters, 16, 27-38.

Sala, O.E., Chapin, F.S., Armesto, J.J., Berlow, E., Bloomfield, J., Dirzo, R., HuberSanwald, E., Huenneke, L.F., Jackson, R.B., Kinzig, A., Leemans, R., Lodge, D.M., Mooney, H.A., Oesterheld, M., Poff, N.L., Sykes, M.T., Walker, B.H., Walker, M. \& Wall, D.H. (2000) Global biodiversity scenarios for the year 2100. Science, 287, 1770-1774. 
Salamon, J.-A., Alphei, J., Ruf, A., Schaefer, M., Scheu, S., Schneider, K., Sührig, A. \& Maraun, M. (2006) Transitory dynamic effects in the soil invertebrate community in a temperate deciduous forest: Effects of resource quality. Soil Biology \& Biochemistry, 38, 209-221.

Scherber, C., Eisenhauer, N., Weisser, W.W., Schmid, B., Voigt, W., Fischer, M., Schulze, E.-D., Roscher, C., Weigelt, A., Allan, E., Bessler, H., Bonkowski, M., Buchmann, N., Buscot, F., Clement, L.W., Ebeling, A., Engels, C., Halle, S., Kertscher, I., Klein, A.-M., Koller, R., König, S., Kowalski, E., Kummer, V., Kuu, A., Lange, M., Lauterbach, D., Middelhoff, C., Migunova, V.D., Milcu, A., Müller, R., Partsch, S., Petermann, J.S., Renker, C., Rottstock, T., Sabais, A., Scheu, S., Schumacher, J., Temperton, V.M. \& Tscharntke, T. (2010) Bottom-up effects of plant diversity on multitrophic interactions in a biodiversity experiment. Nature, 468, 553-556.

Schneider, D., Arp, G., Reimer, A., Reitner, J. \& Daniel, R. (2013) Phylogenetic analysis of a microbialite-forming microbial mat from a hypersaline lake of the Kiritimati Atoll, Central Pacific. PLoS ONE, 8.

Schneider, F.D. \& Brose, U. (2013) Beyond diversity: how nested predator effects control ecosystem functions. Journal of Animal Ecology, 82, 64-71.

Schneider, F.D., Scheu, S. \& Brose, U. (2012) Body mass constraints on feeding rates determine the consequences of predator loss. Ecology Letters, 15, 436-43.

Schnitzer, S. a., DeWalt, S.J. \& Chave, J. (2006) Censusing and measuring lianas: a quantitative comparison of the common methods. Biotropica, 38, 581-591.

Schuster, E. \& Schröder, D. (1990) Side-effects of sequentially- and simultaneouslyapplied pesticides on non-target soil microorganisms: laboratory experiments. Soil Biology and Biochemistry, 22, 375-383.

Senior, M.J.M., Hamer, K.C., Bottrell, S., Edwards, D.P., Fayle, T.M., Lucey, J.M., Mayhew, P.J., Newton, R., Peh, K.S.-H., Sheldon, F.H., Stewart, C., Styring, A.R., Thom, M.D.F., Woodcock, P. \& Hill, J.K. (2012) Trait-dependent declines of species following conversion of rain forest to oil palm plantations. Biodiversity and Conservation, 22, 253-268.

Sheil, D., Casson, A., Meijaard, E., Nordwijk, V.M., Gaskell, J., Sunderland-Groves, J., Wertz, K. \& Kanninen, M. (2009) The impacts and opportunities of oil palm in Southeast Asia: what do we know and what do we need to know? CIFOR Occasional paper no. 51 .

Shipley, B. (2009) Confirmatory path analysis in a generalized multilevel context. Ecology, 90, 363-368.

Sodhi, N.S., Koh, L.P., Brook, B.W. \& Ng, P.K.L. (2004) Southeast Asian biodiversity: an impending disaster. Trends in ecology \& evolution, 19, 654-60. 
Soininen, J., Lennon, J.J. \& Hillebrand, H. (2007) A multivariate analysis of beta diversity across organisms and environments. Ecology, 88, 2830-2838.

Srivastava, D.S. \& Vellend, M. (2005) Biodiversity-ecosystem function research: is it relevant to conservation? Annual Review of Ecology, Evolution, and Systematics, 36, 267-294.

Striebel, M., Behl, S., Diehl, S. \& Stibor, H. (2009) Spectral niche complementarity and carbon dynamics in pelagic ecosystems. The American Naturalist, 174, 141-147.

Suding, K.N., Lavorel, S., Chapin, F.S., Cornelissen, J.H.C., Diaz, S., Garnier, E., Goldberg, D., Hooper, D.U., Jackson, S.T. \& Navas, M.-L. (2008) Scaling environmental change through the community-level: a trait-based response-andeffect framework for plants. Global Change Biology, 14, 1125-1140.

Swaine, M.D. (1996) Rainfall and soil fertility as factors limiting forest species distributions in Ghana. Journal of Ecology, 84, 419-428.

Syahrinudin. (2005) The potential of oil palm and forest plantations for carbon sequestration on degraded land in Indonesia. Ecology and Development Series No. 28 Cuvillier Verlag, Göttingen.

Symstad, A. (2000) A test of the effects of functional group richness and composition on grassland invasibility. Ecology, 81, 99-109.

Symstad, A.J., Tilman, D., Willson, J. \& Knops, J.M.H. (1998) Species loss and ecosystem functioning: effects of species identity and community composition. Oikos, 81, 389-397.

Thomas, C.D., Cameron, A., Green, R.E., Bakkenes, M., Beaumont, L.J., Collingham, Y.C., Erasmus, B.F.N., De Siqueira, M.F., Grainger, A., Hannah, L., Hughes, L., Huntley, B., Van Jaarsveld, A.S., Midgley, G.F., Miles, L., Ortega-Huerta, M.A., Peterson, A.T., Phillips, O.L. \& Williams, S.E. (2004) Extinction risk from climate change. Nature, 427, 145-8.

Thompson, R.M., Brose, U., Dunne, J.A., Hall, R.O., Hladyz, S., Kitching, R.L., Martinez, N.D., Rantala, H., Romanuk, T.N., Stouffer, D.B. \& Tylianakis, J.M. (2012) Food webs: reconciling the structure and function of biodiversity. Trends in Ecology and Evolution, 27, 689-697.

Tilman, D., Cassman, K.G., Matson, P.A., Naylor, R. \& Polasky, S. (2002) Agricultural sustainability and intensive production practices. Nature, 418, 671-677.

Tilman, D., Isbell, F. \& Cowles, J.M. (2014) Biodiversity and ecosystem functioning. Annual Review of Ecology, Evolution, and Systematics, 45, 471.

Tilman, D., Knops, J., Wedin, D., Reich, P.B., Ritchie, M. \& Siemann, E. (1997a) The influence of functional diversity and composition on ecosystem processes. Science, 277, 1300-1302. 
Tilman, D., Reich, P.B. \& Knops, J.M.H. (2006) Biodiversity and ecosystem stability in a decade-long grassland experiment. Nature, 441, 629-632.

Tilman, D., Reich, P.B., Knops, J., Wedin, D., Mielke, T. \& Lehman, C. (2001) Diversity and productivity in a long-term grassland experiment. Science, 294, 843-845.

Tilman, D., Tilman, D., Lehman, C.L., Lehman, C.L., Thomson, K.T. \& Thomson, K.T. (1997b) Plant diversity and ecosystem productivity: theoretical considerations.

Proceedings of the National Academy of Sciences of the United States of America, 94, 1857-61.

Tilman, D., Wedin, D. \& Knops, J. (1996) Productivity and sustainability influenced by biodiversity in grassland ecosystems. Nature, 379, 718-720.

Tripathi, B., Kim, M., Singh, D., Lee-Cruz, L., Lai-Hoe, A., Ainuddin, A.N., Go, R., Rahim, R., Husni, M.H.A., Chun, J. \& Adams, J. (2012) Tropical soil bacterial communities in Malaysia: $\mathrm{pH}$ dominates in the equatorial tropics too. Microbial Ecology, 64, 474-484.

Tscharntke, T., Clough, Y., Wanger, T.C., Jackson, L., Motzke, I., Perfecto, I., Vandermeer, J. \& Whitbread, A. (2012a) Global food security, biodiversity conservation and the future of agricultural intensification. Biological Conservation, 151, 53-59.

Tscharntke, T., Tylianakis, J.M., Rand, T.A., Didham, R.K., Fahrig, L., Batáry, P., Bengtsson, J., Clough, Y., Crist, T.O., Dormann, C.F., Ewers, R.M., Fründ, J., Holt, R.D., Holzschuh, A., Klein, A.M., Kleijn, D., Kremen, C., Landis, D.A., Laurance, W., Lindenmayer, D., Scherber, C., Sodhi, N., Steffan-Dewenter, I., Thies, C., van der Putten, W.H. \& Westphal, C. (2012b) Landscape moderation of biodiversity patterns and processes - eight hypotheses. Biological Reviews, 87, 661-685.

Turner, E.C. \& Foster, W.A. (2008) The impact of forest conversion to oil palm on arthropod abundance and biomass in Sabah, Malaysia. Journal of Tropical Ecology, 25, 23.

Turner, E.C., Snaddon, J.L., Fayle, T.M. \& Foster, W.A. (2008) Oil palm research in context: identifying the need for biodiversity assessment. PLOS ONE, 3, 2-5.

Tylianakis, J.M. (2013) The global plight of pollinators. Science, 339, 1532-1533.

Tylianakis, J.M., Didham, R.K., Bascompte, J. \& Wardle, D.A. (2008a) Global change and species interactions in terrestrial ecosystems. Ecology Letters, 11, 1351-1363.

Tylianakis, J.M., Rand, T.A., Kahmen, A., Klein, A.M., Buchmann, N., Perner, J. \& Tscharntke, T. (2008b) Resource heterogeneity moderates the biodiversity-function relationship in real world ecosystems. PLoS Biology, 6, 0947-0956.

Vetter, D., Rücker, G. \& Storch, I. (2013) A meta-analysis of tropical forest edge effects on bird nest predation risk: edge effects in avian nest predation. Biological Conservation, 159, 382-395. 
Villéger, S., Mason, N.W.H. \& Mouillot, D. (2008) New multidimensional functional diversity indices for a multifaceted framework in functional ecology. Ecology, 89, 2290-2301.

Violle, C., Enquist, B.J., McGill, B.J., Jiang, L., Albert, C.H., Hulshof, C., Jung, V. \& Messier, J. (2012) The return of the variance: intraspecific variability in community ecology. Trends in ecology \& evolution, 27, 244-52.

Violle, C., Navas, M.-L., Vile, D., Kazakou, E., Fortunel, C., Hummel, I. \& Garnier, E. (2007) Let the concept of trait be functional! Oikos, 116, 882-892.

Vitousek, P.M., Mooney, H.A., Lubchenco, J. \& Melillo, J.M. (1997) Human domination of Earth's ecosystems. Science, 277, 494-499.

Wanger, T.C., Rauf, A. \& Schwarze, S. (2010) Pesticides and tropical biodiversity. Frontiers in Ecology and the Environment, 8, 178-179.

Wauters, J.B., Coudert, S., Grallien, E., Jonard, M. \& Ponette, Q. (2008) Carbon stock in rubber tree plantations in Western Ghana and Mato Grosso (Brazil). Forest Ecology and Management, 255, 2347-2361.

Wielgoss, A., Tscharntke, T., Buchori, D., Fiala, B. \& Clough, Y. (2010) Temperature and a dominant dolichoderine ant species affect ant diversity in Indonesian cacao plantations. Agriculture, ecosystems \& environment, 135, 253-259.

Wilcove, D.S., Giam, X., Edwards, D.P., Fisher, B. \& Koh, L.P. (2013) Navjot's nightmare revisited: logging, agriculture, and biodiversity in Southeast Asia. Trends in Ecology \& Evolution, 28, 531-40.

Wilcove, D.S. \& Koh, L.P. (2010) Addressing the threats to biodiversity from oil-palm agriculture. Biodiversity and Conservation, 19, 999-1007.

Wilman, H., Belmaker, J., Simpson, J., de la Rosa, C., Rivadeneira, M.M. \& Jetz, W. (2014) EltonTraits 1.0: Species-level foraging attributes of the world's birds and mammals. Ecology, 95, 2027.

Wilson, E.O. \& Hölldobler, B. (2005) Eusociality: origin and consequences. Proceedings of the National Academy of Sciences of the United States of America, 102, 1336713371.

Wootton, J.T. (1994) The nature and consequences of indirect effects in ecological communities. Annual Review of Ecology and Systematics, 25, 443-466.

Yachi, S. \& Loreau, M. (1999) Biodiversity and ecosystem productivity in a fluctuating environment: the insurance hypothesis. Proceedings of the National Academy of Sciences of the United States of America, 96, 1463-1468.

Zalasiewicz, J., Williams, M., Haywood, A. \& Ellis, M. (2011) The Anthropocene: a new epoch of geological time? Philosophical Transactions of the Royal Society A: Mathematical, physical, and engineering sciences, 369, 835-841. 
Zhang, B., Bai, Z., Hoefel, D., Tang, L., Wang, X., Li, B., Li, Z. \& Zhuang, G. (2009) The impacts of cypermethrin pesticide application on the non-target microbial community of the pepper plant phyllosphere. Science of the Total Environment, 407, 1915-1922. 


\section{SUPPLEMENTARY INFORMATION - CHAPTER 2}

Supplementary Figure 2.1 Map of the study region with an overview of Sumatra (a) and Jambi Province (b) with red and blue points denoting the 16 sites in Bukit Duabelas landscape and the 16 sites in Harapan landscape, respectively. Additionally, the spatial layout of the sampling sites in Bukit Duabelas landscape (c) and Harapan landscape (d) is represented by coloured crosses for forest $(\mathrm{F})$, jungle rubber $(\mathrm{J})$, rubber $(\mathrm{R})$ and oil palm $(\mathrm{O})$.

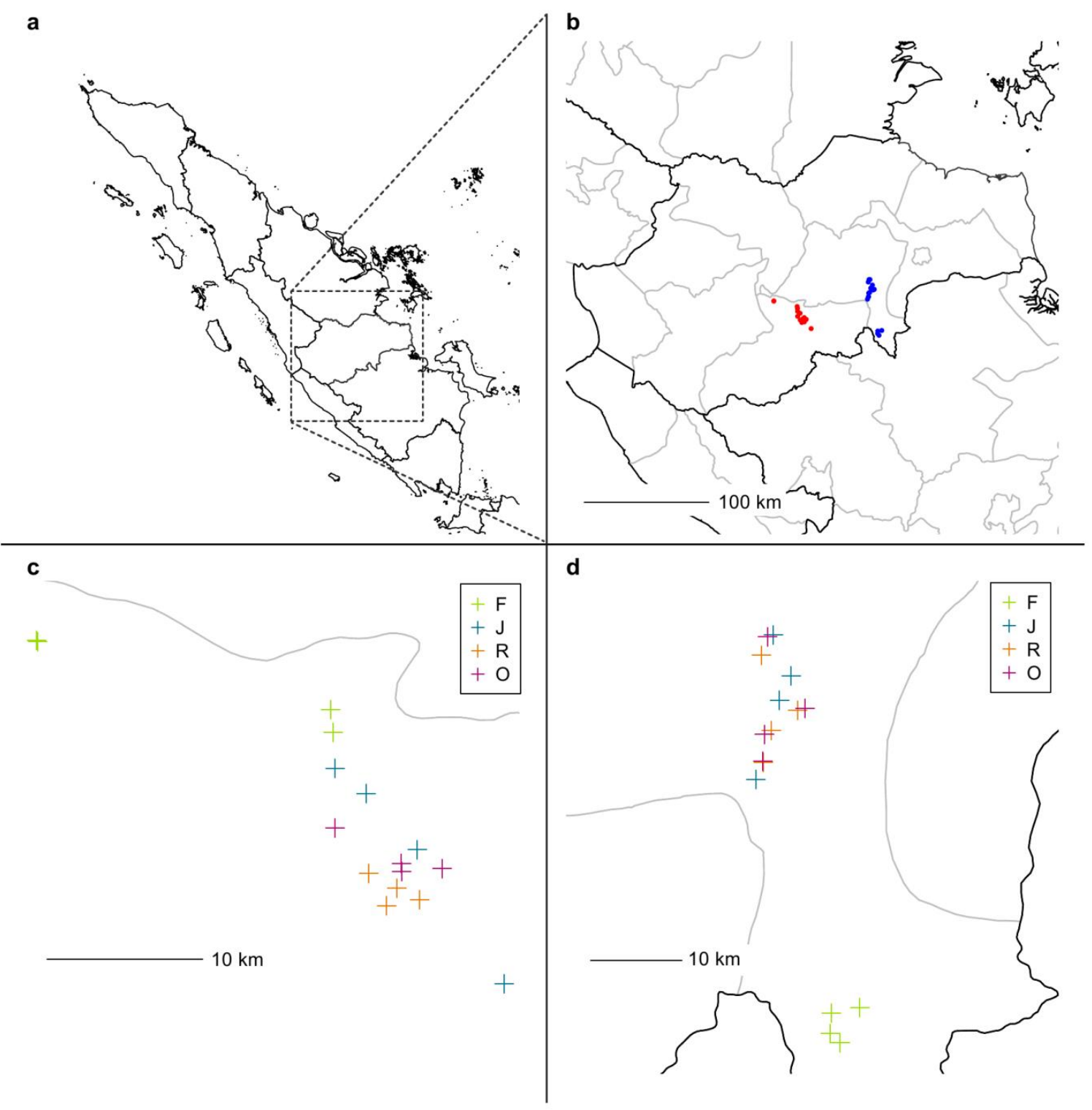


Supplementary Table 2.1 Full list of measured biotic variables organized into major taxonomic groups. Values are means of biomass and diversity (species richness) within each land-use system.

\begin{tabular}{|c|c|c|c|c|c|}
\hline Biotic variable & Unit & Forest & Jungle rubber & Rubber & Oil palm \\
\hline \multicolumn{6}{|l|}{ Plants } \\
\hline aboveground biomass & $\mathrm{Mg} \mathrm{ha}^{-1}$ & 321.42 & 115.28 & 61.06 & 42.30 \\
\hline belowground biomass & $\mathrm{Mg} \mathrm{ha}^{-1}$ & 70.28 & 37.46 & 21.49 & 18.92 \\
\hline diversity & & 274.50 & 158.75 & 61.63 & 71.13 \\
\hline \multicolumn{6}{|l|}{ Microbes } \\
\hline biomass & $\mathrm{mg} \mathrm{N} \mathrm{kg}^{-1}$ & 781.04 & 750.03 & 511.00 & 509.85 \\
\hline archaeal diversity & & 123.81 & 112.95 & 105.84 & 114.56 \\
\hline bacterial diversity & & 957.10 & 1052.08 & 1258.71 & 1373.77 \\
\hline \multicolumn{6}{|l|}{ Litter invertebrates } \\
\hline detritivore biomass & $\mathrm{mg} 3 \mathrm{~m}^{-2}$ & 103.89 & 55.79 & 50.39 & 35.24 \\
\hline detritivore diversity & & 22.50 & 23.63 & 17.63 & 13.38 \\
\hline herbivore biomass & $\operatorname{mg~} 3 \mathrm{~m}^{-2}$ & 9.29 & 13.94 & 31.90 & 7.63 \\
\hline herbivore diversity & & 3.88 & 5.75 & 7.88 & 4.38 \\
\hline omnivore biomass & $\operatorname{mg~} 3 \mathrm{~m}^{-2}$ & 62.93 & 49.45 & 75.08 & 76.65 \\
\hline omnivore diversity & & 26.63 & 18.75 & 18.88 & 14.50 \\
\hline predator biomass & $\mathrm{mg} 3 \mathrm{~m}^{-2}$ & 166.37 & 97.61 & 95.41 & 42.40 \\
\hline predator diversity & & 41.25 & 33.63 & 29.88 & 19.38 \\
\hline \multicolumn{6}{|l|}{ Arboreal ants } \\
\hline biomass & $\mathrm{mg} /$ bate station $\mathrm{hr}^{-1}$ & 8.89 & 10.65 & 10.61 & 32.07 \\
\hline diversity & & 9.88 & 8.11 & 13.38 & 13.33 \\
\hline \multicolumn{6}{|l|}{ Birds } \\
\hline herbivore biomass & g per plot & 2930.28 & 517.39 & 166.18 & 461.91 \\
\hline herbivore diversity & & 2.75 & 2.88 & 2.50 & 4.00 \\
\hline omnivore biomass & g per plot & 136.33 & 25.01 & 41.64 & 33.25 \\
\hline omnivore diversity & & 1.25 & 0.5 & 0.5 & 0.25 \\
\hline predator biomass & g per plot & 453.78 & 255.87 & 196.60 & 162.39 \\
\hline predator diversity & & 4.75 & 3.38 & 2.38 & 2.00 \\
\hline
\end{tabular}


Supplementary Figure 2.2 Directed acyclic diagram exhibiting all possible independence claims that were tested within the multilevel path model. Arrows leading from and to whole variable groups (variables enclosed within grey polygons) indicate that all variables within these groups are either respective predictor or response variables in such indicated models (for visual simplification). Black circular nodes between land-use change and the environmental variables indicate where interaction terms were also included in the independence claims.

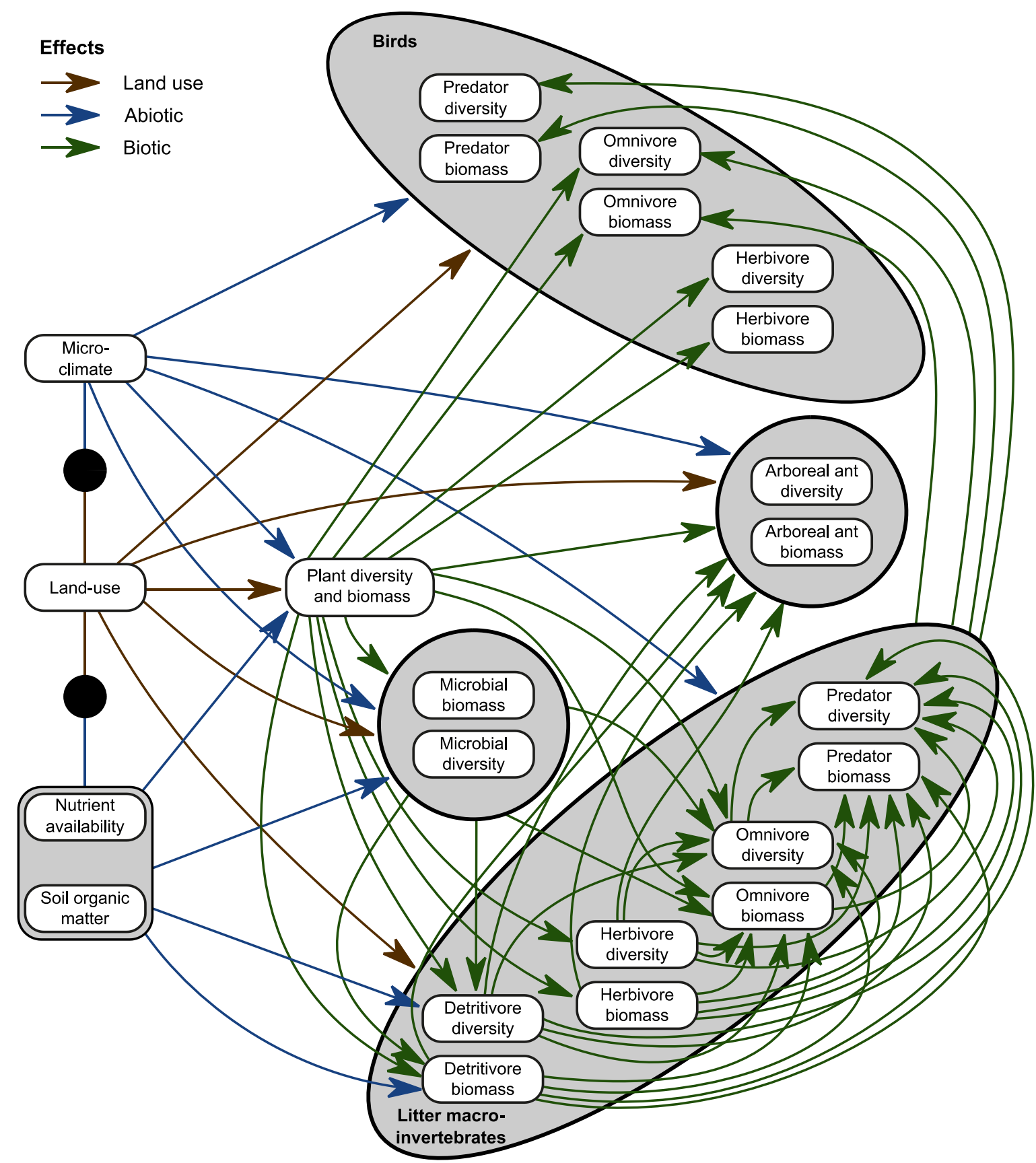


Supplementary Table 2.2 Model parameters for all dependence claims in the multilevel path model. Unstandardised coefficients and their respective standard errors are taken from the initial model with mean-centred variables. The $z$-scores column gives coefficients from the path model after being re-run with standardized values (mean-centred variables divided by twice their stand deviation). Marginal $R^{2}$ values indicate total proportion of variance explained by significant predictors and their conditioning variables in the significant models.

\begin{tabular}{|c|c|c|c|c|c|c|}
\hline $\begin{array}{l}\text { response } \\
\text { variable }\end{array}$ & fixed effects & $\begin{array}{l}\text { unstandardized } \\
\text { coefficients }\end{array}$ & std. error & $z$-score & p-value & $\underset{\mathbf{R}^{2}}{\operatorname{marginal}}$ \\
\hline \multirow[t]{2}{*}{ plant Div.Mass. } & land use & -4.090 & 0.180 & -1.194 & $<0.001$ & \multirow[t]{2}{*}{0.955} \\
\hline & land use*Nut.Ret. & -0.315 & 0.095 & -0.305 & 0.028 & \\
\hline \multirow[t]{3}{*}{ microbial Mass. } & land use & -0.199 & 0.081 & -0.563 & 0.002 & \multirow[t]{3}{*}{0.477} \\
\hline & Nut. Ret. & -0.053 & 0.014 & -0.496 & $<0.001$ & \\
\hline & $\begin{array}{l}\text { land use*soil } \\
\text { Org.Mat. }\end{array}$ & 0.084 & 0.055 & 0.669 & 0.019 & \\
\hline \multirow[t]{2}{*}{ microbial Div. } & land use & 662.743 & 136.534 & 1.483 & $<0.001$ & \multirow[t]{2}{*}{0.538} \\
\hline & microclimatic Var. & -86.477 & 36.765 & -0.572 & 0.027 & \\
\hline Arb.Ant Mass. & land use & 0.635 & 0.113 & 0.952 & $<0.001$ & 0.535 \\
\hline Arb.Ant Div. & land use & 3.546 & 1.580 & 0.445 & 0.004 & 0.305 \\
\hline Inv.Herb.Mass. & plant Div.Mass. & -0.727 & 0.327 & -2.068 & 0.038 & 0.001 \\
\hline \multirow[t]{2}{*}{ Inv.Herb.Div. } & land use & -1.501 & 0.531 & -2.568 & 0.019 & \multirow[t]{2}{*}{0.340} \\
\hline & plant Div.Mass. & -0.308 & 0.141 & -1.806 & 0.041 & \\
\hline Inv.Detr.Mass. & soil Org.Mat. & 0.220 & 0.061 & 0.559 & 0.001 & 0.302 \\
\hline \multirow[t]{3}{*}{ Inv.Detr.Div. } & land use & -1.169 & 0.283 & -3.149 & $<0.001$ & \multirow[t]{3}{*}{0.566} \\
\hline & plant Div.Mass. & -0.208 & 0.086 & -1.921 & 0.026 & \\
\hline & microbial Div. & 0.0005 & 0.0001 & 0.598 & 0.016 & \\
\hline \multirow[t]{4}{*}{ Inv.Omni.Mass. } & land use & -0.445 & 0.235 & -0.511 & $<0.001$ & \multirow[t]{4}{*}{0.784} \\
\hline & microclimatic Var. & -0.155 & 0.066 & -0.524 & 0.033 & \\
\hline & land use*Nut.Ret. & 0.328 & 0.084 & 1.250 & 0.003 & \\
\hline & $\begin{array}{l}\text { land use*soil } \\
\text { Org.Mat. }\end{array}$ & 0.740 & 0.170 & 2.384 & 0.002 & \\
\hline \multirow[t]{3}{*}{ Inv.Omni.Div. } & land use & -0.213 & 0.085 & -0.601 & 0.073 & \multirow[t]{3}{*}{0.722} \\
\hline & Inv.Herb.Div. & 0.293 & 0.082 & 0.827 & 0.002 & \\
\hline & $\begin{array}{l}\text { land use*soil } \\
\text { Org.Mat. }\end{array}$ & -0.125 & 0.064 & -0.351 & 0.009 & \\
\hline Inv.Pred.Mass. & land use & -0.706 & 0.167 & -0.860 & 0.002 & 0.383 \\
\hline \multirow[t]{2}{*}{ Inv.Pred.Div. } & Inv.Detr.Div. & 0.757 & 0.180 & 0.572 & $<0.001$ & \multirow[t]{2}{*}{0.702} \\
\hline & Inv.Omni.Div. & 0.713 & 0.256 & 0.514 & 0.012 & \\
\hline \multirow[t]{3}{*}{ bird Pred.Mass. } & microclimatic Var. & -129.419 & 33.159 & -0.755 & $<0.001$ & \multirow[t]{3}{*}{0.349} \\
\hline & arboreal ant Mass. & 538.736 & 190.126 & 0.709 & 0.010 & \\
\hline & Inv.Herb.Div. & 334.541 & 160.412 & 0.386 & 0.049 & \\
\hline
\end{tabular}




$\begin{array}{llrrrrr}\text { bird Pred. Div. } & \text { microclimatic Var. } & -0.075 & 0.025 & -0.484 & 0.007 & 0.489 \\ & \text { Inv.Pred.Mass. } & 0.306 & 0.102 & 0.507 & 0.009 & \\ \text { Inv.Pred.Div. } & -0.701 & 0.261 & -0.747 & 0.013 & \\ \text { Inv.Herb.Div. } & 0.407 & 0.124 & 0.516 & 0.004 & \end{array}$


Supplementary Figure 2.3 A) Series of principal component analysis (PCA) plots of each of the composited variables (row 1: soil physical component, and combined soil physical and biochemical component; row 2 : microclimatic component, and plant diversity and biomass component) used in the multilevel path model. The eight replicate plots of the four land-use types are plotted along PCA axes 1 and 2 (proportion of explained variance in parentheses) with red vectors (parameters included in PCA composite) illustrating factor loadings. Land-use systems are forest $(\mathrm{F})$, jungle rubber $(\mathrm{J})$, rubber monoculture $(\mathrm{R})$, and oil palm monoculture $(\mathrm{O})$. B) Relationships among the PCA scores for each composited variable and the parameters incorporated within the composited variables, which have PCA loadings of $>0.400$. Data points represent the eight replicate plots of the four land-use types.
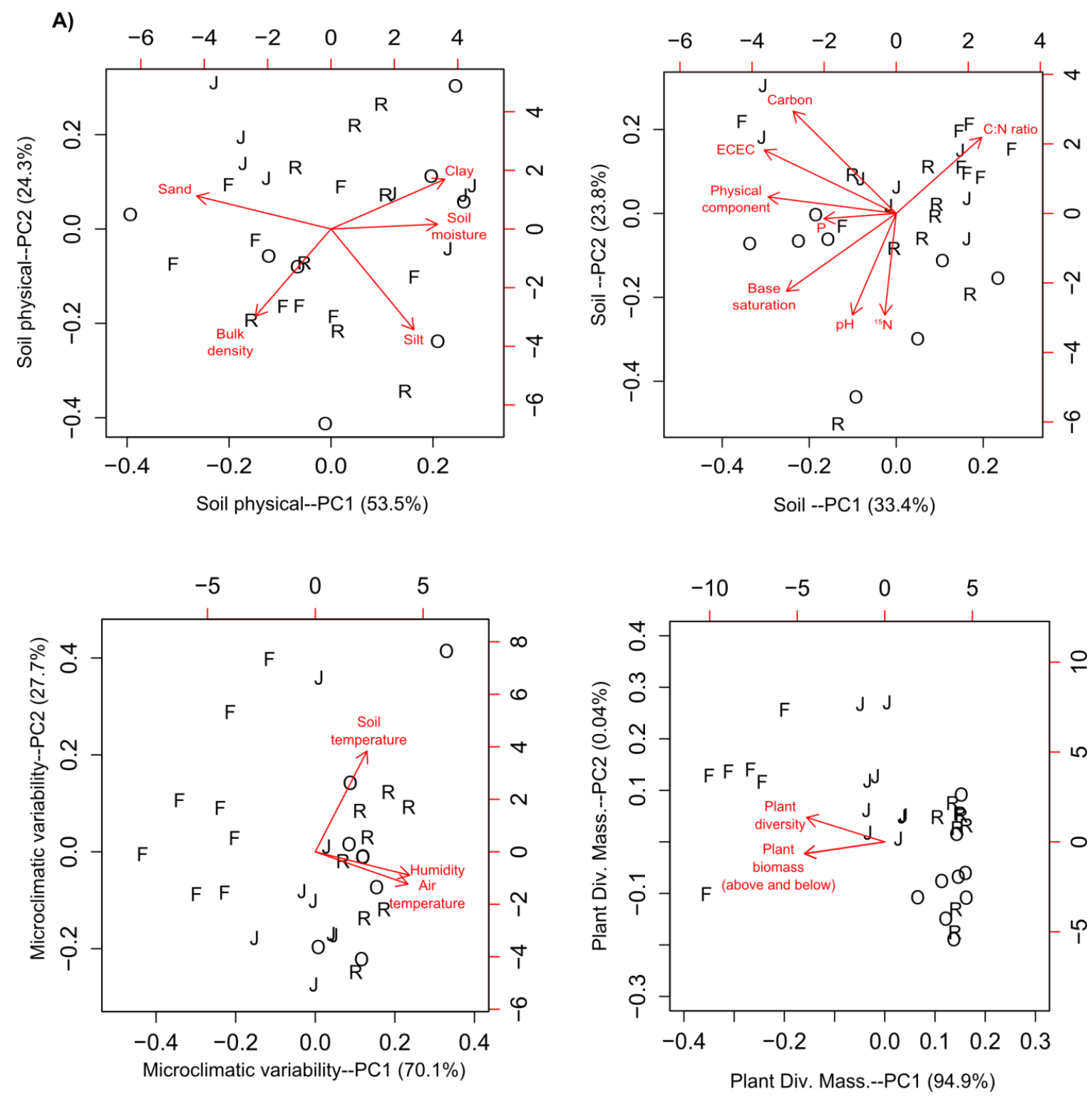
B)

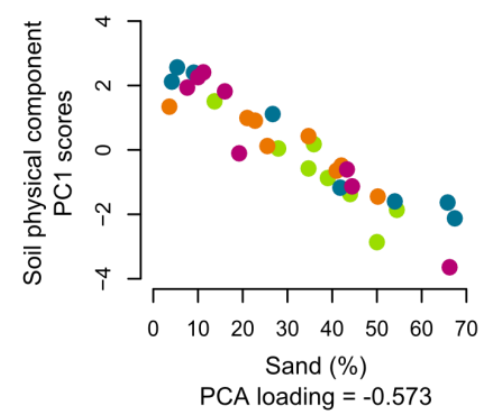

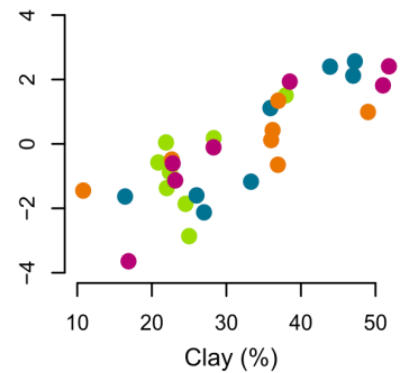

PCA loading $=0.485$
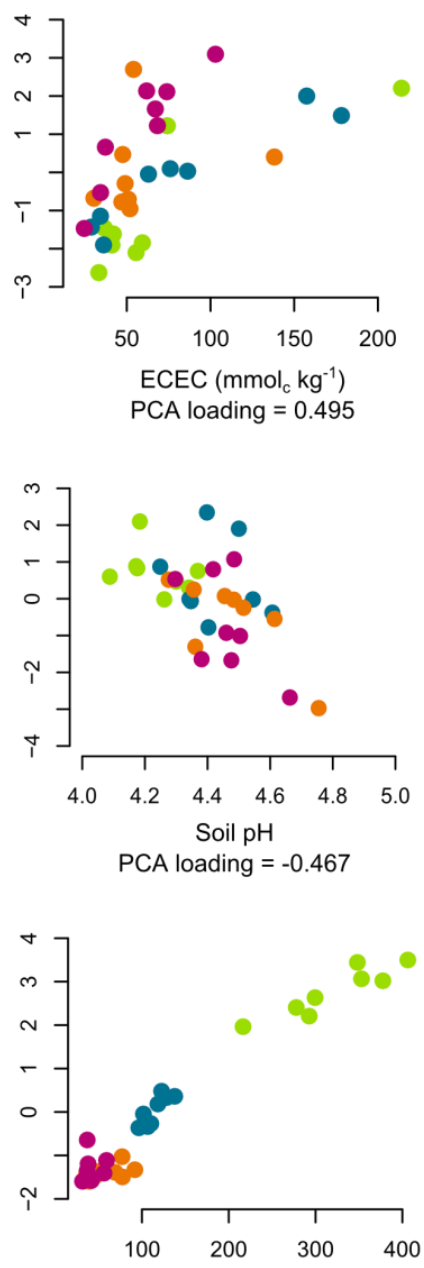

Above-ground biomass ( $\left.\mathrm{Mg} \mathrm{ha}^{-1}\right)$ PCA loading $=0.583$

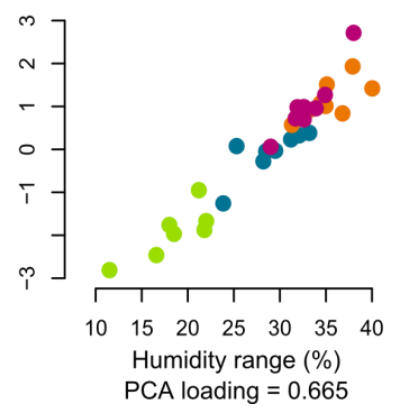

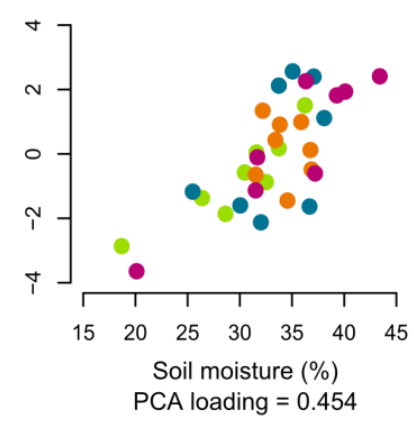
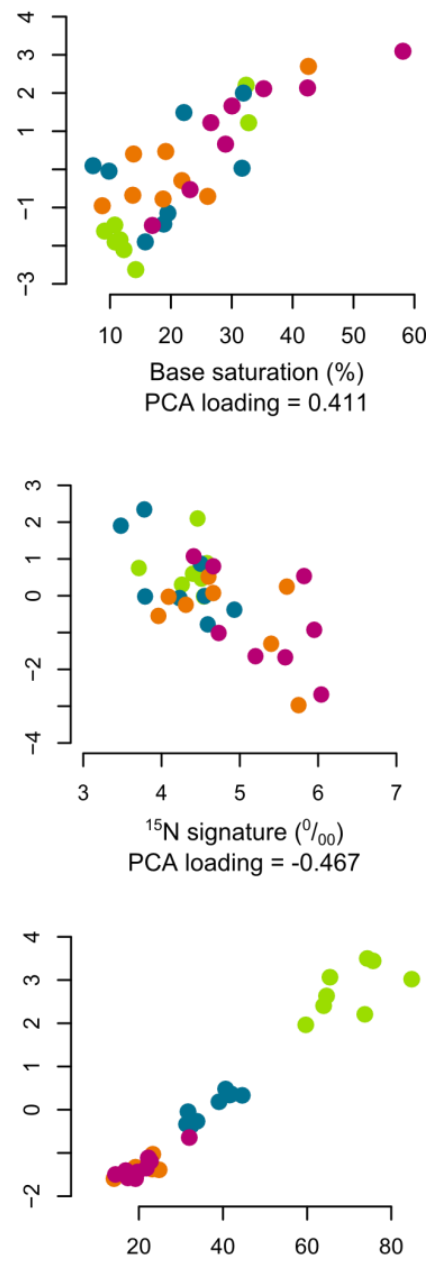

Below-ground biomass ( $\mathrm{Mg} \mathrm{ha}^{-1}$ ) $\mathrm{PCA}$ loading $=0.582$
- Forest
- Jungle rubber
- Rubber
- Oil palm 
Supplementary Figure 2.4 Box plots of all biotic variables across the four land-use systems that responded significantly to land-use change within the multilevel path model. Land-use systems are forest $(F)$, jungle rubber $(J)$, rubber monoculture $(R)$, and oil palm monoculture $(O)$.

Plant diversity \& biomass

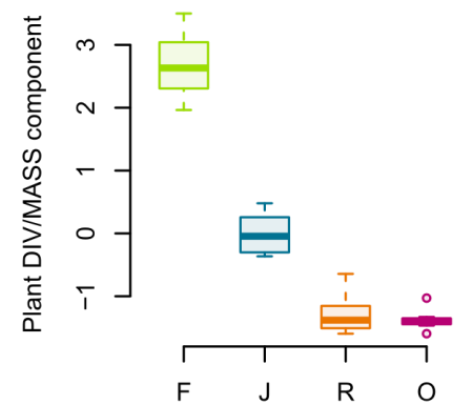

Litter invertebrates

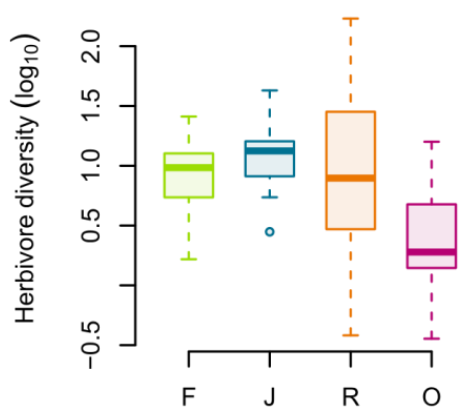

Litter invertebrates

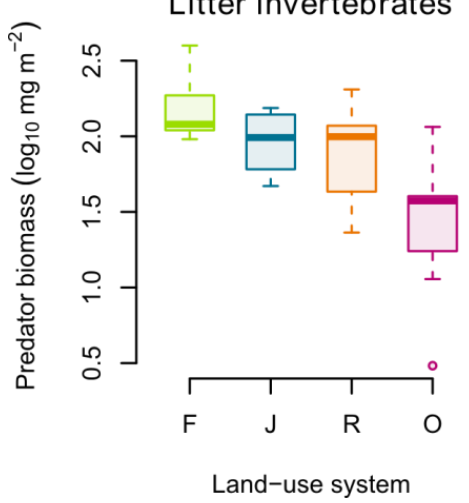

Soil microbes

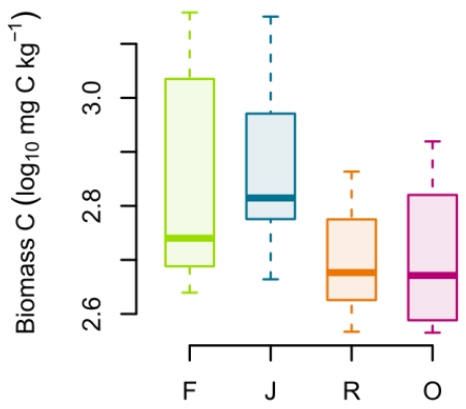

Litter invertebrates

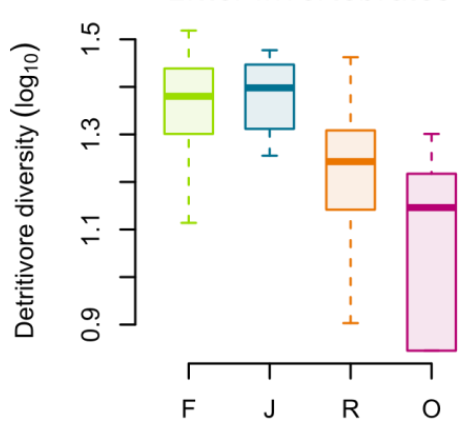

Arboreal ants

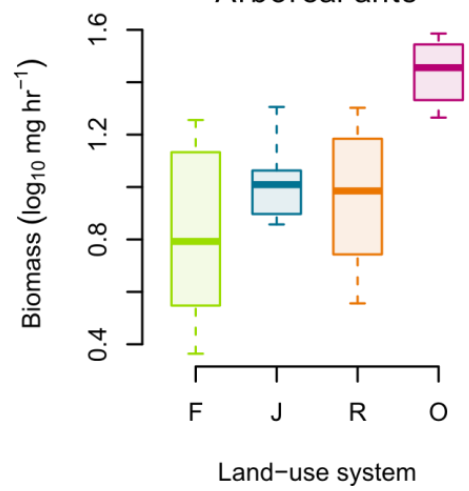

Soil microbes

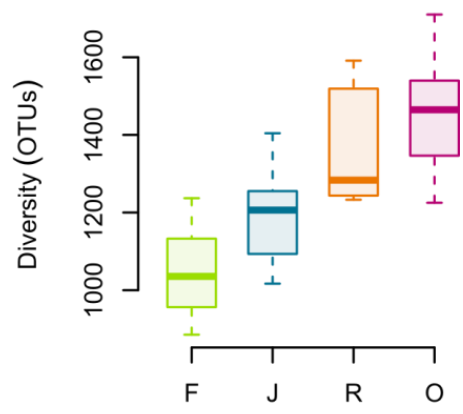

Litter invertebrates

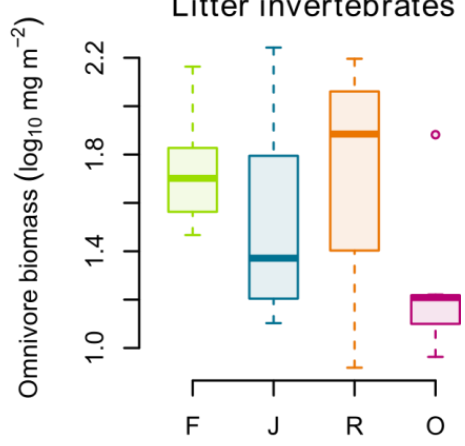

Arboreal ants

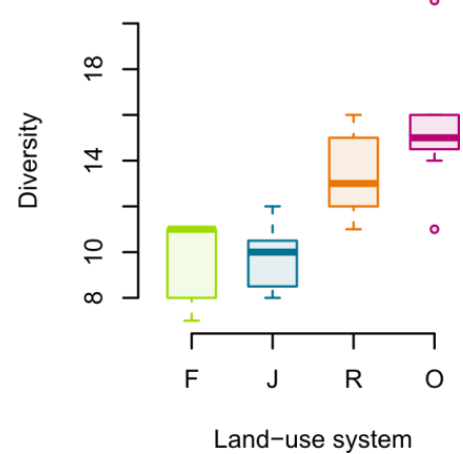


Supplementary Figure 2.5 Pairwise relationships among all response and predictor variables where significant direct effects were detected within the multilevel path model. Points are coloured according to land-use system, with their respective coloured model fits plotted where land-use change was found to have a significant effect in the multilevel path model. Model fits for the overall pairwise relationships (tested in isolation, excluding all conditioning variables that were included in the complete path model) are shown by a black line, with their respective pvalues indicated on each panel. 'DIV' and 'MASS' stand for diversity and biomass, respectively.
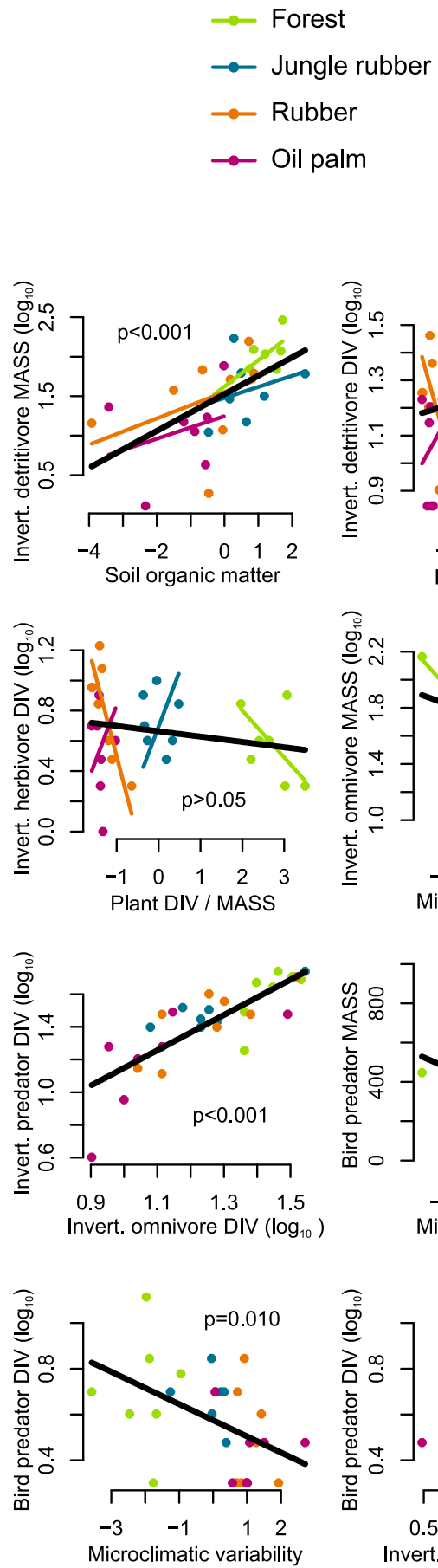
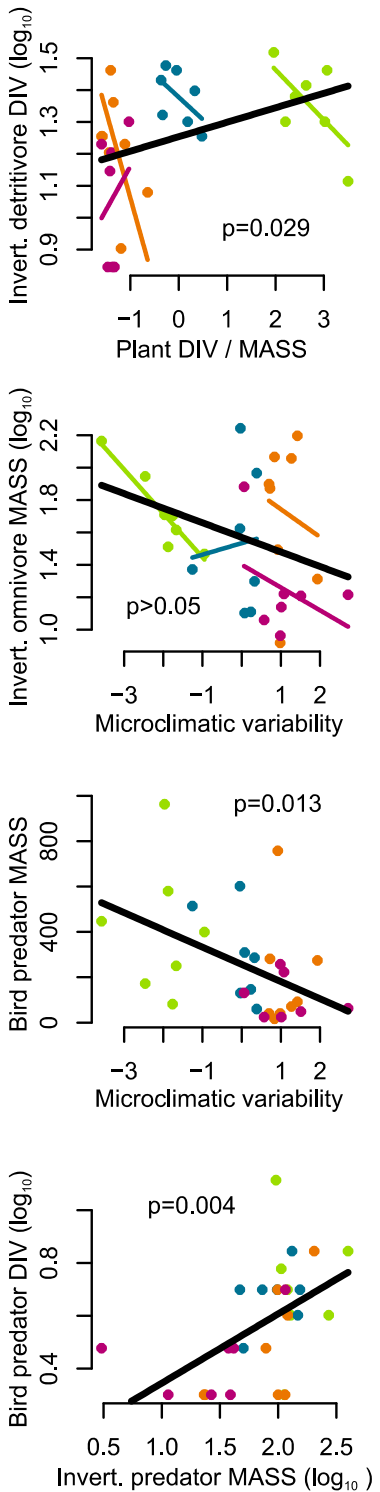
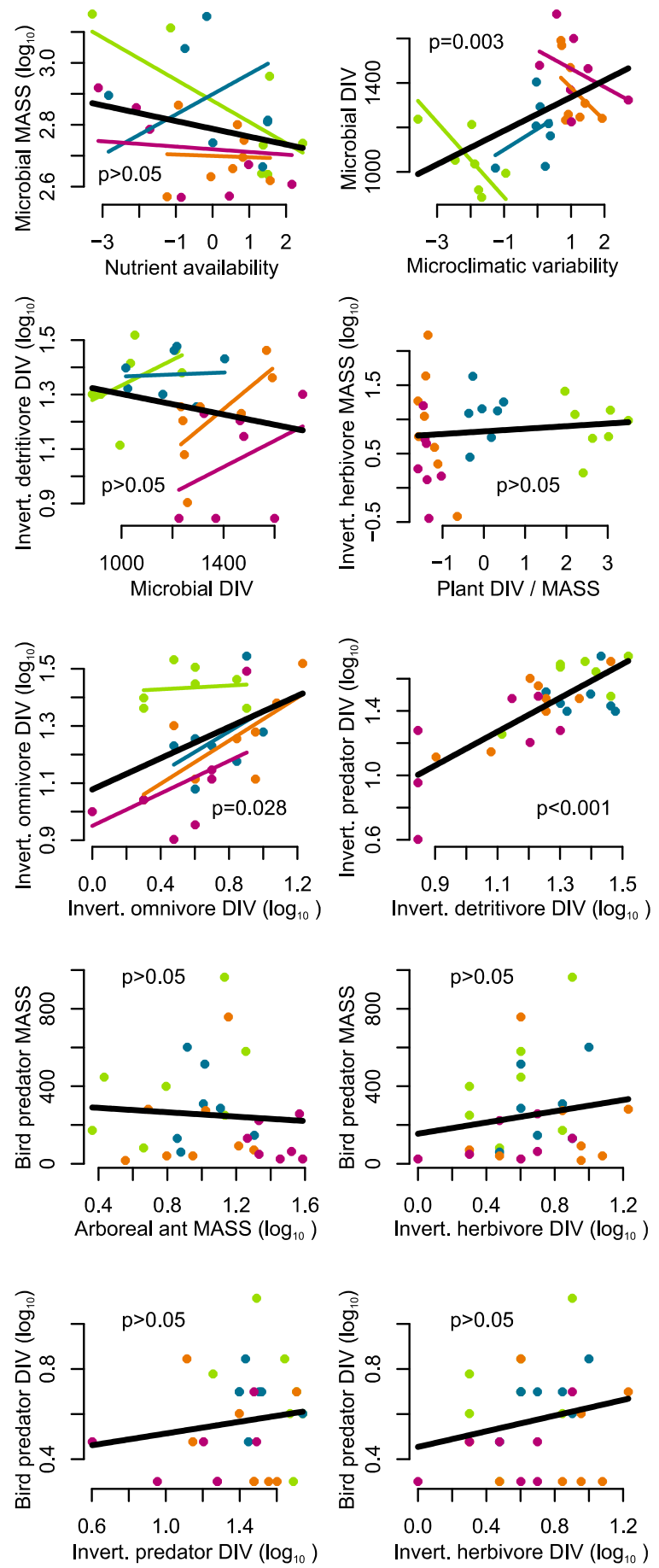


\section{SUPPLEMENTARY INFORMATION - CHAPTER 3}

Supplementary Figure 3.1 Map of the study region with an overview of Sumatra (a) and Jambi Province (b) with red and blue points denoting the 16 sites in Bukit Duabelas landscape and the 16 sites in Harapan landscape, respectively. Additionally, the spatial layout of the sampling sites in Bukit Duabelas landscape (c) and Harapan landscape (d) is represented by coloured crosses for forest $(F)$, jungle rubber $(J)$, rubber $(R)$ and oil palm $(\mathrm{O})$.

a

$\underbrace{\mathbf{b}}$

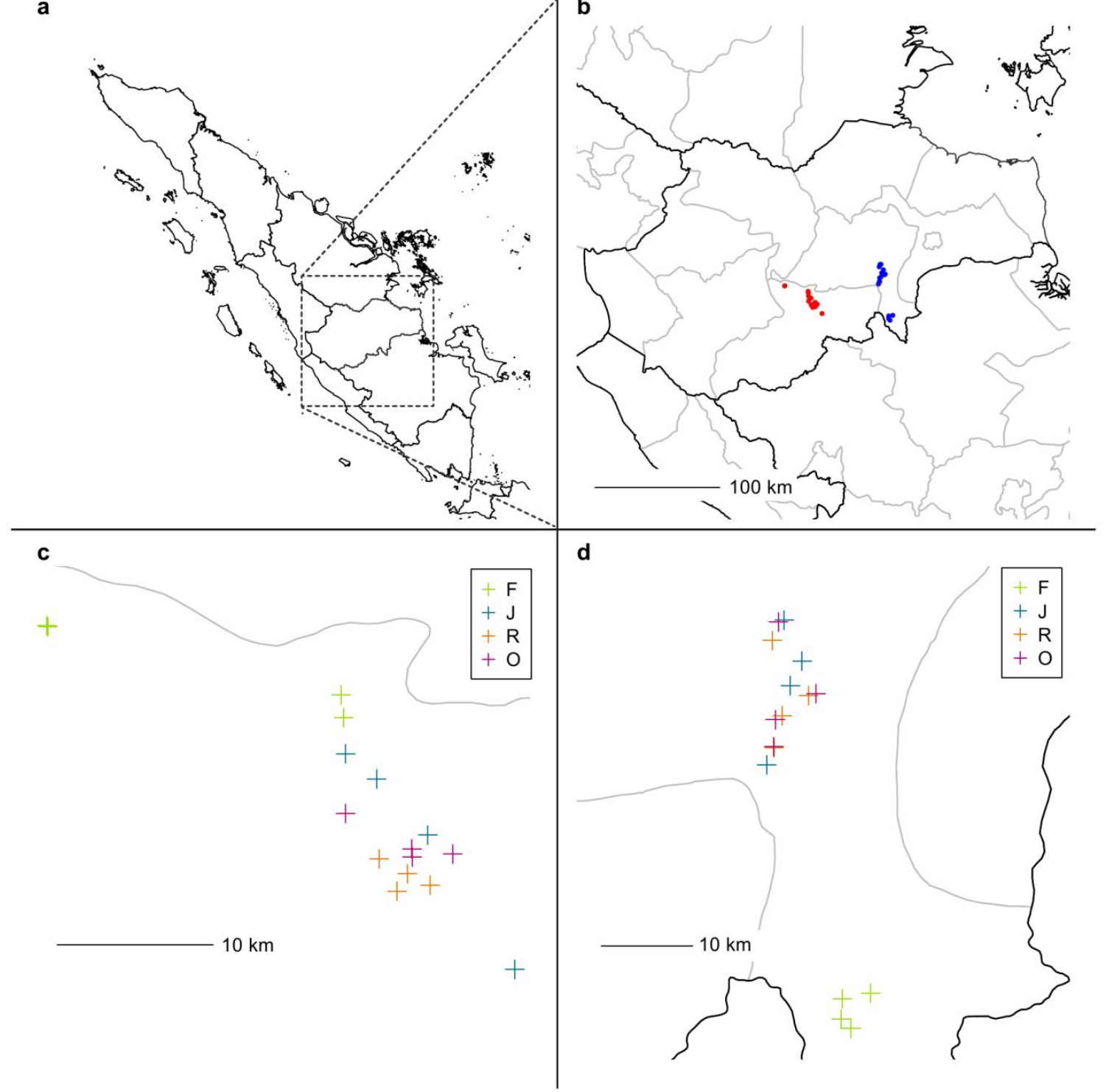


Supplementary Figure 3.2 Mean ( \pm SE) observed species richness (a), 2nd order jacknife extrapolated species richness (b) and rarefied species richness (c) for the four land-use transformation systems: forest $(\mathrm{F})$, jungle rubber $(\mathrm{J})$, rubber $(\mathrm{R})$ and oil palm $(\mathrm{O})$. $\rho$-values denote Pearson correlation coefficients between observed species richness and extrapolated (b) and rarefied species richness (c) for the 32 sites $(n=32)$, respectively.
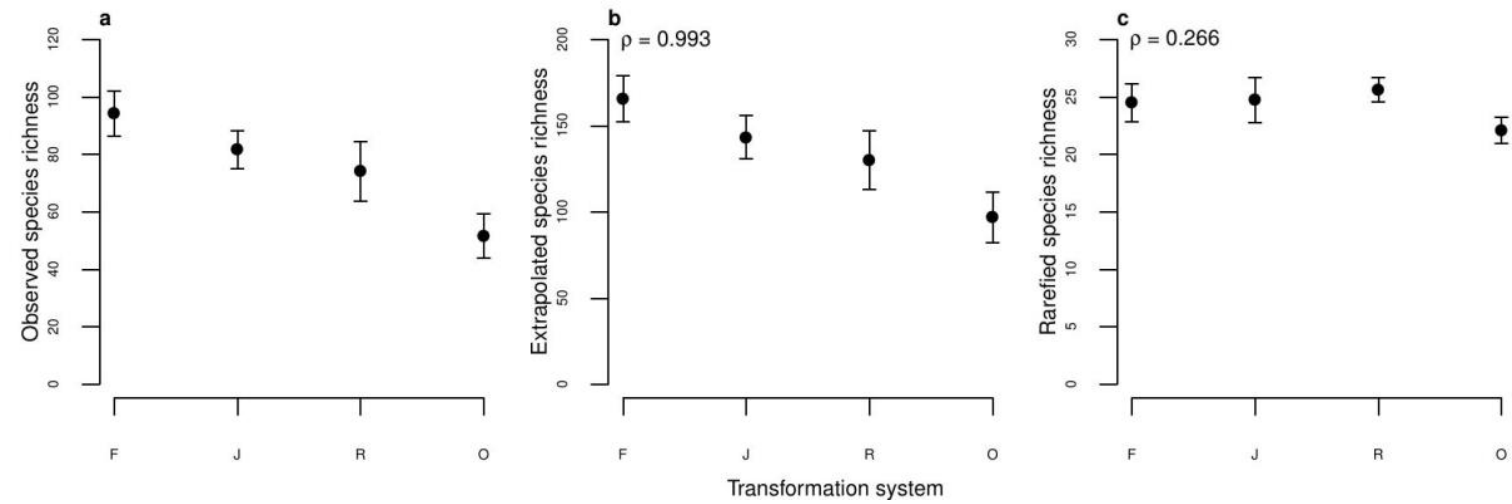

Supplementary Figure 3.3 (a) Comparative mean percentage change in total energy flux for the four transformation systems between our feeding link assumption null-model (Supplementary Methods) and an alternative flux calculation with omnivores consuming only live plant material and detritus (50\% each). Error bars denote upper and lower limits of absolute deviation from the mean. (b) Mean percentage loss in energy flux of the three agriculturally used transformation systems compared to the forest system. Dark grey and light grey bars denote the null model and alternative model calculations, respectively. Transformation system abbreviations are: forest (F), jungle rubber $(\mathrm{J})$, rubber $(\mathrm{R})$ and oil palm $(\mathrm{O})$.
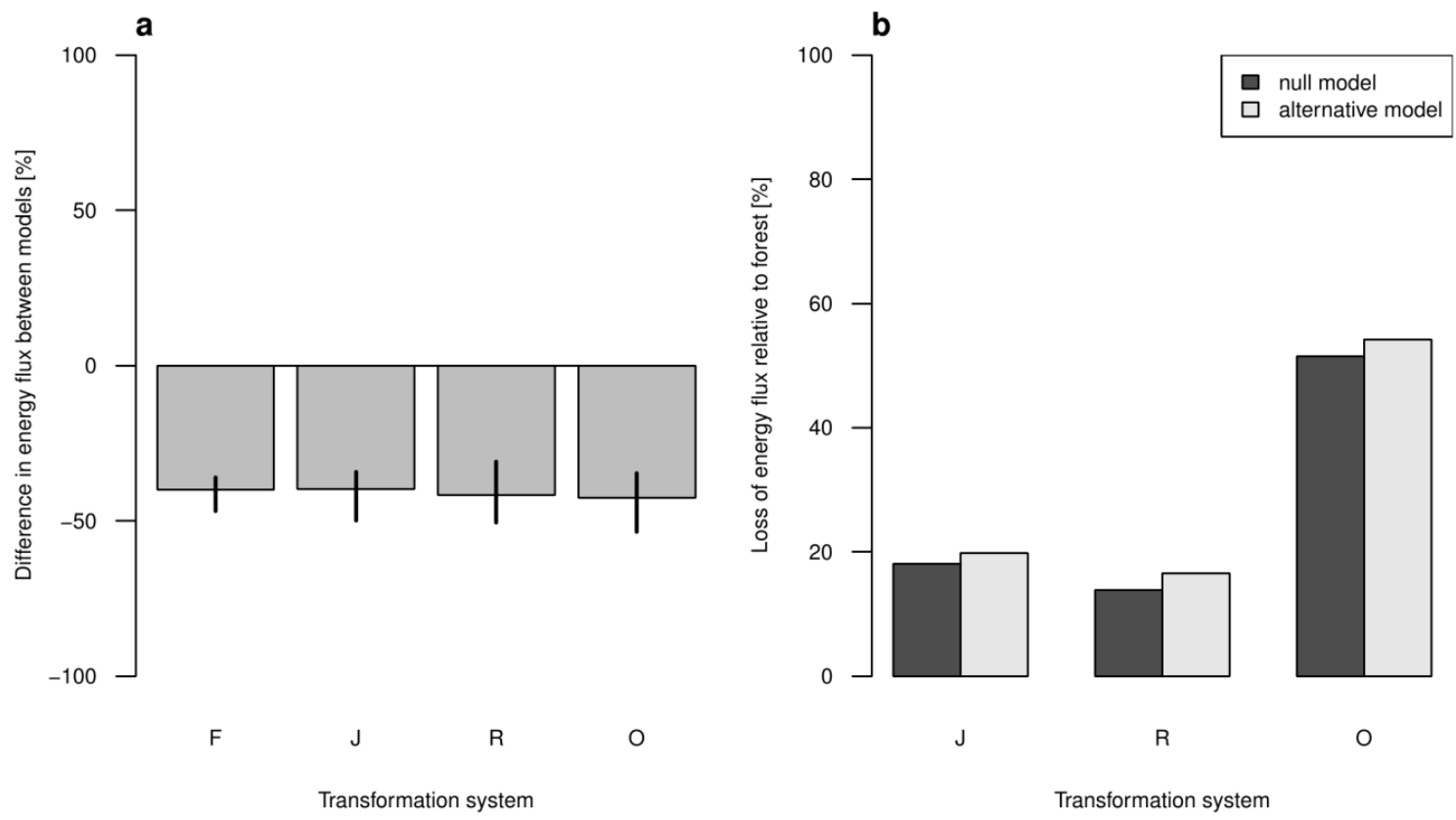
Supplementary Figure 3.4 Body mass distributions across the four transformation systems for each of the four functional feeding guilds: omnivores (3209 individuals), detritivores (2242 individuals), predators (1766 individuals), and herbivores (255 individuals).
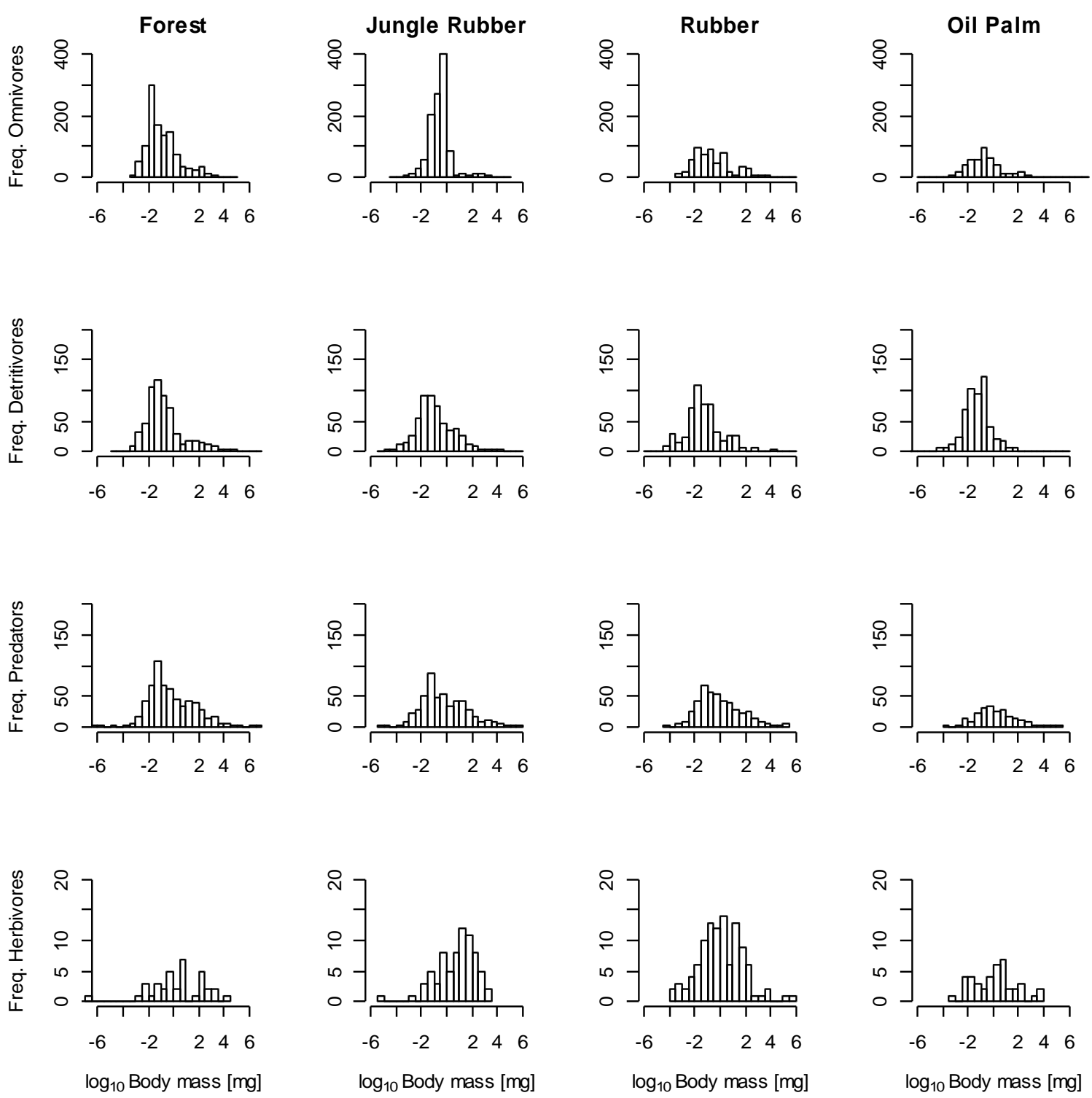
Supplementary Table 3.1a Length-mass regression parameters for calculation of individual body masses from measured body lengths. For damaged individuals where body length could not be measured ( 66 of 7472 individuals), body mass was substituted by species median body mass or order median body mass (for species with single individuals). 'Taxon', 'Group' and 'Further grouping' specify which animals the presented regression has been used for in this study. Regressions were available from the literature that estimate both dry and fresh mass ('Mass type') for different taxa. Supplementary table $4 \mathrm{~b}$ presents the dry mass-fresh mass conversions, used to convert all estimated body masses to fresh mass. The equations and regression parameters, 'a' and 'b', are presented, as well as the size range the regressions were calculated from ('Min' and 'Max'). All regressions were taken from the literature ('Reference'), with different specific definitions of how body length was measured ('Details of body length measurement') and specificity of the given regression ('Regression specificity').

\begin{tabular}{|c|c|c|c|c|c|c|c|c|c|c|c|}
\hline Taxon & Group & $\begin{array}{l}\text { Further } \\
\text { grouping }\end{array}$ & Mass type & Equation $\mathrm{M}[\mathrm{mg}], \mathrm{L}[\mathrm{mm}]$ & a & b & $\operatorname{Min}(\mathbf{m m})$ & $\operatorname{Max}(\mathrm{mm})$ & Reference & Details of body length measurement & $\begin{array}{l}\text { Regression } \\
\text { specificity }\end{array}$ \\
\hline Annelida & All & & $\begin{array}{l}\text { ash free dry } \\
\text { mass }\end{array}$ & $\mathrm{M}=1000 * \exp (\mathrm{a}+\mathrm{b} * \log (\mathrm{L}))$ & -11.8423 & 2.3225 & & & (Hale, Reich \& Frelich, 2004) & Total length & $\begin{array}{l}\text { General } \\
\text { Lumbricidae }\end{array}$ \\
\hline Araneae & Araneae $<2.5 \mathrm{~mm}$ & & Fresh mass & $\mathrm{M}=\exp (\mathrm{a}+\mathrm{b} * \log (\mathrm{L}))$ & -1.958 & 2.746 & 0.56 & 2.5 & (Höfer \& Ott, 2009) & $\begin{array}{l}\text { edge of prosoma (without chelicerae) to edge of } \\
\text { opisthosoma (excl spinnerets) }\end{array}$ & Group specific \\
\hline Araneae & hunting & & Fresh mass & $M=\exp (a+b * \log (L))$ & -2.108 & 3.017 & 0.67 & 36 & (Höfer \& Ott, 2009) & $\begin{array}{l}\text { edge of prosoma (without chelicerae) to edge of } \\
\text { opisthosoma (excl spinnerets) }\end{array}$ & Group specific \\
\hline Araneae & web-building & & Fresh mass & $\mathrm{M}=\exp (\mathrm{a}+\mathrm{b} * \log (\mathrm{L}))$ & -1.784 & 2.255 & 0.56 & 10.67 & (Höfer \& Ott, 2009) & $\begin{array}{l}\text { edge of prosoma (without chelicerae) to edge of } \\
\text { opisthosoma (excl spinnerets) }\end{array}$ & Group specific \\
\hline Araneae & spiders random & & fresh mass & $M=\exp (a+b * \log (L))$ & -1.844 & 2.711 & 1.8 & 21.5 & (Edwards, 1996) & clypeus to tip of spinnerets & Group specific \\
\hline Araneae & Anapidae & & Fresh mass & $\mathrm{M}=\exp (\mathrm{a}+\mathrm{b} * \log (\mathrm{L}))$ & -1.784 & 2.255 & 0.56 & 10.67 & (Höfer \& Ott, 2009) & $\begin{array}{l}\text { edge of prosoma (without chelicerae) to edge of } \\
\text { opisthosoma (excl spinnerets) }\end{array}$ & $\begin{array}{l}\text { inferred, web- } \\
\text { building }\end{array}$ \\
\hline Araneae & Araneidae & & Fresh mass & $M=\exp (a+b * \log (L))$ & -1.923 & 2.923 & 2.10 & 21.20 & (Edwards, 1996) & clypeus to tip of spinnerets & Group specific \\
\hline Araneae & Barychelidae & & Fresh mass & $\mathrm{M}=\exp (\mathrm{a}+\mathrm{b} * \log (\mathrm{L}))$ & -2.108 & 3.017 & 0.67 & 36 & (Höfer \& Ott, 2009) & $\begin{array}{l}\text { edge of prosoma (without chelicerae) to edge of } \\
\text { opisthosoma (excl spinnerets) }\end{array}$ & $\begin{array}{l}\text { inferred, hunting } \\
\text { spiders }\end{array}$ \\
\hline Araneae & Clubionidae & & Fresh mass & $M=\exp (a+b * \log (L))$ & -2.156 & 2.653 & 2.5 & 9 & (Edwards, 1996) & clypeus to tip of spinnerets & Group specific \\
\hline Araneae & Corinnidae & & Fresh mass & $M=\exp (a+b * \log (L))$ & -2.108 & 3.017 & 0.67 & 36 & (Höfer \& Ott, 2009) & $\begin{array}{l}\text { edge of prosoma (without chelicerae) to edge of } \\
\text { opisthosoma (excl spinnerets) }\end{array}$ & $\begin{array}{l}\text { inferred, hunting } \\
\text { spiders }\end{array}$ \\
\hline Araneae & Ctenidae & & Fresh mass & $\mathrm{M}=\exp (\mathrm{a}+\mathrm{b} * \log (\mathrm{L}))$ & -1.758 & 2.894 & 1.3 & 36 & (Höfer \& Ott, 2009) & $\begin{array}{l}\text { edge of prosoma (without chelicerae) to edge of } \\
\text { opisthosoma (excl spinnerets) }\end{array}$ & Group specific \\
\hline Araneae & Deinopidae & & fresh mass & $\mathrm{M}=\exp (\mathrm{a}+\mathrm{b} * \log (\mathrm{L}))$ & -1.844 & 2.711 & 1.8 & 21.5 & (Edwards, 1996) & clypeus to tip of spinnerets & $\begin{array}{l}\text { inferred, spiders } \\
\text { random sample }\end{array}$ \\
\hline Araneae & Gnaphosidae & & Fresh mass & $\mathrm{M}=\exp (\mathrm{a}+\mathrm{b} * \log (\mathrm{L}))$ & -2.83 & 3.055 & 3 & 13.1 & (Edwards, 1996) & clypeus to tip of spinnerets & Group specific \\
\hline Araneae & Hexathelidae & & Fresh mass & $M=\exp (a+b * \log (L))$ & -2.108 & 3.017 & 0.67 & 36 & (Höfer \& Ott, 2009) & $\begin{array}{l}\text { edge of prosoma (without chelicerae) to edge of } \\
\text { opisthosoma (excl spinnerets) }\end{array}$ & $\begin{array}{l}\text { inferred, hunting } \\
\text { spiders }\end{array}$ \\
\hline
\end{tabular}




\begin{tabular}{|c|c|c|c|c|c|c|c|c|c|c|}
\hline Araneae & Lamponidae & Fresh mass & $\mathrm{M}=\exp (\mathrm{a}+\mathrm{b} * \log (\mathrm{L}))$ & -2.83 & 3.055 & 3 & 13.1 & (Edwards, 1996) & clypeus to tip of spinnerets & $\begin{array}{l}\text { inferred, } \\
\text { Gnaphosidae }\end{array}$ \\
\hline Araneae & Linyphiidae & Fresh mass & $\mathrm{M}=\exp (\mathrm{a}+\mathrm{b} * \log (\mathrm{L}))$ & -1.892 & 2.754 & 1.5 & 5.5 & (Edwards, 1996) & clypeus to tip of spinnerets & Group specific \\
\hline Araneae & Lycosidae & Fresh mass & $M=\exp (a+b * \log (L))$ & -2.043 & 2.842 & 2 & 23.5 & (Edwards, 1996) & clypeus to tip of spinnerets & Group specific \\
\hline Araneae & Micropholcommatidae & Fresh mass & $\mathrm{M}=\exp (\mathrm{a}+\mathrm{b} * \log (\mathrm{L}))$ & -1.784 & 2.255 & 0.56 & 10.67 & (Höfer \& Ott, 2009) & $\begin{array}{l}\text { edge of prosoma (without chelicerae) to edge of } \\
\text { opisthosoma (excl spinnerets) }\end{array}$ & $\begin{array}{l}\text { inferred, web- } \\
\text { building }\end{array}$ \\
\hline Araneae & Miturgidae & Fresh mass & $\mathrm{M}=\exp (\mathrm{a}+\mathrm{b} * \log (\mathrm{L}))$ & -2.83 & 3.055 & 3 & 13.1 & (Edwards, 1996) & clypeus to tip of spinnerets & $\begin{array}{l}\text { inferred, } \\
\text { Gnaphosidae }\end{array}$ \\
\hline Araneae & Mysmenidae & Fresh mass & $M=\exp (a+b * \log (L))$ & -1.784 & 2.255 & 0.56 & 10.67 & (Höfer \& Ott, 2009) & $\begin{array}{l}\text { edge of prosoma (without chelicerae) to edge of } \\
\text { opisthosoma (excl spinnerets) }\end{array}$ & $\begin{array}{l}\text { inferred, web- } \\
\text { building }\end{array}$ \\
\hline Araneae & Nemesiidae & Fresh mass & $M=\exp (a+b * \log (L))$ & -2.108 & 3.017 & 0.67 & 36 & (Höfer \& Ott, 2009) & $\begin{array}{l}\text { edge of prosoma (without chelicerae) to edge of } \\
\text { opisthosoma (excl spinnerets) }\end{array}$ & $\begin{array}{l}\text { inferred, hunting } \\
\text { spiders }\end{array}$ \\
\hline Araneae & Nephilidae & Fresh mass & $M=\exp (a+b * \log (L))$ & -1.784 & 2.255 & 0.56 & 10.67 & (Höfer \& Ott, 2009) & $\begin{array}{l}\text { edge of prosoma (without chelicerae) to edge of } \\
\text { opisthosoma (excl spinnerets) }\end{array}$ & $\begin{array}{l}\text { inferred, web- } \\
\text { building }\end{array}$ \\
\hline Araneae & Ochyroceratidae & Fresh mass & $\mathrm{M}=\exp (\mathrm{a}+\mathrm{b} * \log (\mathrm{L}))$ & -1.784 & 2.255 & 0.56 & 10.67 & (Höfer \& Ott, 2009) & $\begin{array}{l}\text { edge of prosoma (without chelicerae) to edge of } \\
\text { opisthosoma (excl spinnerets) }\end{array}$ & $\begin{array}{l}\text { inferred, web- } \\
\text { building }\end{array}$ \\
\hline Araneae & Oonopidae & Fresh mass & $M=\exp (a+b * \log (L))$ & -2.039 & 2.666 & 0.67 & 2.5 & (Höfer \& Ott, 2009) & $\begin{array}{l}\text { edge of prosoma (without chelicerae) to edge of } \\
\text { opisthosoma (excl spinnerets) }\end{array}$ & Group specific \\
\hline Araneae & Oxyopidae & Fresh mass & $M=\exp (a+b * \log (L))$ & -2.108 & 3.017 & 0.67 & 36 & (Höfer \& Ott, 2009) & $\begin{array}{l}\text { edge of prosoma (without chelicerae) to edge of } \\
\text { opisthosoma (excl spinnerets) }\end{array}$ & $\begin{array}{l}\text { inferred, hunting } \\
\text { spiders }\end{array}$ \\
\hline Araneae & Palpimanidae & Fresh mass & $\mathrm{M}=\exp (\mathrm{a}+\mathrm{b} * \log (\mathrm{L}))$ & -2.108 & 3.017 & 0.67 & 36 & (Höfer \& Ott, 2009) & $\begin{array}{l}\text { edge of prosoma (without chelicerae) to edge of } \\
\text { opisthosoma (excl spinnerets) }\end{array}$ & $\begin{array}{l}\text { inferred, hunting } \\
\text { spiders }\end{array}$ \\
\hline Araneae & Pararchaeidae & Fresh mass & $M=\exp (a+b * \log (L))$ & -2.108 & 3.017 & 0.67 & 36 & (Höfer \& Ott, 2009) & $\begin{array}{l}\text { edge of prosoma (without chelicerae) to edge of } \\
\text { opisthosoma (excl spinnerets) }\end{array}$ & $\begin{array}{l}\text { inferred, hunting } \\
\text { spiders }\end{array}$ \\
\hline Araneae & Philodromidae & Fresh mass & $\mathrm{M}=\exp (\mathrm{a}+\mathrm{b} * \log (\mathrm{L}))$ & -1.985 & 2.940 & 2.50 & 8.60 & (Edwards, 1996) & clypeus to tip of spinnerets & Group specific \\
\hline Araneae & Pholcidae & Fresh mass & $\mathrm{M}=\exp (\mathrm{a}+\mathrm{b} * \log (\mathrm{L}))$ & -1.784 & 2.255 & 0.56 & 10.67 & (Höfer \& Ott, 2009) & $\begin{array}{l}\text { edge of prosoma (without chelicerae) to edge of } \\
\text { opisthosoma (excl spinnerets) }\end{array}$ & $\begin{array}{l}\text { inferred, web- } \\
\text { building }\end{array}$ \\
\hline Araneae & Prodidomidae & Fresh mass & $\mathrm{M}=\exp (\mathrm{a}+\mathrm{b} * \log (\mathrm{L}))$ & -2.83 & 3.055 & 3 & 13.1 & (Edwards, 1996) & clypeus to tip of spinnerets & $\begin{array}{l}\text { inferred, } \\
\text { Gnaphosidae } \\
\end{array}$ \\
\hline Araneae & Salticidae & Fresh mass & $\mathrm{M}=\exp (\mathrm{a}+\mathrm{b} * \log (\mathrm{L}))$ & -2.184 & 2.901 & 4.00 & 13.00 & (Edwards, 1996) & clypeus to tip of spinnerets & Group specific \\
\hline Araneae & Scytodidae & Fresh mass & $M=\exp (a+b * \log (L))$ & -2.108 & 3.017 & 0.67 & 36 & (Höfer \& Ott, 2009) & $\begin{array}{l}\text { edge of prosoma (without chelicerae) to edge of } \\
\text { opisthosoma (excl spinnerets) }\end{array}$ & $\begin{array}{l}\text { inferred, hunting } \\
\text { spiders }\end{array}$ \\
\hline Araneae & Segestriidae & Fresh mass & $M=\exp (a+b * \log (L))$ & -2.108 & 3.017 & 0.67 & 36 & (Höfer \& Ott, 2009) & $\begin{array}{l}\text { edge of prosoma (without chelicerae) to edge of } \\
\text { opisthosoma (excl spinnerets) }\end{array}$ & $\begin{array}{l}\text { inferred, hunting } \\
\text { spiders }\end{array}$ \\
\hline Araneae & Sparassidae & Fresh mass & $M=\exp (a+b * \log (L))$ & -2.108 & 3.017 & 0.67 & 36 & (Höfer \& Ott, 2009) & $\begin{array}{l}\text { edge of prosoma (without chelicerae) to edge of } \\
\text { opisthosoma (excl spinnerets) }\end{array}$ & $\begin{array}{l}\text { inferred, hunting } \\
\text { spiders }\end{array}$ \\
\hline Araneae & Stenochilidae & Fresh mass & $M=\exp (a+b * \log (L))$ & -2.108 & 3.017 & 0.67 & 36 & (Höfer \& Ott, 2009) & $\begin{array}{l}\text { edge of prosoma (without chelicerae) to edge of } \\
\text { opisthosoma (excl spinnerets) }\end{array}$ & $\begin{array}{l}\text { inferred, hunting } \\
\text { spiders }\end{array}$ \\
\hline
\end{tabular}




\begin{tabular}{|c|c|c|c|c|c|c|c|c|c|c|}
\hline Araneae & Symphytognathidae & Fresh mass & $\mathrm{M}=\exp (\mathrm{a}+\mathrm{b} * \log (\mathrm{L}))$ & -1.784 & 2.255 & 0.56 & 10.67 & (Höfer \& Ott, 2009) & $\begin{array}{l}\text { edge of prosoma (without chelicerae) to edge of } \\
\text { opisthosoma (excl spinnerets) }\end{array}$ & $\begin{array}{l}\text { inferred, web- } \\
\text { building }\end{array}$ \\
\hline Araneae & Telemidae & Fresh mass & $\mathrm{M}=\exp (\mathrm{a}+\mathrm{b} * \log (\mathrm{L}))$ & -1.784 & 2.255 & 0.56 & 10.67 & (Höfer \& Ott, 2009) & $\begin{array}{l}\text { edge of prosoma (without chelicerae) to edge of } \\
\text { opisthosoma (excl spinnerets) }\end{array}$ & $\begin{array}{l}\text { inferred, web- } \\
\text { building }\end{array}$ \\
\hline Araneae & Tetrablemmidae & Fresh mass & $\mathrm{M}=\exp (\mathrm{a}+\mathrm{b} * \log (\mathrm{L}))$ & -2.039 & 2.666 & 0.67 & 2.5 & (Höfer \& Ott, 2009) & $\begin{array}{l}\text { edge of prosoma (without chelicerae) to edge of } \\
\text { opisthosoma (excl spinnerets) }\end{array}$ & inferred, Oonopidae \\
\hline Araneae & Tetragnathidae & Fresh mass & $\mathrm{M}=\exp (\mathrm{a}+\mathrm{b} * \log (\mathrm{L}))$ & -2.615 & 2.574 & 3.50 & 9.00 & (Edwards, 1996) & clypeus to tip of spinnerets & Group specific \\
\hline Araneae & Therididae & Fresh mass & $\mathrm{M}=\exp (\mathrm{a}+\mathrm{b} * \log (\mathrm{L}))$ & -1.577 & 2.907 & 1.50 & 7.50 & (Edwards, 1996) & clypeus to tip of spinnerets & Group specific \\
\hline Araneae & Theridiosomatidae & Fresh mass & $\mathrm{M}=\exp (\mathrm{a}+\mathrm{b} * \log (\mathrm{L}))$ & -1.784 & 2.255 & 0.56 & 10.67 & (Höfer \& Ott, 2009) & $\begin{array}{l}\text { edge of prosoma (without chelicerae) to edge of } \\
\text { opisthosoma (excl spinnerets) }\end{array}$ & $\begin{array}{l}\text { inferred, web- } \\
\text { building }\end{array}$ \\
\hline Araneae & Thomisidae & Fresh mass & $\mathrm{M}=\exp (\mathrm{a}+\mathrm{b} * \log (\mathrm{L}))$ & -1.644 & 2.973 & 1.80 & 8.00 & (Edwards, 1996) & clypeus to tip of spinnerets & Group specific \\
\hline Araneae & Uloboridae & Fresh mass & $\mathrm{M}=\exp (\mathrm{a}+\mathrm{b} * \log (\mathrm{L}))$ & -1.784 & 2.255 & 0.56 & 10.67 & (Höfer \& Ott, 2009) & $\begin{array}{l}\text { edge of prosoma (without chelicerae) to edge of } \\
\text { opisthosoma (excl spinnerets) }\end{array}$ & $\begin{array}{l}\text { inferred, web- } \\
\text { building }\end{array}$ \\
\hline Araneae & Unidentifiable $<1.8$ & Fresh mass & $\mathrm{M}=\exp (\mathrm{a}+\mathrm{b} * \log (\mathrm{L}))$ & -1.958 & 2.746 & 0.56 & 2.5 & (Höfer \& Ott, 2009) & $\begin{array}{l}\text { edge of prosoma (without chelicerae) to edge of } \\
\text { opisthosoma (excl spinnerets) }\end{array}$ & $\begin{array}{l}\text { inferred, Araneae < } \\
2.5 \mathrm{~mm}\end{array}$ \\
\hline Araneae & Unidentifiable $>1.8$ & fresh mass & $\mathrm{M}=\exp (\mathrm{a}+\mathrm{b} * \log (\mathrm{L}))$ & -1.844 & 2.711 & 1.8 & 21.5 & (Edwards, 1996) & clypeus to tip of spinnerets & $\begin{array}{l}\text { inferred, spiders } \\
\text { random sample }\end{array}$ \\
\hline Araneae & Zodariidae & Fresh mass & $\mathrm{M}=\exp (\mathrm{a}+\mathrm{b} * \log (\mathrm{L}))$ & -2.108 & 3.017 & 0.67 & 36 & (Höfer \& Ott, 2009) & $\begin{array}{l}\text { edge of prosoma (without chelicerae) to edge of } \\
\text { opisthosoma (excl spinnerets) }\end{array}$ & $\begin{array}{l}\text { inferred, hunting } \\
\text { spiders }\end{array}$ \\
\hline Archaeognatha & All & Dry mass & $\mathrm{M}=\exp (\mathrm{a}+\mathrm{b} * \log (\mathrm{L}))$ & -3.628 & 2.494 & 2.13 & 54.51 & (Sample, Cooper, Greer, \& Whitmore, 1993) & $\begin{array}{l}\text { From frons to tip of abdomen excluding } \\
\text { appendages }\end{array}$ & $\begin{array}{l}\text { inferred, all insect } \\
\text { taxa }\end{array}$ \\
\hline Blattodea & Blaberidae & Dry mass & $\mathrm{M}=\exp (\mathrm{a}+\mathrm{b} * \log (\mathrm{L}))$ & -3.980 & 2.760 & 2.20 & 14.00 & (Wardhaugh, 2013) & $\begin{array}{l}\text { front of labrum to tip of abdomen (excl. cerci or } \\
\text { ovipositors) or tip of elytra (longest) }\end{array}$ & inferred, Blattodea \\
\hline Blattodea & Blattellidae & Dry mass & $\mathrm{M}=\exp (\mathrm{a}+\mathrm{b} * \log (\mathrm{L}))$ & -3.980 & 2.760 & 2.20 & 14.00 & (Wardhaugh, 2013) & $\begin{array}{l}\text { front of labrum to tip of abdomen (excl. cerci or } \\
\text { ovipositors) or tip of elytra (longest) }\end{array}$ & inferred, Blattodea \\
\hline Blattodea & Blattidae & Dry mass & $\mathrm{M}=\exp (\mathrm{a}+\mathrm{b} * \log (\mathrm{L}))$ & -3.980 & 2.760 & 2.20 & 14.00 & (Wardhaugh, 2013) & $\begin{array}{l}\text { front of labrum to tip of abdomen (excl. cerci or } \\
\text { ovipositors) or tip of elytra (longest) }\end{array}$ & inferred, Blattodea \\
\hline Blattodea & Unidentifiable & Dry mass & $\mathrm{M}=\exp (\mathrm{a}+\mathrm{b} * \log (\mathrm{L}))$ & -3.980 & 2.760 & 2.20 & 14.00 & (Wardhaugh, 2013) & $\begin{array}{l}\text { front of labrum to tip of abdomen (excl. cerci or } \\
\text { ovipositors) or tip of elytra (longest) }\end{array}$ & inferred, Blattodea \\
\hline Chilopoda & Ballophilidae & Dry mass & $\mathrm{M}=\exp (\mathrm{a}+\mathrm{b} * \log (\mathrm{L}))$ & -4.049 & 2.18 & 4 & 47 & (Gowing \& Recher, 1984) & not mentioned & inferred, Chilopoda \\
\hline Chilopoda & Cryptopidae & Dry mass & $M=\exp (a+b * \log (L))$ & -4.049 & 2.18 & 4 & 47 & (Gowing \& Recher, 1984) & not mentioned & inferred, Chilopoda \\
\hline Chilopoda & Henicopidae & Dry mass & $M=\exp (a+b * \log (L))$ & -4.049 & 2.18 & 4 & 47 & (Gowing \& Recher, 1984) & not mentioned & inferred, Chilopoda \\
\hline Chilopoda & Lithobiomorpha & Dry mass & $M=\exp (a+b * \log (L))$ & -4.049 & 2.18 & 4 & 47 & (Gowing \& Recher, 1984) & not mentioned & inferred, Chilopoda \\
\hline Chilopoda & Mecistocephalidae & Dry mass & $\mathrm{M}=\exp (\mathrm{a}+\mathrm{b} * \log (\mathrm{L}))$ & -4.049 & 2.18 & 4 & 47 & (Gowing \& Recher, 1984) & not mentioned & inferred, Chilopoda \\
\hline
\end{tabular}




\begin{tabular}{|c|c|c|c|c|c|c|c|c|c|c|}
\hline Chilopoda & Scolopendridae & Dry mass & $M=\exp (a+b * \log (L))$ & -4.049 & 2.18 & 4 & 47 & (Gowing \& Recher, 1984) & not mentioned & inferred, Chilopoda \\
\hline Chilopoda & Unidentifiable & Dry mass & $M=\exp (a+b * \log (L))$ & -4.049 & 2.18 & 4 & 47 & (Gowing \& Recher, 1984) & not mentioned & inferred, Chilopoda \\
\hline Coleoptera & Anobiidae & Dry mass & $\mathrm{M}=\exp (\mathrm{a}+\mathrm{b} * \log (\mathrm{L}))$ & -3.247 & 2.492 & 3.34 & 34.82 & (Sample, Cooper, Greer, \& Whitmore, 1993) & $\begin{array}{l}\text { From frons to tip of abdomen excluding } \\
\text { appendages }\end{array}$ & inferred, Coleoptera \\
\hline Coleoptera & Anthicidae & Dry mass & $\mathrm{M}=\exp (\mathrm{a}+\mathrm{b} * \log (\mathrm{L}))$ & -3.247 & 2.492 & 3.34 & 34.82 & (Sample, Cooper, Greer, \& Whitmore, 1993) & $\begin{array}{l}\text { From frons to tip of abdomen excluding } \\
\text { appendages }\end{array}$ & inferred, Coleoptera \\
\hline Coleoptera & Bostrichidae & Dry mass & $\mathrm{M}=\exp (\mathrm{a}+\mathrm{b} * \log (\mathrm{L}))$ & -3.247 & 2.492 & 3.34 & 34.82 & (Sample, Cooper, Greer, \& Whitmore, 1993) & $\begin{array}{l}\text { From frons to tip of abdomen excluding } \\
\text { appendages }\end{array}$ & inferred, Coleoptera \\
\hline Coleoptera & Byrrhidae & Dry mass & $\mathrm{M}=\exp (\mathrm{a}+\mathrm{b} * \log (\mathrm{L}))$ & -3.247 & 2.492 & 3.34 & 34.82 & (Sample, Cooper, Greer, \& Whitmore, 1993) & $\begin{array}{l}\text { From frons to tip of abdomen excluding } \\
\text { appendages }\end{array}$ & inferred, Coleoptera \\
\hline Coleoptera & Carabidae & Dry mass & $\mathrm{M}=\mathrm{a} * \mathrm{~L}^{\wedge} \mathrm{b}$ & 0.0237 & 2.7054 & 2.88 & 24 & (Lang, Krooss \& Stumpf, 1997) & $\begin{array}{l}\text { Measured from anterior tip of head to posterior of } \\
\text { abdomen excluding any appendages }\end{array}$ & Group specific \\
\hline Coleoptera & Cerylonidae & Dry mass & $\mathrm{M}=\exp (\mathrm{a}+\mathrm{b} * \log (\mathrm{L}))$ & -3.247 & 2.492 & 3.34 & 34.82 & (Sample, Cooper, Greer, \& Whitmore, 1993) & $\begin{array}{l}\text { From frons to tip of abdomen excluding } \\
\text { appendages }\end{array}$ & inferred, Coleoptera \\
\hline Coleoptera & Chelonariidae & Dry mass & $\mathrm{M}=\exp (\mathrm{a}+\mathrm{b} * \log (\mathrm{L}))$ & -3.247 & 2.492 & 3.34 & 34.82 & (Sample, Cooper, Greer, \& Whitmore, 1993) & $\begin{array}{l}\text { From frons to tip of abdomen excluding } \\
\text { appendages }\end{array}$ & inferred, Coleoptera \\
\hline Coleoptera & Chrysomelidae & Dry mass & $\mathrm{M}=\exp (\mathrm{a}+\mathrm{b} * \log (\mathrm{L}))$ & -2.427 & 2.171 & 3.34 & 7.84 & (Sample, Cooper, Greer, \& Whitmore, 1993) & $\begin{array}{l}\text { From frons to tip of abdomen excluding } \\
\text { appendages }\end{array}$ & Group specific \\
\hline Coleoptera & Ciidae & Dry mass & $\mathrm{M}=\exp (\mathrm{a}+\mathrm{b} * \log (\mathrm{L}))$ & -3.247 & 2.492 & 3.34 & 34.82 & (Sample, Cooper, Greer, \& Whitmore, 1993) & $\begin{array}{l}\text { From frons to tip of abdomen excluding } \\
\text { appendages }\end{array}$ & inferred, Coleoptera \\
\hline Coleoptera & Cleridae & Dry mass & $\mathrm{M}=\exp (\mathrm{a}+\mathrm{b} * \log (\mathrm{L}))$ & -3.247 & 2.492 & 3.34 & 34.82 & (Sample, Cooper, Greer, \& Whitmore, 1993) & $\begin{array}{l}\text { From frons to tip of abdomen excluding } \\
\text { appendages }\end{array}$ & inferred, Coleoptera \\
\hline Coleoptera & Coccinellidae & Dry mass & $\mathrm{M}=\exp (\mathrm{a}+\mathrm{b} * \log (\mathrm{L}))$ & -3.247 & 2.492 & 3.34 & 34.82 & (Sample, Cooper, Greer, \& Whitmore, 1993) & $\begin{array}{l}\text { From frons to tip of abdomen excluding } \\
\text { appendages }\end{array}$ & inferred, Coleoptera \\
\hline Coleoptera & Colydiidae & Dry mass & $\mathrm{M}=\exp (\mathrm{a}+\mathrm{b} * \log (\mathrm{L}))$ & -3.247 & 2.492 & 3.34 & 34.82 & (Sample, Cooper, Greer, \& Whitmore, 1993) & $\begin{array}{l}\text { From frons to tip of abdomen excluding } \\
\text { appendages }\end{array}$ & inferred, Coleoptera \\
\hline Coleoptera & Curculionidae & Dry mass & $\mathrm{M}=\exp (\mathrm{a}+\mathrm{b} * \log (\mathrm{L}))$ & -3.247 & 2.492 & 3.34 & 34.82 & (Sample, Cooper, Greer, \& Whitmore, 1993) & $\begin{array}{l}\text { From frons to tip of abdomen excluding } \\
\text { appendages }\end{array}$ & inferred, Coleoptera \\
\hline Coleoptera & Dermestidae & Dry mass & $\mathrm{M}=\exp (\mathrm{a}+\mathrm{b} * \log (\mathrm{L}))$ & -3.247 & 2.492 & 3.34 & 34.82 & (Sample, Cooper, Greer, \& Whitmore, 1993) & $\begin{array}{l}\text { From frons to tip of abdomen excluding } \\
\text { appendages }\end{array}$ & inferred, Coleoptera \\
\hline Coleoptera & Discolomidae & Dry mass & $\mathrm{M}=\exp (\mathrm{a}+\mathrm{b} * \log (\mathrm{L}))$ & -3.247 & 2.492 & 3.34 & 34.82 & (Sample, Cooper, Greer, \& Whitmore, 1993) & $\begin{array}{l}\text { From frons to tip of abdomen excluding } \\
\text { appendages }\end{array}$ & inferred, Coleoptera \\
\hline Coleoptera & Elateridae & Dry mass & $\mathrm{M}=\mathrm{a} * \mathrm{~L}^{\wedge} \mathrm{b}$ & 0.0138 & 2.595 & 1.65 & 10.3 & (Gruner, 2003) & $\begin{array}{l}\text { tip of abdomen to end of head or carapace, excl. } \\
\text { any appendages }\end{array}$ & $\begin{array}{l}\text { inferred, slender } \\
\text { beetles }\end{array}$ \\
\hline Coleoptera & Endomychidae & Dry mass & $M=\exp (a+b * \log (L))$ & -3.247 & 2.492 & 3.34 & 34.82 & (Sample, Cooper, Greer, \& Whitmore, 1993) & $\begin{array}{l}\text { From frons to tip of abdomen excluding } \\
\text { appendages }\end{array}$ & inferred, Coleoptera \\
\hline Coleoptera & Histeridae & Dry mass & $\mathrm{M}=\exp (\mathrm{a}+\mathrm{b} * \log (\mathrm{L}))$ & -3.247 & 2.492 & 3.34 & 34.82 & (Sample, Cooper, Greer, \& Whitmore, 1993) & $\begin{array}{l}\text { From frons to tip of abdomen excluding } \\
\text { appendages }\end{array}$ & inferred, Coleoptera \\
\hline Coleoptera & Hydrophilidae & Dry mass & $\mathrm{M}=\exp (\mathrm{a}+\mathrm{b} * \log (\mathrm{L}))$ & -3.247 & 2.492 & 3.34 & 34.82 & (Sample, Cooper, Greer, \& Whitmore, 1993) & $\begin{array}{l}\text { From frons to tip of abdomen excluding } \\
\text { appendages }\end{array}$ & inferred, Coleoptera \\
\hline
\end{tabular}




\begin{tabular}{|c|c|c|c|c|c|c|c|c|c|c|}
\hline Coleoptera & Languriidae & Dry mass & $M=\exp (a+b * \log (L))$ & -3.247 & 2.492 & 3.34 & 34.82 & (Sample, Cooper, Greer, \& Whitmore, 1993) & $\begin{array}{l}\text { From frons to tip of abdomen excluding } \\
\text { appendages }\end{array}$ & inferred, Coleoptera \\
\hline Coleoptera & Larvae & Dry mass & $\mathrm{M}=\mathrm{a} * \mathrm{~L}^{\wedge} \mathrm{b}$ & 0.0035 & 2.4033 & 1.5 & 25.27 & (Lang, Krooss, \& Stumpf, 1997) & $\begin{array}{l}\text { Measured from anterior tip of head to posterior of } \\
\text { abdomen excluding any appendages }\end{array}$ & inferred, Coleoptera \\
\hline Coleoptera & Leiodidae & Dry mass & $\mathrm{M}=\exp (\mathrm{a}+\mathrm{b} * \log (\mathrm{L}))$ & -3.247 & 2.492 & 3.34 & 34.82 & (Sample, Cooper, Greer, \& Whitmore, 1993) & $\begin{array}{l}\text { From frons to tip of abdomen excluding } \\
\text { appendages }\end{array}$ & inferred, Coleoptera \\
\hline Coleoptera & Lucanidae & Dry mass & $M=\exp (a+b * \log (L))$ & -3.247 & 2.492 & 3.34 & 34.82 & (Sample, Cooper, Greer, \& Whitmore, 1993) & $\begin{array}{l}\text { From frons to tip of abdomen excluding } \\
\text { appendages }\end{array}$ & inferred, Coleoptera \\
\hline Coleoptera & Melyridae & Dry mass & $M=\exp (a+b * \log (L))$ & -3.247 & 2.492 & 3.34 & 34.82 & (Sample, Cooper, Greer, \& Whitmore, 1993) & $\begin{array}{l}\text { From frons to tip of abdomen excluding } \\
\text { appendages }\end{array}$ & inferred, Coleoptera \\
\hline Coleoptera & Mordellidae & Dry mass & $\mathrm{M}=\exp (\mathrm{a}+\mathrm{b} * \log (\mathrm{L}))$ & -3.247 & 2.492 & 3.34 & 34.82 & (Sample, Cooper, Greer, \& Whitmore, 1993) & $\begin{array}{l}\text { From frons to tip of abdomen excluding } \\
\text { appendages }\end{array}$ & inferred, Coleoptera \\
\hline Coleoptera & Mycetophagidae & Dry mass & $\mathrm{M}=\exp (\mathrm{a}+\mathrm{b} * \log (\mathrm{L}))$ & -3.247 & 2.492 & 3.34 & 34.82 & (Sample, Cooper, Greer, \& Whitmore, 1993) & $\begin{array}{l}\text { From frons to tip of abdomen excluding } \\
\text { appendages }\end{array}$ & inferred, Coleoptera \\
\hline Coleoptera & Pselaphidae & Dry mass & $M=\exp (a+b * \log (L))$ & -3.247 & 2.492 & 3.34 & 34.82 & (Sample, Cooper, Greer, \& Whitmore, 1993) & $\begin{array}{l}\text { From frons to tip of abdomen excluding } \\
\text { appendages }\end{array}$ & inferred, Coleoptera \\
\hline Coleoptera & Ptiliidae & Dry mass & $\mathrm{M}=\exp (\mathrm{a}+\mathrm{b} * \log (\mathrm{L}))$ & -3.247 & 2.492 & 3.34 & 34.82 & (Sample, Cooper, Greer, \& Whitmore, 1993) & $\begin{array}{l}\text { From frons to tip of abdomen excluding } \\
\text { appendages }\end{array}$ & inferred, Coleoptera \\
\hline Coleoptera & Scarabaeidae & Dry mass & $M=\exp (a+b * \log (L))$ & -2.448 & 2.494 & 4.24 & 24.79 & (Sample, Cooper, Greer, \& Whitmore, 1993) & $\begin{array}{l}\text { From frons to tip of abdomen excluding } \\
\text { appendages }\end{array}$ & Group specific \\
\hline Coleoptera & Scydmaenidae & Dry mass & $M=\exp (a+b * \log (L))$ & -3.247 & 2.492 & 3.34 & 34.82 & (Sample, Cooper, Greer, \& Whitmore, 1993) & $\begin{array}{l}\text { From frons to tip of abdomen excluding } \\
\text { appendages }\end{array}$ & inferred, Coleoptera \\
\hline Coleoptera & Silvanidae & Dry mass & $M=a * L^{\wedge} b$ & 0.0138 & 2.595 & 1.65 & 10.3 & (Gruner, 2003) & $\begin{array}{l}\text { tip of abdomen to end of head or carapace, excl. } \\
\text { any appendages }\end{array}$ & $\begin{array}{l}\text { inferred, slender } \\
\text { beetles }\end{array}$ \\
\hline Coleoptera & Staphylinidae & Dry mass & $M=a * L^{\wedge} b$ & 0.0134 & 2.26 & 2.2 & 13.6 & (Lang, Krooss, \& Stumpf, 1997) & $\begin{array}{l}\text { Measured from anterior tip of head to posterior of } \\
\text { abdomen excluding any appendages }\end{array}$ & Group specific \\
\hline Coleoptera & Tenebrionidae & Dry mass & $\mathrm{M}=\exp (\mathrm{a}+\mathrm{b} * \log (\mathrm{L}))$ & -0.043 & 1.2 & 5.65 & 13.39 & (Sample, Cooper, Greer, \& Whitmore, 1993) & $\begin{array}{l}\text { From frons to tip of abdomen excluding } \\
\text { appendages }\end{array}$ & Group specific \\
\hline Coleoptera & Throscidae & Dry mass & $M=\exp (a+b * \log (L))$ & -3.247 & 2.492 & 3.34 & 34.82 & (Sample, Cooper, Greer, \& Whitmore, 1993) & $\begin{array}{l}\text { From frons to tip of abdomen excluding } \\
\text { appendages }\end{array}$ & inferred, Coleoptera \\
\hline Coleoptera & Trogossitidae & Dry mass & $M=\exp (a+b * \log (L))$ & -3.247 & 2.492 & 3.34 & 34.82 & (Sample, Cooper, Greer, \& Whitmore, 1993) & $\begin{array}{l}\text { From frons to tip of abdomen excluding } \\
\text { appendages }\end{array}$ & inferred, Coleoptera \\
\hline Coleoptera & Unidentifiable & Dry mass & $M=\exp (a+b * \log (L))$ & -3.247 & 2.492 & 3.34 & 34.82 & (Sample, Cooper, Greer, \& Whitmore, 1993) & $\begin{array}{l}\text { From frons to tip of abdomen excluding } \\
\text { appendages }\end{array}$ & inferred, Coleoptera \\
\hline Coleoptera & Zopheridae & Dry mass & $\mathrm{M}=\exp (\mathrm{a}+\mathrm{b} * \log (\mathrm{L}))$ & -3.247 & 2.492 & 3.34 & 34.82 & (Sample, Cooper, Greer, \& Whitmore, 1993) & $\begin{array}{l}\text { From frons to tip of abdomen excluding } \\
\text { appendages }\end{array}$ & inferred, Coleoptera \\
\hline Dermaptera & Anisolabididae & Dry mass & $\mathrm{M}=\exp (\mathrm{a}+\mathrm{b} * \log (\mathrm{L}))$ & -3.628 & 2.494 & 2.13 & 54.51 & (Sample, Cooper, Greer, \& Whitmore, 1993) & $\begin{array}{l}\text { From frons to tip of abdomen excluding } \\
\text { appendages }\end{array}$ & $\begin{array}{l}\text { inferred, all insect } \\
\text { taxa }\end{array}$ \\
\hline Dermaptera & Forficulidae & Dry mass & $\mathrm{M}=\exp (\mathrm{a}+\mathrm{b} * \log (\mathrm{L}))$ & -3.628 & 2.494 & 2.13 & 54.51 & (Sample, Cooper, Greer, \& Whitmore, 1993) & $\begin{array}{l}\text { From frons to tip of abdomen excluding } \\
\text { appendages }\end{array}$ & $\begin{array}{l}\text { inferred, all insect } \\
\text { taxa }\end{array}$ \\
\hline Diplopoda & Chordeumatida & Dry mass & $M=\exp (a+b * \log (L))$ & -4.591 & 2.543 & 11.0 & 47.0 & (Gowing \& Recher, 1984) & not mentioned & inferred, Diplopoda \\
\hline
\end{tabular}




\begin{tabular}{|c|c|c|c|c|c|c|c|c|c|c|c|}
\hline Diplopoda & Glomerida & & Dry mass & $M=\exp (a+b * \log (L))$ & -4.591 & 2.543 & 11.0 & 47.0 & (Gowing \& Recher, 1984) & not mentioned & inferred, Diplopoda \\
\hline Diplopoda & Polidesmatidae & & Dry mass & $\mathrm{M}=\exp (\mathrm{a}+\mathrm{b} * \log (\mathrm{L}))$ & -4.591 & 2.543 & 11.0 & 47.0 & (Gowing \& Recher, 1984) & not mentioned & inferred, Diplopoda \\
\hline Diplopoda & Polydesmatida & & Dry mass & $M=\exp (a+b * \log (L))$ & -4.591 & 2.543 & 11.0 & 47.0 & (Gowing \& Recher, 1984) & not mentioned & inferred, Diplopoda \\
\hline Diplopoda & Polydesmida & & Dry mass & $\mathrm{M}=\exp (\mathrm{a}+\mathrm{b} * \log (\mathrm{L}))$ & -4.591 & 2.543 & 11.0 & 47.0 & (Gowing \& Recher, 1984) & not mentioned & inferred, Diplopoda \\
\hline Diplopoda & Polydesmidae & & Dry mass & $M=\exp (a+b * \log (L))$ & -4.591 & 2.543 & 11.0 & 47.0 & (Gowing \& Recher, 1984) & not mentioned & inferred, Diplopoda \\
\hline Diplopoda & Polyxenida & & Dry mass & $\mathrm{M}=\exp (\mathrm{a}+\mathrm{b} * \log (\mathrm{L}))$ & -4.591 & 2.543 & 11.0 & 47.0 & (Gowing \& Recher, 1984) & not mentioned & inferred, Diplopoda \\
\hline Diplopoda & Siphonophorida & & Dry mass & $M=\exp (a+b * \log (L))$ & -4.591 & 2.543 & 11.0 & 47.0 & (Gowing \& Recher, 1984) & not mentioned & inferred, Diplopoda \\
\hline Diplopoda & Spirobolida & & Dry mass & $M=\exp (a+b * \log (L))$ & -4.591 & 2.543 & 11.0 & 47.0 & (Gowing \& Recher, 1984) & not mentioned & inferred, Diplopoda \\
\hline Diplura & Heterojapygidae & & Dry mass & $\mathrm{M}=\mathrm{a} *(\mathrm{~L})^{\wedge} \mathrm{b}$ & 0.034 & 2.191 & 0.9 & 17.6 & (Gruner, 2003) & $\begin{array}{l}\text { tip of abdomen to end of head or carapace, excl. } \\
\text { any appendages }\end{array}$ & $\begin{array}{l}\text { inferred, general } \\
\text { arthropod }\end{array}$ \\
\hline Diptera & Larvae & & Dry mass & $\mathrm{M}=\mathrm{a} *(\mathrm{~L})^{\wedge} \mathrm{b}$ & 0.029 & 1.73 & 1.7 & 16.65 & (Gruner, 2003) & $\begin{array}{l}\text { tip of abdomen to end of head or carapace, excl. } \\
\text { any appendages }\end{array}$ & $\begin{array}{l}\text { inferred, holomet. } \\
\text { Larvae }\end{array}$ \\
\hline Diptera & Adults & & Dry mass & $M=a *(L)^{\wedge} b$ & 0.0153 & 2.573 & 1.75 & 8.6 & (Gruner, 2003) & $\begin{array}{l}\text { tip of abdomen to end of head or carapace, excl. } \\
\text { any appendages }\end{array}$ & $\begin{array}{l}\text { Group specific, } \\
\text { Diptera adult }\end{array}$ \\
\hline Diptera & Agromyzidae & Larvae & Dry mass & $\mathrm{M}=\mathrm{a} *(\mathrm{~L})^{\wedge} \mathrm{b}$ & 0.029 & 1.73 & 1.7 & 16.65 & (Gruner, 2003) & $\begin{array}{l}\text { tip of abdomen to end of head or carapace, excl. } \\
\text { any appendages }\end{array}$ & $\begin{array}{l}\text { inferred, holomet. } \\
\text { Larvae }\end{array}$ \\
\hline Diptera & Cecidomyiidae & $\mathrm{A} / \mathrm{L}$ & Dry mass & $M=a *(L)^{\wedge} b$ & 0.035 & 2.173 & 0.9 & 17.6 & (Gruner, 2003) & $\begin{array}{l}\text { tip of abdomen to end of head or carapace, excl. } \\
\text { any appendages }\end{array}$ & $\begin{array}{l}\text { inferred, all insect } \\
\text { taxa }\end{array}$ \\
\hline Diptera & Ceratopogonidae & Larvae & Dry mass & $M=a *(L)^{\wedge} b$ & 0.029 & 1.73 & 1.7 & 16.65 & (Gruner, 2003) & $\begin{array}{l}\text { tip of abdomen to end of head or carapace, excl. } \\
\text { any appendages }\end{array}$ & $\begin{array}{l}\text { inferred, holomet. } \\
\text { Larvae }\end{array}$ \\
\hline Diptera & Chironomidae & Larvae & Dry mass & $\mathrm{M}=\mathrm{a} *(\mathrm{~L})^{\wedge} \mathrm{b}$ & 0.029 & 1.73 & 1.7 & 16.65 & (Gruner, 2003) & $\begin{array}{l}\text { tip of abdomen to end of head or carapace, excl. } \\
\text { any appendages }\end{array}$ & $\begin{array}{l}\text { inferred, holomet. } \\
\text { Larvae }\end{array}$ \\
\hline Diptera & Drosophilidae & Larvae & Dry mass & $M=a *(L)^{\wedge} b$ & 0.029 & 1.73 & 1.7 & 16.65 & (Gruner, 2003) & $\begin{array}{l}\text { tip of abdomen to end of head or carapace, excl. } \\
\text { any appendages }\end{array}$ & $\begin{array}{l}\text { inferred, holomet. } \\
\text { Larvae }\end{array}$ \\
\hline Diptera & Muscidae & Adult & Dry mass & $\mathrm{M}=\mathrm{a} *(\mathrm{~L})^{\wedge} \mathrm{b}$ & 0.0153 & 2.573 & 1.75 & 8.6 & (Gruner, 2003) & $\begin{array}{l}\text { tip of abdomen to end of head or carapace, excl. } \\
\text { any appendages }\end{array}$ & $\begin{array}{l}\text { Group specific, } \\
\text { Diptera adult }\end{array}$ \\
\hline Diptera & Mycetophilidae & Larvae & Dry mass & $\mathrm{M}=\mathrm{a} *(\mathrm{~L})^{\wedge} \mathrm{b}$ & 0.029 & 1.73 & 1.7 & 16.65 & (Gruner, 2003) & $\begin{array}{l}\text { tip of abdomen to end of head or carapace, excl. } \\
\text { any appendages }\end{array}$ & $\begin{array}{l}\text { inferred, holomet. } \\
\text { Larvae }\end{array}$ \\
\hline Diptera & Phoridae & Adult & Dry mass & $\mathrm{M}=\mathrm{a} *(\mathrm{~L})^{\wedge} \mathrm{b}$ & 0.0153 & 2.573 & 1.75 & 8.6 & (Gruner, 2003) & $\begin{array}{l}\text { tip of abdomen to end of head or carapace, excl. } \\
\text { any appendages }\end{array}$ & $\begin{array}{l}\text { Group specific, } \\
\text { Diptera adult }\end{array}$ \\
\hline Diptera & Pipunculidae & Larvae & Dry mass & $\mathrm{M}=\mathrm{a} *(\mathrm{~L})^{\wedge} \mathrm{b}$ & 0.029 & 1.73 & 1.7 & 16.65 & (Gruner, 2003) & $\begin{array}{l}\text { tip of abdomen to end of head or carapace, excl. } \\
\text { any appendages }\end{array}$ & $\begin{array}{l}\text { inferred, holomet. } \\
\text { Larvae }\end{array}$ \\
\hline Diptera & Sciaridae & Adult & Dry mass & $\mathrm{M}=\mathrm{a} *(\mathrm{~L})^{\wedge} \mathrm{b}$ & 0.0153 & 2.573 & 1.75 & 8.6 & (Gruner, 2003) & $\begin{array}{l}\text { tip of abdomen to end of head or carapace, excl. } \\
\text { any appendages }\end{array}$ & $\begin{array}{l}\text { Group specific, } \\
\text { Diptera adult }\end{array}$ \\
\hline
\end{tabular}




\begin{tabular}{|c|c|c|c|c|c|c|c|c|c|c|c|}
\hline Diptera & Simuliidae & Adult & Dry mass & $M=a *(L)^{\wedge} b$ & 0.0153 & 2.573 & 1.75 & 8.6 & (Gruner, 2003) & $\begin{array}{l}\text { tip of abdomen to end of head or carapace, excl. } \\
\text { any appendages }\end{array}$ & $\begin{array}{l}\text { Group specific, } \\
\text { Diptera adult }\end{array}$ \\
\hline Diptera & Syrphidae & Larvae & Dry mass & $M=a *(L)^{\wedge} b$ & 0.029 & 1.73 & 1.7 & 16.65 & (Gruner, 2003) & $\begin{array}{l}\text { tip of abdomen to end of head or carapace, excl. } \\
\text { any appendages }\end{array}$ & $\begin{array}{l}\text { inferred, holomet. } \\
\text { Larvae }\end{array}$ \\
\hline Diptera & Tachinidae & Adult & Dry mass & $M=a *(L)^{\wedge} b$ & 0.0153 & 2.573 & 1.75 & 8.6 & (Gruner, 2003) & $\begin{array}{l}\text { tip of abdomen to end of head or carapace, excl. } \\
\text { any appendages }\end{array}$ & $\begin{array}{l}\text { Group specific, } \\
\text { Diptera adult }\end{array}$ \\
\hline Diptera & Tephritidae & Larvae & Dry mass & $M=a *(L)^{\wedge} b$ & 0.029 & 1.73 & 1.7 & 16.65 & (Gruner, 2003) & $\begin{array}{l}\text { tip of abdomen to end of head or carapace, excl. } \\
\text { any appendages }\end{array}$ & $\begin{array}{l}\text { inferred, holomet. } \\
\text { Larvae }\end{array}$ \\
\hline Diptera & Thaumaleidae & $\mathrm{A} / \mathrm{L}$ & Dry mass & $M=a *(L)^{\wedge} b$ & 0.035 & 2.173 & 0.9 & 17.6 & (Gruner, 2003) & $\begin{array}{l}\text { tip of abdomen to end of head or carapace, excl. } \\
\text { any appendages }\end{array}$ & $\begin{array}{l}\text { inferred, all insect } \\
\text { taxa }\end{array}$ \\
\hline Gastropoda & All & & Dry mass & $M=\exp (a+b * \log (L * W))$ & -2.75 & 1.59 & 2.1 & 18 & (Wardhaugh, 2013) & $\begin{array}{l}\text { front of labrum to tip of abdomen (excl. cerci or } \\
\text { ovipositors) or tip of elytra (longest) }\end{array}$ & $\begin{array}{l}\text { inferred, } \\
\text { Gastropoda }\end{array}$ \\
\hline Hemiptera & Acanthosomatidae & & Dry mass & $\mathrm{M}=\exp (\mathrm{a}+\mathrm{b} * \log (\mathrm{L}))$ & -4.784 & 3.075 & 3.2 & 40.23 & (Sample, Cooper, Greer, \& Whitmore, 1993) & $\begin{array}{l}\text { From frons to tip of abdomen excluding } \\
\text { appendages }\end{array}$ & $\begin{array}{l}\text { Group specific, } \\
\text { Hemiptera }\end{array}$ \\
\hline Hemiptera & Anthocoridae & & Dry mass & $M=\exp (a+b * \log (L))$ & -4.784 & 3.075 & 3.2 & 40.23 & (Sample, Cooper, Greer, \& Whitmore, 1993) & $\begin{array}{l}\text { From frons to tip of abdomen excluding } \\
\text { appendages }\end{array}$ & $\begin{array}{l}\text { Group specific, } \\
\text { Hemiptera }\end{array}$ \\
\hline Hemiptera & Aradidae & & Dry mass & $\mathrm{M}=\exp (\mathrm{a}+\mathrm{b} * \log (\mathrm{L}))$ & -4.784 & 3.075 & 3.2 & 40.23 & (Sample, Cooper, Greer, \& Whitmore, 1993) & $\begin{array}{l}\text { From frons to tip of abdomen excluding } \\
\text { appendages }\end{array}$ & $\begin{array}{l}\text { Group specific, } \\
\text { Hemiptera }\end{array}$ \\
\hline Hemiptera & Ceratocombidae & & Dry mass & $\mathrm{M}=\exp (\mathrm{a}+\mathrm{b} * \log (\mathrm{L}))$ & -4.784 & 3.075 & 3.2 & 40.23 & (Sample, Cooper, Greer, \& Whitmore, 1993) & $\begin{array}{l}\text { From frons to tip of abdomen excluding } \\
\text { appendages }\end{array}$ & $\begin{array}{l}\text { Group specific, } \\
\text { Hemiptera }\end{array}$ \\
\hline Hemiptera & Cicadellidae & & Dry mass & $M=\exp (a+b * \log (L))$ & -3.735 & 2.561 & 2.13 & 13.25 & (Sample, Cooper, Greer, \& Whitmore, 1993) & $\begin{array}{l}\text { From frons to tip of abdomen excluding } \\
\text { appendages }\end{array}$ & Group specific \\
\hline Hemiptera & Cimicidae & & Dry mass & $M=\exp (a+b * \log (L))$ & -4.784 & 3.075 & 3.2 & 40.23 & (Sample, Cooper, Greer, \& Whitmore, 1993) & $\begin{array}{l}\text { From frons to tip of abdomen excluding } \\
\text { appendages }\end{array}$ & inferred, Hemiptera \\
\hline Hemiptera & Cydnidae & & Dry mass & $M=\exp (a+b * \log (L))$ & -4.784 & 3.075 & 3.2 & 40.23 & (Sample, Cooper, Greer, \& Whitmore, 1993) & $\begin{array}{l}\text { From frons to tip of abdomen excluding } \\
\text { appendages }\end{array}$ & inferred, Hemiptera \\
\hline Hemiptera & Delphacidae & & Dry mass & $M=\exp (a+b * \log (L))$ & -2.823 & 2.225 & 2.13 & 13.25 & (Sample, Cooper, Greer, \& Whitmore, 1993) & $\begin{array}{l}\text { From frons to tip of abdomen excluding } \\
\text { appendages }\end{array}$ & $\begin{array}{l}\text { Inferred, } \\
\text { Homoptera }\end{array}$ \\
\hline Hemiptera & Dipsocoridae & & Dry mass & $M=\exp (a+b * \log (L))$ & -4.784 & 3.075 & 3.2 & 40.23 & (Sample, Cooper, Greer, \& Whitmore, 1993) & $\begin{array}{l}\text { From frons to tip of abdomen excluding } \\
\text { appendages }\end{array}$ & inferred, Hemiptera \\
\hline Hemiptera & Enicocephalidae & & Dry mass & $\mathrm{M}=\exp (\mathrm{a}+\mathrm{b} * \log (\mathrm{L}))$ & -4.784 & 3.075 & 3.2 & 40.23 & (Sample, Cooper, Greer, \& Whitmore, 1993) & $\begin{array}{l}\text { From frons to tip of abdomen excluding } \\
\text { appendages }\end{array}$ & inferred, Hemiptera \\
\hline Hemiptera & Eurybrachyidae & & Dry mass & $\mathrm{M}=\exp (\mathrm{a}+\mathrm{b} * \log (\mathrm{L}))$ & -2.823 & 2.225 & 2.13 & 13.25 & (Sample, Cooper, Greer, \& Whitmore, 1993) & $\begin{array}{l}\text { From frons to tip of abdomen excluding } \\
\text { appendages }\end{array}$ & $\begin{array}{l}\text { Inferred, } \\
\text { Homoptera }\end{array}$ \\
\hline Hemiptera & Hebridae & & Dry mass & $\mathrm{M}=\exp (\mathrm{a}+\mathrm{b} * \log (\mathrm{L}))$ & -4.784 & 3.075 & 3.2 & 40.23 & (Sample, Cooper, Greer, \& Whitmore, 1993) & $\begin{array}{l}\text { From frons to tip of abdomen excluding } \\
\text { appendages }\end{array}$ & inferred, Hemiptera \\
\hline Hemiptera & Hydrometridae & & Dry mass & $\mathrm{M}=\exp (\mathrm{a}+\mathrm{b} * \log (\mathrm{L}))$ & -4.784 & 3.075 & 3.2 & 40.23 & (Sample, Cooper, Greer, \& Whitmore, 1993) & $\begin{array}{l}\text { From frons to tip of abdomen excluding } \\
\text { appendages }\end{array}$ & inferred, Hemiptera \\
\hline Hemiptera & Lophopidae & & Dry mass & $\mathrm{M}=\exp (\mathrm{a}+\mathrm{b} * \log (\mathrm{L}))$ & -2.823 & 2.225 & 2.13 & 13.25 & (Sample, Cooper, Greer, \& Whitmore, 1993) & $\begin{array}{l}\text { From frons to tip of abdomen excluding } \\
\text { appendages }\end{array}$ & $\begin{array}{l}\text { Inferred, } \\
\text { Homoptera }\end{array}$ \\
\hline Hemiptera & Lygaeidae & & Dry mass & $\mathrm{M}=\exp (\mathrm{a}+\mathrm{b} * \log (\mathrm{L}))$ & -4.784 & 3.075 & 3.2 & 40.23 & (Sample, Cooper, Greer, \& Whitmore, 1993) & $\begin{array}{l}\text { From frons to tip of abdomen excluding } \\
\text { appendages }\end{array}$ & inferred, Hemiptera \\
\hline
\end{tabular}




\begin{tabular}{|c|c|c|c|c|c|c|c|c|c|c|}
\hline Hemiptera & Meenoplidae & Dry mass & $M=\exp (a+b * \log (L))$ & -2.823 & 2.225 & 2.13 & 13.25 & (Sample, Cooper, Greer, \& Whitmore, 1993) & $\begin{array}{l}\text { From frons to tip of abdomen excluding } \\
\text { appendages }\end{array}$ & $\begin{array}{l}\text { Inferred, } \\
\text { Homoptera }\end{array}$ \\
\hline Hemiptera & Membracidae & Dry mass & $\mathrm{M}=\exp (\mathrm{a}+\mathrm{b} * \log (\mathrm{L}))$ & -2.823 & 2.225 & 2.13 & 13.25 & (Sample, Cooper, Greer, \& Whitmore, 1993) & $\begin{array}{l}\text { From frons to tip of abdomen excluding } \\
\text { appendages }\end{array}$ & $\begin{array}{l}\text { Inferred, } \\
\text { Homoptera }\end{array}$ \\
\hline Hemiptera & Mesoveliidae & Dry mass & $\mathrm{M}=\exp (\mathrm{a}+\mathrm{b} * \log (\mathrm{L}))$ & -4.784 & 3.075 & 3.2 & 40.23 & (Sample, Cooper, Greer, \& Whitmore, 1993) & $\begin{array}{l}\text { From frons to tip of abdomen excluding } \\
\text { appendages }\end{array}$ & inferred, Hemiptera \\
\hline Hemiptera & Miridae & Dry mass & $\mathrm{M}=\exp (\mathrm{a}+\mathrm{b} * \log (\mathrm{L}))$ & -4.784 & 3.075 & 3.2 & 40.23 & (Sample, Cooper, Greer, \& Whitmore, 1993) & $\begin{array}{l}\text { From frons to tip of abdomen excluding } \\
\text { appendages }\end{array}$ & inferred, Hemiptera \\
\hline Hemiptera & Nabidae & Dry mass & $\mathrm{M}=\exp (\mathrm{a}+\mathrm{b} * \log (\mathrm{L}))$ & -4.784 & 3.075 & 3.2 & 40.23 & (Sample, Cooper, Greer, \& Whitmore, 1993) & $\begin{array}{l}\text { From frons to tip of abdomen excluding } \\
\text { appendages }\end{array}$ & inferred, Hemiptera \\
\hline Hemiptera & Pentatomidae & Dry mass & $\mathrm{M}=\exp (\mathrm{a}+\mathrm{b} * \log (\mathrm{L}))$ & -4.197 & 3.053 & 6.35 & 16.73 & (Sample, Cooper, Greer, \& Whitmore, 1993) & $\begin{array}{l}\text { From frons to tip of abdomen excluding } \\
\text { appendages }\end{array}$ & Group specific \\
\hline Hemiptera & Reduviidae & Dry mass & $M=\exp (a+b * \log (L))$ & -4.784 & 3.075 & 3.2 & 40.23 & (Sample, Cooper, Greer, \& Whitmore, 1993) & $\begin{array}{l}\text { From frons to tip of abdomen excluding } \\
\text { appendages }\end{array}$ & inferred, Hemiptera \\
\hline Hemiptera & Schizopteridae & Dry mass & $\mathrm{M}=\exp (\mathrm{a}+\mathrm{b} * \log (\mathrm{L}))$ & -4.784 & 3.075 & 3.2 & 40.23 & (Sample, Cooper, Greer, \& Whitmore, 1993) & $\begin{array}{l}\text { From frons to tip of abdomen excluding } \\
\text { appendages }\end{array}$ & inferred, Hemiptera \\
\hline Hemiptera & Triozidea & Dry mass & $\mathrm{M}=\exp (\mathrm{a}+\mathrm{b} * \log (\mathrm{L}))$ & -2.823 & 2.225 & 2.13 & 13.25 & (Sample, Cooper, Greer, \& Whitmore, 1993) & $\begin{array}{l}\text { From frons to tip of abdomen excluding } \\
\text { appendages }\end{array}$ & $\begin{array}{l}\text { Inferred, } \\
\text { Homoptera }\end{array}$ \\
\hline Hymenoptera & Bethylidae & Dry mass & $\mathrm{M}=\exp (\mathrm{a}+\mathrm{b} * \log (\mathrm{L}))$ & -3.336 & 2.104 & 1 & 12 & (Gowing \& Recher, 1984) & not mentioned & $\begin{array}{l}\text { inferred, Hym. excl } \\
\text { Formicidae }\end{array}$ \\
\hline Hymenoptera & Diapriidae & Dry mass & $\mathrm{M}=\exp (\mathrm{a}+\mathrm{b} * \log (\mathrm{L}))$ & -3.336 & 2.104 & 1 & 12 & (Gowing \& Recher, 1984) & not mentioned & $\begin{array}{l}\text { inferred, Hym. excl } \\
\text { Formicidae }\end{array}$ \\
\hline Hymenoptera & Eucoilidae & Dry mass & $\mathrm{M}=\exp (\mathrm{a}+\mathrm{b} * \log (\mathrm{L}))$ & -3.336 & 2.104 & 1 & 12 & (Gowing \& Recher, 1984) & not mentioned & $\begin{array}{l}\text { inferred, Hym. excl } \\
\text { Formicidae }\end{array}$ \\
\hline Hymenoptera & Eupelmidae & Dry mass & $\mathrm{M}=\exp (\mathrm{a}+\mathrm{b} * \log (\mathrm{L}))$ & -3.336 & 2.104 & 1 & 12 & (Gowing \& Recher, 1984) & not mentioned & $\begin{array}{l}\text { inferred, Hym. excl } \\
\text { Formicidae }\end{array}$ \\
\hline Hymenoptera & Figitidae & Dry mass & $\mathrm{M}=\exp (\mathrm{a}+\mathrm{b} * \log (\mathrm{L}))$ & -3.336 & 2.104 & 1 & 12 & (Gowing \& Recher, 1984) & not mentioned & $\begin{array}{l}\text { inferred, Hym. excl } \\
\text { Formicidae }\end{array}$ \\
\hline Hymenoptera & Formicidae & Dry mass & $\mathrm{M}=\exp (\mathrm{a}+\mathrm{b} * \log (\mathrm{L}))$ & -3.996 & 2.489 & 2 & 18 & (Gowing \& Recher, 1984) & not mentioned & Group specific \\
\hline Hymenoptera & Mymariidae & Dry mass & $\mathrm{M}=\exp (\mathrm{a}+\mathrm{b} * \log (\mathrm{L}))$ & -3.336 & 2.104 & 1 & 12 & (Gowing \& Recher, 1984) & not mentioned & $\begin{array}{l}\text { inferred, Hym. excl } \\
\text { Formicidae }\end{array}$ \\
\hline Hymenoptera & Scelionidae & Dry mass & $\mathrm{M}=\exp (\mathrm{a}+\mathrm{b} * \log (\mathrm{L}))$ & -3.336 & 2.104 & 1 & 12 & (Gowing \& Recher, 1984) & not mentioned & $\begin{array}{l}\text { inferred, Hym. excl } \\
\text { Formicidae }\end{array}$ \\
\hline Hymenoptera & Specidae & Dry mass & $M=\exp (a+b * \log (L))$ & -3.336 & 2.104 & 1 & 12 & (Gowing \& Recher, 1984) & not mentioned & $\begin{array}{l}\text { inferred, Hym. excl } \\
\text { Formicidae }\end{array}$ \\
\hline Hymenoptera & Trichogrammatidae & Dry mass & $\mathrm{M}=\exp (\mathrm{a}+\mathrm{b} * \log (\mathrm{L}))$ & -3.336 & 2.104 & 1 & 12 & (Gowing \& Recher, 1984) & not mentioned & $\begin{array}{l}\text { inferred, Hym. excl } \\
\text { Formicidae }\end{array}$ \\
\hline Hymenoptera & Unidentifiable & Dry mass & $\mathrm{M}=\exp (\mathrm{a}+\mathrm{b} * \log (\mathrm{L}))$ & -3.336 & 2.104 & 1 & 12 & (Gowing \& Recher, 1984) & not mentioned & $\begin{array}{l}\text { inferred, Hym. excl } \\
\text { Formicidae }\end{array}$ \\
\hline Isopoda & All & Dry mass & $\mathrm{M}=\exp (\mathrm{a}+\mathrm{b} * \log (\mathrm{L}))$ & -4.81 & 3.44 & 2.7 & 8 & (Wardhaugh, 2013) & $\begin{array}{l}\text { front of labrum to tip of abdomen (excl. cerci or } \\
\text { ovipositors) or tip of elytra (longest) }\end{array}$ & $\begin{array}{l}\text { Group specific, } \\
\text { Isopoda }\end{array}$ \\
\hline
\end{tabular}




\begin{tabular}{|c|c|c|c|c|c|c|c|c|c|c|}
\hline Isoptera & Rhinotermitidae & Dry mass & $\mathrm{M}=\mathrm{e}^{\wedge} \mathrm{a} * \mathrm{~L}^{\wedge} \mathrm{b}$ & -5.802 & 3.177 & 3.30 & 5.60 & (Johnson \& Strong, 2000) & head to end of abdomen & inferred, Isoptera \\
\hline Isoptera & Termitidae & Dry mass & $\mathrm{M}=\mathrm{e}^{\wedge} \mathrm{a} * \mathrm{~L}^{\wedge} \mathrm{b}$ & -5.802 & 3.177 & 3.30 & 5.60 & (Johnson \& Strong, 2000) & head to end of abdomen & inferred, Isoptera \\
\hline Isoptera & Unidentifiable & Dry mass & $\mathrm{M}=\mathrm{e}^{\wedge} \mathrm{a} * \mathrm{~L}^{\wedge} \mathrm{b}$ & -5.802 & 3.177 & 3.30 & 5.60 & (Johnson \& Strong, 2000) & head to end of abdomen & inferred, Isoptera \\
\hline Lepidoptera & Alucitidae & Dry mass & $M=\exp (a+b * \log (L))$ & -5.909 & 2.959 & 6.26 & 44.62 & (Sample, Cooper, Greer, \& Whitmore, 1993) & $\begin{array}{l}\text { frons to tip of abdomen (excl. antennae, } \\
\text { ovipositors, wings etc.) }\end{array}$ & $\begin{array}{l}\text { inferred, } \\
\text { Lepidoptera Larvae }\end{array}$ \\
\hline Lepidoptera & Arctiidae & Dry mass & $M=\exp (a+b * \log (L))$ & -5.909 & 2.959 & 6.26 & 44.62 & (Sample, Cooper, Greer, \& Whitmore, 1993) & $\begin{array}{l}\text { frons to tip of abdomen (excl. antennae, } \\
\text { ovipositors, wings etc.) }\end{array}$ & $\begin{array}{l}\text { inferred, } \\
\text { Lepidoptera Larvae }\end{array}$ \\
\hline Lepidoptera & Arctiidae & Dry mass & $M=\exp (a+b * \log (L))$ & -3.755 & 2.658 & 5.05 & 20.06 & (Sample, Cooper, Greer, \& Whitmore, 1993) & $\begin{array}{l}\text { frons to tip of abdomen (excl. antennae, } \\
\text { ovipositors, wings etc.) }\end{array}$ & $\begin{array}{l}\text { inferred, } \\
\text { Lepidoptera }\end{array}$ \\
\hline Lepidoptera & Gelechiidae & Dry mass & $M=\exp (a+b * \log (L))$ & -5.909 & 2.959 & 6.26 & 44.62 & (Sample, Cooper, Greer, \& Whitmore, 1993) & $\begin{array}{l}\text { frons to tip of abdomen (excl. antennae, } \\
\text { ovipositors, wings etc.) }\end{array}$ & $\begin{array}{l}\text { inferred, } \\
\text { Lepidoptera Larvae }\end{array}$ \\
\hline Lepidoptera & Geometridae & Dry mass & $M=\exp (a+b * \log (L))$ & -5.493 & 2.625 & 7.66 & 29.50 & (Sample, Cooper, Greer, \& Whitmore, 1993) & $\begin{array}{l}\text { frons to tip of abdomen (excl. antennae, } \\
\text { ovipositors, wings etc.) }\end{array}$ & Group specific \\
\hline Lepidoptera & Hesperiidae & Dry mass & $M=\exp (a+b * \log (L))$ & -5.909 & 2.959 & 6.26 & 44.62 & (Sample, Cooper, Greer, \& Whitmore, 1993) & $\begin{array}{l}\text { frons to tip of abdomen (excl. antennae, } \\
\text { ovipositors, wings etc.) }\end{array}$ & $\begin{array}{l}\text { inferred, } \\
\text { Lepidoptera Larvae }\end{array}$ \\
\hline Lepidoptera & Lasiocampidae & Dry mass & $M=\exp (a+b * \log (L))$ & -5.909 & 2.959 & 6.26 & 44.62 & (Sample, Cooper, Greer, \& Whitmore, 1993) & $\begin{array}{l}\text { frons to tip of abdomen (excl. antennae, } \\
\text { ovipositors, wings etc.) }\end{array}$ & $\begin{array}{l}\text { inferred, } \\
\text { Lepidoptera Larvae }\end{array}$ \\
\hline Lepidoptera & Lymantriidae & Dry mass & $M=\exp (a+b * \log (L))$ & -5.909 & 2.959 & 6.26 & 44.62 & (Sample, Cooper, Greer, \& Whitmore, 1993) & $\begin{array}{l}\text { frons to tip of abdomen (excl. antennae, } \\
\text { ovipositors, wings etc.) }\end{array}$ & $\begin{array}{l}\text { inferred, } \\
\text { Lepidoptera Larvae }\end{array}$ \\
\hline Lepidoptera & Noctuidae & Dry mass & $M=\exp (a+b * \log (L))$ & -5.424 & 2.845 & 7.96 & 42.80 & (Sample, Cooper, Greer, \& Whitmore, 1993) & $\begin{array}{l}\text { frons to tip of abdomen (excl. antennae, } \\
\text { ovipositors, wings etc.) }\end{array}$ & Group specific \\
\hline Lepidoptera & Nolidae & Dry mass & $M=\exp (a+b * \log (L))$ & -5.909 & 2.959 & 6.26 & 44.62 & (Sample, Cooper, Greer, \& Whitmore, 1993) & $\begin{array}{l}\text { frons to tip of abdomen (excl. antennae, } \\
\text { ovipositors, wings etc.) }\end{array}$ & $\begin{array}{l}\text { inferred, } \\
\text { Lepidoptera Larvae }\end{array}$ \\
\hline Lepidoptera & Pterophoridae & Dry mass & $M=\exp (a+b * \log (L))$ & -5.909 & 2.959 & 6.26 & 44.62 & (Sample, Cooper, Greer, \& Whitmore, 1993) & $\begin{array}{l}\text { frons to tip of abdomen (excl. antennae, } \\
\text { ovipositors, wings etc.) }\end{array}$ & $\begin{array}{l}\text { inferred, } \\
\text { Lepidoptera Larvae }\end{array}$ \\
\hline Lepidoptera & Pyralidae & Dry mass & $M=\exp (a+b * \log (L))$ & -5.909 & 2.959 & 6.26 & 44.62 & (Sample, Cooper, Greer, \& Whitmore, 1993) & $\begin{array}{l}\text { frons to tip of abdomen (excl. antennae, } \\
\text { ovipositors, wings etc.) }\end{array}$ & $\begin{array}{l}\text { inferred, } \\
\text { Lepidoptera Larvae }\end{array}$ \\
\hline Lepidoptera & Pyralidae & Dry mass & $M=\exp (a+b * \log (L))$ & -5.036 & 3.122 & 2.76 & 40.73 & (Sample, Cooper, Greer, \& Whitmore, 1993) & $\begin{array}{l}\text { frons to tip of abdomen (excl. antennae, } \\
\text { ovipositors, wings etc.) }\end{array}$ & $\begin{array}{l}\text { inferred, } \\
\text { Lepidoptera }\end{array}$ \\
\hline Mantodea & Mantidae & Dry mass & $M=\exp (a+b * \log (L))$ & -6.340 & 3.010 & 6.00 & 66.00 & (Wardhaugh, 2013) & $\begin{array}{l}\text { front of labrum to tip of abdomen (excl. cerci or } \\
\text { ovipositors) or tip of elytra (longest) }\end{array}$ & Group specific \\
\hline Neuroptera & Chrysopidae & Dry mass & $M=\exp (a+b * \log (L))$ & -4.483 & 2.570 & 3.45 & 54.51 & (Sample, Cooper, Greer, \& Whitmore, 1993) & $\begin{array}{l}\text { frons to tip of abdomen (excl. antennae, } \\
\text { ovipositors, wings etc.) }\end{array}$ & $\begin{array}{l}\text { inferred, } \\
\text { Neuroptera }\end{array}$ \\
\hline Opiliones & All & Fresh mass & $M=\exp (a+b * \log (L))$ & -0.899 & 2.984 & 0.57 & 6.9 & (Höfer \& Ott, 2009) & $\begin{array}{l}\text { edge of prosoma (without chelicerae) to edge of } \\
\text { opisthosoma (excl spinnerets) }\end{array}$ & inferred, Opiliones \\
\hline Orthoptera & Acrididae & Dry mass & $M=\exp (a+b * \log (L))$ & -3.17 & 2.61 & 2.3 & 33 & (Wardhaugh, 2013) & $\begin{array}{l}\text { front of labrum to tip of abdomen (excl. cerci or } \\
\text { ovipositors) or tip of elytra (longest) }\end{array}$ & inferred, Orthoptera \\
\hline Orthoptera & Eumastacidae & Dry mass & $M=\exp (a+b * \log (L))$ & -3.17 & 2.61 & 2.3 & 33 & (Wardhaugh, 2013) & $\begin{array}{l}\text { front of labrum to tip of abdomen (excl. cerci or } \\
\text { ovipositors) or tip of elytra (longest) }\end{array}$ & inferred, Orthoptera \\
\hline
\end{tabular}




\begin{tabular}{|c|c|c|c|c|c|c|c|c|c|c|}
\hline Orthoptera & Gryllidae & Dry mass & $M=\exp (a+b * \log (L))$ & -3.17 & 2.61 & 2.3 & 33 & (Wardhaugh, 2013) & $\begin{array}{l}\text { front of labrum to tip of abdomen (excl. cerci or } \\
\text { ovipositors) or tip of elytra (longest) }\end{array}$ & inferred, Orthoptera \\
\hline Orthoptera & Tetrigidae & Dry mass & $M=\exp (a+b * \log (L))$ & -3.17 & 2.61 & 2.3 & 33 & (Wardhaugh, 2013) & $\begin{array}{l}\text { front of labrum to tip of abdomen (excl. cerci or } \\
\text { ovipositors) or tip of elytra (longest) }\end{array}$ & inferred, Orthoptera \\
\hline Plecoptera & All & Dry mass & $M=a * L^{\wedge} b$ & 0.0094 & 2.754 & 1.95 & 3.232 & (Benke, Huryn, Smock, \& Wallace, 1999) & Total length & Group specific \\
\hline Plecoptera & Austroperlidae & Dry mass & $\mathrm{M}=\mathrm{a} * \mathrm{~L}^{\wedge} \mathrm{b}$ & 0.0094 & 2.754 & 1.95 & 3.232 & (Benke, Huryn, Smock, \& Wallace, 1999) & Total length & Group specific \\
\hline Plecoptera & Gripopterygidae & Dry mass & $M=a * L^{\wedge} b$ & 0.0094 & 2.754 & 1.95 & 3.232 & (Benke, Huryn, Smock, \& Wallace, 1999) & Total length & Group specific \\
\hline Plecoptera & Notonemouridae & Dry mass & $M=a * L^{\wedge} b$ & 0.0094 & 2.754 & 1.95 & 3.232 & (Benke, Huryn, Smock, \& Wallace, 1999) & Total length & Group specific \\
\hline Pseudoscorpionida & All & fresh mass & $\mathrm{M}=\exp (\mathrm{a}+\mathrm{b} * \log (\mathrm{L}))$ & -1.892 & 2.515 & 0.86 & 2.10 & (Höfer \& Ott, 2009) & $\begin{array}{l}\text { edge of prosoma (without chelicerae) to edge of } \\
\text { opisthosoma (excl spinnerets) }\end{array}$ & Group specific \\
\hline Psocoptera & Archipsocidae & Dry mass & $M=a *(L)^{\wedge} b$ & 0.014 & 3.115 & 1.50 & 3.15 & (Gruner, 2003) & $\begin{array}{l}\text { tip of abdomen to end of head or carapace, excl. } \\
\text { any appendages }\end{array}$ & inferred, Psocoptera \\
\hline Psocoptera & Caeciliidae & Dry mass & $\mathrm{M}=\mathrm{a} *(\mathrm{~L})^{\wedge} \mathrm{b}$ & 0.014 & 3.115 & 1.50 & 3.15 & (Gruner, 2003) & $\begin{array}{l}\text { tip of abdomen to end of head or carapace, excl. } \\
\text { any appendages }\end{array}$ & inferred, Psocoptera \\
\hline Psocoptera & Ectopsocidae & Dry mass & $\mathrm{M}=\mathrm{a} *(\mathrm{~L})^{\wedge} \mathrm{b}$ & 0.014 & 3.115 & 1.50 & 3.15 & (Gruner, 2003) & $\begin{array}{l}\text { tip of abdomen to end of head or carapace, excl. } \\
\text { any appendages }\end{array}$ & inferred, Psocoptera \\
\hline Psocoptera & Elipsocidae & Dry mass & $M=a *(L)^{\wedge} b$ & 0.014 & 3.115 & 1.50 & 3.15 & (Gruner, 2003) & $\begin{array}{l}\text { tip of abdomen to end of head or carapace, excl. } \\
\text { any appendages }\end{array}$ & inferred, Psocoptera \\
\hline Psocoptera & Epipsocidae & Dry mass & $\mathrm{M}=\mathrm{a} *(\mathrm{~L})^{\wedge} \mathrm{b}$ & 0.014 & 3.115 & 1.50 & 3.15 & (Gruner, 2003) & $\begin{array}{l}\text { tip of abdomen to end of head or carapace, excl. } \\
\text { any appendages }\end{array}$ & inferred, Psocoptera \\
\hline Psocoptera & Hemipsocidae & Dry mass & $M=a *(L)^{\wedge} b$ & 0.014 & 3.115 & 1.50 & 3.15 & (Gruner, 2003) & $\begin{array}{l}\text { tip of abdomen to end of head or carapace, excl. } \\
\text { any appendages }\end{array}$ & inferred, Psocoptera \\
\hline Psocoptera & Lepidopsocidae & Dry mass & $M=a *(L)^{\wedge} b$ & 0.014 & 3.115 & 1.50 & 3.15 & (Gruner, 2003) & $\begin{array}{l}\text { tip of abdomen to end of head or carapace, excl. } \\
\text { any appendages }\end{array}$ & inferred, Psocoptera \\
\hline Psocoptera & Pachytroctidae & Dry mass & $M=a *(L)^{\wedge} b$ & 0.014 & 3.115 & 1.50 & 3.15 & (Gruner, 2003) & $\begin{array}{l}\text { tip of abdomen to end of head or carapace, excl. } \\
\text { any appendages }\end{array}$ & inferred, Psocoptera \\
\hline Psocoptera & Psocidae & Dry mass & $M=a *(L)^{\wedge} b$ & 0.014 & 3.115 & 1.50 & 3.15 & (Gruner, 2003) & $\begin{array}{l}\text { tip of abdomen to end of head or carapace, excl. } \\
\text { any appendages }\end{array}$ & inferred, Psocoptera \\
\hline Psocoptera & Psyllipsocidae & Dry mass & $M=a *(L)^{\wedge} b$ & 0.014 & 3.115 & 1.50 & 3.15 & (Gruner, 2003) & $\begin{array}{l}\text { tip of abdomen to end of head or carapace, excl. } \\
\text { any appendages }\end{array}$ & inferred, Psocoptera \\
\hline Psocoptera & Unidentifiable & Dry mass & $\mathrm{M}=\mathrm{a} *(\mathrm{~L})^{\wedge} \mathrm{b}$ & 0.014 & 3.115 & 1.50 & 3.15 & (Gruner, 2003) & $\begin{array}{l}\text { tip of abdomen to end of head or carapace, excl. } \\
\text { any appendages }\end{array}$ & inferred, Psocoptera \\
\hline Schizomida & Hubbardiidae & Fresh mass & $M=\exp (a+b * \log (L))$ & -2.108 & 3.017 & 0.67 & 36 & (Höfer \& Ott, 2009) & $\begin{array}{l}\text { edge of prosoma (without chelicerae) to edge of } \\
\text { opisthosoma (excl spinnerets) }\end{array}$ & $\begin{array}{l}\text { inferred, hunting } \\
\text { spiders }\end{array}$ \\
\hline Symphyla & Scutegerillidae & Dry mass & $\mathrm{M}=\mathrm{a} *(\mathrm{~L})^{\wedge} \mathrm{b}$ & 0.035 & 2.173 & 0.9 & 17.6 & (Gruner, 2003) & $\begin{array}{l}\text { tip of abdomen to end of head or carapace, excl. } \\
\text { any appendages }\end{array}$ & $\begin{array}{l}\text { inferred, all insect } \\
\text { taxa }\end{array}$ \\
\hline Thysanoptera & Aeolothripidae & Dry mass & $\mathrm{M}=\mathrm{a} *(\mathrm{~L})^{\wedge} \mathrm{b}$ & 0.035 & 2.173 & 0.9 & 17.6 & (Gruner, 2003) & $\begin{array}{l}\text { tip of abdomen to end of head or carapace, excl. } \\
\text { any appendages }\end{array}$ & $\begin{array}{l}\text { inferred, all insect } \\
\text { taxa }\end{array}$ \\
\hline
\end{tabular}




\begin{tabular}{|c|c|c|c|c|c|c|c|c|c|c|}
\hline Thysanoptera & Phlaeothripidae & Dry mass & $\mathrm{M}=\mathrm{a} *(\mathrm{~L})^{\wedge} \mathrm{b}$ & 0.035 & 2.173 & 0.9 & 17.6 & (Gruner, 2003) & $\begin{array}{l}\text { tip of abdomen to end of head or carapace, excl. } \\
\text { any appendages }\end{array}$ & $\begin{array}{l}\text { inferred, all insect } \\
\text { taxa }\end{array}$ \\
\hline Thysanoptera & Thripidae & Dry mass & $M=a *(L)^{\wedge} b$ & 0.035 & 2.173 & 0.9 & 17.6 & (Gruner, 2003) & $\begin{array}{l}\text { tip of abdomen to end of head or carapace, excl. } \\
\text { any appendages }\end{array}$ & $\begin{array}{l}\text { inferred, all insect } \\
\text { taxa }\end{array}$ \\
\hline Thysanura & Nicoletiidae & Dry mass & $M=\exp (a+b * \log (L))$ & -3.628 & 2.494 & 2.13 & 54.51 & (Sample, Cooper, Greer, \& Whitmore, 1993) & $\begin{array}{l}\text { From frons to tip of abdomen excluding } \\
\text { appendages }\end{array}$ & $\begin{array}{l}\text { inferred, all insect } \\
\text { taxa }\end{array}$ \\
\hline
\end{tabular}

Supplementary Table 3.1b Dry-to-fresh mass conversions from the literature ${ }^{12}$ for transformation of dry body masses (DM) (from length-dry mass regression calculations) to fresh mass (FM).

\begin{tabular}{lllll}
\hline Taxon & Equation FM[mg], DM[mg] & a & b & Reference \\
\hline Annelida & $\mathrm{FM}=\exp (\mathrm{a}+\mathrm{b} * \log (\mathrm{DM}))$ & 0.9282 & 1.0899 (Mercer et al., 2001$)$ & Oligochaeta \\
$\begin{array}{l}\text { All other groups with dry-mass length-mass regressions, } \\
\text { (see Supplementary Table S4a) }\end{array}$ & $\mathrm{FM}=\exp (\mathrm{a}+\mathrm{b} * \log (\mathrm{DM}))$ & 0.6111 & 1.0213 (Mercer et al., 2001) \\
\hline
\end{tabular}


Supplementary Table 3.2 Regression parameters for individual metabolic rate calculation from the literature ${ }^{13}$ and unpublished data (Roswitha Ehnes). Phylogenetic model: $\ln \mathrm{I}=\ln \mathrm{i}_{\mathrm{oPG}}+\mathrm{a}_{\mathrm{PG}} \ln$ $\mathrm{M}-\mathrm{E}_{\mathrm{PG}}(1 / \mathrm{kT}) ;$ Linear model: $\ln \mathrm{I}=\ln \mathrm{i}_{\mathrm{o}}+\mathrm{a} \ln \mathrm{M}-\mathrm{E}(1 / \mathrm{kT})$. $\mathrm{I}$ is the metabolic rate, $\mathrm{a}$ is the allometric exponent, $\mathrm{E}$ is the activation energy, $\mathrm{k}$ is the Boltzmann constant, $\mathrm{T}$ the temperature in Kelvin (in our models taken as local mean soil temperature) and $i_{o}$ a normalisation factor.

\begin{tabular}{|c|c|c|c|c|c|}
\hline Regression group & Applied to taxa & $\begin{array}{c}\ln \mathbf{i}_{\mathbf{o}} \\
/ \ln \mathbf{i}_{\mathrm{oPG}}\end{array}$ & $\begin{array}{c}\mathbf{a} \\
\mathbf{a}_{\mathbf{P G}}\end{array}$ & $\begin{array}{c}\mathbf{E} \\
\mathbf{E}_{\mathbf{P G}}\end{array}$ & Model \\
\hline Arachnida & $\begin{array}{l}\text { Araneae, Opiliones, } \\
\text { Pseudoscorpionida, } \\
\text { Schizomida }\end{array}$ & 24.581 & 0.565 & 0.709 & phylogenetic \\
\hline Chilopoda & Chilopoda & 28.253 & 0.558 & 0.803 & phylogenetic \\
\hline Clitellata & Clitellata & 12.442 & 0.801 & 0.443 & phylogenetic \\
\hline Coleoptera & Coleoptera & 21.418 & 0.738 & 0.639 & phylogenetic \\
\hline General invertebrates & Gastropoda & 23.055 & 0.695 & 0.686 & linear \\
\hline Hymenoptera & Hymenoptera & 22.013 & 0.742 & 0.668 & phylogenetic \\
\hline Insecta & $\begin{array}{l}\text { Arachaeognatha, Blattodea, } \\
\text { Dermaptera, Diplura, Diptera, } \\
\text { Hemiptera, Isoptera, } \\
\text { Lepidoptera, Mantodea, } \\
\text { Neuroptera, Orthoptera, } \\
\text { Plecoptera, Psocoptera, } \\
\text { Symphyla, Thysanoptera } \\
\text { Thysanura }\end{array}$ & 21.972 & 0.759 & 0.657 & phylogenetic \\
\hline Isopoda & Isopoda & 23.169 & 0.554 & 0.687 & phylogenetic \\
\hline Progoneata & Diplopoda & 22.347 & 0.571 & 0.670 & phylogenetic \\
\hline
\end{tabular}


Supplementary Table 3.3 Summary and ANOVA tables from the best-fit generalised linear mixed effects models as selected by AIC: (a) negative binomial model testing the effects of transformation system (TrSys) and functional feeding guild (FFG) on species richness (SpRichness); (b) gaussian models testing the effects of transformation system (TrSys) and functional feeding guild (FFG) on density, biomass, and community metabolism (CM). Asterisks denote significance levels: $* \mathrm{p}<0.05 ; * * \mathrm{p}<0.01 ; * * * \mathrm{p}<0.001$.

(a)

\begin{tabular}{llcrrrr}
\hline Model & Fixed effects & Estimate & Std. Error & z value & Pr $(>|\mathbf{z}|)$ & \\
\hline SpRichness & Intercept & 3.088 & 0.127 & 24.290 & 0.000 & $* * *$ \\
TrSys * FFG & Jungle rubber & 0.054 & 0.179 & 0.299 & 0.766 & \\
& Oil palm & -0.556 & 0.190 & -2.921 & 0.003 & $* *$ \\
& Rubber & -0.275 & 0.184 & -1.491 & 0.135 & \\
& Omnivores & 0.168 & 0.101 & 1.668 & 0.096 & \\
& Herbivores & -1.759 & 0.194 & -9.065 & 0.000 & $* * *$ \\
& Predators & 0.606 & 0.092 & 6.553 & 0.000 & $* * *$ \\
& Jungle rubber : Omnivores & -0.399 & 0.149 & -2.685 & 0.007 & $* * *$ \\
& Oil palm : Omnivores & -0.087 & 0.168 & -0.520 & 0.603 & \\
& Rubber : Omnivores & -0.099 & 0.154 & -0.644 & 0.519 & \\
& Jungle rubber : Herbivores & 0.346 & 0.254 & 1.361 & 0.174 & \\
& Oil palm : Herbivores & 0.641 & 0.274 & 2.337 & 0.019 & $*$ \\
& Rubber : Herbivores & 0.953 & 0.246 & 3.876 & 0.000 & $* * *$ \\
& Jungle rubber : Predators & -0.253 & 0.132 & -1.912 & 0.056 & \\
& Oil palm : Predators & -0.235 & 0.156 & -1.510 & 0.131 & \\
& Rubber : Predators & -0.078 & 0.141 & -0.556 & 0.578 & \\
\hline
\end{tabular}

(b)

\begin{tabular}{llcrrrr}
\hline Model & Fixed effects & numDF & denDF & F-value & $\operatorname{Pr}(>|\mathbf{z}|)$ \\
\hline Density $\sim$ TrSys * FFG & TrSys & 3 & 27 & 0.363 & 0.780 & \\
& FFG & 3 & 84 & 77.611 & 0.000 & $* * *$ \\
& TrSys : FFG & 9 & 84 & 3.432 & 0.001 & $* *$ \\
& & & & & & \\
Biomass $\sim$ TrSys + FFG & TrSys & 3 & 27 & 3.570 & 0.027 & $*$ \\
& FFG & 3 & 93 & 38.759 & 0.000 & $* * *$ \\
& & & & & & \\
CM $\sim$ TrSys + FFG & TrSys & 3 & 27 & 3.456 & 0.030 & $*$ \\
& FFG & 3 & 93 & 64.825 & 0.000 & $* * *$ \\
\hline
\end{tabular}


Supplementary Table 3.4 Energy flux and fresh biomass values for the four functional feeding guilds (FFG) and four transformation systems. Energy flux is expressed as $\mathrm{kg}$ fresh mass $\left[\mathrm{ha}^{-1} \mathrm{yr}^{-}\right.$ ${ }^{1}$ ] using a conversion factor ${ }^{1}: 1 \mathrm{~kg}$ wet mass $=7 * 10^{6} \mathrm{~J}$.

\begin{tabular}{llcc}
\hline FFG & Transformation system & $\begin{array}{c}\text { Energy flux } \\
{\left[\mathbf{k g ~ h a}^{-\mathbf{1}} \mathbf{\mathbf { y r } ^ { - \mathbf { 1 } } ]}\right.}\end{array}$ & $\begin{array}{c}\text { Biomass } \\
{\left[\mathbf{k g ~ h a}^{-\mathbf{1}}\right]}\end{array}$ \\
\hline Omnivore & Forest & 61.900 & 0.629 \\
Omnivore & Jungle rubber & 52.313 & 0.494 \\
Omnivore & Rubber & 55.880 & 0.751 \\
Omnivore & Oil palm & 32.531 & 0.766 \\
Detritivore & Forest & 200.187 & 1.039 \\
Detritivore & Jungle rubber & 160.165 & 0.558 \\
Detritivore & Rubber & 164.194 & 0.504 \\
Detritivore & Oil palm & 94.440 & 0.352 \\
Predator & Forest & 66.816 & 1.664 \\
Predator & Jungle rubber & 53.248 & 0.976 \\
Predator & Rubber & 55.454 & 0.954 \\
Predator & Oil palm & 30.697 & 0.424 \\
Herbivore & Forest & 87.537 & 0.093 \\
Herbivore & Jungle & 75.389 & 0.139 \\
Herbivore & Rubber & 83.288 & 0.319 \\
Herbivore & Oil palm & 44.316 & 0.076 \\
\hline
\end{tabular}


Supplementary Table 3.5 ANOVA tables from the generalised linear mixed effects models testing the effects of transformation system (TrSys), species richness (SpRichness), and their interaction on energy flux (EF) for the total community data set and also separated into functional feeding guilds (FFG). All models displayed are those that were selected as the best-fit model from the stepwise AIC selection procedure. Asterisks denote significance levels: $* \mathrm{p}<0.05 ; * * \mathrm{p}<$ $0.01 ; * * * \mathrm{p}<0.001$.

\begin{tabular}{llccccc}
\hline Model & Fixed effects & numDF & denDF & F-value & $\operatorname{Pr}(>|\mathbf{z}|)$ \\
\hline Total Community & TrSys & 3 & 23 & 5.226 & 0.007 & $* *$ \\
EF $\sim$ TrSys * SpRichness & SpRichness & 1 & 23 & 4.965 & 0.036 & $*$ \\
& TrSys : SpRichness & 3 & 23 & 4.637 & 0.011 & $*$
\end{tabular}

\section{Omnivores}

$\mathrm{EF} \sim$ SpRichness

SpRichness

$\begin{array}{llll}1 & 29 & 42.842 & 0.000\end{array}$

\section{Detritivores}

$\mathrm{EF} \sim$ TrSys + SpRichness

TrSys

SpRichness

$\begin{array}{rrrrl}3 & 26 & 3.103 & 0.044 & * \\ 1 & 26 & 22.285 & 0.000 & * * *\end{array}$

Predators

$\mathrm{EF} \sim \mathrm{TrSys} *$ SpRichness

TrSys

SpRichness

TrSys : SpRichness

$\begin{array}{lllll}3 & 23 & 5.507 & 0.005 & * * \\ 1 & 23 & 5.813 & 0.024 & * \\ 3 & 23 & 4.618 & 0.011 & *\end{array}$

Herbivores

TrSys

$\begin{array}{lllll}3 & 26 & 5.944 & 0.003 & * * \\ 1 & 26 & 9.436 & 0.005 & * *\end{array}$

$\mathrm{EF} \sim$ TrSys + SpRichness

SpRichness 
Supplementary Methods 3.1 Calculation of energy fluxes $(F)$ from community metabolism $(X)$, assimilation efficiencies (e), and losses to predation $(L) . O, P, D, H, P l$ and $D t$ denote omnivores, predators, detritivores, herbivores, plants and detritus. We denote total flux to a node $I$ as $F_{I}$ and the flux from node $J$ to $I$ as $F_{I J}$. For example, $F_{O}$ is the total flux to omnivores and $F_{O P}$ is the flux from predators to omnivores. Assimilation efficiencies of animal food (0.60), plant food (0.45) and detritus food $(0.25)^{14}$ are given as $e_{a}, e_{p}$, and $e_{d}$, respectively.

$$
F_{O}=F_{O P}+F_{O H}+F_{O D}+F_{O P l}+F_{O D t}
$$

We assume that predators, herbivores and detritivores each contribute to $1 / 4$ of the omnivore diet and plants and detritus equally contribute to the remaining $1 / 4$.

$$
\begin{gathered}
F_{O P}=F_{O H}=F_{O D}=\frac{1}{4} F_{O} \\
F_{O P l}=F_{O D t}=\frac{1}{8} F_{O}
\end{gathered}
$$

The community metabolism $X$ of a node is given as

$$
X=(F \cdot e)-L
$$

Thus, the energy entering the omnivore node is given as

$$
X_{O}+L=e_{a} \cdot\left(F_{O P}+F_{O H}+F_{O D}\right)+e_{p} \cdot F_{O P l}+e_{d} \cdot F_{O D t}=\left(\frac{3}{4} e_{a}+\frac{1}{8} e_{p}+\frac{1}{8} e_{d}\right) \cdot F_{O},
$$

where equations 2 and 3 were used to replace single fluxes with the fraction of the overall flux.

The efficiency with which omnivores assimilate resources is

$$
e_{O}=\left(\frac{3}{4} e_{a}+\frac{1}{8} e_{p}+\frac{1}{8} e_{d}\right)
$$

Now, to express $F_{O}, e_{O}$ needs to be replaced by equation 6 , which yields

$$
F_{O}=\frac{1}{e_{O}} \cdot\left(X_{O}+\frac{F_{P}}{3}\right)
$$

The equation for predators is similar but with the $e_{a}$ assimilation efficiency, yielding

$$
F_{P}=\frac{1}{e_{a}} \cdot\left(X_{P}+\frac{F_{O}}{4}\right)
$$

We then solve for $F_{P}$ by inserting equation 7 into 8 : 


$$
F_{P}=\frac{12 \cdot e_{O} \cdot X_{P}+3 \cdot X_{O}}{12 \cdot e_{a} \cdot e_{O}-1}
$$

Now we calculate $F_{O}$ using equation 7 , and, with $F_{P}$ and $F_{O}$ we can calculate $F_{H}$ and $F_{D}$ using equations

$$
F_{H}=\frac{1}{e_{p}} \cdot\left(X_{H}+\frac{F_{P}}{3}+\frac{F_{O}}{4}\right)
$$

and

$$
F_{D}=\frac{1}{e_{d}} \cdot\left(X_{D}+\frac{F_{P}}{3}+\frac{F_{O}}{4}\right)
$$




\section{SUPPLEMENTARY INFORMATION - CHAPTER 4}

Supplementary Table 4.1 Functional response and effect groups from sampled macro-invertebrate communities. Traits and species richness within the eleven functional response groups and the six functional effect groups. Dispersal capacity is used as a response trait only. LW: legs and wings, LN: legs but no wings, NN: no legs, no wings.

\begin{tabular}{|c|c|c|c|c|c|c|c|}
\hline \multirow{2}{*}{ Response group } & \multirow{2}{*}{ Feeding type } & \multirow{2}{*}{ Eusociality } & \multirow{2}{*}{$\begin{array}{c}\text { Dispersal } \\
\text { capacity }\end{array}$} & \multicolumn{2}{|c|}{ Body mass (mg) } & \multirow{2}{*}{ Species richness } & \multirow{2}{*}{ Abundance } \\
\hline & & & & Mean & Standard error & & \\
\hline 1 & Detritivore & no & $\mathrm{NN}$ & 21.079 & \pm 8.679 & 6 & 58 \\
\hline 2 & Predator & no & $\mathrm{LN}$ & 2.816 & \pm 0.157 & 278 & 1245 \\
\hline 3 & Predator & no & $\mathrm{LN}$ & 53.352 & \pm 7.695 & 12 & 90 \\
\hline 4 & Omnivore & no & $\mathrm{LN}$ & 6.027 & \pm 1.379 & 28 & 157 \\
\hline 5 & Detritivore & no & LW & 2.089 & \pm 0.147 & 78 & 581 \\
\hline 6 & Detritivore & no & LW & 84.351 & \pm 22.702 & 6 & 17 \\
\hline 7 & Detritivore & no & LW & 1.199 & \pm 0.173 & 85 & 803 \\
\hline 8 & Herbivore & no & LW & 4.055 & \pm 0.457 & 86 & 251 \\
\hline 9 & Predator & no & LW & 1.462 & \pm 0.306 & 139 & 430 \\
\hline 10 & Fungivore & no & LW & 0.886 & \pm 0.112 & 24 & 73 \\
\hline 11 & Omnivore & no & LW & 8.880 & \pm 5.238 & 21 & 60 \\
\hline 12 & Parasite & no & $\mathrm{LN}$ & 1.630 & \pm 1.549 & 4 & 4 \\
\hline 13 & Parasite & no & LW & 2.570 & \pm 2.046 & 2 & 2 \\
\hline 14 & Herbivore & no & $\mathrm{LN}$ & 23.221 & \pm 23.171 & 2 & 2 \\
\hline 15 & Herbivore & no & LW & 224.699 & \pm 75.026 & 2 & 2 \\
\hline 16 & Omnivore & yes & LW & 0.983 & \pm 0.048 & 98 & 2963 \\
\hline 17 & Detritivore & yes & LW & 0.342 & \pm 0.014 & 14 & 778 \\
\hline 18 & Omnivore & no & $\mathrm{NN}$ & 26.186 & \pm 5.075 & 11 & 28 \\
\hline
\end{tabular}




\begin{tabular}{|c|c|c|c|c|c|c|}
\hline \multicolumn{7}{|c|}{ Effect group } \\
\hline 1 & Detritivore & no & 1.242 & \pm 0.081 & 157 & 1411 \\
\hline 2 & Detritivore & no & 109.880 & \pm 22.468 & 6 & 22 \\
\hline 3 & Predator & no & 1.400 & \pm 0.063 & 375 & 1524 \\
\hline 4 & Predator & no & 12.061 & \pm 0.904 & 39 & 147 \\
\hline 5 & Predator & no & 53.298 & \pm 7.389 & 15 & 94 \\
\hline 6 & Omnivore & no & 5.886 & \pm 0.638 & 56 & 239 \\
\hline 7 & Detritivore & no & 23.246 & \pm 2.717 & 12 & 26 \\
\hline 8 & Herbivore & no & 4.207 & \pm 0.484 & 88 & 253 \\
\hline 9 & Fungivore & no & 0.886 & \pm 0.112 & 24 & 73 \\
\hline 10 & Parasite & no & 1.943 & \pm 1.130 & 6 & 6 \\
\hline 11 & Herbivore & no & 224.699 & \pm 75.026 & 2 & 2 \\
\hline 12 & Omnivore & yes & 0.983 & \pm 0.048 & 98 & 2963 \\
\hline 13 & Detritivore & yes & 0.342 & \pm 0.014 & 14 & 778 \\
\hline 14 & Omnivore & no & 134.228 & \pm 41.966 & 4 & 6 \\
\hline
\end{tabular}


Supplementary Figure 4.1 Estimated species richness and sample coverage of sampled macroinvertebrates. Sample-based species accumulation curves show the relative differences in sampled species richness among the four different land-use types; forest, jungle rubber, rubber monoculture, and oil palm. Sample coverage shows the mean proportion of observed species richness out of the total (extrapolated) species richness, using the second order jacknife estimator.
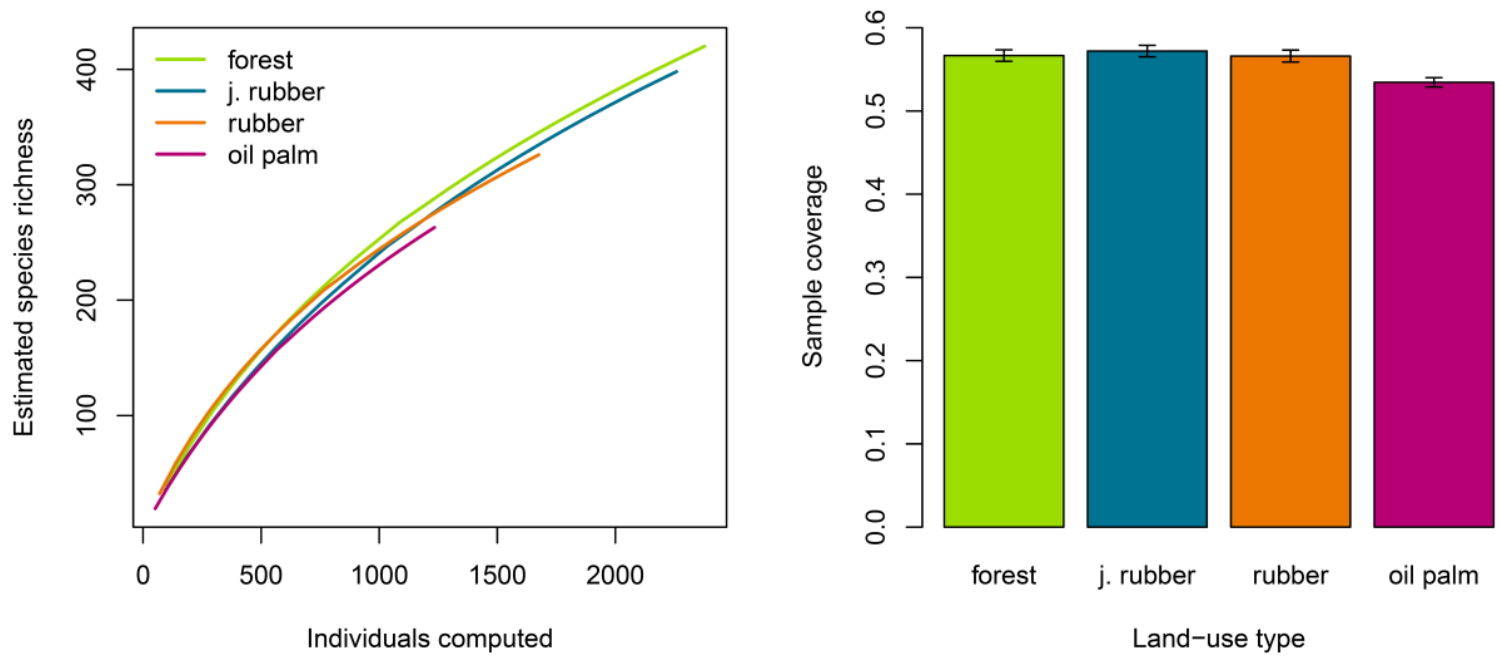
Supplementary Table 4.2 Total abundance and species richness (i.e., number of morphospecies, in brackets) of taxonomic groups across the four land-use systems. Presented taxonomic groups are mostly at the order level, but with some at the class level.

\begin{tabular}{|c|c|c|c|c|c|}
\hline \multirow{2}{*}{ Taxonomic group } & \multicolumn{4}{|c|}{ Land-use system } & \multirow{2}{*}{ Total } \\
\hline & Forest & Jungle rubber & Rubber & Oil palm & \\
\hline Hymenoptera & $1044(67)$ & $1058(58)$ & $498(50)$ & $386(45)$ & $2986(112)$ \\
\hline Araneae & $332(102)$ & $307(82)$ & $266(78)$ & $173(86)$ & $1078(251)$ \\
\hline Isoptera & $301(7)$ & $107(9)$ & $29(6)$ & $341(6)$ & $778(14)$ \\
\hline Psocoptera & $13(5)$ & $161(11)$ & $266(12)$ & $67(12)$ & $507(17)$ \\
\hline Coleoptera & $196(71)$ & $153(76)$ & $103(52)$ & $28(19)$ & $480(167)$ \\
\hline Blattodea & $78(21)$ & $106(17)$ & $109(17)$ & $33(8)$ & $326(25)$ \\
\hline Lepidoptera & $20(13)$ & $49(21)$ & $74(21)$ & $20(17)$ & $163(41)$ \\
\hline Diplopoda & $32(16)$ & $72(16)$ & $28(5)$ & $17(5)$ & $149(26)$ \\
\hline Hemiptera & $37(24)$ & $47(30)$ & $46(25)$ & $19(11)$ & $149(74)$ \\
\hline Chilopoda & $45(8)$ & $22(8)$ & $44(7)$ & $25(9)$ & $136(15)$ \\
\hline Diptera & $17(8)$ & $21(12)$ & $93(14)$ & $3(3)$ & $134(26)$ \\
\hline Isopoda & $68(22)$ & $42(16)$ & $4(3)$ & $14(8)$ & $128(36)$ \\
\hline Orthoptera & $37(14)$ & $36(11)$ & $39(15)$ & $12(7)$ & $124(23)$ \\
\hline Opiliones & $36(10)$ & $22(8)$ & $1(1)$ & $4(2)$ & $63(14)$ \\
\hline Annelida & $23(5)$ & $5(2)$ & $12(2)$ & $18(4)$ & $58(6)$ \\
\hline Plecoptera & $28(6)$ & $8(2)$ & $4(2)$ & $6(2)$ & $46(6)$ \\
\hline Pseudoscorpionida & $39(5)$ & $5(2)$ & $1(1)$ & $1(1)$ & $46(5)$ \\
\hline Thysanoptera & $1(1)$ & $13(5)$ & $17(4)$ & $10(5)$ & $41(9)$ \\
\hline Symphyla & $4(2)$ & $0(0)$ & $4(1)$ & $27(1)$ & $35(2)$ \\
\hline Dermaptera & $4(3)$ & $1(1)$ & $12(3)$ & $17(5)$ & $34(5)$ \\
\hline Gastropoda & $4(4)$ & $4(2)$ & $15(2)$ & $5(3)$ & $28(11)$ \\
\hline Archaeognatha & $12(3)$ & $8(1)$ & $0(0)$ & $0(0)$ & $20(3)$ \\
\hline Mantodea & $0(0)$ & $6(3)$ & $6(3)$ & $3(1)$ & $15(4)$ \\
\hline Diplura & $5(1)$ & $2(1)$ & $3(1)$ & $3(1)$ & $13(1)$ \\
\hline Neuroptera & $0(0)$ & $2(1)$ & $2(1)$ & $0(0)$ & $4(1)$ \\
\hline Schizomida & $0(0)$ & $1(1)$ & $0(0)$ & $1(1)$ & $2(1)$ \\
\hline Thysanura & $0(0)$ & $1(1)$ & $0(0)$ & $0(0)$ & $1(1)$ \\
\hline
\end{tabular}


Supplementary Figure 4.2 Density of the 18 functional response groups (FG) (see

Supplementary Table 4.1 for descriptions of functional response groups) in the four land-use systems: forest $(\mathrm{F})$, jungle rubber $(\mathrm{J})$, rubber $(\mathrm{R})$ and oil palm $(\mathrm{O})$.

a)

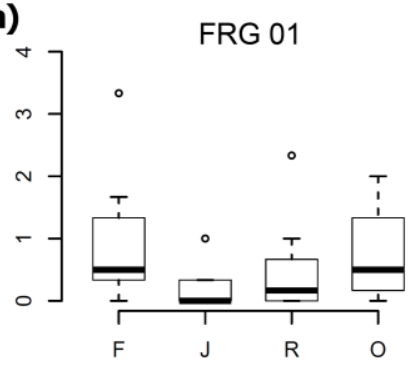

$\overline{\mathrm{N}}_{\mathrm{E}}$ d)

FRG 04
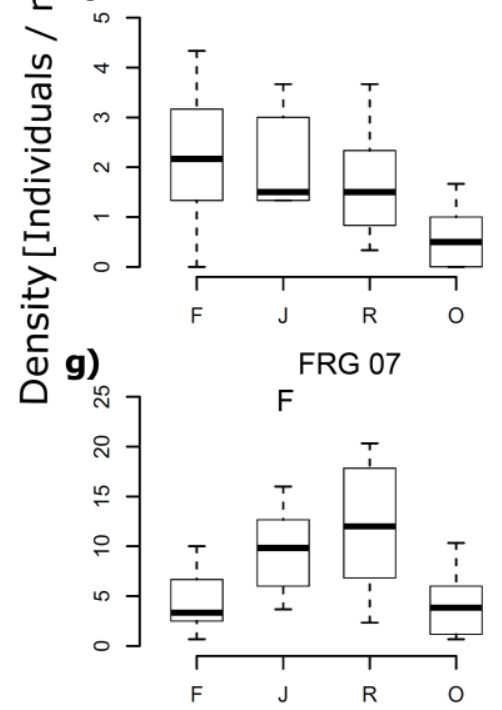

b)

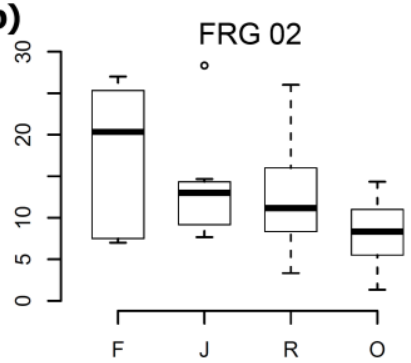

e)

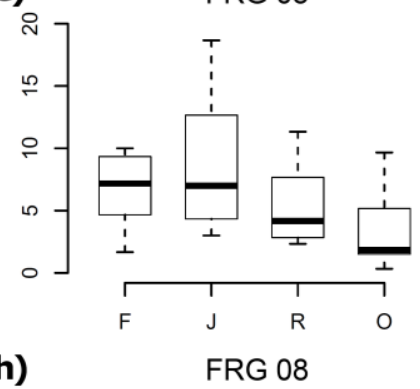

h)

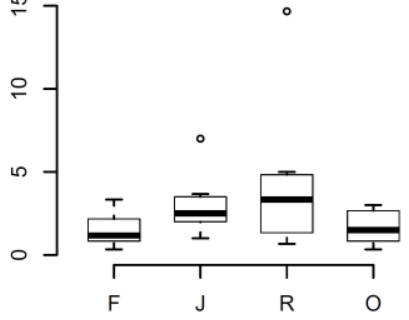

c)

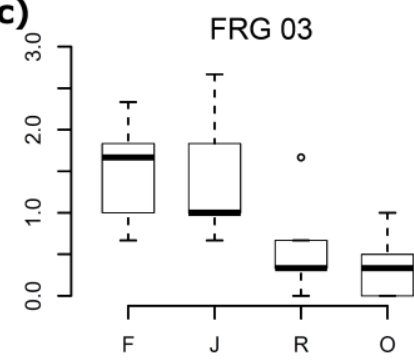

f)

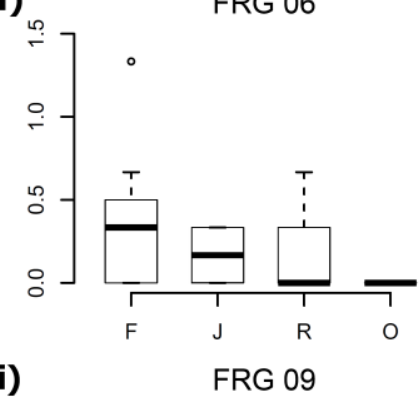

i)

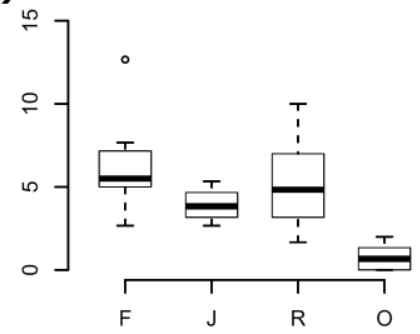



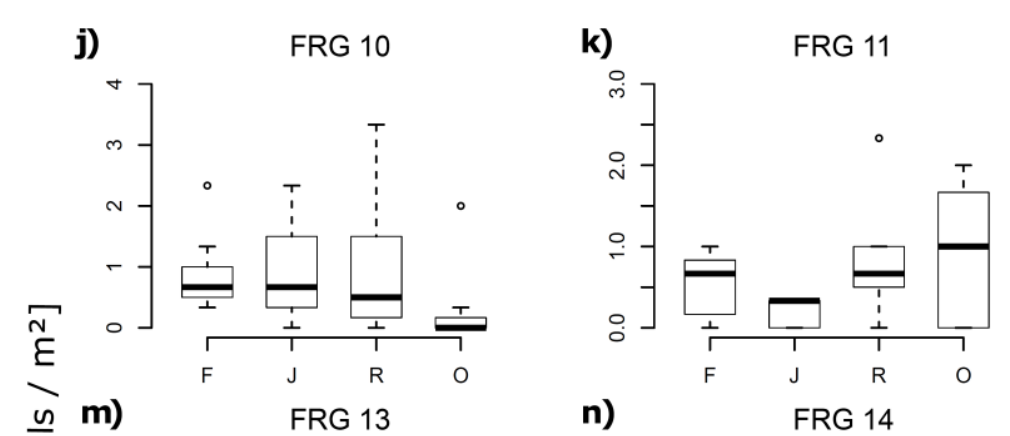

n)
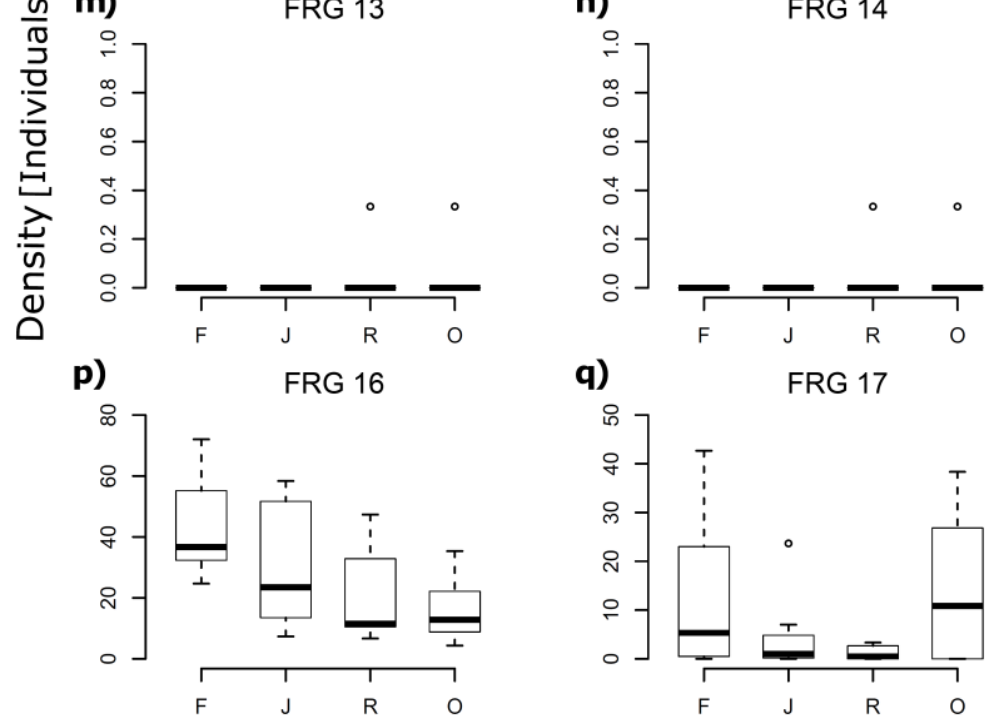

I)

FRG 12

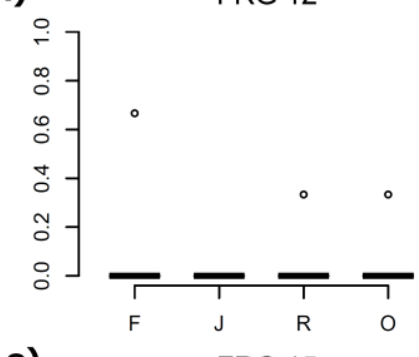

o)

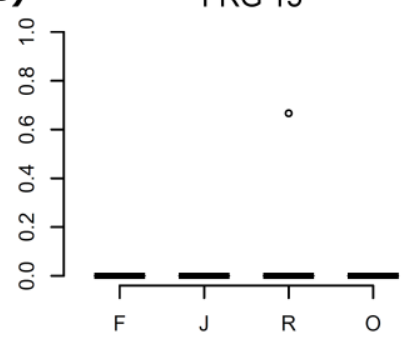

q)

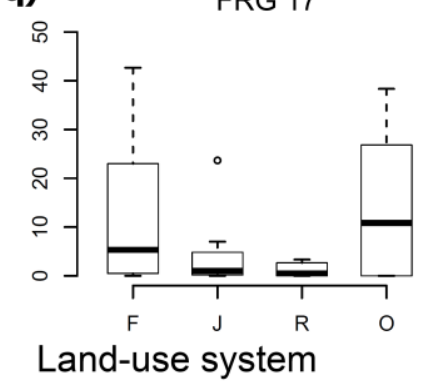

r)

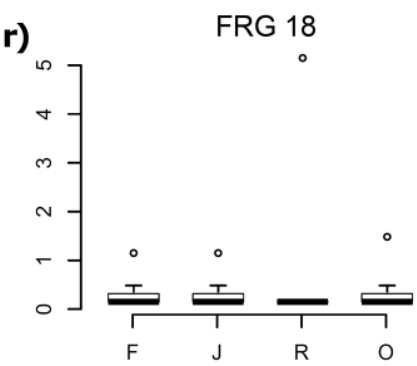




\section{SUPPLEMENTARY INFORMATION - CHAPTER 5}

Supplementary Figure 5.1 Study regions in a) Indonesia and b) Germany. The left-hand panels show the island of Sumatra within Indonesia and the country of Germany within Western Europe, with a magnification of the study regions in the right-hand panels. Green and yellow circles in a) denote the sampling plot locations for Bukit Duabelas and Harapan landscapes, respectively. Red, blue and orange circles in b) denote the sampling plot locations in the Swabian Alb Biosphere Reserve, Hainich National Park and the Schorfheide-Chorin Biosphere Reserve, respectively.

a) Indonesia

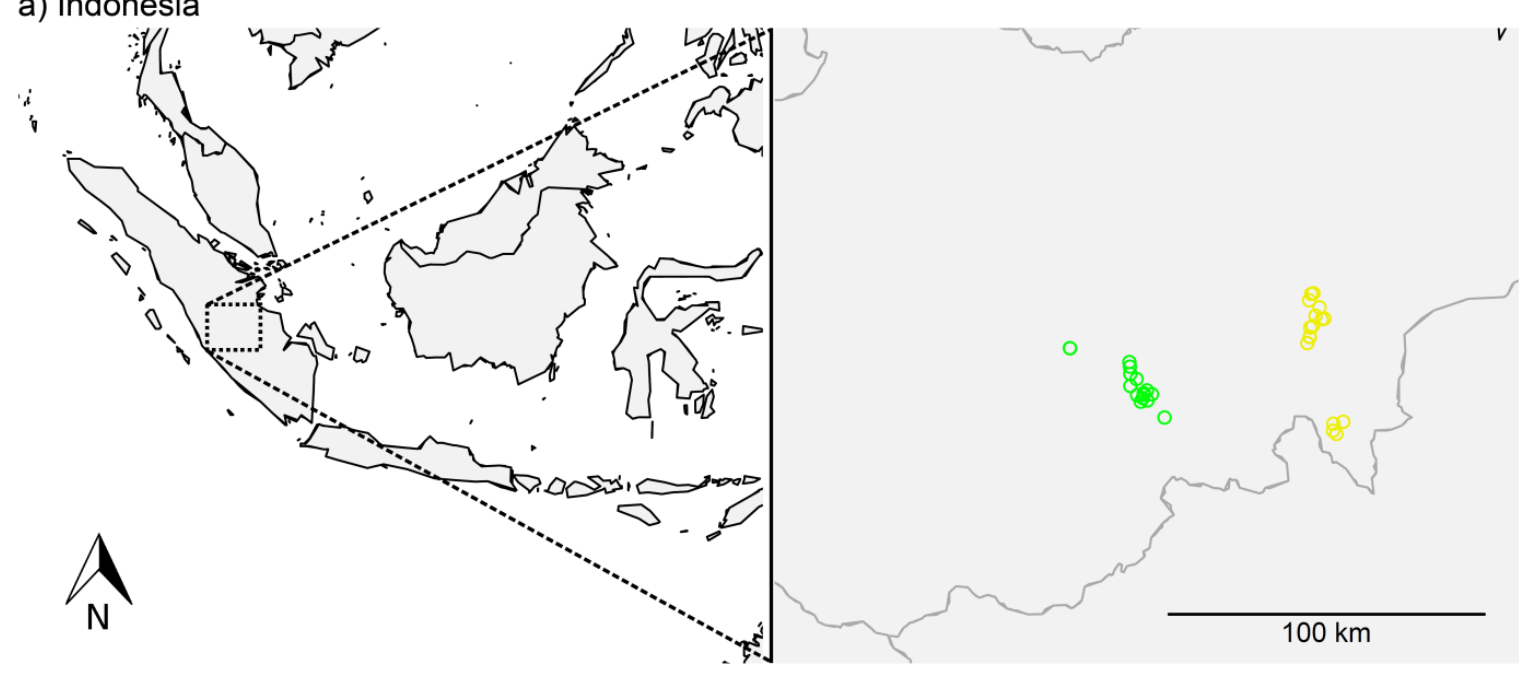

b) Germany

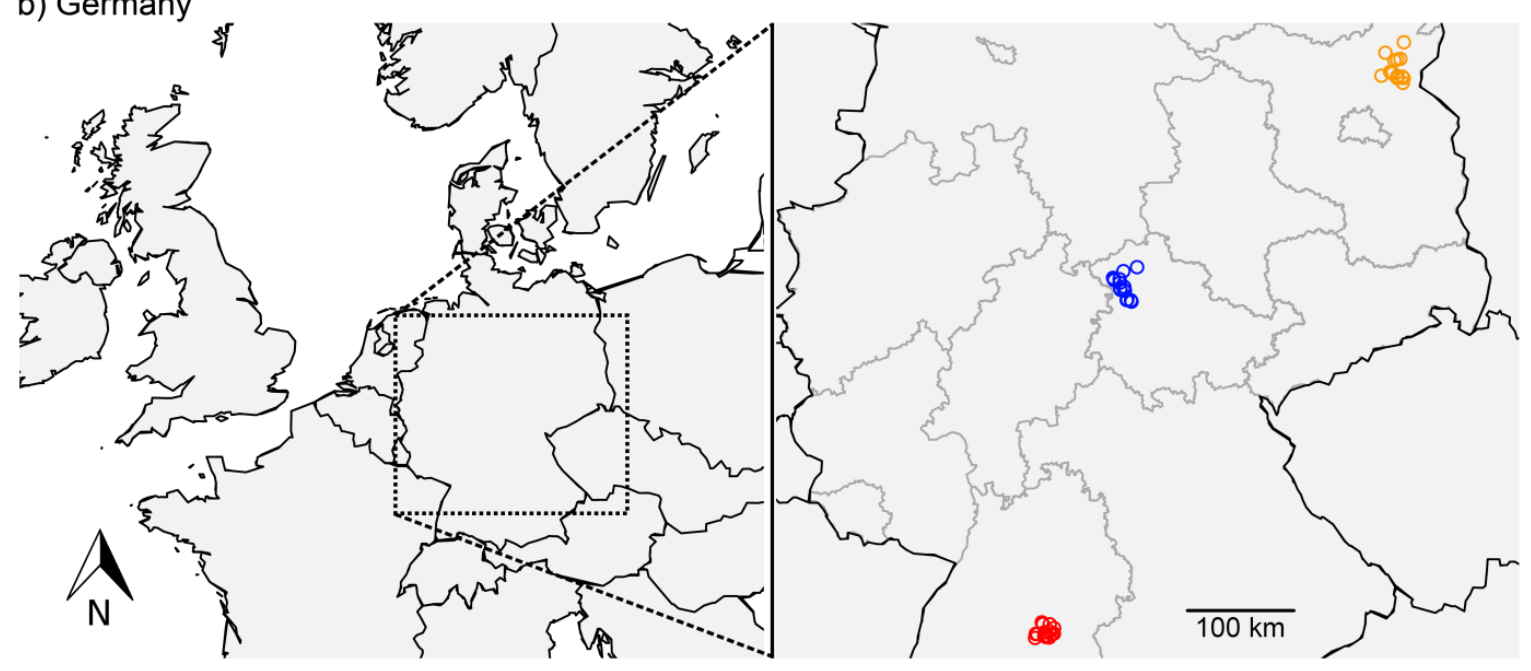


Supplementary Table 5.1 Variables used to construct environmental dissimilarity matrices for the 'environmental distance' variable in the path model. $\mathrm{R}^{2}$ and $\mathrm{p}$ values are from the permutational vector fitting on NMDS ordinations of litter macroinvertebrate communities. Both untransformed and logged variables are shown, giving a total of 30 variables. Variables that were selected to be included in the environmental dissimilarity matrices are indicated by bold-face parameter values for the Indonesian and German data sets.

\begin{tabular}{|c|c|c|c|c|}
\hline \multirow{2}{*}{ Factor } & \multicolumn{2}{|c|}{ Indonesia } & \multicolumn{2}{|c|}{ Germany } \\
\hline & $\mathbf{R}^{2}$ & p-value & $\mathbf{R}^{2}$ & p-value \\
\hline litter depth & 0.540 & 0.000 & 0.017 & 0.673 \\
\hline soil pH & 0.307 & 0.005 & 0.456 & 0.000 \\
\hline $\mathrm{C}(\mathrm{mg})$ & 0.179 & 0.055 & 0.032 & 0.481 \\
\hline $\mathrm{N}(\mathrm{mg})$ & 0.064 & 0.379 & 0.243 & 0.002 \\
\hline $\mathrm{P}(\mathrm{mg})$ & 0.201 & 0.036 & 0.098 & 0.102 \\
\hline $\mathrm{Al}(\mathrm{mg})$ & 0.151 & 0.093 & 0.424 & 0.000 \\
\hline $\mathrm{Ca}(\mathrm{mg})$ & 0.161 & 0.077 & 0.517 & 0.000 \\
\hline $\mathrm{Fe}(\mathrm{mg})$ & 0.135 & 0.115 & 0.270 & 0.001 \\
\hline $\mathrm{K}(\mathrm{mg})$ & 0.245 & 0.016 & 0.286 & 0.001 \\
\hline $\operatorname{Mg}(\mathrm{mg})$ & 0.001 & 0.992 & 0.158 & 0.020 \\
\hline Mn (mg) & 0.169 & 0.065 & 0.465 & 0.000 \\
\hline $\mathrm{Na}(\mathrm{mg})$ & 0.011 & 0.843 & 0.190 & 0.010 \\
\hline $\mathrm{S}(\mathrm{mg})$ & 0.169 & 0.068 & 0.064 & 0.220 \\
\hline mean soil moisture & 0.207 & 0.033 & 0.744 & 0.000 \\
\hline mean soil temperature & 0.432 & 0.000 & 0.385 & 0.000 \\
\hline log litter depth & 0.476 & 0.000 & 0.028 & 0.528 \\
\hline $\log$ soil $\mathrm{pH}$ & 0.323 & 0.003 & 0.418 & 0.000 \\
\hline $\log C(\mathrm{mg})$ & 0.061 & 0.410 & 0.008 & 0.840 \\
\hline $\log N(\mathrm{mg})$ & 0.107 & 0.191 & 0.262 & 0.001 \\
\hline $\log \mathrm{P}(\mathrm{mg})$ & 0.216 & 0.028 & 0.108 & 0.079 \\
\hline $\log \mathrm{Al}(\mathrm{mg})$ & 0.157 & 0.088 & 0.594 & 0.000 \\
\hline $\log \mathrm{Ca}(\mathrm{mg})$ & 0.230 & 0.024 & 0.422 & 0.000 \\
\hline $\log \mathrm{Fe}(\mathrm{mg})$ & 0.174 & 0.061 & 0.276 & 0.001 \\
\hline $\log K(\mathrm{mg})$ & 0.361 & 0.001 & 0.328 & 0.000 \\
\hline $\log \mathrm{Mg}(\mathrm{mg})$ & 0.007 & 0.906 & 0.341 & 0.000 \\
\hline $\log \mathrm{Mn}(\mathrm{mg})$ & 0.181 & 0.052 & 0.466 & 0.000 \\
\hline $\log \mathrm{Na}(\mathrm{mg})$ & 0.010 & 0.868 & 0.191 & 0.009 \\
\hline $\log S(m g)$ & 0.177 & 0.059 & 0.114 & 0.061 \\
\hline log mean soil moisture & 0.113 & 0.173 & 0.766 & 0.000 \\
\hline log mean soil temperature & 0.437 & 0.000 & 0.286 & 0.000 \\
\hline
\end{tabular}


Supplementary Table 5.2 Parameters and their estimates from each of the two path models (from the Indonesian and German data sets). All response and predictor variables are dissimilarity matrices (e.g., 'biomass' denotes dissimilarity among plots in total biomass values).

\begin{tabular}{|c|c|c|c|c|c|}
\hline Response & & Predictor & $\begin{array}{l}\text { Unstandardised } \\
\text { coefficients }\end{array}$ & $\begin{array}{c}\text { Range- } \\
\text { standardized } \\
\text { coefficients }\end{array}$ & $\mathbf{P}$ \\
\hline \multicolumn{6}{|l|}{ Indonesia } \\
\hline $\begin{array}{l}\text { environmental } \\
\text { dist. }\end{array}$ & $<-$ & geographic dist. & 0.003 & 0.096 & 0.008 \\
\hline$\beta$-diversity & $<--$ & geographic dist. & 0.000 & 0.108 & $<0.001$ \\
\hline$\beta$-diversity & $<--$ & environmental dist. & 0.020 & 0.225 & $<0.001$ \\
\hline$\alpha$-diversity & $<--$ & $\beta$-diversity & 3.709 & 0.592 & $<0.001$ \\
\hline FDis & $<--$ & geographic dist. & 0.000 & 0.096 & 0.010 \\
\hline biomass & $<--$ & $\alpha$-diversity & 0.797 & 0.409 & $<0.001$ \\
\hline energy flux & $<--$ & $\alpha$-diversity & 0.432 & 0.269 & $<0.001$ \\
\hline energy flux & $<--$ & biomass & 0.603 & 0.733 & $<0.001$ \\
\hline $\begin{array}{l}\text { Germany } \\
\text { environmental } \\
\text { dist. }\end{array}$ & $<-$ & geographic dist. & 0.001 & 0.195 & $<0.001$ \\
\hline$\beta$-diversity & $<--$ & geographic dist. & 0.000 & 0.227 & $<0.001$ \\
\hline$\beta$-diversity & $<--$ & environmental dist. & 0.019 & 0.157 & 0.020 \\
\hline$\alpha$-diversity & $<--$ & geographic dist. & -0.000 & -0.094 & 0.006 \\
\hline$\alpha$-diversity & $<--$ & $\beta$-diversity & 1.020 & 0.411 & $<0.001$ \\
\hline biomass & $<-$ & geographic dist. & 0.000 & 0.057 & 0.020 \\
\hline biomass & $<--$ & $\alpha$-diversity & 1.374 & 0.351 & $<0.001$ \\
\hline biomass & $<--$ & $\beta$-diversity & 3.364 & 0.347 & $<0.001$ \\
\hline energy flux & $<--$ & geographic dist. & -0.000 & -0.054 & 0.001 \\
\hline energy flux & $<--$ & $\alpha$-diversity & 0.579 & 0.208 & $<0.001$ \\
\hline energy flux & $<--$ & biomass & 0.543 & 0.765 & $<0.001$ \\
\hline
\end{tabular}




\section{ACKNOWLEDGEMENTS}

I would firstly like to thank my supervisor, Ulrich Brose, for making this $\mathrm{PhD}$ project possible. He has always encouraged me to think outside the square and to be creative, somehow always managed to provide support when it was needed, and even made sure that there were plenty of fun times throughout! I have developed academically more than I could have imagined because of his excellent supervision and I think I could not have found a more perfect working group at this stage in my career.

I would also like to thank my second supervisor, Stefan Scheu. Although I did not end up working with him closely, he has steered the Titanic CRC990 through iceberg-ridden waters and managed to keep sailing on ahead, thus making my $\mathrm{PhD}$ possible. I must also mention that a ship captain requires a good crew to sail successfully and, therefore, I am also highly grateful too all of the CRC990 administrative staff, in both Germany and Indonesia, for somehow bringing this enormous project together and making it work. Thinking back to 2012, it is truly incredible that the project has made it to what it is today, thanks to the extremely hard work of all the staff! Also, special thanks go to our super amazing team of research assistants in Indonesia: Megawati, Rizky Nazarreta, Keisha Disa Putirama, and Rosario Reza Valentino Lasse.

I especially thank my PhD wife/husband (that's still undecided?), Malte Jochum. I seriously don't know how I would have made it through all of this without you. Together, we've braved German and Indonesian bureaucracy, giant spiders and wasps, risk of sweating to death (well, you anyway), hordes of mosquitos, and months of 7's on the Bristol scale. Working with you has been a blast and I am incredibly grateful to have had you as a scientific collaborator and a friend! This leads me to all of the people I have to thank who have been through this IndonesianGerman adventure with me: Steffen Mumme, for being an A-class, first master's student to supervise; you kept me going with plenty of pool games and Heinekens, and I am seriously impressed that you hung in there through some extreme typhoid and back agony and managed to pull out the other side with a great master's thesis and publication! Mimi Teuscher, you've been a real colleague (and a great friend as well) - shall we remember the times that we were happy because it depends on the amount. There are really too many people to thank here, and I'm not going to name everyone because I'll surely leave important people out, but thanks to the Americans (you know who you are), the Bali crew (including Jamie Fox), the pool player's crew (that just about includes everyone I guess), oh, and of course the Bogor language course crew (remember: disini senang, disana senang). You guys have all been a real pleasure to work with and I'm really grateful to have met you all!

Also, I want to thank the current and past EcoNetLab gang that I've had the opportunity to work with: Björn, David, Flo, Patrick, Christoph, Lena, Tony, Ka, Amrei, Gregor, Birgit, Christian, Esra, Dianna Toby, and Lucas, for many great discussions, laughs, fun times and for 
generally making my time in Göttingen an enjoyable experience. Of course, special thanks to Rita Füllgrabe-Schmidt for helping me to organise my professional (and often personal) life. To all my friends that I have not mentioned so far, both in Göttingen and around the globe, thanks for lightening the load by providing good times that involve non-PhD-related activities and reminding me that life outside my $\mathrm{PhD}$ does exist.

It should go without saying that I am extremely grateful to my parents. I don't need to have a $\mathrm{PhD}$ in biology to know that this $\mathrm{PhD}$ thesis would literally be impossible without them. Thank you for raising me to always think critically, question generally accepted views, never rule out the improbable, think creatively and open-mindedly, and also always fostering the idea that I am capable of doing whatever I set my mind to (although, as a scientist, I now realise that unaided flight is out of the question). Additionally, I want thank my erzgebirgische family that has given me much-appreciated and needed family support so far from my homeland(s). "HEL", und vielen dank für alles! Ich bin sehr fröhlich dass habe ich euch kennengelernt!

Finally, and most importantly, I want to thank my amazing girlfriend/fiancé-beyonce for supporting me throughout the majority of my PhD. I know that it's been tough competing with my $\mathrm{PhD}$ wife/husband, putting up with my incessant blabbing about my thesis, dealing with my emotional madness, living with someone who often forgets to change out of his schlafanzug the whole day because he's too busy with his thesis, and won't even remember to fix that bloody water rooster. You've been unbelievably supportive in every way and managed to keep me laughing the whole time! Parts of this passage might look oddly familiar to you (hust hust), but I am inexpressibly grateful to have had you by my side through the last couple years, I love you and I am incredibly lucky to be the one to say that I'm looking forward to our future together! 